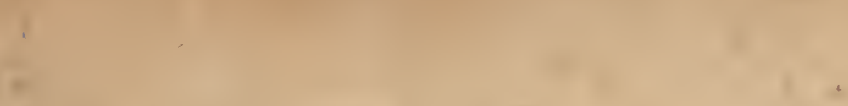

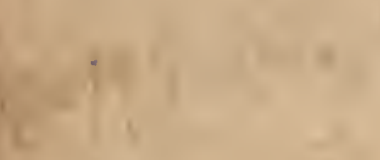

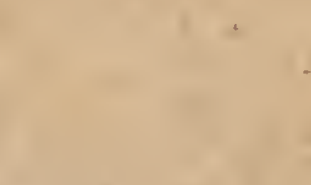<smiles>C1CCCCCCCCCCC1</smiles>
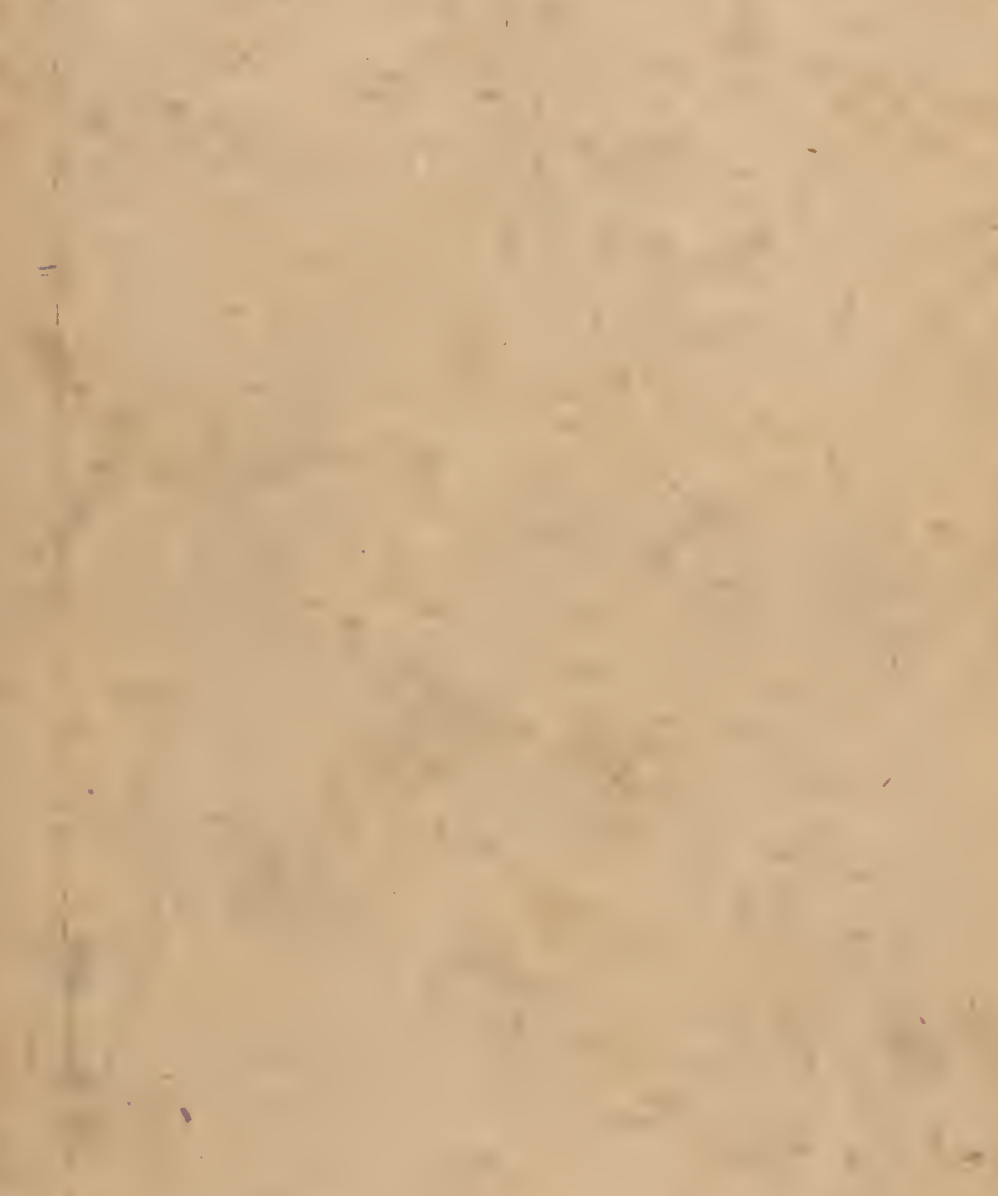

4

-

-

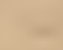

$-$

.

(n)

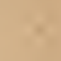

1 





\title{
EPITOME ENTOMOLOGIAE
}

\author{
F A B R I C I A N A E
}

S I V E

\section{NOMENCLATOR}

ENTOMOLOGICVS EMENDATVS

\author{
S ISTENS
}

FABRICIANI SYSTEMATIS CVM LINNEANO COMPARATIONEM

$$
\text { A D I E C T I S }
$$

CHARACTERIBVS ORDINVM ET GENERVM, SPECIEBVS NOVIS ALIORVM ENTOMOLOGORVM, INSECTORVM HABITATIONIBVS NOMINIBVS GERMANORVM FRANCOGALLORVM. ANGI.ORVM.

CVM INDICIBVS ET BIBLIOTHECA FABRICIANA.

$$
L I P \text { I } I \text { I A E }
$$

A U D I O. GOTTLOB,FEIND

$$
\text { 1 } 7.9 \%
$$


O 


\section{LECTORI, ER VDITO \\ S. D. \\ E D I T O R.}

Sunt absque dubio infignia in difficili entomológico ftudio Io. Chrilliani Fabricii merita, quibus a viginti duobus retro annis haec hiftoriae, naturalis pars egregie emendata atque locupletata fuit, quamuis hic rerum entomologicarum praeclariffimi viri labor a paucis tantum excultus atque laudatus fuerit. Forfan Fabricius huic fuo ftudio obftacula pofuit ipfe et quidem eo, quod terminorum technicorum fenfum non adcurate fatis atque dilucide explicauerit, numerum generum nimis auxerit - cum e contrario Linneus nimis parcus fuerit in conftituendis generibus - a valdopere obfcuro et occulto charactere in minutis inprimis infectis partitiones defumferit, differentiam fpecificam non fatis diligenter in fpecierum defcriptionibus faepenumero indicauerit neque optima atque perfpicua demonftrandi compendia, quemadmodum fere lemper Linneus fecit, elegerit. At enim vero hoc verum, imo verifimum elt, Fabricianum entomologicum ftudium in ampliffima corporum naturalium claffe prae caeteris eminere atque omnino dignum effe, quod ab omnis aeui hiftoriae naturalis cultoribus folidius cognofcatur. 
Quapropter compofuimus ex noua entomologia fyltematica aucta atque emendata a. I792 - 1794 edita hancce epitomen, ut huius egregiae atque praeftantis eruditionis inter entomologos facilior fieret communicatio, Quae haec noftra epitome contineat, in infcriptione ipfus fatim indicauimus. Caeterum in votis erat, criticae entomologiae fpecimina quaedam adjicere; reuocarunt autem $\mathrm{ab}$ hoc inflituto nos inprimis plerorumque entomologorum hodiernorum in hac methodologiae parte incuria atque defidia. Fabricianis Agonatis Daldorfiana genera fubjunximus atque praeterea fequentibus fignis inprimis uf fumus
C. Cramer.
O. Olivier.
Deg. Degeer.
P. Pallas.
B. Esper.
S. N. Speries nova.
H. Herbft. W. Wiener Verzeich.
M. Mantifja infector. niis.
Fabr.
P. Panzer.

Reliqua explicationem non defiderant.

Scr. XIV. Calend. Iunii cIoIoccxcvır. 


\section{IO. CHRISTI. FABRICII}

\section{BIBLIOTHECA ENTOMOLOGICA.}

I. Syllema entomologiae. Flensb. 1 775.8 .

2. Genera Infectorum. Kilon. I 776.8 .

3. Philofophia entomologica, Hamburgi 1778.8 .

4. Species Infectorum. 2. Vol, Hainburgi I $78 x, 8$.

5. Mantifa Infectorum. Hafuiae 1787. 2 Vol. 8.

6. Entomologia fyfematica emendata et aucfa. Hafniac. Tom. I-IV. I $792-94$. 4. Voll. 8 .

7. Nomenclator entomologicus fecundurn entomologiam fyftematicam ill. Fabricii. - confcriptus a Friderico Webero. Chilonii et Hamburgi, I 795.8 .

8. Index alphabeticus in I. C. Fabricii Entomologinm fyftematicam' emendatam et auctam, ordines, genera et fpecies continens. Hafniac 1796.8 . 
x. Von der weifen Ameife in den Schriften der Ge. Selifchuft naturf. Fraunde in Berlin.

2. Betrachtung über die Syfteme der Entomologie; ebendaf.

3. Dé organo auditus infectorum in Actis Soc. Scient. Hafn.

4. Nova Infectorum genera in Actis Soc. Naturae Scrutat. Hafn.

5. De Philaena faccharali et Noctua Gofypii in iisdem Açis.

6. Nova infectorum genera in Actis Suc. Naturae Scru. tatorum Parifienfis. 


\title{
C. O M P A R A T I O
}

SYSTEMATIS ENTOMOLOGICI LINNEANI CVM FABRICIANO.

\author{
O R D O I. \\ COLEOPTERA. (Eleuterata F.) *) \\ * Antennis clavatis extrorfum incraffatis.
}

I. Scarauaevs. 2.

Trox 7 .

Trichius 75 .

Cetonia 76.

Melolontha 77.

Lethrus I.

2. LVCANVS 80 .

Paffalus 81 .

Melyris $3 \mathrm{I}$.

3. Dermestes 32.

Sphaeridium 5.

Nitidula 40.

Synodendron 94**)

*) E. IuI. Elentcratum. V1. Vlonatum. S. Syuiftatum, P. Piezatum. O. Olonatum. M. Mitofatum. Vn. Vinogatum. Ag. Agonatum, G. Gloffatum. R. Ryngo. tuill. Au. Antliatum.

*) Nouren iuiquum facile cum nominibus Botanicorum confundendun, quemadnoduus hodierni rerum naturalium deferiptores poft Linneuni faepenumero in denomiuandis corporibus naturalibus valdopere peccarunt. 
Apate 95.

Boftrichus. 96 .

Colydium 104.

Mycetophagus 105. Hypophlacus 106. Hexodon 3.

4. Hister 4.

5. BYRRHVS 6 ,

Anthrenus 42.

6. Gyrinvs 25.

Erodius 9.

7. Attelabys ioo.

Clerus 27.

Notoxus 28.

Spondylis y3.

Vpis I 12.

8. Cverculio ioz.

Anthribus 98.

Brachycerus 99.

Rbinomacer IOI.

Brentus 103.

9. Silpha 39.

Sphaeridium 5.

Opatrum 8.

Elophorus 26.

Nicrophorus 38 .

Nitidula 40.

Scaphidium I I 0.

Ips III.

Tetratoma $10 \mathrm{~g}$.

Heterocernis $4 \mathrm{I}$.

IO. CocCinelia 43.

Sphacridium 5.

Erotylus 48 . 
* Antennis filiformibuss.

I I. BRvCHVs 97 .

Codypius 67.

12. Cassida 44 .

Blatta Vl. I I 9 .

Scaurves 10.

Sepidium I2.

13. Ptinvs 34.

Ariobium 33 .

Ptilinus 35.

I 4. Chrysomela 45 .

Helops 17 .

Crioceris 46.

Galleruca 47.

Erotylus 48.

Ciftela 50.

Cryprocephalus 56 .

Tillus 59.

l.agria 60.

Tritoma 108.

Diaperis 113 .

Dryops 58.

I 5. HISPA 57.

Cebris 49.

Zygia $5 \mathrm{I}$.

Zonitis 52.

I6. MELOE II 4 .

Notoxus 27.

Malachius 30.

Apálús 53 .

Alurnus 54.

Sagra 55.

Cerocoma $6 \mathrm{r}$.

Lytta 62.

Mylabris 63. 
17. TenerRio I5.

Scarites II.

Pimelia 13.

Blaps I 4.

Tragofita 16 .

Helops 17.

Lyctus 107.

Cychrus.

I8. LAMPYRIS 68 .

Pyrochroa 70 .

Omalysus 69 .

Lycus 7 I.

I9. Mordella 73.

Ripiphorus 72.

20. Staphylinvs II5.

Oxyporus II 6 .

Paederus I I 7 .

4*: Antennis Setaceis.

21. Cerambyx 83.

Prionus 82.

Lamia 84.

Stenocorus 85 .

Calopus 86.

Rhagium 87.

Saperda 88.

Callidium 89.

22. Leitura 90.

Donacia 74.

23. Cantharis 29.

Horia 64 .

Malachius 30 .

Lymexylon 65.

Cucujus 66. 
Necydalis 91.

24. Elater 79.

Melafis 36.

Parnus 37.

25. Cicindela 20.

Elaphrus 21.

26. JVPRESTIS 78 .

Manticora I8.

27. Dytiscvs 24.

Hydrophilus 23.

2S. Carabvs 19.

Scolytus 22.

29. Necydalis $9 I$.

Moiorchus 92 .

30. Fonficvia VI. I 18.

O R D O II.

HEMIPTERA. (Vlonata et Rliyngota F.)

1. BLatta VL. IIg.

2. MANTIS Vl. I 20.

3. GRYLLVS V!. I25.

Acrydium VI. I 2 I.

Truxalis VI. I 22.

Acheta VI. 123.

Locufta Vl. I 24.

4. Fvlgora R. 201.

5. Cicada R. 204 .

Membracis R. 202.

Tettigonia R. 203.

Cercopis R. 205.

6. Notonecta R. 206.

Sigara R. 207. 
7. Nepa R. 208.

Ranatra R. 209.

Naucoris R. 2 ro.

8. Cimex R. 2 I2.

Acanthia R. 2 II.

Coreus R. 2 I 3.

Lygaeus R. 214.

Miris R. 215.

Gerris R. 2 I 6.

Reduvius R. 217.

9. APHIS K. 219.

IO. Chermes R. 220.

II. Coccus R. 22 I.

I2. ThR.IPS R. 222.

\section{O R D O III.}

LEPIDOPTERA. (Gloflata Fabricii.)

I. Papilio G. 186.

Hesperia G. 187.

2. SpHinx G. 188.

Sefia G. 189.

Zygaena G. 190.

3. Phalaena G. I.96.

Bombyx G. I 91 .

Coffus G. 192 .

Hepialus G. 193.

Noćtua G. 194 .

Hyblaca G. 195. .

Pyralis G. 197 .

Tinea G. 198.

Alucita G. I 99.

Pterophorus G. 200. 
O R D O IV.

NEVROPTERA. (Odonata et Syniftata Fabr.)

I. Libelluta O. 164 ,

Aeshna 0. 165 . $^{\circ}$

Agrion $0 . \pm 66$.

2. EPHEMERA S. I 28 .

3. MYRMELEON S. 133.

Afcalaphus S. I 34.

4. Phryganea S. 130.

Semblis S. 129 .

5. Hemerobivs S. I3I.

Semhlis S. 129 .

Termes S. 132 .

6. Panorpa S. I35."

7. Raphidia S. 136 .

Mantis S. 120.

Hemerobius S. I $\mathbf{3}$ I.

O R D O V.

HYMENOPTEKA. (Piezata Fabs:)

I. Cynips P. 137.

Iclineumon $\dot{\mathrm{P}} .140$.

2. Tenthredo P. I 38 .

3. SIREX P. I 39.

4. ICHNEVMON P. I 40.

5. SPHEX P. I 43.

Euania P. I 4 I.

Chalcis P. I 42.

Mellinus P. I 53 . 
Philantus P, I54, Crabro P. 155 .

6. Chrysis P. 147 .

Larra P. 144.

Tiphia P. 145.

4 Scolia P. 146.

Thymus P. 148.

Leucopfis P. 149 .

7. VesPa. P. I $5 x$.

Chalcis P. 142. Bembex P. 150. Malaris P. I 52. Mellimus P. 153 . Crabro P. 155.

8. APIS P. 158.

Sphex P. 143. licmbex P. 150. Hylaeus P. 156. Andraena P. 157. Eucera P. 159. Nomada P. I60.

9. Formica P. I6I.

1o. Mvtilla P. I63.

Ichueumon P. I 40.

Dorylus P. I62.

$$
\text { OR D O VI. }
$$

DIPTERA. (Antliata Fabr.)

I. Oestavs 223.

2. Tipvla. 224.

3. Mvsca 233.

Mydas 225.

Bibio 226 . 
Anthrax 227.

Stratiomys 228,

Nemotelus 229.

Rhagio 230.

Syrphus 232.

\section{Ceria 23 I.}

4. Tabanvs 234.

5. CVLex 240.

6. EMPIS $24 \mathrm{r}$.

7. Conors 237.

Rhingia 235.

Stomoxys $23 \%$.

Myopa 239.

8. Asilvs 236 .

9. Bombylivs 24,2 .

Voluccella 243.

Cytherea 244 .

10. Hippobosca 245.

O R D O VII.

$\triangle \mathrm{I} \quad \mathrm{T}$ E $\mathrm{R}$ A.

* Pedibus fex; capite a thorace difcreto.

I. Lepisma S. 126.

2. Podvra S. 127.

3. Termes S. 232.

Hemerobius S. 13 I.

4. Pediculvs An. 248.

5. Pvlex R, 218.

* * Pedibus 8 - I4; capite thoraceque znnitis.

6. Acarus An. 249.

Trombidium $\mathrm{V} \mathbf{n}, \mathbf{7 0}$. 
7. Phalanglvm Vir. I72.

Tarantula Vn. I 73.

Scorpio Vt. I 74 .

Pycriogonum An. 246.

Nymplion An. 247.

8. ARANEA Vn. I 7 I.

9. SCORPIO. Vn. I 74.

Io. CANCER. Ag. I 75 .

Pagurus I 76 .

Galathea 177 .

Hippa 178.

Scyllarus 179 .

Aftacus 180 .

Squilla 184.

Gammarus 185 .

II. Monocrevs Ag. I 82.

Limulus Ag. I 8 I.

12. Oniscus M. 169 .

Cymothoa Ag. I 83 .

*** Pedibus plusibus, capite a thorace difcreto.

13. SCOLOPENDRA M. I67.

I4. IVLVS M. I 68 . 


\section{EPITOME ENTOMOLOGIAEFABRICIANAE.}

\section{INSECTORVM CLASSES}

\section{SEC. I O. CHRISTI. FABR I I V M.}

I. Elevterata maxilla nuda libera.

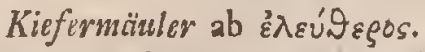

2. Vlonata palpi quatuor, maxilla tecta galea obtufa. Heimkiefer at oũ $\lambda$ ov.

3. Sxnistata palpi quatuor, maxilla connata cum labio.

Kieferlippen a ouvisnp..

4. Prezati palpi quatuor, maxilla cornea, comprefla, faepe elongata.

Hormuinuler a $\pi$ lí $3 \omega$.

5. Odonata palpi duo breues, maxilla cornea dentata. Zahninü̈uler ab ód's.

6. Mrtosata palpi duo, tmaxilla filiformis membranacè̈:

Fadenmizinler a $\mu$ ítos.

7. Vnogata palpi duo porrecti, maxilla cornea uhiguiculata.

Haakenmäüler ab "ैvv

8. LGonata. palpi faepius fex, maxilla omuino nulla. Kinnlofe ab áyóvatos.

9. Grossat a lingua firalis inter palpos reflexos, fupofos. Zungermäzuler a $\gamma \lambda \tilde{\omega} \sigma \sigma \alpha$.

I0. RYNGOTA os roftro: vagina articulata:

Riilfeimäuler' ab éúryos.

II. Antriata os probofcide atque haufello،

SchüpfriüJel ab átr $\lambda \dot{o}$ oy. 


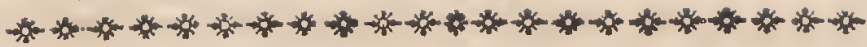

\section{CHARACTERES ORDINVM ET GENERVM.}

CLASSIS I. ELEVTERATA.

* Antennis roftro elongato corneo infidentibus.

102. Cvrcviro. Maxilla unidentata. Antennae clava folida: articulo fecundo longifinno.

103. BRENTVS. Roftum rectum, porrectum, cylindricum. Antennae moniliformes ultra medium infertae.

100. Aтtelabvs. Rofrum elongatum, incurvum. Maxilla bifida. Antennac extrorfun crallines.

IOI. RHINOMACES. Palpi quatuor extrorfum crafiores: articulo ultimo obliquo, truncato: Antenñae fetaceae.

98. ANтнRibvs. Palpi quatuor filiformes. Maxilla bifda. Labium bifidum. Antennae articulis rotundatis: ultimis tribus ovatis craflioribus; ultimo acuto.

99. Brachycervs. Paipi quatuor breviffimi. Labium corneum, rotundatum. Antenuae extrorfum craffrores.

* Antennis clava lamellata.

80. Lvcunvs. Penicilli duo fub labio palpigeri.

8I. Passälvs. Palpi filiformes. Maxilla cornea, ar. cuata, dentata, acuta. Labium finuatum.

2. Scarabaevs. Palpi filiformes. Labium emarginatum: apicibus elongatis, palpigeris.

7. Trox. Palpi quatuor capitati. Maxilla bifida. Labium corneum, emarginatum.

4. Hexodon. Palpi antici fubclavati. Maxilla cornea tridentata: dentibus fiflis. Labium late emarginatum.

77. Melolontha. Maxilla brevis, cornea, apice nultidentata.

75. Trichivs. Palpi quatuor filiformes, Maxilla bifida. 76. 
76. Cetonia. Maxilla apice fetofa. Labium coriaceum, emarginatum, palpos tegens.

94. SYNODENDRON. Palpi filiformes. Labium filiforme, corneum, apice palpigerum.

1. Lethrvs. Maudibula adunco falcata. Antennac cla-

va tuncata, tunicata.

*** Antennis clava perfoliata.

95. AтAте. Palpi aequales, filiformes. Maxilla unidentata, Labium membranaceum, truncatum.

32. Melyris. Palpi aequales, filiformes. Maxilla unidentata, acuta. Labium clavatum, emarginatum.

III. Irs. Palpi aequalcs, filiformes: atticulo ultimo obtufo, truncato. Maxilla bifida. Labium trunca. tum, einarginatum.

6. Brrrivs, Palpi aequales: articulo ultimo obtufo, truncato, fubcraftiori. Labium porrectum, bifidum: laciniis conniventibus.

104. Colydivm, Palpi aequales, clavati. Labium membranaceum, bifidum.

32. Dermestes. Palpi inaequales, filiformes. Maxilla bifida. Labium corneum, obtufum.

110. Scaphidrvm. Palpi inaequales, filiformes: articulo ultimo acuto. Maxilla membranacea, bifida. Lia bium corneum, rotundatum.

5. Sphafridvm. Palpi inaequales, filiformes. Labium - quadratum, emarginatum.

109. Tetratoma. Palpi inaequales, craffufculi. Maxilla membranacea, bifida. Labium breve, rotundatum, integrum.

67. Cossyphys. Palpi inaequales: anteriores fecuriformes. Maxilla bifida. Labium integrum.

x08. Tritoma. Palpi antici fecuriformes. Labium enarginatum.

38. Nicrophorys. Labium cordato emarginatum, erenatum. 
39. Sílpha. Maxilla unidentata. Lahium dilatatum, bifidum.

24. Hydrophilvs. Palpi filiformes. Maxilla bifida. Labinn corneum, fubenarginatum.

I12. Diaperts. Palpi fubfiliformes. Maxilla bifida. l.abium cylindricum apice palpigerum.

**** Antennis clava folida.

3. Hister. Maxilla unidentata. Lahium corneum cy. lindricum, integrum.

96. Bostrichys. Maxilla cornea. Labium cylindricum apice palpigerum.

107. Lyctus. Palpi breviflini, filiformes. Maxilla menubranacea, bifida,

42. Anthrenvs. Palpi inaequales, filiformes. Maxilla linearis, bifida. Labium integrum.

26. Elophorvs. Maxilla cornea, apice membranacea. Labium corneum, quadratum, apice palpigerum.

40. Nitrovea. Palpi filiformes. Maxilla cylindrica, membrauacea. Labium cylindricum, integrum, medio palpigeruns.

43. Cuccinelza, Palpi antici fecuriformes, poftici filiformes. Iabium cylindricum.

- * Anternis flabelliformibus.

106. Hypophlaevs. Palpi aequales, clavati. Maxilla membranacea, únidentata. Labium filiforme medio palpigerum.

35. Pricinvs. Paipi fubaequales, filiformes. Maxilla bifida. Labium breve, fubemarginatum.

36. Melasis. Palpi clavati: articulo ultimo ovato. Labium membranaceun, integrum.

72. Rhipquorvs. Palpi inaequales, filiformes. Máxil. la breviffina, ovata. Labium acutum. * *2* * Antennis extrorfum crafioribus.

16. Trogosita. Palpi aequales: articulo ultimo obtufo, truncato. Maxilla unidentata. Labium corneum, bifidum: laciniis rotundatis, ciliatis. 
105. Mycetophagvs, Palpi inaequales: articulo ultimo obtufo. Maxilla unidentata. Labium rotundatum, integrum.

15. Tenebrio. Palpi inaequales: antici fubclavati, poftici filiformes. Maxilla bifita. Labium corneum, integrum.

63. Mylabris. Palpi inaequales, filiformes. Maxilla connea, apice bifida. Labium fubernarginatum.

27. Clervs. l'alpi inaequales: pollici longiores, fecuriformes.

28. Noтоxvis. Palpi inaequales, fecuriformes. Maxilla usidentata. Labiun bifidum: laciuiis conniventibus, obrufis.

33. Аловим. Palpi aequales, clavati. Maxilla cornea. Labium integrum.

48. Erotylus. Palpi inaequales: antici fecuriformes, poftici clavati, Labium corneum, apice diltataum, emarginatum.

*末*** Antennis moniliformibus.

9. Erodivs. Palpi aequales, filiformes. Maxilla cornea, bifida. Labium corneum, emarginatum.

61. Cerocoma. Palpi aequales, filiformes. Maxilla linearis, integra. Labium nembranaceum, bifiduin.

93. Spondysis. Palpi aequales, fubfiliformes. Maxilla conica, integra. Labium corneum, bifidun. 46. Criuceris. Palpi aequales, filiformes. Maxilla bifita: laciniis cylindricis. Labium corneum, integrum.

14. Staphyunvs, Palpi-aequales, filiformes. La. bium trifidum.

10. Scavrvs. Palpi inaequales, filiformes. Maxilla obconica, unidentata. Labium truncatum, integrum.

5I. ZyGia. Palpi inaequales, filiformes. Maxilla unidentata. Labium elongatum, membranaceum. 
113. Meroe. Palpi inaequales, filiformes. Maxilla redta, bifida. Labium cylindricum, late emarginatum.

64. Horıa. Palpi zequales filiformes. Maxilia unidentała. Labium hifidum: laciniis linearibus, di. ftantibus.

14. Blaps, Palpi clavati. Maxilla recta, bifida. Labium membranaceum, fiffum.

17. Hezors. Palpi inaequales: antici fecuriformes, pofici clavati. Labium corneum, integrum.

I16. PaEDERvs. Palpi inaequales: antici capitati. Maxilla 'nidentata. Labiun lincare, integrum.

44. Cassida. Palpi inaequales: antici capitati. Maxilla finplex. Labium elongatum, integrum.

8. Opatrvm. Palpi inaequales: antici clavati: clava oblique truncats. Labium corneum, fubemarginatum.

73. Mordelra. Palpi inaequales: antici clavati. Maxilla bifida. Lanium membranaceum, bifidum.

II5. Oxyrorvs. Palpi inaequiles: poltici clavati. Labium marginatum, cum mucrone.

45. Chrysomera. Palpi fex filiformes. Labium corneum, integrum.

I. Scarites. Palpi fex filiformes. Labium corneum; bififin: Iaciuis difantibus.

********** Antennis filiformibus.

53. Apalvs. I'alpi aequales, filiformes. Maxilla cortre: , unidentata. Labium truncatum, integrum.

13. Manticora. Palpi filiformes. Maxilla cornea arm cuata. acutillina. Labium cornenm, trifidum.

13. Pialecia. Palpi filiformes. Maxilla menbranacea, undentata. Labium membranaccum; unidentatum.

25. Gyrinvs. Palpi requales, filiformes. Maxilla corusd, unidentata, acutiffima. Labium emarginatum.

66. Cvcrivs. Palpi aequales: articulo ultimo truncato, cralliuri. Labium brevé, bifidum: laciniis difantibus. 
22. Sconyrvs. Palpi acquales, filiformes: articulo ultimo obconico. Maxilla cornea, acuta integra. Labium breve, membranaceum acuminatnm.

4I. Heterocervs. l'alpi aequales, filiformes. Maxilla unidentata longitudine palporum. Labium membranaceum, late emarginarum.

56. Cryptocephalvs. Palpi aequales filiformes. Ma. xilla membrariacea, unidentata. Labium corneum, integtum.

97. Brvchvs. Palpi aequales, filiformes. Maxilla membranacea, bifida. Labium acuminatum.

34. Prinvs. Palpi aequales, filiformes. Maxilla bifda. Labium bifidum.

37. Parnvs. Palpi clavati : clava orbiculatav Maxilla bifida. Labium emarginatum.

69. Omalysus. Palpi extrorfum crafliores. Maxilla membranacea, bifida. Labium membranacenm, emarginatum.

57. Hispa. Palpi in medio craftiores, Maxilla bifida. Labium integrum.

71. Lycus. Os roftro cylindrico inflexo. Palpi artictre lo ultimo craffiori, truncato.

49. Cевrio. Palpi inaequales, filiformes. Maxilla unidentata. Labium membranaceum, apice palpigeruin.

30. Malachivs. Palpi inaequales, filiformes: articulo ultimo fetaceo. Maxilla unidentata. Labium membranaceuin, rotundatum.

78. BVPRESTIS. Palpi inaequales, filiformes: articulo ultimo obtufo, truncato. Maxilla unidentata. La. hiun acuminatum.

50. Cistela. Palpi inaequales, filiformes. Maxilla unidentata. Labium membrasaceum, bifidnm.

12. Seridivar. Palpi inaequales, filiformes. Maxilla unidentata. Labium breve, .. corneum, emarginatums. 
9r. Necydalis. Palpi inaequales, filiformes. Maxilla unidentata. Labium porrectum, membranaceum, late enlarginatum.

55. SAgRA. Palpi inaequales, filiformes. Maxilla cornea, unidentata. Labium breve, corneum, cilia. tum, fifum.

70. P'хвоснкол. Palpi inaequales, fubfiliformes. Maxilla integra. Labiun corneum, lineare, integrum.

68̧. LAMPYRIs. Palpi inaeguales, fubclavati, Maxilla bifida. Labium corneum, integrum.

2y. Cantharis. Palpi 'inaequales, fecurifornies. Ma. xilla bifida: Labium membranaceum, integrum.

79. Elater. Palpi inaeguales, clavati. Maxilla obtufa, unidentata. Labiun bifidum.

86, Calopvs. Palpi inaequales; antici clavati, Maxilla bifita: Lahimm bifidum.

62. Lyтta. Palpi inaequales: ponici clavati. Maxilla bifida. Labiun membranaceum, truncatum.

65. LYMEXYLON. Palpi inaequales: antici porrecti: articulo penultimo magno, appendiculato, fiffo, ultitno arcuato, acuto.

6o. Lagrias. Palpi inaequales; antici fecuriformes, pofici clavati. Labium membranaceum, integrum.

59. Trelvs. Palpi inaequales: poftici fecuriformes. Lao bium inter palpos rotundatum, integrum.

59. DRrops. Palpi inaequales; antici fubfecuriformes, pollici filiformes. Labium membranaceum, late emarginatum.

54. Atyrenvs. Palpi fex acuti, breviffimi. Maxilla fornicata.

47. Galli:rvga. Palpi fex: articulo ultimo acuto. Labium bifidum.

19. Carabve, Palpi fex: articulo ultimo obtufo, La. bium truncatum, integrum.

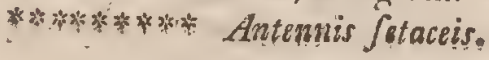


74. Donacia. Palpi aequales, filiformes. Maxilla uni. dentata. Labium cylindricum, integrum.

83. Cerambrx. Palpi aequales, filiformes: articulo ultimo fetaceo. Maxilla unidentata. Labium bifidum.

82. Prionvs. Palpi acquales, filiformes. Maxilla cy. lindrica, integra. Labium breviflimum, nembra. naceum, rotundatum.

88. Saperda. Palpi fubaequales, filiformes. Maxilla bifida. Labium cordatum, truncatum.

84. Lamis. Palpi úbaequales, filiformes. Maxilla cornea, bifida. Labium corneun, bifidum.

90. Leptvra. Palpi inaequales, filiformes: articulo ultimo truncato. Maxilla unidentata. Labium nembranaceum, bifidum.

92. Mozorchys. Palpi inaequales, filiformes. Maxilla bifida. Labiun membranaceum, bifidum.

52. Zonitis. Palpi inaequales, filiformes. Maxilla elongata, integra. Labium emarginatum.

89. Calzidiv m. Palpi aequales, fubclavati. Maxilla bifida. Labium bifidum: laciniis tenuifimis.

87. Rhagivm. Palpi inaequales, capitati. Maxilla unidentata. Labium bifidum: laciniis rotundatis.

85. Stenocorvs. Palpi inaequales: poftici clavati.

20. Cicindera. Palpi fex filiformes: poftici pilofi, multi articulati. Labium corneum tridentatum.

2I. Ezaphrvs. Palpi fex filiformes. Labium rotundatum apice acuminatum, integrum.

23. Dytiscrs. Palpi fex filiformes. Labiun corneum, truncatum, integrum. 


\section{E LEVTERATA.}

Letнrvs. (Kolbenkïfer.) SCARAB. Actaeon $L$. cephalotes Eur. -aeneus Inf. Maris Pacif. Scarabaevs. (Dungkiffer. Scarabé. Bectle. Bugg.) - Scutellati thorace cor. nuto.

Hercules $L .{ }^{\circ}$ ) Ass.

a) piceus.

B) glaucus. Alcides Ind. B) Perfeus O? Gideon L. 1nd. Oromedon Ind. Centaurus Afr. Aegeon Ind. difpar Ro/f. Chorinaeus Braf. dichotomus $L$. Ind. claviger $L$.Ind. haftatus Am. Enema Ind. Pan Ind. bilobus L. Eur. Daedalus Ind. truncatus $N . Z \ell$. Zoilus Caj.

a) Hanneton terreftre. Her. enle. Bectle Hercules. Todidi Am.

a) viger.

B) glaucus: Simfon L. Ind. Elephas Guin. Boas Sierra Leon: Coryphaeus C. B.S. bicornis Am. Orion Seneg. Tityus L.'An. Marianus L.

$\gamma)$ niger. Atlas L. Am. Geryon Ind. Aloeus et L. An. Typhon Vulcánus Guadal. Antaeus $A m$. Syplax $A m$. Maimon Am. 'Typhoeus L. ')'

B) longicornis.

y) bimáculatus. Mornus Ind. Titanus $A n b$. Aenobarbus Am. SCA. Fly.

b) Le Phalangifte. 
Scarabaevs, maficornis $L$. Scarabaev's. Aygulus

Eur. ${ }^{\text {) }}$

Sylvanus Braf.

Lazarus Am.

quadrifpinof. $C \mathrm{cj}$.

quadridens Ind.

mobilicornis Eur.

Cyclopss Ind.

Milon Braf:

अ: $\int$ cuttelluti thorace inermi,

capite cormuto.

Rhinocerus L. $A f$.

Augias Guin.

Barbacolla $N$. Holl.

Satyrus $A m$.

Jamaices:fis.

Silenus Eur.

Syrichtus $A m$.

Apelles C. B.S.

Hylax $A f r$.

Aries C. B.S.

Clinias Eutr. Hung.

Monodon E. Hung.

coronatus Jav.

Dionyfius Tranqueb.

Meliboeus Am.

didymus $L$. Am .

Valgus et L. Caj.

hircus Tranqubar.

punctatus Ital.

farctus $P$ enfylv.

ß) Cephus $M$.

retulus C. B.S.

piceus Ind.

Inuus Afr.

c) Nasborikifer. Le Nafisorne. Moine.
Ind.

fubterraneus L. Eur. ${ }^{d}$ )

Sorex Int.

Foffor $L:$ Eur. ${ }^{\circ}$ )

Scrutator Eur.

fulcatus Germ.

foetens Germs.

analis Ind.

fcybalarius Germ.

terreftris Germ.

obfcurus Germ.

ater Germ.

Porcus Germ.

bimaculatus Germs.

teftaceus Eatr.

fimetarius $L . E_{u r}{ }^{\text {f }}$ )

conflagratus Geran.

erraticus L. Eatr.

confpurcatus L. Ezw.

inquinatus Germ. .

B) ater.

futuralis $A m$.

fordidus Eur.

$\beta$ ) 4 punctatus:

luridus Eurr.

granarius Eur.

haemorroid. Eur. ')

nitidulus Eur.

marianus L. Ann.?

**a* fout

d) Erdrolubler Le Souterrain.

e) Der Grulkäfer. L.e Fof. foyeur La tête armée.

f) Le Scarabé bedeall.

g) Der Rothafser. 
***: Scutellati mutici, capite S C A R A B A E V s. falciatus thoraceque inermi.

Scarabaevs. lougimanus L. Ind.

ftercorarius $L .{ }^{\text {h) }}$ Eur.

B) viridis

y) fufco callaneus

8) bimaculatus. vernalis $L$ Eur. $\left.{ }^{i}\right)$ cordatus Gunial. excavatus Neap. Talpa Ird. Invencus $A m$. fplendidus $A m$. veter $I n d$. Iaborator $B r a f$. arator $C . B . S$. globator C. B.S. 7 unacilatus $A / r$. 2. punclatus Roff. rufipes L. Hur. nigripes Eur. lutarius Germ. Balkburuii Am. cont.minatus Germs.

B) imnaculatus. 4 maculatus Eur. 4 puftulatus Germ. Sus Germ. Kantećn. teftudinarius Angl. Scrofa Germ. merdarius A. L. Fur. quisquilius L. Eur. . melanocephalas S. $N$. Pecari Hung. deprefliss A. Germ. porcatus Germ. Aercorator $A m$. alper Eur. rufus Suec. arenarius Suec. foetidus Germ. Oedipus C. B.S. Rliadanifius Corom. nemeftrinus $C . B . S$. Iachus $C . B . S$. haltator $S . N$. Sabacus Coroms. Nanus Tianqb. fplendidulus $A m$. feftivus $L$. Am. Pactolus Braf. Pithecius Ind. Senicu'us Tranqb. metallicus S. $N$. Guineen lis, elevatus Enr. plagiatus L. Suec.

B) unicolor. marginellus Corom.

Dung Beetle.

h) Le grand pillulaire.

i) Le petit pillulaire,

pygmaeus Tranqb.

Catta Corom.

Rofalius Am. * Ex Ex cut. thor. cornuto. Ammon $A m$, Midas $A m$. Hamadryas C. B.S. Scara. 
Scarabaeys, lunaris 'L. Scarabaevs. Vacca $L$.

Eur.

emarginatus $E u r$. caelatus $A . C, B_{\mathrm{r}} S$.

Belzęul $A m$.

bifafciatus Corom.

Sinon Afr.

Faunus Cajen.

Pirmal S. $N$.

Tarandus Ind. capucinus Ind. :

Lemur Germ.

Camelus Germ. unifalciatus Tranqb. Aefon (nec Boas) Ind.

Antenor Seneg; ****** Ex cutellati thorace inermi capite cornuto.

Bonafus Ind.

Bifon Eur.

fagitrarius C. R. S.

Bucephalus Ind.

Mololfus L. Chin.

lancifer $L$. Ans.

Panifcus Am.

Carolinus L.

Nifus Cajent.

orientalis Ind.

Plutus A. Ind.

Sphinx Am.

unguiculatus Seneg.

Nicanor Ami.

Fricator Ind.

Mimas Anr.

Taurus L. Germ. ")

k) Fliegende Ocbs. Le Pioufier à cornes retrouffées.
Germ. ')

Capra Germ.

Ibex Ind.

Alces Hung.

Gazclia Grint.

Oryx Chin.

nuchidens $S$. $N$.

Tragus Chin.

Carnifex Am.

tridens Afr.

hifpanus L. Eur.

Moplus ind.

Spinifex Corom.

Cornobita Germu.

nuchicornis L. Eur. ${ }^{\mathrm{m}}$ )

Xiphias Germ.

nutans Germ.

aeneus Tranqb.

thoracicus Seneg.

centricomis $S . N$.

fcabrofus Surin.

furculus $S . N$.

furcatus Eur.

verticicornis Angl.

fulcator Cajen.

quadritiotatus (nec qua. dripuft.) N. Holl.

reflexus Chin.

Hybneri Hung.

quadridentatus $S . N$.

SCARA-

1) Le Boufier à deux corts nes.

m) Der Stachelnacken, Sca. rabé à nuque epineufe. Le pes tit Bonfier noir cornu. 
Scarabaevs. criffatus Alo Scararaevs. politus gypti. *****:**: Exfcutellati mutici. facer T. Eur. laticollis L. Eur. femipunctatus if $r$. variolofus Fumme. miliaris $I n d$, morbillofus Germ, profanus Guin. Bacchus $C . B . S$. gibbofus $A m$. Hollandiae $N . H$. I.eei Ind. muricatus A. Am. Koenigii Tranqb. granulatus. cupreus $A f r$. flagellatus C. B.S. fcabratus A. C. B.S. Schacferi L. Germ. voluens Eur. pillularius L. Eusr. obliquus Senegl. fqualidus Braf: Schreberi L. Eur. " ) giabratus $A f r$. 2 puftulatus $N$. Holl. pallipes Corom. triangularis Surin: atricapillus Am. cindtus Chin. fex punctata Cajent. ruges. Porro:

n) Le Boufieur à pointes

I) $\cdot \mathrm{Sc}$. anftriacus.

2) Sc, conjugatus.

S. $N$. flavipes Germ. fmaragdulus $A m$. difcoideus Afr. minutus $C$. $\dot{B} . S$. aterrimus S. $N$. ovatus Eur. pufillus $\mathrm{S}$. $N$. variegatus $\mathrm{S} . N$. parvulus S. $N$. 257. fufcopuncletus S. $N$. HEXODON. retictlatum Madag. unicolor Madag. His TER. (Stutzkäfir. Efcarbot.) maior.

B) maximus $L$. Afro unicolor L. Etkr. ot Ann. $\left.{ }^{\circ}\right)$ imprefius S. $N$. glabratus Germ. femipunctatus $A f r$. fcaber Hijp. cyaneus $N$. Holl. planus Eur. brunneus Suec. pygmacus $L$. depreffus. 4 dentatus $A m$. maxillofus $A m$. fulcatus Germ. oblongus Suec. abbreviatus Am.

\section{Hister}

a) L'Efcarbot noir. 
His ter. finuatus Germ. cruciatus $A f r$. bipuftularus $N$. S. 2 maculatus Em. ${ }^{\text {P) }}$ 4 inaculatus $A m$. aeneus Eur. detritus $N$. Holl. picipes Germ.

25. caelus $G$ rrm.

SP H A E I D I V M. (Kugelkäfer.)

Scarabaeoides Eur. ')

Dermeftes $L$. lunatum Germ. 2 puftulatum Germ. colon Suer. globus Gall. luteum Suec. dytifcoides I. St. Helen. glabratum Germ. $M a$ dera.

5 maculatum S. N. humerale Silec. abdominale $A m$. marginatum Germ. ferrugineum Germ. atomarium Germ. melanocephalum Eur. Dermiefies $L$. lugubre Gall. ftercoreum Geriss. falciculare Gall. haemorrhoidale Eur. flavipes Seland. ges.

p) Efcarbot aे taches rou-

q) Efcarbot d' ecaille.
S H A E R I D I $M$. Uni: punctatuar Eur. Coccionella $L$. limbatum Gerrn. ruficolle Germ. nigripenne Germ. bicolor Germ. fimetarium Eur. teltaceum $A m$. aeneum Germ. femiuulum Eur. minutum Angl. Silpha atomavia $r$. 31. pulicarium Eur. Silpha puicoria $Z$.

Byrkhvs. (Fugenkäjer.) gigas Germ. pilula L. Eur. ' $)$ albopunctatus Germ. fafciatus $L, E u *^{3}$ ) ater Germ. ") dorfalis L. Germ. muriuus Gern. varius L. Angl ") femiftriatus Germ. aeneus $E u r$.

BXR.

r) Ciftele Pilule.

s) d. bandirte Fugenk. La Ciftele à bande.

tj La Ciftele noir liffe.

ii) Der grün und flinarz fcbillirrde Fugnnk. La Ciftele fatinée smplius:

Byrrhus ornatus Germ.P. - fafcicularis

Germ, $P$.

- nitens Germ.P. 
BY R HVS. I I. minutus ERodivs. teftudinarius $C$.

Germ.

TRox. (Erd/taubkïfer.)

gemmatus Seneg.

fabulofus Eur. ')

- Scarabaeus L.

arenarius Germ.

fuberofus Braf:

horridus Ind.

luridus C. B. S.

cornutus Ceyl.

8. fpinicoruis.

Opatrvm. (Sandgrïber.)

grifeum Ital.

porcatum Rarb.

fabulofum Eur. Ann. w)

Silpha $C_{\text {: }}$

gibbum Suec.

depreflum $S . N$.

laevigatum Zeland.

crenatum Stier.

glabratum Ind.

arenarium C. B.S.

clathratum Cajenqua.

planum Sibir.

granulatum Barb.

orientale.

tibiale Suec.

frigatum $S . N$.

Agricola Germ.

minutum Suec.

18. pufilluns. Hung.

Eronivs.

v) Le Sablier. Le Scarable à larges cuiffes.

w) Le Tenebrion à Aries denteléess
B. S.

gibbus Arab.

planus Arab.

minutus Orient.

Scivrvs.

atratus Aegypt.

friatus Ital.

fulcatus Gall.

SCARITEs, (Schlupfkäfor.)

depreflus Cajen.

marginutus Cajenn.

teftaceus $A$. Senzg.

gigas $A f r$.

fubterraneus $A m$.

laevigatus 1 tal.

crenatus Ind.

cyaneus $N$. Eoll.

fabulofus Barb.

arenarius Eur.

Tenebrio fo/for $L$. gibbus Germ.

curfor Eur.

Tenelvio $L$.

I 3. Arabs Oriant. ${ }^{*}$ )

SePidiva.

tricu[pidatum Arab.

variegatum Barb.

criftatum Arah.

reticulatum C. B.S.

rugofum $C . B . S$.

6. vittatum C. $B . S$.

Pimelia. (Hürkerkäfer.)

PIME.

x) Species novae:

1) Sear. piceus, $P$.

2) Scar. gagates, .P. 
Pimlicia. ftriata Ind. unicolor C. B.S. flavicollis A. C. B.S. gibba Ind. laevigata Hung. glohofa A. C. B.S. glalurata Germ. hifpida Arab. longipes Aegypt. ruftica A. C. B. S. muricata Ear.

Tenebrio $L$. tuberculata Ital. variolofa A. C. B.S. 2 punctata ltal. fcabra C. B. S. grofla $B a r b$. angulata Albx. echinata C. B. S. dentipes $C . B . S$. dentata C.B.S. porcata C. B. S. maculata $C . B . S$. ferrata $C . B . S$. minuta $C$. $B$. Se rugola Aggypt. fpinofa Eur. Tenebrio $L$. acuminata Enw. reflexa Oricut. morbilio?a. S. $N$. fafciata Ind. collaris Eus: Tenebrio $L$. carinata Eur. ciliata C. B. S.
Prmelia. lineata Sibir. leucographa Eur. glabra Aegypt. anguflata Orient. 38. orbiculata Orient. BL A PS. (Todtenkäfer.)

Gages Eur. Tenebrio $L$. fulcata Orient. mortifaga Eur. ${ }^{y}$ )

Tenebrio L.

- excavata Corom. granulata C. B.S. bupreftoides $C . R . S$. dermefloides Eur. friata Corom. emarginata Marocco. dentipes $C$. B. S. variolola $A f r$. laevigata Maroc. triftis Barb ferrata C. B. S. foemoralis Germ. tibialis C. B. S. crenata. Corom. glabra Eur. punclata $A m$. 20. clathrata. $A m$. Tелевкіо. (Mehlkrifer.). cupreus Guin. fulcatus Guin. cyaneus A. Seneg. curuipes Germ.

y) Der Todtenpropbet. Der Stinker. Le Tenebrion-liffe à prolongentent. 
Tenebrio. ferratus $A f r$. oblcurus Germ. molitor L. Eur. laevigatus $A f r$. chalybeus Guin. variegatus $A f r$. abbreviatus Ind. depreflus Carol. follor C. B.S. cornutus Smimae. fanguinipes $N$. Holl. mauritanicus $L$. Ann. brunneus $\mathrm{S} . N$. culinaris Hifpar. pallens $C . B . S$. cadaverinus Ezur. villofus Ear.

22. filiformis, Barb. $^{z}$ )

Tragosita. (Kneipkäfer.) coerulea Gall. bicolor. S. $N$. caraboides Eur. ") Tenebrio $L$. virelcens $A m$. bidens Gall. thoracica Lapp. fulcata Gall. ferruginea $A f r$. HELops. (Schattenkïfer.) laminatus Ind. punćtulatus Ind. coeruleus Ear.

Tenebrio L. gineus $P$.

z) Forro Tencbrio ferru.

a) La Chevrette brume.
Helops. lanipes Germ. Tenebrio L. micans S. $N$. marginatus Güin. aeruginolus $C . B . S$. bicolor $A m$. ferratus Eur. Chryfonela caraboides $L$.

batbatus Germn. canaliculatus Germ. haemorrhoidalis Ind. luridus Braf. laevis Gorm. equeftris Braf. maurus 1nd. morio Anr. nigrita $A m$. picicornis Ind. rufipes $N$. Holl. longipes Afr. capenfis $A$. ater Gervis. marginatus A.C. B.S. Pimelia Angl. Hottentotta Gall. femorata S. $N$. dentipes Coron. undatus Cajen. quisquilius Eur. Tenzebrio $L$. flavipes Anr. ruficollis Germ. 33. cyaneus Eur. ${ }^{\text {) }}$ ) Manb) Species novae:

I) Helops 
MA nticora. maxillofa $C . B . S$.

CAR A Bvs. (Laufkïfer. Cha/jeur des chenilles.)

* Thorace cordato. maxillofus $C . B$. $S$. thoracicus $C$. B.S. coriaceus L. Eur. ') glabratus Germ. marginalis A. Eur. violaceus L. Eur. purpurafcens Germ. catenulatus Germ. B) atroviolaceus. carolinus. cyaneus. Eur.

C. intricatus Li hilpanus. monilis Germ. gemmatus Germ. hortenlis $L$. Eurs.

B) aeneus. concolor Germ. irregularis Eur. taedatus $A m$. arvenfis Germ. hungaricus. retufus Pattag. lit. Maderae. convexus Germ. auratus L. Eus:

1) Helops triftis. $P$.

2) - fufcus, $p$.

3) - caraboides, $P$.

4) - picipes. P.

5) - fafciatus. P.

c) Le Carabe chagriné.
C A r A в vs. 'fplendens. Iamaic.

auronitens, Germn. futuralis Terradel Fute

- go. morbillofus Maurit. rugnfus Marocco. granulatus $L$. Eur. clathratus L. Eur. nitens L. Eur. roftratus Eur.

Tenebrio $L$. attenuatus Germ. elevatus Am. unicolor Am. attelaboides $I n d$. lencophtalmus $L$. Eur. ${ }^{d}$ ) planus Germ. ftriatulus Pattag.lit. arenarius Cambr. fabulofus $L$, Germ. lateralis Eur. ruficornis Eur. ')

B) pubefcens Müll. fulvipes Germ. picicornis Ital. flàvicornis Germ. piceus L. Eur. femoralis $A f r$. madidus Angl. terricola Germ. americanus.

I3 2

Cara.

d) Der roeifsäugicbte Lanfkäfer. Le Buprefte tout noir. louté. 
Carabus. Janus Carol. fumans $A m$. crepitans L.Eur. ')

B) Ininor.

Sclopeta Gall. fpinibarbis Angl. aencocephalus Am. Inf. humeralis Ital.

B) Diana F. Germ. variolofus $\Lambda$. Germ. axillaris A. Germ. trilobus Gwin. multipunctatus L. Eur. oblongopunstatus Eur. cinclus Corom. rufipes. nitidulus Kamtfch́. ruficollis Am. olens Ital. agilis Suec. falciatus Suec. 4 notatus. atricapillus L. Eur. marginellus A. Germ. 4 puftulatus $A f r$. 8 punctatus $S . N$. oblongus Germ. albipes (fallo oblongus) Germ. rubens Germ.

*: Thorace poftice anguflato. Io guttatus L. Afr. 6 guttatus Ind.

f) Der Bombardierkäfer. Der Plazzk. Le Bombardier. Carabé petard.
Cara B v S. venator Senze. gál. fulcatus Senegal. 6 maculatus Barbar. Nimrod Seneg. 7 guttatus A. C. B.S. Aclaeon (nec 4 guttatus) C. B. S. tabidus $C . B . S$. nigripennis A. C. B.S. variegatus Orient. exclamationis Barb. trilineatus Germ. cephalotes Eur. laevigatus Corom. intertruptus Orient. tricufpidatus $B a r b$. calydonius Ital. complanatus L. Domingo.

2 maculatus $L$. Ind. $O$. 3 puftulatus Sian. caliginofus S. $N$. 6 puftulatus Ind. $O$. variolofus Transf. **: Thorace quadrato. Atriola Germ. gibbus $\Lambda$. Germ. impreflus S. $N$. metallicus Germ. politus Ind. $O$. **:* Thorace rotundato, margine prominulo ob. tufo.

alternans $A m$. $I n)^{\circ}$ : calidus $A m$. $1 n j$. 
Carabys. fericeus Germ. Carabve. abdominalis $C$. porcatus $N$. Holl. reflexus Corom. polticus $\mathrm{S} . \mathrm{N}$. angulatus, Corom. caffidens Gall. filphoides Gall. globolus Marocco. indagator Barb. Sycophanta L.Eur. ') inquilitor $L$. Eur.

ß) niger.

Scrutator Virgin. reticulatus Eur. longicornis Marocco. brevicollis Germ. punctulatus Germ. micans. obfeurus Gervis. fulcatus A. C. B. S. bicolor. 'Am. holofericeus Germ. 2 notatus Germ.

B) nigripes. punctatulus Barb. fulvicollis Barb. pilicornis Angl. cuerulefcens $L$. Eur. lepidus Germ. laetus A. Eur. Schich Am. Inf. cupreus L. Eur. carnifex Terra del Fuego. vulgaris L. Eur. integer S. $N$. latus L. Eur.

g) Carabé Sycophante.

B. $S$. ovatus Germ. helopioides Germ. azureus Gem. erytrocephalus Germ. analis Girm. lineola Arr. ferrugineus $L_{\text {. Eur. }}$ pallens Germ. Surinamenfis. St. Crucis S. $N$. dorfiger Barb. aterrinus Etu. ") aeneus Germ. nigricornis Dan. parum punctatus Germ. 6 punctatus L. Eur. i) palliatus S. $N$. difcoideus Germ. marginatus $L . E_{t u r}$. veftitus Hung. ${ }^{k}$ ) nigrita Germ. fufcus Germ. pallipes $A n$. quadrum Seneg. melanocephalus $L, E u r .{ }^{1}$ ) cyanocephalus L. Eur. rufibarbis Germ.

B 3

Cara-

h) Der ganz fchuarze Lk. mit 3 Hoblpusksen auf den Flizgeldecken.

i) Carabé à fix points. Le Puprefte à etuis cnivereux.

k) Le Bupr' vert à bordure.

l) Carabé tête noir. L.e Bü. prefte noir à coscelet rutuge. 
CAR A B s. amethyflinus

\section{Cajen.}

flavilabris S. $N$.

lividus Dan.

crux major. L. Etwr. ${ }^{m}$ )

crux minor. L. Eutr. ")

vittatus Am. B.

turcicus.

haemorrhoidalis Germ.

picipes Suec.

2 puftulatus Angl.

Andreac 1tal.

elevatus Gall.

germanus $L_{*}{ }^{\circ}$ )

Velox Eur.

rufefcens $A n g l$.

praeuhlus Germ. apricarius Dalec.

lunatus Angl, ${ }^{\prime}$ )

prafinus Dan.

Curfor Ital.

furcat us $A m$.

vapòrariozum $L$. Eur,

meridianus L. Eur.

difous Gern.

comma Am.

4 maculatus L. Equr.

4 guttatus Angl.

ufulatus $L_{\text {. : Eur. }}$

dorfalis Germ.

2 guttatus Norw.

iin) Das grofse Kreuz. Le. clevalier noir.

ii) Das kleine Krenz Le Chevalier rouge.

o) Die Vinlezfritze.

p) Der Ritterlaufkäfer.
CARABV S.' fmaragdulus

S. $N$.

guttula Germ.

feneflratus A. Germ.

teflaceus L. Eur.

truncatus Germ.

abbreviatus Noru.

2 punctatus L. Eur.

celer Eur.

minutus Germ.

pygmaeus Germ. trillis Germ.

truncatellus L. Eur.

2 I 4. minimus Germ. q)

Cxchrvs. (Derkkäfer.)

roltratus A.' Eur.

Tenelvio $L$.

attenuatus A. Germ.

Cicrndela. (Sandkäfer.)

longicollis Siam.

aptera $I_{n d}$. $O$.

CiCIN.

q) Porro hic referuntur:

1) Carabus terminatus, $\mathrm{H}$.

2) - cifteloides. $\mathrm{H}$.

3) - fpoliatus. P.

4) - zonatus. $P$.

5) - depreffus. $H$.

6) - feftivus, P.

7) - pumicatus, P.

8) - vernalis $P$.

9) - inaequalis. $P$.

10) - latefcens. P.

I1) - articulatus. P.

12) - confluens. P.

I3) - fabulicola. P.

14) - longipes, $P$.

15) - impreffis. $P$. 
CICINDELA. attelaboides

A. Ind. Or. anguftata Germ. 3 pultulati Gall. grofla Corom. cyanea 1 Ind. $O$. megacephala Senrg. campefris L. Eur. hybrida $L$. Eur. ') finuata. fylvatica $T$. Euw. arenaria Barb. littoralis $B a r b$. triftis. interrupta Siesr. $L$. lunulata. lurida. chinenfis. Alexuofa Hifpan. capenfis $L$. germanica $L .{ }^{\text {s) }}$ oblcura S. $N$. micans S. $N$. tuberculata $N$. Zeland. unipunztata Am. 2 punctata. 6 punctata Malab. B) aurulenta. 4 lineata Ind. cincla $A f r$. biramola Ind. 6 guttata $V$ irg. catena Ind. 0 . longipes S. $N$.

r) La Cicindele tachetée.

s) Le Buprefte vert i fix points blanches.
CICINDEL A. marginata Virg. 8 guttata $A$ An. 3 fafciata $A m$. angulata $\mathrm{S} . N$. Carolina $I$. virginica. cajennenfis. emarginata Gall. aequinoctialis Surin. maura L. Orient. 45. minuta Ind. ELAyHRvs. (Strandkäfer.) uliginofus Germ. riparius H. Eur. ${ }^{t}$ ) friatos Germ. flavipes L. Eur. ") aquaticus Eun.")

Cicindela $L$.

femipunctatus Germ. 2 guttatus Norm. " ) Scoly's S. (Sumpfkäfer.) flexuofus Ind. $O$. limbatus Germ. ${ }^{x}$ ) HYDROPHIL. (Schwimm. kïfer.) piceus Eur. ${ }^{\mathrm{y}}$ ) Dytifcus $L$. olivaceus Corom.

B 4

HYDRO=

t) Cicintdele de rivage. ii) Cicintele à pattes jaunes. lée.

v) Le Buprefte àtête canc

v) Porro:

Elaphrus paludofus. P.

x) Scolytus aeneys. P.

y) Le grand Hydrophile. 
Hydrophilvs. ater Am. D x T I c v s. ruficollis $M$.

caraboides Eur. Dytifcus $L$.

lateralis $A m$. $M$. rufipes Chin. emarginatus Eurr. Scarabaeoides Eur. Dytifczss $L$. picipes Germ. orbicularis Germ. fubrotundus $A m$. bicolor Dan. collaris Am. $M$. undatus $A m$. $M$. erythrocephalus. haemorrhoidalis Germ. marginellus Germu. obfcurus Gern. luridus Eur. ${ }^{2}$ ) Dytifcus $L$. minutus Eur. grifeus Germ. bipunctatus Suec. Dytifcus fuscipes $L$.

23. pygmaeus Aim. $M$.

DY'Iscvs. (Waderkïfer.) latillimus L. Eur. ") coftalis Surin. marginalis L. Eur. . punctulatus Germ. immarginatus A. Seneg. Roefelii Gall. limbatus Chiss. latcralis S. $N$.

z) Hydrophile brun.

a) Ditique large,

Siam.

fulcatus L. Eur. Am. b) fafciatus Ind. friatus L. Eur. fufcus L. Eur, Lanio Mader. cicurus C.B.S. vittatus Ind. cinereus L. Eur. grifeus Ind. niclicus $L$. Afr. 10 punctatus $N$. Holl. fuliginolus Ital. 2 puntulatus L. Eur. cinctus Am. 2 punctatus Germ. feneftratus,Germ. Hybneri Germ. fagnalis Germ. transverfalis Eur. calidus Am. Hermanni Gall. abbreviatus Germ. gibbus Germ. uliginofus $L$. Eur. irroratus Amo agillis Germ. maculatus L. Eur. erythrocephalus L. Eur. planus Dan. varius Patag. notatus Germ.

Dytis-

b) Mas. Le Ditique à cor. celet a bandes. Femina Le Dy. tique filonnée. 
Dytiscvs, haemorrhoida- Gyrinvs. (Tauchküfer.)

lis S. $N$.

rufipes.

depreflus Suce. dorfalis Germu.

B) rufifrons F. Gaill. 6 pußtulatus Suec. palufris L. Eur. ovatus L. Eur. picipes Germ.

B) bicolor Guin.? lituratus 1 tal. chryfomelinus Germ. fignatus Patag. I 2 puftulatus. 8 puftulatus Suec. halenfis.

granularis $L$. Eur. confluens Germ. obliquus Germ. impreflus Eur. femipunctatus Ind. $O$. crux Ital. geminus Germ. lineatus Gall. inaequalis Suec. minutus $L$. Eirur. pygmaeus Dan. reticulatus. craflicornis Germ, flavipes. Ind. $\dot{O}$. pictus Gern. nigrita Germ. pufillus Ital. 72, parvulus $D a n .{ }^{c}$ )

c) Nosae fpecies comme. morantur, fequentes: natator $L$. Eur. ')

bicolor Suec. aufralis $N$. Holl. americanus. micans Guin. fpinofus Corom. 7. ftriatus Barb. ELOPHORVS. (Wa/)errunzelkäfer.) aquaticus Germ. ${ }^{\text {) }}$ )

Silpha $L$. nubilus Germ. elongatus Germ. humeralis Germ. flavipes Suec. crenatus Germ. pygmaeus Gerna. 8. minimus Eur.

Clervs. (Ameifenkäfer.) mutillarius Germu. dubius Am. ichneuinoreus $A m . B$. Sphegeus $A m$. $B$. formicarius Eur. ${ }^{\mathrm{f}}$ ) Attelabus $L$.

B 5

Clerves.

1) '́ytifeus interruptus. $\dot{P}$.

2) - collaris. P.

3) - obfcurus, P.

4) - arcuatus. P.

5) - trifidus. $P$.

6) - elegans. P.

7) - elcvatus, P.

8) - Volckmeri.P.

d) Le Tourniq̨uet nageur.

e) Le Dermefte bronf́́,

f) Le Clairon fourmi. 
CLER V S. $\sigma$ guttatus Ams.

4 inaculatus Germ. unifafciatus Eur. 8 punctatus $H i f p$. tricolor Afr. 2 fafciatus Sibir. fipylus Afr. Ammios iffr. apiarius Germ. B) Aitolabus L. alvearius Eur. ") cyaneus Ind.

I 7. crabroniformis Orient. Notoxvs. (Schildkrötenkiäfer.) porcatus Ter. Diem. violacens $N$. Zel. 2 fafciatus Lapp. dubius Germ. indicus $\Lambda$. chinenfis. mollis Eur. ') Attelabus $L_{\text {s. }}$ monoceros Eur. ") Attelabus. $L$. cornutus Ital. 2 punclatus Germ. antherinus Eur. Meloe $L$. floralis Eur.

g) DerBienernolf. Le Clairon apivore.

h) Der Bientizellenkäfor. Le Clairon à bandes ronges.

i) L.c Cla.1ron porte-croix. k) La Cuculle.
Meloe $\mathrm{I}$.

No т oxvs. I3. Ininutus Eur. ')

Cantharis. (Afterleuch. kïfir.)

fu[ca $E_{u t r}{ }^{m}$ )

livida $L$. Eur.

difpar Grrm.

analis Hung. viridelicens $C . B . S$. obfcura L. Ezrr. pellucida Germ. limbata Ian. lateralis L. Eur. fmaragdula Braj. atra $L$. Eurs. marginata $A m$. diadema S. $N$. 2 maculata $A m$. $S$. pallipes Germ. haenorrhoidalis Germ. pailida Germ. ruficollis Angl. flavipes Chin. melanocephala Corom. falciata Ind. $O$. 2 punctata Germ. lacta Ital. fulvicollis Germ. .

Can-

1) Specier novae funt: I) Notoxus ater. P.

2) - ferricornis, $P$.

3) - nectarinus, $P$.

4) - thoracicus. P.

5) - vlycinus. P.

6) - caftaneus. P. m) La Cantharide ardoifé. 
Cantharis. lineola Ind. Ma lachivs. praeufus

$O$.

nigripes Eur. melanura $L$. Evr.

B) melanocephala.

vittata Carol. nitidula Germ. Morio Germ. nigra Eur.

Dernieftes $L$. pulicaria Germ. ninima $L$. Eur. teftacea L. Eutr.

2 guttata L. Eutr. ${ }^{n}$ ) 36. Cardiacae Silec. .

Cantharis $L$.

Malachivs. (Warzenk.) aeneus Evr. ${ }^{\circ}$ )

Cantharis $L$. puftulatus Eur. ${ }^{\mathrm{P}}$ )

Cantharis $L$. rufus Gall. marginellus Gall. viridis Scan. fanguinolentus Germ. ruficollis Germs.

cyaneus Hifp. viridipennis $C$. B.S. angulatus Germ:

n) Telephore à deux points jaunes. La Cicindele nnir à deux points jaunes et corcelet noir.

o) La Cicindele bedean. Telephore verd - bronze.

p) La Cicindele vert à points rouges. Telephore verd à deux points rouges.

\section{Germ.} pedicularius L.Eur.

Cantharis $L$.

pulicarius Germ.

falciatus Eur. q)

equeftris Ital.

Meloe $L$.

flavipes Germ.

albifrons Gall.

18. nitidulus Barb.

Melyris.

\section{viridis C. B.S.}

niger.

lineatus.

Derinestes. (Speckköïfer.

Jardarius $L .{ }^{\mathrm{r}}$ )

carnivorus N. Holl. et

Germ.

cadaverinus St. Hel.

macellarius Germ.

pellio $L .{ }^{5}$ )

undatus E. Eur.

3 fafciatus Eur.

20 guttatus Germ.

tigrinus Ital.

bicolor Germ.

vulpinus $C$. B.S.

felinus Terr. Dient.

Derme-

q) La Cicindele à bandes rouges. Telephore à bandes rouges.

r) Le Dermefte du lard.

s) Le Dernicte à deux po. ints blanes. 
DERMESTES. hirticollis Barb. murinus L. Eur. teflellatus Angl. violaceus Eur. *) rufipes $A f r$. ruficollis $I n d . O$. fanguinicollis Germ. Lycoperdi Germ. 2 punctatus Germ. finetarii Germ. fumatus L. Eur. tomentofus Angl. fulcus Germ. 6 dentatus Germ. lunatus Suec. unidentatus Gall. 2 deutatus Germ. picipes Gern. nigripes Germ. obfcurus Germ. teftaceus Belg. fcaber $N$. Holl. clinenfis. fcanicus $I$. limbatus $N . Z e l$. feneftralis L: Eur. variabilis Dan. Serra Suec. cellaris. crenatus S. $N$. minutus Dan. pedicularius $L$." Germ.

Vrticae Germ.

*) Le Clairon bleu.
DERMESTES. I 46. bra chypterus Germ. ss) A повгум. (Hauskäfer.) teffellatum Angl. friatum Germ. rufipes Germ. caltaneum Gall. pertinax Eur. Ptiuns L. Boleti Germ. ${ }^{*}$ ) B) teftaceun. molle Eur. Ptinus L. paniceum Eur. Abietis Germ. planum Germ. capenfe. minuturn Ital. inicans Germ. 14. nitidum Germ. ") Prinvs. (Bohrkiffer.) pubefeens Gall. germanus $L$. longicornis Germ.

Pri.

ss) Novae fpecies fequentes funt :

1) Derm. ferra. $P$.

2) - lonpicornis. P. tus. P.

3) - feunicoleoptra

4) - ater.P.

5) - porcatus. $P$. tus, $P$.

6) - quadripuftula-

t) Der Hauskiifer in den Lïberpilien.

i1) Porro: Anobium fe. fivum. $P$. 
Ptixvs. Fur $L_{0}{ }^{~}$ ) imperialis L. Eur. crenatus Germ. Latro Gall. denticornis Gall. ferricornis Am. fpinicornis Sandw. I. fulcatus Cannr. $I$. Scotias Eur. ${ }^{\text {w) }}$

13. friatus Germ. ${ }^{x}$ )

PT ILIN vs. (Federkammkäfer.) myfacinus $N$. Holl. pectinicornis Eur. ${ }^{\text {y) }}$ flavefcens Gall. ") peclinatus Germ. muticus $E_{u r}$. Hifpa $L$.

MeLASIS. (Fedirtrïger.) flabellicornis Angl. Elater $L$. myflacina A.C.B.S. Serropalpus. Hellen?

PAR N v. (Henfchreckeskääfer.) prolifericornis Suec. a) Elater $L$. acumiuatus Germb. obfcurus Germ.

v) Vrillette carnacicre. w) La Bruche fans ailes. tus, $P$.

x) Porro: Pt, fex puncta-

y) La panache brune. P.

z) La panacbe jaune. les.
N I C R OPHOR V S. (Todterm

gräber.) gerınanicus. b)

Silpha $L$.

B) punctatus. humator Eur: grandis $A m . B$. vefpillo Fur. ')

Silpha $L$.

B) minor. mortuorum Germ. Silpha. (Aarkïfer.) furinamenfis. littoralis L. Eur. micans $A$. livida Gern. . indica $L$. aınericanla. grofla L. Eurr. thoracica $L$. Eurr. laevicollis $N$. Holl. marginalis $A m$. B. rugofa L. Eur. ") atrata L. Eur. inaequalis $A m$. $B$. lunata Germ. laevigata Eur. obfcura L. Eur. B) ferruginea. reticulata Germ.

Sripha.

b) Bouclier grand noir.

c) L.eFoffoyeur commun,Le Bouclier folloyeur. Le Dermefte à point d' Hongrie.

d) Le Eotelier raboteux. Bouclier noir chifonne. 
Silpha. opaca L. Eurr. fiuuata Angl. Germ. 4 punctata L. Angl. paedernontana. ferruginea L. Iiur. oblonga $L$. Ezur. dentata Eur. S. feabra $L$. limbata Afr. undata Germ.

27. minuta Germ. e) Nitidvis. (Glanzkäfer.)

2 puftulata Eur. f) Silpha $L$. 4 pultulata Germ. oblcura Germ. abbreviata $N$. Zel.

B) nigripes. marginata 1 tal. aefiva Eur. Silpha $L$. obfoleta Eur. S. deprefa $L$. ferruginea Angl. Dermefles $L$. ftrigata Germ. imperialis Germ. Io guttata Germ. varia Germ. fordid: Germ. flexuofa Gall.
Nitidvea, bicolor Germ. Colon Eur. Silpha $L$. limbata Germ. hacmorrhuidalis Germ. difcoides Augl. pedicularia Eur. Dermeftes pfyllius $L$. 6 pultulata Germ. litura Grrm. fafciata S. $N$. floralis $A m$. $M$. aenea Angl. viridelcens Germ. hemiptera $A m$. $M$. dimidiata Am. Inf. truncata $G \bullet r m$. 30. rufipes Angl. B) Silpha $L$.

HETEROCERVs. (Mauhzourfskiffer.) marginatus Gernu, h) ANTHRENVS. (Knollkäfer.)

Pimpinellae Ezr.

Hifrio Germ.

Scrophulariae Eur. i) Byrrhus L.

Mufeorum. Byrrhus L. Verbafci Gall. Byrrhus L.

ANTHRE.

e) Species novae: la.

I) Silpha melanocepha-

2) - hemiptera.

f) Le Dermefte à delix po. ints rouges. g) Porro: Nitidula punctatifima. P.

h) Porro: Heterocerus laevigatis. $P$.

i) L' Anthréne à broderic. 
Anthrenvs. varius Gall. Coccinella. divularis pubefcens Germ.

hirtus Gurn.

ferraticornis St. Crux.

denticornis St. Crux.

Coccinella. (Marienkï-

fer. Vache \& Dien.

Béte de la vierge. La.

diy-cow. Lady-bird.)

marginata $L$. Ams.

limbata Germ.

marginella $A m$.

diaphana'S. $N$.

pubefcens S. $N$.

furinamenfis $L$.

immaculata Anı. Inf.

unicolor Ind. $O$.

M. nigrum Gerss.

difcolor S. $N$.

cincta S. $N$.

fanguinea $A m$. IM.

impunctata Stiec.

dinidiata Coroni.

margine punctata Germ.

lineola $N$. Holl.

unifafciata Germ.

annulata L. Germs.

trilincata $A m$.

futnralis S. $N$.

vittata Guinz.

ftriata Guin.

oblongopunctata RoJf.

abbreviata $A m$. $B$.

6 lineata $R o \iint$.

2 punctata L. Eur.

3 punctata L. Germ. hieroglyphica L. Eur.

Stzer.

tricincta Chin.

arcuiata Cíin.

undata $C . B . S$.

flexuofa Girm.

cingulata Tranqb. inaequalis $N$. Holl. trifafciata L. Eztr.

interrupta.

2 fafciata C. E. S.

4 notata Germ.

4 maculata Germ.

5 punctata L. Eur.

5 inaculata Germ.

6 punctata Eur.

glacialis $A m . B$.

6 maculata Ind. $O$.

7 punctata L. Eur. ${ }^{k}$ )

7 maculata Germ.

7 notata Germ.

8 pundtata Eur.

ß) 4 punctata L.

transverfalis Corom.

octomaculata.

9 maculata $N$. Holl.

9 punctata L. Eur.

10 punctata $L$. Eur.

Io maculata Am.

B) Chryfom.

10 maculata $M$.

innuba Orient.

dilatata Am.

B) chinenfis.

Cocci.

k) La Coccinelle rouge a 7 points noirs. 
Coccinelia. I I. punctata Coc CINELLA. 8 guttata

L. Eur.

I I maculata Hifp.

I 2 punctata Eur.

variegata $C . B . S$.

chrylomelina Afr.

borealis $A m$.

caflidea Maryl.

difpar Seneg.

(nec variegata.)

I 3 maculata Stiec.

I 3 punctata L. Eur. verficolor Chin.

B) vigra.

I4 maculata Germ. ocellata L. Eur. ') If punctata Ital. I 8 punctata L. Euvr.

I 9 punctata L. Eur.

20 punctata Angl.

22 punctata L. Eur.

22 maculata Guin.

23 punctata L. Gern. 24 punctata L. Eur.

24 maculata Tranqueb.

28 punctata Tranqb. conglomerata $L$. Eur.

B) 14 punctata L. conglobata L. Eur.

B) $I 6$ maculata $M$.

y) gennella $\mathrm{H}$.

lineola Am.

tricolor $\ln \int$. Amfterd. derrita $N$. Holl.

2 guttata Eur.

1) La Coccinelle rouge a 15 points noir.
Kamt fch.

I O guttata Susec.

bis 6 guttata Norw.

I 2 guttata Cajers.

14 guttata L. Eurr.

bis 7 guttata Germ.

I 5 guttata Germ.

I 6 guttata $L$. Evw.

I 8 guttata L. Eur.

20 guttata L. Eur.

oblongoguttata L. Eur. impultulata Germ.

nitidula Ans. $\ln \int$.

parvula Gall. ${ }^{\text {m) }}$

villofa Cajen.

analis Germ.

haemorrhoidalis Germ.

oculata $A$ m. $B$.

Cacti $L$. Am.

2 pufulata $L$. Evir.

variabilis Germ.

B)' lutea.

frontalis Germu.

4 puftulata L. Eur.

4 verrucata Germ.

bis 2 puntulata.

arctica Lnpp.

erythrocephala Gevm.

6 pufulata $L$. Eus:

Iunatis St. Hel.

I o puftulata L. Eur.

bis 5 puftulata.

(nec I o puftulata) Grs'm.

Coccr-

iii) Coccinelle velue à taches rouges au corcelet. 
Coccinelia, duodecim. pufulata S. $N$. quatuordecimpuftulata $L$.

Eur. guttatopuftulata $N$. Holl. felina Am. $B$. pantheriua L. Eur. pardalina. urfina Am. B. bonina N. Holl. tigrina L. Eur. 127. canina C. B. S. ${ }^{n}$ ). C A S S I D A. (Schildküfer.)

viridis $L$. Eur. ${ }^{\circ}$ ) equeftris Germ. affinis Germ. vibex Germ. C. virens $L$. cruenta Ind. $O$. auftriaca. nebulofa $L$. Eurr. atrata Germ.

n) Porro: P.
1) Cocciriella pubefcens.

2) - areata. P.

3) - Scyinnus qua. dripuftulatus. $\mathrm{F}$.

etatus. P.

4) - - bipun. ตแล. $P$.

5) - - nigri-

6) - lateralis. P.

7) - reppenfis. P.

$\therefore$ 8) - campentris. $P$.

-) La Caffide verte.

p) La Caffide panachée.
Cassida. Murraea $\dot{L}$. - Germ. ${ }^{p}$ ) 6) maculata L. vittata S. N. ferruginea Germ. limbata Germ. marginella Braf. cincta $A f r$.

hebraea Cajen. judaica Cajen. obfcura lrad. $O$. triftriata Surin. Annulus Cajen. notata $S . N$. 6 punctata Cajen. interrupta N. Holls. 8 punctata Siams. deufta N. Holl. cribraria Am. nobilis $L$. Eur. bicolor $S . N$. margaritacea Germ. cruciata $L$. Eur.

Crux Cajen. II punchate Cajen. finuata $A F$. arcuata $A m$. $M$. clavata $\mathrm{S} . N$. 2 notata Cajen. 2 tuberculata L. Cajen. tuberculata $A f r$. futural is C.B.S. 2 guttata Cajerr. miliaris St. Hel. 2 maculata Guin. punctata C.B.S. $c$ CASSI- 
Cassida. marginata $L$. Chrysorrera. puftulata.

Ind.

4 puftulata Cajen. dorfata Sinm.

Jamaicenfis $L$.

St. Crucis $A m$. Inf.

perforata $A m$. $M$.

bicornis Ind.

Taurus Cajen.

cyanea L. Ind.

fpinifex L. Ind.

bidens Braf.

gibbofa Braf.

truncata.

leucophaea $L$. Am. $M$.

flava $L, A m$. $A$.

reticularis $L$. $A m$. $M$.

variegata $L$. $A m$. $M$.

trifalciata $A m$.

reticornis Cajen.

annulata Ind.

grofla L. Am. $M$.

lincata $C . B . S$.

exclamationis $L$. Am. $M$.

inoequalis $L$. $A m$. $M$.

lateralis $L$. Am. $M$.

palliata Cajen.

difcoides $L$. $A m$. $M$.

2 puftulata L. Cajen.

cuprea Cajen.

6 puflulata Braf.

16 punctata $A m$. $M$.

1 74. 2 punctata Ind. orient.

CHRysomela. (Goldhïhn.

$$
\text { chesk) }
$$

punctatiflima Cajen.
Cajen.

Tenebricola Eur.

Tenebrio latvigatus $L$.

rugola $A f r$.

Tenebrio $L$.

Morio Terr. Dien.

coriaria Germ.

nigrita Gall.

Göttingenfis $L$. Germ.

hottentotta Germ.

aethiops Germ.

vittata $A m$. .

exclamat. S. $N$.

bicolor Alex.

affinis Barb.

lufitanica.

ferruginea $A f r$.

Bankfii Lufit.

metallica Germ.

lamina Germ.

obfcurata S. $N$.

gibbola $A m$. $M$.

8 inaculata Surin.

Io pufulata St. Dom.

trimaculata $L$. Am. $M$.

Adonidis Germ.

B) dorfalis $\mathrm{M}$.

clavata.

trifalciata Surin.

futuralis Cajen.

pulchra $A m$. $S$.

guttata C. B. S.

4 punctata L. Ind. $O$.

ignita Cajen.

furinamenfis.

Canxso. 
Chrysomela. afiatica. graminis 1.. Ettrr. bifrons ltal. cuprea Germ, q) triftis Eur. haenoptera Eu: varians Gernu. violacea $G$ erm. Centaurii Germ. tricolor Ving. luteicornis $A m, M$. femiftriata Braf. Populi $L$. tremula Ear. grofla Ital. faphylaca. L. Eur. fervida Iavae. polita L. Eur. lunata.

glabrata Surin. lurida L. Gall. linea (nec vittata). folida Am. M. nigricornis $N$. Holl. collaris L. Eur. Salicis Germ. fenegalenfis. viminalis $L$. Eatr. cyanipes $N$. Holl. cyanicornis $N$. Foll. Io punctata L. Eur. 6 punctata Ettr. pallida L. Eur. friata C.B.S. notata $C . B . S$. téa q) La Chrurfomele brique-
Chrysomela. alternans $A$. An. $[n$ f. Rumicis Hifp. vulpina C. B.S. craflicornis $N$. Holl. micans A. Sumatra. lapponica I. ') undulata $L$. . Iud. I \& guttata $N$. Holl. Iolygoni $L$. ruflica. brunuer $N . Z e l$. cerealis L. $E_{u t}{ }^{g}$ ) modelta Ind. $O$. americana Gall. Ital. feltiva $A m$. faltuola $L$. Eur. fplendida Tranqb. gloriofa ltal. Ipeciofa L. Eur. cyanea Am. $M$. practiofa Germ. nitida Siam. aurata Penfylv. linubata Angl. ') carnifex Germ. fanguinolenta L. Eur. ") marginata $L . E_{z}$ \% " )

$\mathrm{C}_{2}$

CHRY. bes.

r) Chryfomele à rayes cour.

s) L'Arlequin doré.

t) La Chryfomele bleuc a bordure ronge.

i) Das Blutbabnclben. La Chryf. nnire à bordure rouge. dées. 
Chrysomela. Schach Crioceris. 2 dentata Afro

Germ.

analis $L$. Suze. aucka Germ. barbara $A f r$. marginella' L. Eur. hannoverana.

B) Ranunc. $\mathrm{H}$.

y) Potent. $H$. areata Gall.

5 punctata Germ. Crotonis Am. M. fcutellata Germ. pecioralis Germ. litura Angl. flavicans Germ. lineola Am. facra L. Palaeft. haemorrhoidalis L. Eur.

$\beta)$ viridis.

fucata Ital.

aenea L. Eur. ${ }^{w}$ )

bulcharenf. S. $N$.

2 punctata C. B. S. philadelphica $L$. Armoraciae L. Eur. Cochleariae Germ.

B) virefcens. pallipes Germ. Sophiae Germ. Raphani (nec Galleruca). 122. aeruginea Ital. C R I O CER I S. ( Schnurrkï. fer.)

2 tuberculata Afr.

w) Le petit vertubleu. Porro: Chryf. Baaderi.'P. melanocephala. $N$. Holl. cantharoides Angl. 4 maculata C. B. S. 6 punctata $C . B . S$. variegata Cajen. pygmea Cajen. teftacea $I n d . O$. ochreata Guadal. Betulae Lapp. . fulvicollis Gall. minuta $C$. B.S. erythroceph. C. B.S. 4 puftulata Siam. impreffa Siam. brunnea Germ. retufa Guadel. merdigera Eur. xy Chryfomela $L$. 12 punctata Eur.

Chryfomela $L$. I 4 punctata Germ. ruficollis Cajen. 5 punctata Germ. ebraea Cajen. praeufta. unifafciata N. Holl. 2 fafciata $N$. Holl. nigripes $N$. Holl. grifea $C . B . S$. oculata N. Holl. bioculata C.B.S. pallens. fucata $A m$. $M$.

Crio-

x) Le Criocere, rouge du Lys. 
Crioceris. aulica C. Galrer. ruficollis Angl. B. $S$. cyanella Eur. Chiysomela $L$. violacen $\mathrm{S}, N$. bicolor S. $N$. melanupa Eur. Chryfomela $L$. flavipes (Germ: ${ }^{y}$ ) Cirysomela $L$. funípinofa Eur. rufipes Germ. ") cyanocephala $N$. Holl. Afparagi Eur. Chryjomela. L. violacea Gall. Phellandrii.

Chryfomela $L$. campefris Eur. Chrufomeln $L$. elongata $C . B . S$. trilineata $C . B . S$. equeitris Cajen. vittata Carol. innuba $A m$.

52. 2 maculata Germ.

(nec 4 macul. neque Galleruca.)

G A L L ER V C A. 1 käfer.)

2 maculata $A m$. teftacea C. B. S. falciata Amz.

y) Le Lupere noir à corcelet et plattes rouges.

(Forcht.

z) Le Lupere noir ì pattes rouger. flavicornis Cajen. crenata Cajen. Tranquebar. S. $N$. littoralis Eur. - Cajennenfis. nigripennis Strin. Tanaceti Eutr. Chryfonuela $L$. ruftica Germ. I 2 punctata. Alni Eur. a) Chryfomela $L$. Baftiae (nec Iamaicenfis).

Laufoniae S. $N$. Abfinthii Sibir. concolor Afr. Guadelupenf. picea $A f r$. Betulae.

Cluy fonela $L$. tricolor. fericea S. $N$. livida Germ. Vitellinae Eur.

B) vulgat. $M$. Chry fomela $T$. ruficauda. Avicenniae $A m$. mer. Lactucae Germ. calcarata Germ. punctulata Gern. morbillofa Germ.

C 3

GALLEl'Aune.

a) Le "Gribouri bleu de 
GALLERVCA. cruenta Ind. Or. coccinca Eur. cruciata Germ. quadripultulata Germ. Boviftae Germ. 20 punctata Eir. triloba $C . B . S$. lineola lial. Nympheae Euvr. Ciry fomela $L$. Capreae Equ. Chryjomela $L$. cincta Tranqb. palliata Tramqb.

Cerafi Suec. Chryfomela $L$. livida Seland. mclaunra Suec. calmatienfis Eur.

Ch.y Somela $L$. abdominalis $C . B . S$. Morio C. B.S. Canguinea Gorm. tenella Eur.

Chry fonela $L$. pista Ind. deuticornis Am. mer. nigricornis Gern. Chryfonela halenfis $L$. acrugiuea S. $N$. (nec Ciftela.) thoracica Amr. caroliniana. difcoidea Aw. bor". glabrata $\mathrm{Iam}$. palleus Guadal.
GALLERVCA: a guttato An. bor. 4 guttata Cajenn. equefris Aneric. cincta Lujitan. picta Tranqb. nobilitata Cajenz. quadrifalciata Cajen. albicollis Cajen. 2 finciata Guir. famelica An. bicolor $A m$. Chry Somela $L$. aeftuans Am. mer. Volkmariae $A m$. mer. calida Anv, ner. sequinoctialis $A m$. mer. Chry fonta $L$. oletacea Germ. b)

Chry fonela $L$. Erucae Germ. ${ }^{c}$ ) albicollis $N$. Holl. fulvicollis C. B.S. Napi Gernn. ') . Hyofcyami Germ. ") Chry fonela $T_{\text {. }}$ nigripes Angl. f)

GALLE-

b) Dir Erdfoh. Chryfomele fauteufe potagere. L'Altifie bleu.

c) Der Senfflolskäfer.

d) Der Sicckribenfiolskïfer.

c) Der Bilskrautflobkäfer. L'Altife du Choux.

f) Der fibmarzfiifsige Flob. kïfer. L'Altife noir ovale. 
G A L L E R V A. nitidula Eur.

Helxines. ${ }^{\text {g) }}$

Chryfomela $L$.

fulvicornis SuEc.

3 fafciata. Eur.

Chry fomela $L$.

Modeeri Eur.

Chry fomela $L$.

femiaenea Germ.

erythroceph. Eur.

Chry fomela $L$.

Sifymbrii Germ. atricilla Eur. ${ }^{n}$ )

Chry fomela $L$.

Nafturtii Germ.

dorfalis Angl.

anglica.

4 pufulata Angl. rufipes Evrr. ')

Chry fomeia $L$ :

fufcipes Angl. ruficornis Germ,

Mercurialis Germ.

advena Am.

teftacea Angl. ")

g) Dor Bucbracizenfiobkäfer. Le Plutus. Chryfomole fouteu-' fe vert dorée.

h) Der filuzarzköpfige Flobkäfer. La Paillette.

i) Der zotbfu/sicinte Flobkäfer. L'Altile de la Mauve. .

k) Der rot bgelbe Flobkäfer. L'Altife faure fans ftries, Por. ro hue pertinent :

P.

I) Altica globofa. Germ.
Gallervca: exoleta ${ }^{1}$ )

Chry fomela $L$.

Holfatica.

Chryfonela $L$. tabida Angl. ${ }^{\mathrm{m}}$ ) Jrafficae (Aerm. ") nemorum Eur. ')

Chry fomela $L$. atra Hafn.

Euphorbiae Germ. hacmisphaer. Eur. Chry fomela $L_{\text {. }}$ Suriuamenfis. Chry fomela $L$. S. Littera Surin. 'Chry foniela $L$. elongata $\mathrm{S} . N$.

St. crucis.

1 4. hortorum Anu mer. Erotylus,

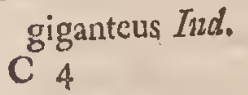

\section{Chry. '}

2) Altica flexuofa. Der bognicbte Flobkäfer. Germ. P.

Germ. P.

3) - orbicularis.

P.

4) - pratenfis,Germ.

5) - Verbaŕci.Germ.

P.

J) Der Diftelflohk. L'Altise fauve à frries.

m) Altica al.der verblicbene Flobkïfer. L'Altife jaune.

n) Altica al, Der Koblfols. käfer.

o) Altica al. Dor Hainfiah. käfer. P. L'Altife à bandes jaunes. 
Chry fomela $L$.

Erotylvs. reticulatus Braf.

6 fafciatus Am. mer.

hiftrio Cajen.

teftaceus Am.

gibbofus Ans. sner.

Chry Jomilu $L$.

notatus Cajen.

concatenatus.

5 punctatus Ann. neer.

Ch:y fomela $L$.

punctatifinus Amo mer.

variegatus Surin.

gemunatus Cajern.

longimanus Ind.

abdominalis Braf.

falciatus,

Zebra Cajent.

alternans.

Surinamenfis.

Coccinella L.

Morio N. Holl.

limbatus Cajen.

cupreus $N$. Holl.

nebulofus Am. mer.

dilatatus C. B. S.

finaragdulus $N$. Holl. amethyftinus $N$. Holl.

bicolor N. Holl.

27. rufipes Germ.

CE⿱R⿴囗十

gigas Gall.

fufeus $C . B, S$,

Cistela, (Fadenkäfor.)

cervina Eur.

Chry fomela $L$.
Cister a. cinerea Eur. livida Terra del $F$. ceramboides Eur. Chry fomela $L$. lepturoides $E u r$. tellacea $B a t b$. picipes Dan. fulphurea Ezwr. Chry fomela $\dot{L}$. bicolor Germ. ruficollis Eur. coerulea Barb. vigripennis Gall. anıalis Tranqb. rufipes Germ. fulvipes Germ. varians Germ. murina Eur. Chry fomela $L$. ferruginea Germ. thoracica Germ. Aavipes.

Evonymi Germ. lumeralis Germ. nigrita Germ. maura Germ. fufeata S. $N$. pallipes Germ. Morio Suec. anguftata Angl. pallida Angl. fentiva C. B.S. aulica $C$. B. $S$. vitrata Cazol. hirta C. B. S. pubefcens Dan. 
Cistela. 35. (exlineata. ${ }^{p}$ ) Cryptocephadvs. laetus ZYGIA.

oblonga Orient.

Zonitis. (Gürtelkäfer.)

Gernt.

Atraphaxidis Sibir.

lunulatus.

praeufta Ital.

pallida Am. mer.

angulata $\operatorname{In} \int$. Am $t$.

mutica Hung.

12 maculatus C. B.S.

obfitus Ans.

lineatus Braf.

tridentatus.

fulvipennis.

chryfomelina Orient.

Ciryfomela $L$.

flava Orient.

roftrata Barbar.

Apalvs:

2 maculatus Eur. Meloe $L$.

4 maculatus Ani. borr.

ALBVRNVS.

\section{groffus Cajen.}

Sagra.

femorata Ind. orient. dentipes $C$. B. S. .

Cryptugerhalvs.(Fallk.)

longipes Gernz.

3 punctatus Ital.

Salicis Germ.

6 notatus Barb.

6 inaculatus 1tal.

4 punchatus Eur.

Chryfomela $L$.

4 notatus Barb.

3 maculatus S. $N$.

P) Purro:

I) Ciftela fufca, P.

2) - opaca. P.

3) - brevis. P.

4) - linearis P.

5) - bipuftulata. P.

taxicornis Ital.

venufus Ans.

Gorteriae C. B.S.

Chryfomela $L$.

8 punclatus Barb.

maxillolus $C . B$. $S$.

auritus Germ.

Chry fomela $L$.

Lentifci $A f r$.

Chrys. variolosa $L$.

didymus $N$. Holl.

4 maculatos Germ.

Chryfoneli $L$.

Havicollis Sibir. Iongimanus Stere.

Chryfonela to.

Lar. An. mer.

marginatus Germ.

pubefcens $A m$. bor.

2 punctatus Eur.

Chryfonela $L$.

lineola E Eur.

2 maculatus Ital.

cordiger Eur.

Chryfonsela $I$.

variabilis $\mathrm{S} . N$.

3 lineatus $5, N$.

C 5

Crypto. 
Cryptocephalvs Bothnicas.

Chryfomela $C$. frenatus Germ. obfcuras Eur. q)

Chryfonsela $L$. luridus S. $N$.

Vitis Eur. plagiocephal. Gall. Coryli Eur.

Chry Somela L. pallens Chin. variegatus It'al. B) immaculat. trifafciatus Chin. ruficollis St. Hel.

B) bipunctat. nigripes $2 m$. 6 punctatus Eur. Chry fonela $L$. flavefcens Germ. coerulans $A f r$. violaccus Germ. cupreus Cnjon. rubrifrons. calcaratus Sier, Leon. lobatus Hung. rufipes C. $B . S$. cyaneus Gall. cinctus Am. mer. bucephalus Germo Seheftedii S. $N$. collaris Sibir. Cericeus Eur. réc.
B) Cr. Hypochaeridis L.

Cryptocephalvs, nitens Eur. Chryfowiala $L$. glabratus Braf. vittatus Eur.

B) quadrum $\mathbf{M}$. limbatus Cajen. nitidulus Rol. Labiatus Eur.

Chry fomela $L$. flavilabris Geivr. flavipes Ital. flavifrons Germ. Hübneri Germ. Moraci Eur. Chry fomela $L$. 8 guttatus Germ. I 5 guttatus Braf. Io punctatus Eur. Chryfomela $L$. hieroglyphic. Ital. coloratus S. $N$. I 2 punctatus Germ. haemorhoid. Gall. 2 puftulatus. 2 falciatus $A f r$. 4 puftulatus Stuec. Chry fomela Lo notatus $A m$. Hiftrio Ital. Sespolinus Am. Chry fomela $L$. Königii Tranqueb. paracenthefis Eur. 
Chivy fomela $L$.

Cryptocejeialvs. Hor. dei Bayb.

concolor Eur.

Pini SizBc.

Chry fonzela $L$.

reticulatus Cajens.

pufillws Germ.

minutus Germ.

parvulus S. $N$.

gracilis Gall.

97. pygmaeus Gall. ${ }^{5}$ )

H I S P A. (Stachelkä-

fer.)
atra Eut. s)
teftacea $A f r$.
hyfrix S. N.
2 puftulata Ital.

fpinipes Surin.

fanguinicollis Am.

ferrata Suriv.

dentata Cajen.

angulata Cajenz.

emarginata Surisu.

ferraticornis Suriz.

bidens Surin.

nigricornis C. B.S.

I4. fulcata Susin.

DRYOPS. (Steinkä̈fr.)

femorata Helv.

aenea $A m$.

livida Otaheite.

r) Porro:

I) Cryptoc variabilis, P.

2) - affinis, 1 .

s) La Chataigue noire.
Dryops, lineata $N . Z$ Z

cyanea $N$. Holl. marginata $A m$ in. mert.

vittata $A m$. rufifrons $A m$. bor.

Tiluvs. (Rouchkinfer.) elongatus Eur.

Chry fonela $L$. damicornis S. $N$. Weberi S. $N$. ambulans Gomn. ferraticornis Gall. Germi.

LAGRIA. (Schinnikiffer.) tuberculata Cajen. villofa $C . B . S$. pubelcens Eur. Clary fonela $L$. hirta Entr.

Clury fonela $L$. abdominalis $17 \mathrm{~L}$. tomentofa $N$. Holl.

B) fufca. obfcura Guin. lugubris Guin. fcutellaria Hifp. atra Eur. Dermeftes hirtzis $L_{\text {s. }}$ nigra Eur. lineata Braf. flavipes Germ. coerulea Err. metallica Barb. nigricornis Sute. miinuta $N . Z \ell$. 18. $\beta$ ) virefcens. 
Cerocoma. (Kronenkï. MYI A B IS. praeufta

$$
\text { fer.) }
$$

Schaefferi Eur.

Meloe $L$.

Vablii Barb.

Schreberi Eur.

microcephala $A f r$.

lineata $C . B . S$.

ruficollis.

Lrтta. (Reizhïfer.)

veficatoria Eur. ${ }^{*}$ )

Meloe $L$.

fegetum Barb.

ß) cyanea.

nitidula $C . B . S$.

collaris RoJf. .

gigas Guin.

Syriaca Eur.

Meloe L.

ruficollis Ind. or.

teftacea Tranqb.

feftiva Sibir.

marginata $C . B . S$.

vittata Am. .

atrata Barb.

erythroceph. Germ.

oculata Guin.

dubia Sibir.

afra.

Meloe $L$.

17. haemorrhoid. C. B. S. M х А в к I s. (Fliegenkïfer.)

falciata $F$ ind.

Cichorci $O$.ient. Miloe $L$.

t) (antharide.
Barl.

narginata Barb.

algirica.

capenfis.

Hermanniae Guin. punctum Tranqb.

Io punctata Ital.

Meloe 4 punctata L.

4 punctata Ro/.

3 maculata Orient.

12. argentata Seneg.

Horia.

maculata Cajen.

teftaces Tranqb.

L Y M k XXL ON. (Holaboh$r e r$.

dermeftoides Eur.

Cantharis dermeftoides L.

abbreviatum Sierra Leost. probolcideum Germ. barbatum Germ. ruficolle S. $N$. navale. ")

Cantharis $L$. flavipes Germ. Morio Germn. ")

CvCvivs. (Rindenkäfur.)

depreffus Germ.

Cans.

u) Der Scbiffwerfikäfer.

v) Auplius :

I) Lymexylon fabellicorne, $\mathrm{P}$.

2) - laevigatum, $P$. 
Cantharis fanguinolen- LAMPYRIs. phofphorea $L$. ta $L$.

Cvevivs. fulcatus Croat. rufipes Barb. coeruleus Germ.

Tenebrio depreffus $L$. feftivus Germ. caftaneus Germ. dubius Am. bor. flavipes Eur.

Cerambyx planatus $L$. pallens Germ.

dermeftoides Germ. An. m.

teftaceus. muticus Germ.

13. monilis Germ. ")

Cossyphys. depreffus Ind.

LAMPYRIs. (Leuchthäfer.) noctiluca Eur. ${ }^{x}$ ) fplendidu'a Eur. corrufea Am. bor. obfcura Terrad. Fuego. laticornis Seneg. pyralis $L$. Am. mer. cincta Poulicandor. marginata $L$. Am. pectinata $A m$. flabilicornis Braf: Hefpera $A m$. ignita L. Am. mer. lucida L. Am. mer. nitidula $A f r$. latus.

w) Amplius: Cuc, bipuftu. worm.

$x)$ Lé Ver luifant. Glow.

Iaponica.

atra Am. bor.

capenfis. .

vefpertina $\mathrm{S}$. $N$.

auftralis $N$. Holl.

italica $L$.

vittata Guadel.

verficolor S. $N$.

maculata S. $N$

mauritanica.

26. hemiptera Gall.

OM ALYSYS. (Glanzkä-

$$
\text { fer.) }
$$

futuralis Gall.

Рукоснвол. (Fenerkifer.)

coccinea Eur. ${ }^{y}$ )

Lampyris $L$.

rubens Gall.

flabellata Anr.

pectinicornis Germ.

Cantharis $L$.

ro maculata Cajer.

Lxcvs. (Brandkäfor.)

latilimus Sierr. Leor.

Lampyris $L$.

palliatus C. B.S.

roftratus $A f r$.

Lampyris $L$. probofcidens $A f r$.

ferraticornis $N$. Holl. praeuflus Tranqb. fafciatus Cajen. reticulatus $A m b$ bor.

E.xCV\&. 
Lyovs. bicolor Afr.

Lampyris $L$.

fanguineus Eus:

Lampyris $L$.

Anrora Germ.

vigripes Siant.

minutus Norveg.

flabellicotnis Cajern.

15. ruficollis $C$. $3 . \dot{S}$.

RIPI PHOKVS. (Kammkit-

$$
\text { fer.) }
$$

fubdipterus Gall.

ferrugineus Ind. $O$.

nafutus Iopon.

fpinofus $\mathrm{S} . N$.

pectinatus Aner.

paradoxus Eur.

flabellatus 1tal.

punctatus Cajens.

6 maculatus Am.

lituratus S. $N$.

2 maculatus Hung.

dimidiattis.

limbatus.

14. populneus Girm. ${ }^{2}$ )

MORDELIA. (Stachelkï-

\begin{tabular}{|c|}
\hline $\begin{array}{l}\text { aculeata } L . " \text { ") } \\
\text { fafciata Ezr: } \\
8 \text { punctata Am. } \\
\text { teftacea } A f r \text {. } \\
\text { a maculata. } \\
\text { ventralis Germ. } \\
\text { abdominalis Germ. }\end{array}$ \\
\hline $\begin{array}{l}\text { Amplius : Ripiph. carirtu } \\
\text { s. } \\
\text { La Mordella à tarrière. }\end{array}$ \\
\hline
\end{tabular}

MORDELIA, humeralis Eur. lateralis Gerin. frontalis $L$. Enur. atra $D_{\text {ann. }}$ thoracica L. Eur. ruficollis Gall.

14 Hava Eurr.

Donacia. (F/ufspflanzerzo kiffer.)

craftipes.

Leptura aquatica $L$.

Equileti S. $N$.

Feflucae Gerni.

dentipes Gerin.

Nympheae.

Sagittariae Gq*n.

nigra Germ.

clavipes Germu.

falciculata Cojen.

fimplex Eur.

11. Hydrocharis, ${ }^{b}$ )

TRICHIVs. (Schirmblumen.

käfer.)

Eremita Germb. ")

Sca.

b) Species novac:

3) Don.appendiculata.P.

2) - limibata $P$.

3) - fenicuprea. P.

4) - terfata. $P$.

j) - palunris. $\mathrm{H}$.

6) - vittáta, P.

7) - micans. P.

8) - collaris. P.

9) - difcolor. P.

10) - Striata. $\mathrm{H}$.

c) Scarabé de cuir noir. 
Scavabaeus $L$.

Trichivs. nobilis.

Scarabaeus L.

8 punctatus.

Scarabanus variabilis $L$.

falciatus Eur.

Scarabaeus L.

fuccinctus Gerut.

indus.

Scarabaens $L$.

2 punctatus.

4 guttatus Chiss.

bibens $A m$.

liemipterus Eur.

Scarabaetus $L$.

canaliculatus Am. Sept.

lunulatus Carol.

viridulus Ind.

piger Maryl.

Delta Am.

lineatus $C . B . S$.

nigripes $C . B . S$.

I8. maculatus C. B.S.

Cetonia, (Goldkäfer. Ce. toine.)

goliata $A f r$.

Scarabaeus $L$,

Cacicus Am. $m$.

bifrons $A m$. $m$.

Polyphemus Afr.

micans $A f r$.

chinenfis.

nigrita Ceyl.

aurata Eur. "). Chafer.
Scturabaeus $L$.

Cetonia. faftuofa tiur. marmorata Germ. viridis Ital. metallica Ital. pubefcens C. B.S. opaca $A f r$.

Morio Eur: cufpidata C. B. S. cordata $C . B . S$. 4 punclata Ital. rufipes Ind. holofericea Sturin. nrigofa C. B.S. nitida Am.m.

Scarabaeus $L$. lobata Am.

Carmelita Afr. fulcata Braf.

Chryfis Amn: auftr.

Scarabaezis $L$. lucida Guadel. capucina Ind.

Lanius Am. mer.

Graculus.

Carnifex Am. mer.

Scarabacus Lanius $L$.

rauca $C . B . S$.

cornuta C.B.S.

triftis $A m$.

fmaragdula $A m$. falcicularis $A f r$.

Scarabaens L.

aulica C. B.S.

purpurafcens Ind. $O$ :

capenlis. 
Scarabaezus $L$.

Cetonia. fignata $C . B . S$.

marginata Guin.

ornata Guin.

marginella Sierra Leom. lineola sist. Mer.

Scarabaews $L$.

ß) Ephippium $\mathrm{M}$.

y) Scar. 'Surinamenfis L.?

friata Guadel.

finuata $C . B$. S.

fafciata Alexands.

thoracica Alex.

olivacea Sierra Leon.

interrupta Seneg.

picta $O r$.

trilineata C. B.S.

elata Guin.

femipunctata.

5 lineata.

atromaculata Tranqb.

limbata Aegypt.

fplendida Ind.

elegans Corom.

4 maculata $A f$.

cuprea Surin.

africana.

futuralis $A f r$.

vitrata $A f r$.

fulgida.

Iris Surin.

gagates Sierra Leor.

tetradactyla $A m$. $m$.

Scarabaeus $L$.

clavata Am. mer.

fucata Am. mer.
Cetonia. lurida Braf: aurichalcea Ind. nitidula Afr. philippenfis. nolata $N$. Holl. feftiva Tranqb. abbreviata Seneg. maculata Corom. acuminata $C . B . S$. haemorrhoid. C. B.S. areata Virg. hirta Eur. ${ }^{\circ}$ )

Scarabaeus $L$. funefta-Ital. ftictica Germ. ') 'Scarabaeus L. punctulata Seneg. floralis $A f r$. I 4 maculata. verficolor Aegypt. variegata Tranqb. aequinoctialis Senzeg. fanguinolenta Seneg. difcoidea Beng. lugubris C. B.S. Hiftrio Aegypt. modefta Tranqb. gloriofa St. Domingu. hottentotta $C . B . S$. 99. cruenta.

MELOLONTHA. (May$k$ käfer.)

Melo.

e) Der raucbe Goldk. C. velu, le Dos velu.

f) Der Punktkäfer. Le Drap mortuaire. 
Melolonatha. Migma A. Fullo Eur. ${ }^{\text {E) }}$ )

B) M. alba. Oliv.?

y) Scar, albus Pall.?

ס) Scar. helolcucos $P$. ferrata Corom.

vulgaris Eur. ")

Scarabaeus MelolonthaLii

villofa Eur.

Scar. Sappicola $L$.

pilofa Hung.

occidentalis Eur.

Scarabaets $L$.

fervida Am. Sept.

reflexa $A f r$.

Alopex C. B. $S$. tomentufa Ind. $O$.

folftitialis Eur. ${ }^{i}$ )

Scarabaeus L.

candida Ind. $O$,

atra Enr.

B) fufca $O t$

aequinoctialis Hang:

Pini Barb.

2 maculata Chin.

Atriplicis $B \omega w b$.

oblongata Germ:

ruficorms Germ:

lanigera Ind.

Scarabaeus I.

longicornis C. B.S:

punctata $A m$.

g) Le Fonlun.

h) Le Hannetón. Inin.
Scrirabaeus $L$.

MELOLONTHA. viridis

C. B.S.

aenea $N$. Holl.

futuralis $N . Z e l$. bicolor C. B. S. barbata Ind.

elata Tranqb. dorfalis Tranqb. glacialis Terr. d. F. Jurida.

Ariata Terr: d. E. triftis Am. bor. teflacea Terr. d. Fi: hirticollis Afr. pallida $C . B . S$. ruficollis Coron. variolofa $C . B . S$ : fignata Iamaic.

marginata.

cosruleoceph:

brunnea Ear:

Scarabaeess $\dot{L}$. melanoceph. Braf: érythroceph. Cosom: mixta $A f r$. obfcura Afr. rufa $C . R . S$. feftiva $N$. Zel: laeta $N$. Zel. holofericea Rofj:

Iulii Germ. nigrita $A m$. Frifluii Germ:

B) aenea. D y) cyanea.

Melo. 
MELOLONTHA. Vitis Eur.

Cardui Afr.

Serratulae Barb. faftuofa $I n d .0$.

errans Angl.

umbrola Guin. rupicula $C . B$. $S$. iunulsa. elongata. rauca Corom. ferruginea Corom. fplendida C.B.S. aulica $A f r$. lucicola S. $N$. atrata S. $N$. arbicola Am. horticola Eur. ")

Scarabaezs $L$. abdominalis Ital. foricola Afr.

B) elytr. atris. arvicola Roff: praticola Sib. fruticola Entr.

ß) Sc. fegetum $\mathrm{H}$.

y) Sc. auftriacus $\mathrm{H}$. agricola Germ. Scarabaerus L. ruricola Angl. atomaria C. B.S. farinola $E$ ur. ') Scarabaeus $L$. vert.

k) Hanneton à corcelet

1) L'ćcailleux violet.
Melolont.fquamofaGerm. regia $A f r$. Scarabaens aulicus $L$. argenten Angl. graminicola Germ. chryfomelina Auft. cincta Guadel. probofcidea Ind. fpinipes C.B.S. deutipes $C . B . S$. podagrica Corom. arthritica C. B.S. gonagra C.B.S. craflipes $C . B . S$. cancroides $C$. B. $S$. marginella $C . B . S$. pulverulenta Germ. Morio Barb. fylvicola N. Holl. fublpinofa Am. mer. longipes. abbreviata. C. B.S. minuta C. R.S. mutabilis Tranqb. variabilis Eur. Am.

B) aurulenta. verficolor Sierra Leotz. picea C.B.S. araneoides C. B.S. lineata Sierra Leon. gibba C. R.S. monticola $N$. Holl. humeralis Eur. Vrfus C. B.S.

$\beta)$ nigripes. Lynx C. B.S. 
Melolontha. crinita C. BVprestis. rutilans B. $S$.

Scarabaeus L. .

hirta Sib.

Vulpes Sib.

Meles Barb.

Bombylius $A f r$.

I I 9. vittata Ind.

Burrestis. (Prachtkäfer.

\section{Le Richord.)}

unidentata.

bicolor Am.

4 dentata.

gigantea $A m$.

vittata Ind.

fafuofa Am. Sept.

regalis Cajerr.

collaris Cajen.

attenuata Rio Ianeiro.

punctatifima Sierr.

Leon.

rufipes Maryl.

berolinenfis.

corrufca Iam.

micans Ital.

decora Am.

funefta Guin.

umbrofa Guin.

Jurida Am. bor.

obfcura Am. bor.

punctata Barb.

mórbillofa Am. neer.

aurifer Cajen.

fafciata Am. bor.

lucidula Surin.

rauca Barb.

Atriata Penfylv.

Gern. ${ }^{\mathrm{m}}$ )

ignita Ind. O.

lineata $A m$.

ocellata Ind. $O$.

aenea Corom.

maculata Sib.

flavomacul. Suec.

interrupta Seneg.

Aernicornis Ind. $O$.

Chryfis Ind. O. " )

$B$. ftenicornis $L$.

caftanea Sinzeg.

violacea Sierra $L$.

equeftris Braf:

depreffa Orient.

Mariana L. N. Holl.

farinofa $N$. Holl.

ventricofa Mallog.

finaragdula Ind.O.

fulminans $T$ ind.

cyanipes Amomer.

modefta Braf.

blanda Am. m.

aurata $A m$.

fulgida $I n d . O$.

3 punchata Am. mer.

elegans $C . B . S$.

3 fafciata Am. ner.

9 maculata Eur.

porcata Orient.

dorfata Anu. mer.

chryfoltigma L. Eurr.

D 2

BVPRE:

m) Der goldgeränderte $P$.

i1) Buprefte à pointe, The thitk Gogle. Betle. 
BVPRETIS. imprefla lnd. affinis Germ. fróntalis Curol. ornata Penfylu. fercata Guin. tacuiata Enur. clongata Barb. pulcra Marocco. gibbofa C. B.S. nabilis Cajen. cylindrica Orient. Volvulus Amo mer. fcabra. Ind. Trochilus Auft. decoftigma Grrm. ${ }^{\circ}$ ) (uec chryloliigma). 8 guttata L. Eur. falcicularis $L . C . B . S$. variolaris $R$ Rof? Onoperdi Barb. hirta L. Afr. auftriaca. fibirica. . fplendens Chir. fufca C. B.S. ruftica L. Eur. canaliculats Afr. reciculata A. 7tal. acuminata $R o /$ : excavata Tranqb. moefta Germ. lugubris Eur. B) plebeia $\mathbf{M}$. cariofá Roff.

o) Der P. mit .2. gelben Punkten.

BVPRESTIS. undata

\section{Germ.}

tenebricola Gall. mer. Tenebrionis L. Eur. Afr.

Rubi L. Eur. aurulenta Girm. terfata Marocco. cyanicornis Gall. $M$. 2 inaculata L. Ind. 4 maculata $I n d$. triftis $L$. Ind. cuprea $A m$. tarda Germ. . metallica Gall. mer. nobilitata $L$. Ind. appendiculata $G$ crm. bicolor Barb. umbellatarum Barb. Morio Hafn.

4 punctata $L$ Eur. cruciata $N$. Holl. lineata Karb. manca Germ. ${ }^{\text {p) }}$ pygmaea Barb. minuta L. Eur. meditabunda Am. bor. atomaria Surin. viridis L. Eur. 2 guttata Angl. linearis Dan. atra Germ. elata Gern.

BVPRES-

p) Le Richard rubis. Porro: I) B. Puni P. 
BvPRESTis. pavida. emarginata Cioll. ruficollis $A m$. felliva $A f r$. nitidula L. Eur. nitens (lucidula A.)

Germ. lacta Girm. Salicis Eur. Sophorae Guin. difcoidea Barb. cyanea Germ.

132. candens A. Germ.

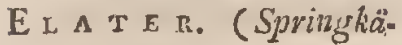
fer.)

flabellicornis $I_{\text {. }}$ Ind. fafcicularis $A m$. fpecinfus Ind. luridus Corom. foveatus Guirz. oculatus L. Amu. . lufcus $A m$. coecus $A f r$. lineatus Braf. noctilueus $L$. Am. m. pholphoreus $A m$. ignitus Cajen. fulcipes lnd. $O$. 4 maculatus $A f r$. rubiginofus Sievr. $L$. ligneus $L$. An. $m$. rufus Germ. ferrugineus L. Eur. q) porcatus Am. mer. fuicatus Am. mer.

१) Le Tauptin rouge.
EL A T E R. virens $A m$. IIIS. fulgeus Ind. inaequalis Ital. aterrimus L. Eatr. niger L. Eur. murinus L. Eur. h.lofericens Germ. teflellatus L. Eur. atomarius S. $N$. fafciatus L. Eur. varius Gall. aeneus 1.. Eur. B) germanus $L$. impiefTus Steec. pectinicornis L. Eur. furcatus $I n d$. futuralis Seneg. vittatus Germ. cupreus Angl. cruciatus L. Eur. fyriacus L. Or. caftaneus $L$. Eur. livens $L$. Ersr. mefomèlus $L$ Eur. denticollis A. Germ. linearis $L$. Eur.

B) margincllus $M$. obfcurus L.Evr. flavipes $A m$. callanipes (nec caftane. us) Gaudel.

maculstus Am. mer. filiformis Ital. triftis L. Eur. marginatus L. Eur. D' 3 ELATER. 
EL A T E R. thoracicus Angl. E c A T E R. 3 maculatus

Gall. r)

ruficollis $L$. Ear.

brumeus $L$. Eur.

haenatodes Gcrm.

fanguineus Germ. s)

B) maior.

ephippium Germ.

praeuftus Germ.

teftaceus Germ.

balteatus $L$. Eur.

elongatulus $A u f l$.

lateralis A. Germ.

melanocephal. Corom.

fputator L. Eur.

analis Germ.

elegans Am, mer.

variabilis Germ.

pilofus Germ.

longicollis Gall.

pallipes Tranqb.

Ariatus Suec.

rufipes Germ.

minutus L. Ear.

pallens Gall.

limbatus Germ.

riparius $L$ app.

advena $H i j p$.

nitidulus Cajen.

puichellus $L$. Eur.

2 gettatus Gall. mer.

equeflris $\mathrm{S}$. $N$.

2 macularus Gall. let rouge.

r) Le Taupin noir à corce-

s) Le Taupin à etuis rou-
Germ. 4 inaculatus Ital. politus Dalikarl. 6 guttatus C. B. S. bupreftoides Germ. pygnaeus Germ. notatus Corom. feltivus Aegypt. 2 puftulatus L. Germ. 92. 4 puftulatus Germ. ') LvCANvs. (Schröter.)

Alces Afiae.

Giraffa A. Afine.

Cervus L. Eur. ")

Elaphus Virgin.

Saiga A. Am.

Capreolus L. Germ.

Dama Virg.

femoratus Cajen.

Bifon An. mer.

Gazella Siam.

Lama Ind. Or. futuralis. paralelepipid. L. Eur. v) cancroides Terra Diem. LvcA-

t) Porro:

I) Elater bicolor, P.

2) - cinctus, $P$.

3) - rubens, $P$.

4) - trifaficiatus. P. P.

5) - Canguiniscollis.

i) Le grand Cerf volant. Stag Flying Fly.

v) Le cérf -vclaut paralJelipipede. La petite Biche. . 
L V C A N v s. tenebroides Roff.

16. caraboides L. Eur.

B) virefcens ${ }^{w}$ )

Passalvs.

interruptus $A m$. mer. Lucanus $L$. dentatus Guadel. minutus Ans. mar. PRIonvs. (Forftkäfer.) longimanus Am. mer. Ceranbyx $L$. fuliginofus Ams. roftratus Siam. lineatus Am. Cerambyx $L$. nitidus Braf. Faber Eur.

Cerambyx $L$. fcabricornis Eur. deplarius Suet. Cernnibyx $L$. nucronatus $A m$. arcuatus Terr. Diem. maculatus Seneg. cervicornis Ani.

Cerambyx $L$. fpinicornis. buphthalmus Ind. Or. coriarius Eur. ${ }^{x}$ )

B) Cer, inbricornis L. giganteus Cajen. Curambyx $L$.

w) Lute. fearabaeoides P. x) Der Gerber: Le Prione. Le Capricorne Prione.
PR I $O$ N v s.cylindricus $A w$. bor. armillatus Ind. Cerambyx $L$. Luzonum. ferripes $A f r$. danicornis $A n$. 2 fafciatus $A m$. Cerambyx $L_{\text {. }}$. Thomae $[n] . T \%$. 2 lineatus $A m$. fpinibarbis Ant. mer. Cerambyx $L$. palmatus Guin. maxillofus $A m$. canaliculatus Am. Inf. cinnamomeus An. mer. Crrambyx $L$. melanopus Am. mer. Cerambyx L. fpinolus Tranqb. barbatus Am. nuer. 33. pectinicornis Seneg. ${ }^{\text {Y) }}$ CERAMBXX. (Bockkïfer.) mo(chatus L. Eur. ") " virens $L$.

ß) longicornis

y) dentipes nitens : $A f$ : aeq. afer. feflivus $A f r$.

D 4

Ceram-

y) Porro: Prionus Eerrarius. $P$.

z) C. odoriferant. Musk. beetle. 
CER A M BYX. vittatus Bral.

velutinus $A m$. fericeus Braf. futuralis Am. $m$. elegans Am. $m$. latipes C. B. S. longipes C. B. S: interruptus.

Cerdo L. Eur: a)

Heros Euy.

fpinicornis Sierra Lean. ater $C . R . S$.

Batus L. Am. sn. ferrugineus $L$. Ind. $O$.

B) gigas $\mathbf{M}$. alpinus /. Eur. h) fealaris Am. $n$. ebulinus $C$. B B.S. Moria Cajeqz. Kaehleri L.'Eur. Lundii Trangb. Desfontainii Barb. humeralis S. N. fuccinctus $L$. Am: friatus Cajen. rufipes Am. $m$. dimidiatus Cajen. bicolor Cajerr. deprefliss Am. Inf: fafciatus Tranqb. barbicornis L. Iind. $O$. nebulofus $L_{0 .}{ }^{\mathrm{c}}$ )

a) C. favetier.

b) La Rofalie.

c) Der. Tamienbuck. Le Ca. pricorne noir nuarbré de gris.
CERAM В $\mathrm{X}$. obfeurus C. B. S. grifeus Germ. collatus Germ. fafciculatus Germ. hifpidus L. Eur. ${ }^{\text {d) }}$ pilofus Germ. balteatus L. Tuffit. rugicollis Tranqb. 2 maculatus Ind. $O$. fplendidus Tranqb. longiconis Corom. marginalis $C . B . S$. brevicornis Sierr. Leon. juvencus $L$. Am. holofericeus Ind. $O$. cinereus Tranqb. 53. longicollis Ind. $O$. LAM1A. (Zauberkiffer.) gigas Guin.

Tribulus $A f r$.

fronticornis $C . B . S$. pedicornis $N$. Eoll. Hyfrix C. $B . S$. crifta $N$. Zel. ambulator Inf: Luzon. textor Eair. ${ }^{\circ}$ )

Cerambyx $L$. vaginator Ind. 0 . crucifera. pulchra Afr. regalis Afr.

LAMIIA,

d) Der Bürflenbock. Le Capricorne à etuis dentelćes.

c) Lé Caprieorne noir cha. griné. 
LAmIA. undata. irrorata $I n d .0$. nigricornis Coroms. aedilis Eur. ${ }^{\text {f) }}$

Cerambyx $L$. atomaria L. Gemm. varia Eur. araneiformis $A m$. $m$ : punctata Cajen. nodola Maryl. cancriformis Iamaic. tuberculata Iam. hebraea Am. m. horrida Cajen. Scorpio Am. m. nixta $S$. $N$. obfeurata S: $N$ glauca $A m$.

Cerambyx. L. bidentata $A m$. fcabra.

praemorla Ġuadel. fpinifera $A m$. $m$. grifator Tranqb. aedificator Ind. $O$. amputator Am. Inv: fternutator Barb. fpinator C. B. S. guttator ifr. nebulofa Gern. repandator Cajen. variolator $I n d$. futor'Eur. ')

f) Le Capric. charpentier.

g) Capricorne noir à ato; mes blancheatres.
Cerambyx $L$.

LAmiA. fartor Gerrm. 'dentator Carol. reticulator $I n d .0$. fcalator Louif. molator Cajen. titillator Carol. vittator Sin. Campech: oculator $C . B . S$. capenfis.

Cerambyx. $L$. hottentotta $C . B . S$. ferrugator C. B. S. humeralis. 5 fafciata Guin. 3 fafciata Sierra Leon. 2 falciata Vamaic. nobilis Cajen. aethiops C. B:S. B) impunctata. variegator Afr. curculionoid. Eur. Corambyx. $L$. lufca Siam. notata Cajesn. lugubris Ital. triftis Gall.

Cerambux $L$. funefla Gall. punctator Chin. crifpa C. B.S. falciculata Cajen. rotator $1 n d$. Glycyrrhiza Sibir. cruciata Rolf: fnliginator Eur.

D 5 Ce. 
Cerambyx L.-

L A M I A. cineraria Rols:

carinata Sibir.

Coquus Am. bor. rufpator $A f$.

tornator Am. bor.

Bankii C. B.S.

faltator.

Hiftrio Tranqb. teflator Afr. pedeflis Eur.

Cerambyx $L$.

rufipes Hung.

Morio Germ.

lineata Germ. .

molitor Ind.

tuberculator C. B.S.

nutator Othahaite.

Rubus Ind.

Ceraminyx $T_{\text {. }}$

8 maculata Tranqb.

fpinicornis Afr.

fabrator Ind: $O$.

Spengleri $A m, m$.

fufcator Tranqb.

bidens $N$ Holl.

Cantor Chin.

Solandri N. Holl.

cornutor Am.

ror. Jeprofa Am.

STENocorvs. (Zahnbock.)

cyaneus Ind.

Lanned Ear.

Cerambyx $L$. gargautcus Maryl. fortivus $A m$.
Cerambyx $L$.

STENOCORvS. marylandi-

cus.

fpinicornis $A m$.

bidens Am. m.

femipundatus N. Holl.

irroratus Amrr.

Cerambyx $L$.

glabratus Am. m.

farinofus.

Cerambyx $L$.

6 maculatus Cajers.

5 maculatus Guvadel.

4 maculatus Am. m.

Cerawbyx $L$. macalofus $A m$. $n$. geminatus Sierra Leorr. cylindricollis S. $N$. pallens Am. in. frepens Galloprov. fafciatus $A f r$. circumflexis Cajen. nanus. annulatus.

10 maculatus Am. lineola Braf. varius Cajens. pubefcens Cajen. villofus Carol. Drurii Am. undatus $A m$. 31. ruflicus $I n d . O^{\text {h }}$ ) Calopus (Holzbock.) Calo:

h) Porro: Stenocorus dis. par, P. 
CALOFVS. ferraticornis Eur. ${ }^{\mathrm{i}}$ )

Ceranbyx $L$.

Ringivm. (Zangenbock.)

mordax Germ. inquifițor Eur.

Cerambyx $L$. indagator Germ. curfor Eur.

Cerambyx $L$. cinctum Germ. Noctis Eur.

Cerambyx $L$. bifalcintum Eur. clathratum $G$ erm. ornatum $P_{\text {enfylv. }}$ minutun Garm. muticum Suec. S A P E R D A. (Schneckenkäfer.)

Carcharias Eur. ${ }^{\text {) }}$

Cerambyx $L$. fcalaris Eur.

Cerambyx $L$. candida. detrita Barb. irrorata Afr. modefta Afr. atricornis Chis. oculata Eur.

Ceranbyx $L$. emarginata S. $N$. tricolor Ind. Or.

i) Capricorne à antenncs denteleés.

k) Le Capricorne ponctne.
SAPERDA. villofa (nec hir-

ta) N. Zeland. unicolor $\ln \int . A m f$. lincaris Eur.

Cerambyx $L$. nigripes Hung. cylindrica.

Cerambyx $L$. 3 punctata Virg. melanoceph. Afr. bilicornis $A m$. mer. barbicornis. difcuidea S. $N$. erythrocephal. Germ. ruficollis Virg. fafciata Sibir. clavicornis $C . B .5$. latipes $C . B$. $S$. vittata $A m$.

Cerambiyx trilineatus L.

6 punctata $C . B . S$. lateralis $A m$. bor. lyncea $N . Z e l$. grilea $N$. Zel. analis $A f r$.

2 dentata Guin:

Cardui (nec nigricornis) Eur.

Cerambyx $L$.

nigricornis (nec futuralis) Germ.

futuralis (nec anuulata) Afr.

annulata (nec lineata). An, mer: 
Saperda. lineata (nec trifis) Tranqb. triftis $\mathbf{A}$. populnea $A m$. Cerambyx $L$. tremula Germ. : punctata Eur.

Cerambyx $L$. virefceny Ital. longicornis Afr. femorata. haemorthoidalis $\mathbf{A}$. Volvulus Cajen. ferruginea Germ.

Cerantyx contharinus $L$.

brunnea Germ. teftasea Grom. violacea Ital. praeufta Eur. Leplara praeufta $L$.

Ephippium Hung. picea Am. Sipt.

hirta Ital.

fcutellata Gern. 56 lineola Ital. CAL L I D I M. (Widder. liäfer.)

obfcurum Terr. Dien. Bajulus Eirr. ${ }^{i}$ )

Cerambyx L.

fenuicum.

Cerambyx $L$. ruficolle Ital. ebulinums $A f r$.

1) L.e Capricorne ì queue.
Cerambyx $L$.

CAL L I D I V M. aeneum Ind. clavipes Gevm. fpinofum Hung. violaceum Eur.

Cerambyx $L$. femoratum Germ. Cerambyx $L$. fulcratum Gurm. amethytinum An. bos. 2 fafciatum Am. mer. acuminatum $C, B . S$. rullicum.

variabile Eur.

Ceranbyx $L$. fariceum Barb. tẹnebrofum Cajes. ruflicum Fur. vittatum Guin. agrefe Germ. Stigna $A m$.

Ceraming $L$. fugax Grulo Prov. pyginaeuin Ital. Angl. equefre Cajen. fulvicolie Surin. hirtum C. B.S. pubeficens $C$. $B . S$. barbatum Trangb. grifeum $B a w b$. compreftum Sian. variegatuin $N . Z$ sl. lineatum $N$. Holl. fulcatum $N . Z_{\ell l}$. fanguineum. Eur: 
Cerambyx $L$.

CALlidivm. tefaceuin Eirr.

Ceramby.x $L$.

pilicorne Ans, mzer.

pracuftum ital.

ligneum.

Alavipes $C . B . S$.

luridum Eur.

Cerambijx $L$.

fufcum Germ.

4 maculatum Gucidal.

trifte Germ.

raucum $A f r$.

hafnienfe.

Cerambyx liciatus $L$. rufipes Germ.

ftrialum Eur.

Cerambyx $L$.

aulicumr S. $N$.

bicolor Ams. mer:

lynceun C. B.S.

Salicis Grerm.

cyaneum Ital. pufillum Germ.

2 maculatum C. B.S.

flavum $A m$.

undatum Eur:

Cerambyx $L$.

terminars S. $N$. colonum Carol. varium $A m$. bor. flexuofum $A n$. minutum $N . Z \&$. thorale Ital. fulminans Am. bor. Cemipunctata S. $N$. atomarium Germ. .
Caleinivm. lufcum S. $N$. arcuatum $E_{n} u r .{ }^{\mathrm{m}}$ )

Leptura $L$ : B) Lunat. $M$. arietis Eur. ") $L_{p} p t: a s a L$. plebejum Eur. Gazella Germ. Leptura ruftica $L$. mallilienle. Liptura $L$. mucronatum $A m$. angulatum. erythrocephalum Ans. detritum Eatr. Leptura $L$. glaucum Ind. Or:

6 fafciatum.

3 fafciatum Lufit. Verbafci Eur. Leptura $L$. ornatum Germ. 4 pundatum Gall. annulare Sianz. aegyptiacum $O r$. myficum Eur. ${ }^{\circ}$ ) Leptura $L$. virens Barb. gibbofum Ital. unifafciatum Gall. picipes:

Cálit.

m) La Lepture aur croiffans dorés.

11) La Lepture à troîs barsdes dorés.

o) Le arlequiné. 


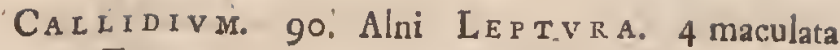

Evr.

Leptriva $L$.

LEPTVKA. (Schnalbock.) unipunctata Germ. haftata Eur. P) 2 punctata Sibir. tomentola Gall. melanura L. Eur. laevis Germ. livida Germ. Arigilata Suec. emarginata Cajen. fanguinolenta L. Eur. meridiana Eur.

Cerambyx $L$. villica Angl. rubra L. Eutr. teftacea L. Eur. pubelcens Suec. virens $L$. Eur. finaragdula Suec. atra Eiur.

humeralis Germ. exclamationis Suec. futuralis Grmm. fcutellata Ital. Jurida Germ. femorata Germ. ruficornis Ital. marginata Norveg. nigra L. Eur. praeufla Germ. 4 guttata Germ. rolirata $A f r$. Iuteiconis Carol.

p) L.e fencore bedeau.
Germ.

4 puftulata Stuec. 8 maculata L. Eur. interrogation. L. Suec. marginella Ital. 9 maculata L. Eur. 7 punctata Hung. I 2 maculata Sibir. attenuata $L$. Eur. calcarata Germ. fubfpinofa Germ. finuata Germ. 4 fafciata L. Eur. auruleuta Germ. dubia Sibir. 6 guttata Germ. 3 falciata Suec. reticulata A. Ital. fericea $L$. Eur. Morio Suec. collaris Etw.

53. virginea L. Eur. 9).

NEC × D A L I S. (Fiegenkïfer.)

thalallina Gern. viridiflima Eur. Cantharis L. coerulefceus Eur.

Cantharis $L$. virefcens Germ.

Cantharis $L$. cyanea Germ.

NECY.

4) Porro:

. 1) Leptura fignata. P. 2) - areuats. P. 
NeCYDALIs. fanguinicollis SYNODENDRON. cylindri.

Gern.

barbara Barb.

felodonia Germ. ,

ruficollis Ital.

melanocephala A. Ital.

triftis Turr. Diems.

atra Eur.

humeralis Angl.

uftulata Germ.

melanura Hifp.

notata Germ.

fulvicollis Germ.

rufa L. Eur.

praeuita Barb.

coerulea L. Or. Eur.

Podagrariae L. Eur.

bicolor $C . B . S$.

clavipes (nec flavipes)

Germ.

tefacea Germ.

glaucelcens L. Eur.

fimplex Eur.

27. Quercus $D_{a n \text {. }}$

Molokchys.

abbreviatus Eur.

Necydalis maj. L. variegatus $N$. Holl.

dimidiatus Eur.

Necydalis mizt. $L$. umbellatarum.

Necystalis $Z$.

SPONDYIIS.

bupreftoides Eur.

Attelabus $L$.

SYNODFNDRON. (Baummager.) cum Eur.

Senrabaeus $L$.

muricatum Eur.

Durmeftes $L$.

capucinum Coron.

dominicum Am. mer.

pufillum.

Aрате. (Splintkiffer.)

inuricatus An. mer.

cornutus Malagafc.

hamatus Germ.

Monachus Anr. Inf.

Iefuita $N$. Holl.

fernoralis $A m$.

gonagra S. $N$.

capucinus Eur.

Dermeftes $L$.

2 maculatus Gall.

finuatus Gall.

tridens Am.

difpar. Gern.

fignatus Germ.

limbatus Germ.

Dermeftes domefticus

$L$.

volvulus Gall.

16. minutus $N . Z e l$.

Bostrichys. (Borkenkáfer.)

flavicornis $A m$. bor.

cylindrus Germ.

typographus Germ. ')

Der.

r) Ips typographe. Der felmparze Wurn. Der Holz. wourn. Der firgende Wurm. 
Dermeftes $L$.

BOSTRICHVS Laricis

Grum.

chalcographus Germ.

B) te aceus:

polygraphus Eur. s)

Dermeftes $L$.

monographus Gernu:

micrographus Eur.

Dermefles L.

Scolytus Angl. ')

crenatus Germ.

oleiperda Gall:

Oleae Gall.

pygmaeus Germ.

ligniperda Germ.

abietinus Germ:

villofus Germ.

piniperda Eur. ")

Dermeftes $L$.

teftaceus Germi.

volvulus $A$.

pubefcens Germ:

ater Supe.

melanoceph. Daxt:

vittatus Gejw. .

minutus Gerviv.

25. bidens Gernn.

B RंvCHVs. (Muffelkä:

$$
f e r \text {.) }
$$

fpinofus Iam.

Nucleorum Ind.

s) DerStadtchreiher P̈.

t) Das Kollienkäfercheri.

Le Seolyte:

11) Der Ficbtenzerflöbrer:
Brvchús Bactris.

umbellatarum Barb.

Pifi Am. Sept:

Cacao.

punctatus $S . \hat{N}$.

Robiniae Am. bor.

gibbofus Am. bor.

femoratus.

aeneus S. $N$.

Theobromae. marginellus Germ. analis $\mathrm{Ind}$. Or.

2 punctatus Helv.

4 maculatus Amer.

fcutellaris.

craflicornis S. $N$.

granarius.

Cilli.

futuralis Gall.

abdominalis Ind.

Or.

feminarius Angl.

villofus Germ.

rufipes Gall.

maculatus Am.

Munofae Am. mer.

flavicornis Germ.

ferraticornis Orient.

30. pectinicorniis $\left.{ }^{v}\right)$

$\Lambda \mathrm{N}$ THRIBVS. (Bürften-

käfer. L'Antluribe.)

Albinus Germus

ANTHRI*

†) L'Antribe noir ftrié. Porro:

1) Br. lutcicornis.P.

2) - imbricatus, P. 
A тит в в

Ges'm. ")

albiroftris (Germ. ${ }^{x}$ )

grifeus $N$. Holl.

Roboris S. $N$.

planirofris Germ.

fcabrofus Germ. y)

undatus Afr.

varius Germ. ${ }^{\text {) }}$ )

a. fepicola Germ.

BRACHYCER Vs,

apterus Ind.

Curculio L.

ocellatus Madag.

fcalaris C. B.S. .

obefis C. B.S.

globofus Ind.

roftratus Afr.

barbarus Aeth.

Curculio L.

inaequalis $C . B . S$.

crilpatus Barb.

cornutus Ind.

Cusculio L.

algirus.

retufus $C . B . S$.

fpectrum C. B.S.

'muricatus Hung.

15. Vva C. B. S.

w) L'Antribe noir frie.

x) Der voeifsriujshsbes Bkir. fenkäfer.

y) L'Antribe marbré.

2) L'Antribe minime.

Porro:- Anthribus ruf. collie. P. kiäfer)

Coryli Eur.

B) Avellan. I.

fpeciolus Tranqb.

2 puftulatus $A m$. Sept.

penfylvanicus.

gemmatus C. B. S.

notatus Ind.

longimanus Seneg.

nigripennis C. B.S.

fufcicornis Seneg.

fpinofus $C . B . S$.

indicus.

curculionoid. Eur:

angulacus Cajen.

pubefiens Germ.

Bachus Germ. a)

Betuleti Germ. b)

B) caeruleus,

Populi Germ. ')

bicolor Am.

hungaricus. pequatus Germ. ")

Curculio L.

coeruleacephalus Germ. coeruleus $\mathrm{S}, N$. triftis A. Girm. .

ATTE-

2) Le Becmare dorè à êtuis rouges. Charanfon crannifi.

b) Becmare vert, CharanFon da Boulaeu.

c) Becmare doré. Charanfon du Tremble.

d) Becuare dore a êtuis rouger.

E 
Attelabvs. fellatus $\Lambda$. Germ. cupreus Eur. Curculio $L$. aenets. fulciroftris Germ. cupriroftris Germ. rufirontris Angl. Alliarrae Eur.

Circulio $L$.

Pomonae S. $N$. Craccae Germ. ") Sorbi Germ. ") cyaneus Girm. ")

Curculio L. globofus Eur. Malvae Angl. Havipes Germ. "h)

Cutrculio L. purpureus Eur. Curculio $L$. frumentarius.

Curculio I. vernalis Germ. 4I. Betulae Germ. ') RHIN OMACER. (Dickkäfer.) :

e) Der Wickenafterriijfelk. Charanfon de la Veffe.

f) Der Vogelleer - After-: riijfelkäfer. let.

g) Charanfon noir vio. jaunes.

h) Becmare noir à pattes

1) Charanfon-à tête écorchée noir. Porro: Attelabus intermedius. $P$.
Rhinomacer. curculioides Ital. attelaboides Suec.

Cvrcvlio. (Riilfelkä-

$$
\text { fer.) }
$$

* Longirnflres femoribus fimplicitus. gigas Jipon. Palmarum L. Ind. cruentatus Carol. longipes C.B.S. Coloffus Ind, Or. Indus. paganus Ind. ferrugiueus Braf. hemipterus I.. Cajen. cribrarius S. $N$. variegatus $C . B . S$. melanocardius L. Ind. limbatus Seneg. cruciatus $N$. Holl. fanguindlent. Tabago. fafciatus. ftriatus $\ln \int \mathrm{Terr}$. Nov. rubetra Cajen. gagates Cajen.' mendicus Madagafe.

l'ineti Succ. Pini L. Suec. Onopordi $A f r$. Vrfus Ital. Iaceae Eur. ${ }^{k}$ ) planus Germ. punctulatus Amer.

Curcu.

k) Le Charanfon tachetédss têtes de chordon. 
Cvreveio. ocularis Barb.

Cyuarae Afr. Culon L. Germ. 2 maculatu Germ. punctun Germ.

2 guttatus $A m$.

2 lineatus Grrm. furdidus Am. mer. fuillus Am. mer. teffellatus Germ. nitens Gall. mer. abbreviatus Germ. Equifeti Angl. Cur. fraber $L$. dimidiatus. Pufo Sibir. atriroltris Germ. brunniroftris Dan. feftivus Surin. Tragiae Braf. curviroftris N. Holl. Aethiops Suec. Scirpi Gall. Pruni L. Eur. Arneniacae Germ. Camelus Germ. 4 tuberculat. Germ. teter Ital.

Campanulae Eur. nigriroftris Angl. variabilis Germ. piciroftris $H$ nfn. Salicariae Angl. floralis Suec. Pfeudacori Gall. Callor Gorm.
CVRCVLIO. pericarpius $L$.

quercicola Suec. aflimilis Suer. Sifymbrii Germ. Capreae Angl. 2 punctatus L. Suec. Carpini Grm. Eryfimi Germ. 4 maculatus L. Eur. unifafciatus Germ. Lythri Viland. 2 falciatus Germ. acridulus L. Eur. fcaluratus Gern. alauda Germ. dorfalis L. Eur. Quercu. Germ. futuralis Germ. Crux Germ. Lemnae Germ. exclamation. N. Hol. venuflus Angl. Plantaginis Germ. Rumicis L. Eur. Poilux (nec ad $\int_{\text {perfus }}$

Germ. granarius Eur.') Oryzae Orient. 2 tuberculat. $N$. Ze. land.

E 2

CvR.

1) Der fchmarze Kornmurin. Der Kornevester. 1.e Charanfon brin du bled; L.a Licorne; Calondre; Loffan; Chatepelea. fe Loffan. 
Cvrcviro. paraplecticus

L. Eur. ${ }^{\mathrm{m}}$ )

anguinus L. Germ.

Ariatellus Barb.

umbellatarum Barb.

mucronatus $B a r b$.

ferrugatus Hung.

Gages Guin.

8 linestus C. R. S. :

femipunctatus N. Holl.

4 puifulatus C. B.S.

mixtus Barb.

filiformis Ital.

cylindricus Sibir.

notatus Barb.

barbiroltris Ind.

anguftatus Angl.

Bardanae Germ.

truncatulus S. $N$.

volvulus S. $N$.

Afcanii L. Eur. auflr.

linearis Gall.

B) ferrugineus.

craflipes Gall.

Atriplicis L. Germ.

Lymexylon Germ.

* longiroftres fenorib. dent.

calcaratus.

bidens $N . Z_{\ell}$.

Taurus Cajen.

cornutus Cajen.

miliaris Cajen. .

cyanicollis.

jamaicenfis. drie.
CVRCVLIo. fenegalenfis, validus Cajeas. coronatus. mucoreus $\mathrm{Ind}$. Or. pufio $I n d . O r$. fpinipes $A m$. m. Bumbina Cajen. Scorpio Cajen. Chimaridis Amt $m$. guttatus Cajen. fafcicularis Cajen. Hyfrix Cajen marmoratus Cajen. fcaber Cajez. 3 fafciatus Cajen. cylindriroftris $N$. Holl. figma Ind. hebes treng. anuulatus Ind. caliginofus Angl. dubius.

roreus Cajen. brunneus $C . B$. $S$. Abietis Eur. dentipes Seneg. multiguttatus. pupillator Cajen. reticulatus Tranqb. Lapathi Eur. $L$. irroratus Cajen. 3 cinctus Griadel. Statua. 6 guttatus Anzer. luridus $N$. Holl. ftolidus $C . R$. S. frigidus Amboina. 
CvRcvz10. gibbus Inf. Bourbon. ocellatus Cajen: meditabundus N. Holl. ftupidus $N$. Holl. Mangiforae $\operatorname{lnd}$. Or. nultus Curom. Araneus Am. w. Strix Cajen. fqualidus Surin. germanus $L$. Scrophulariae L. Eur. Thapfus Germ. Verbafci Germ. Blattariae Ttal. Solani Germ. gravis C. B. $S$. 5 punctatus L. Eur. guttula Germ. abbreviatulus Germ. Echii Germ. didymus Germ. Lamii Germ. Brallicae Germ. Borraginis Gall. haemorrhoid. N. Holl. 3 maculatus Alf. litura Eur. villofus Germs. Raphani Germ. varians Sulec. Troglodytes Germ. carbonarius $L$. Eur. aterrimns $L$. Eur. Cerafi L. Eur. violaceus Eur. bor. Nucum L. Eur.
Cvrcvlio. probofcideus Am.bor. gulofus Gall. Parafita Cajen. efuriens Gall. nafutus. hauftellatus $C, \quad B$. $S$. varius Cajen. Ceraforum Angl. amoenus $N$. Holl. metallinus Am. $n$. bicornis $N$. Zel. tenuirolti is Angl. druparum L. Eur. Fraxini Suec. nelanoceplual. Germ. depreflus $L$. $A m, m$. Pomorum $L$. vorax Ital. tortrix L. Eivr. ${ }^{n}$ )

Tremulae Sicc. aeltuans $A m$. m. taeniatus Angl. elongatus $T a m$. ** longiroftres femor. pofticis falcatoriis. Alui Angl. Germ. pilofus Ang!. hortorum Germ. Calcar Germ. Saliceti Suec. Beccabungae L. Sibec. E 3 Cvr.

n) Dir Blattrolles. Le Charanfun fauve. 
Cvrcvlio. Salic is L.Eur. ${ }^{\circ}$ ) viminalis Eur.

Ilicis $V p l$.

Jota $V \mathrm{pl}$.

Fagi l. Eur.

Fragariac Germ.

Populi Seland.

**** breviroftres fenor. suutic.

argyreus $L$, Ind. fplendidus Braf. imperialis $\operatorname{Bra} \int^{\circ}{ }^{\circ}$ ) fumtuofus Cajen. Chrylis Ind. Or: regalis $L$. Ind. marginatus Cajen. I9 punctatus Cnjen. I 6 punctatus Braf. decorus Braf: nitidulus Cajen. candidus Cajen. niveus Cajen. lacteus Jamaic. pulverulentus Tranqb. fquamofus Chin. cretacens Gu del. cinerafcens Cajin. finaragdulus Cajen. 8 tuberculatus Cajen. feverus Cajen. modeftos $N$. Zel. fiavefcens $A m$ m. pollinofus Germ. Jateralis $I n d$. $O r$,

o) Le Charanfon Sauteur dn Son!e.

oo, Der Dainantk.
CvRcvilo, viridis $L$. Eur. gibher Cajen. fulvus Gorm. palliatus Girm. aurifer $A m$. m. $I n f$. cyanipes Cajern. repandus Ital. Tamarifci Afr. fplendidulus Sibir. curvipes Ind. Or. morbillofus Gall. nebulolus Eur. roridus Germ. marmoratus Germ. fulciroftris L. Eur. porculus Hung. emarginatus Germ. perlatus Chin. diftinctus Hung. glaucus Gern. obliquus Germ. albidus Germ. difiar Ind. Or. Vetula Trangb. Lufitanicus. crenulatus N. Holl. punctulatus Terr. Nov. incanus Eur. P) articulatus Germ. colatus Gall. melanchiolic. Germ. interruptus $N$. Holl. feutellaris Terr. Diem.

C'vr-

P) Le Charanfon gris, ftrié et fans ailes. 
Crkeveio. longimanus Crreyrio. ruficornis $I$.

Braf.

fpinifex.

micans Daw.

murinus Germ.

Polygoni Eur.

grifeus Angl. Itale.

3 gutratus Angl:

Arund: nis Germ.

moerens Germ.

grefforius Ital.

getninatus Germ.

gramineus Germ.

Diaderna Cajen.

Mele: Germ.

Coryli L. Angl.

lisceatus Eur.

lunatus Angl.

caninus Germ.

cinerafcens Ital.

fulvipes Germ.

ruficollis Germ.

fulvicornis Eur.

pilofus Germ.

undatus Ear.

hifpidnlus Germ.

hirfutulus Germ.

feabriculus Susc.

limbatus. Eur.

parvulus Ital.

viridicollis Germ.

cervinus L. Eur.

Ariatus Barb.

lacerta Ind. Or.

velox Cajert.

feminulum Hung.

\section{Eur.}

cloropus L. Eur.

canaliculatus $A m$. m.

multipunctat. Germ.

trillis Stuec. Angl.

raucus Germ.

adfperfus $N$. Holl.

punctatus Salec.

obfcurus Suec.

maxillofus Hung.

niger Germ.

laevigatus Germ.

rotundatus Germs.

variolofus Germ.

Aticlicus C. B. S.

valgus $A$ in.

B) viridi fquamolus.

fuccinclus C. B. S.

Camelear Iamaic.

vittatus L. Am. Inf.

Spengleri L. Amr. Inf.

$B)$ cinereus.

2 vittatus Amer. .

lividus $A m$. 'ns.

pulcher Iomaic.

feftivus Am. m.

hiftrionicus C. R. S. .

Rohrii Am. Inf. impreffus lannaic. albipes Madagafc. rivulofus $I n d$. Or. depreffiroftris Germ. inarginellus $A f r$ aeq. verrucofus $L$. C. B.S.

(Brachycerus verr. id.) 
CVRculio. capeufis L. 6 vittatus. vitulus Terr. d. Fuego. inaequalis $C . B . S$. acuminatus $N . Z$ in . capiftratus $C . B . S$. emeritus $L$. Ind. tuberculatus $I n d .{ }^{\prime}$ Or. crifpàtus C.B.S. cultratus $N$. Holl. tribulus $N$. Holl. 4 dens $N$. Holl. 4 fpinolus $C . R, S$. clavus $N$. Holl. nodulofus $C . B . S$. rubifer $C . B . S$. globifer $C . B . S$. pillularius $C$. $B . S$. glandifer C. B.S. * w * * * breviroftres femor. dentat. fpeciabilis N. Holl. pinguis Cijen. 3 dens $N . Z$ Zl. fulcomaculat. Germs. Ligultici L, Eur. Zebra Germ. 2 notatus Germ. nubilus Germ. nigrita Ital. fulcatus Garm. calcaratus Grrm. gemmatus Eur. picipes Germ. Morio Germ. 2 fulcatus litul.
Cvrcvero. collaris Germ. Iris Germ.

B) minor. Pyri L. Eur. lepidopter. A. Germ. Alneti Germ. vefpertinus $G$ :rm. Mali Germ. pallidus Germ. arboreti Cajen. dentifer Ind. Or. curvicoruis $I n d . O r$. dorfatus S. $N$. argentatus $L$. Eur. viridanus Tranqb. oblongus Eur. q) muricatus Germ. Ovatus Eur. albolineatus Germ. Picus Germ. 408. attelaboides Braf. BRENTVS.

* femor. fimplicibus.

\section{BREN.}

q) Le Chanrafon à ètuis fau. ver. Porro:

1) Curculio planiroltris. $P$.

2) - Fritillum. P.

3) - artemifiae. $P$.

4) - $\Delta$ bfinthis. F.

5) - Chloris. P.

6) - arcuatus.P.

7) - parallefus. P.

8) - bieclor. $P_{\text {. }}$

9) - brunneus, $P$.

10) - Linariac. P. 
Brentvs. barbicornis $N$. Mycetophagus, 16 , bi-

$Z e l$.

allumilis $N . Z_{e l}$. monilis $N$. Holl. brunneus Senpg. * femor. dentat. anchorago L. Ind. Or. Volvulus Greadil. nafutus Tamaic. difpar L. Ann. cylindricornis $N . Z \%$. 10. bifrons Cajess.

COLYDIVM. (Drahtkäfer.)

fulcatum Germ. elongatum Eur. filiforme Germ. frumentarium $A m$. ? Dermeftes $L$. Mrcetophagvs. (Pfifferkïfer.)

fafciatus S. $N$. 4 maculatus Germ. bicolor Am, $m$. dermelloides Germ. atcmarius Germ. multipunct. Suec. picicornis $A m$. $m$. fang:sinicorn. Germ. fulvicollis Germ. piceus Germ. punctatus Germ. nigricornis Grrm. caftanens Grrm. metallicus Grrm. teflaceus Gernt.

r) Colydium facriatum. P. fafciatus Gernm. ${ }^{\text {s) }}$

Hypophlaevs. (Pochkï$f(r$.
caftaneus Germ. linearis Germ. fafciatus Germ. depreflus Gerin. bicolor Gall. bicornis Gall. I.yctvs. (Kinlkäfer.)

* antenuarum articulo umi. co craffiori. politus Eur. Tenebrio $L$. depreflus Germ. nitidulus $5 . N$. 2 pnftulatus Germ. dentatus $A m$. 1 . brunneus Amer. Inf. tetebrans Germ. Iuglandis Germ. hifteroides Dan. * antennarum articuls 2. crafforibus. crenatus Eur. navalis $N . Z \varepsilon l$. canaliculatus: nitidus Gern: 4. contractus Gall. ") E 5 TRI. nipes:

s) Amplius: Mycet. Cpini.

1) Porro:

I) Lyct. abbreviatus. P.

2) - dermeftoides. P. .

5) - pubefecns. P. 
TR I о м. (Staubkä-

$$
\text { fer.) }
$$

2 pullulata Angl. glabra Suec.

marginata $A m$. m. rufipes Norwo. dubia Germ. vittata Ind.

Morio Gram. ferice Germ.

9. minuta Eur.")

$$
\text { Chryjomiela } L \text {. }
$$

Tetratoma. (Hackenk.) fungorum Grsm. gazella S. $N$. ancora Germu. v)

Scaphidivm. (Pilzkïfer.)

- 4 maculatum Germ.

4 puflulatum $N$. Holl. agaricinum Eur." )

Silpha $L$.

IPs. (Rindennager.)

faf́ciata Anu.bor. grandis Afr. aequin.

2 punchata Eur.

2 puftulata Germ. dorfalis Eur. 4 puftulata Eur. bor. Silpha $L$.

4 notata S. $N$.

6 pufulata Eur.

u) Porro:

1) Tritoma pilofa. P.

2) - flavipes. P.

v) Species nova: Tetrato. ma cinnamomea, $P$.

w) Scaph. Bolsti P. Por. ro: Scaphidiun fcutellatum $\mathbf{R}$.
Ips. 4 guttata Eur. ferruginea Germ. nigripennis Germ. Siipha $L$. haemorrhoid, Suec. rufipes Norw. 2enea Germ. humerulis Susc. teftacea S. $N$. lunata Germ. 21. rufifrons Angl. ${ }^{\times}$) VPIS.

cerambnides Suec. Attelabus $L$.

DiA PER IS. (Herzkäfer.)

Boleti Eur. Chryfomela $L$. violacea $G$ trm. MEL O E. (Mayrourmkä:fer.)

Profcarabáeus $L_{0}{ }^{y}$ )

majalis Eur.

marginata Ital. - punctata Angl. $\left.{ }^{2}\right)$ STAPнYLIN V S. (Raubkäfer.) aureus Siam. hirtus $L$. Eur. ")

STA-

x) Ifs marginalis. $P$.

y) Der gemeine Maywurm. Le Profcarabé. Le Scarabé on. ctueux. The Oyl Bectle.

z) Novae Species:

1) M. recta. P.

2) - brevicollis. P.

a) Le Staphylin bourdon. 
STAPHYLINVS. nebulofus STAPHYLINV f. fulvipes

\section{Germ.}

murin'ss $L$.

pubefeens Sure.

olens Eur. b)

fimilis Germ.

picipennis Germ.

maxillofus L. Eur.

oculatus N. Holl. Zol.

cyaneus $I$ tol ${ }^{\mathrm{c}}$ )

dilatatus Germ.

fulgeins Cajen.

erythropterus $L .{ }^{\text {') }}$

aeneocephal. Germ.

fottor Germ.

erythroceplialus $N$.

Holl. .

pilofus Cajen.

Iplendens Germ.

politus L. Eur. ')

triftis $D a n$.

varians Seland.

brunnipes Angl.

nitidus Ger m.

fulgidus Germ.

crenatus Germ.

frintulus Germ.

frumofus Germ.

cyanipennis Gall.

marginatus Angl.

\section{liffe.}

b) Le grand Staphylin noir

c) Le Staphylin blen.

d) L.e Staphylin à étuis con. leur de ronille.

c) Dtr glatts fik.

\section{Germ.}

flavefcens Eur. alpinus Lapp.

2 puflulatus Eur.

analis Germ.

2 guttatus ad litt. m. Balt. ${ }^{f}$ )

clavicornis Germ..8)

fulcipes L. Eitr.

flavipes Seland:

atricapillus Angl.

anguftatus Suec.

caualicnlatus Germ.

tenu is Germ.

gracilis Suec.

punctulatus Suec.

eınarginatus Gall.

bicolor Gall.

rufipes Grem.

tuberculatus S. $N$.

melanoceph. Sitec.

craflicornis Dan.

fanguineus Gall.

floralis Gall.

caraboides L. Eur.

rugofus Angl.

piceus Eur.

57. porcatus Suec. ". )

$\mathrm{OxY}$ -

f) Le petit Staphylin noir.

g) Die Zuno. Le Staphy. lin Iunon.

h) Species novac:

1) Staphylinus rivularis. P.

2) - pallidipennis.?. 
O XYYOR V s.: (Stumpfkï-

\author{
fer.) \\ rufus Eur. ') \\ Staphylinus $\boldsymbol{L}$. \\ maxillofu: Germ. \\ Iunulatus Eur. \\ fubterraneus Suec. \\ Staphylinzs $\boldsymbol{L}$. \\ cellaris Suec.
}

marginatus Suec.

3 maculatus Dais.

2 maculatus $S e l$.

2 puftulatus Germ.

analis Germ.

nerdarius Angl. thoracicus Germ. pyginaeus Germs. Havipes Erw. cryfomelinus Eur. ${ }^{k}$ )

Staphylinus $L$. melanocepl. Dan. abdominalis Norw. tefrecus Germ.
B) obtufus $\mathrm{M}$.

dimidiatus S. $N$.

Y POR V. brunneus

Germ.

minutus Seland. rufipes Eur.

Staplinlinus $L$.

Hypnorum Angl.

24. marginellus Germ. ')

PAENERVS. (Traubenkä*

$$
\text { far.) }
$$

riparius Eur. ${ }^{\mathrm{m}}$ )

Staphylinus $\boldsymbol{L}$.

ruficollis ad litt. $\%$.

$$
\text { Balt. ") }
$$

elongatus Eur.

Staphylinus $L_{\text {. }}$

fulvipennis Germ.

brunnipes Germ.

fulgidus Germ.

triccior Dan.

filiformis Germ.

orbiculatus Stue.

Io. melanoceph. Gorm. $\left.{ }^{\circ}\right)$

1) Porro:

1) Oxyporus futuralis, $P$.

2) - - erythropte.

rus. $P$.

i) Le Staphylin jaune a $\quad$ iii) Staphylin de rivages. rete, étuis et anus noir.

k) Le Staphylin conleur corcelet rouge. de paille. ii) Le Staphylin noir a

o) Paederus dimidiatus, $P$. 


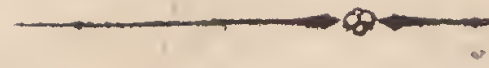

\section{CLASSIS II. \\ $\begin{array}{lllllll}V & L & O & N & A & T & A .\end{array}$}

palpi quatuor, maxilla tecta, galea obtufa.

* Sintennis filiformibus.

121. Acrydivm. Palpi aequales, filiformes. Labium ovatum, apice fiffum.

125. GR YLLvs. Palpi aequales, filiformes. Labiun rotundatum, bifidum: laciniis rotondatis.

** Anternis enffformibus.

122. Trvxalis. Palpi filiformes. Galea truncata, fornicata. Labium bifidum.

*** * Antemnisfetaceis.

118. Forficvla. Palpi inaequales, filiformes. Labiun trifidum.

19. BLAtтA. Palpi inaequales, filiformes. Labium bifidum: laciniis fifis; pinnis inaequalibus.

120. Mant1s. Palpi fubaequales, filiformes. Labium bifidum: laciniis fiffis; piunis aequalibus.

123. Acrieta. Palpi inaequales, filiformes. Labium quadrifidum.

124. Locvsta. Palpi inaequales, filiformes. Maxilla apice tridentata. Labium bifidum: laciniis feta fu. bulata.

II. VLO. 


\section{V L O NATA.}

Forficvla. (Zongenkäfer. BLATтA. americana

Le Perce oreille.)

Herculanea S. $N$.

auricularia L. Eur. a)

gigantea Eur.

2 punctata Ital.

2 guttata Hung.

flavipes Guin.

albipes Ann. m.

minor L. Eur. b)

pygmaea Guin.

flexuofa Cajen.

dentata Madera.

annulats $A m$. m.

erytroceph. Am. m.

clongata $A m$. Inf.

parallela Madera.

Morio Otaheite.

pallipes Prom vir.

18. Havipennis Sneg.

BLAtTA. (Sthalie. la Blat-

\section{te. Ie (acrelat)}

gigantea L. Am. Afia.

maderae.

aegyptiaca.

occidentalis Am.

furinamenfis $L$.

a) Le Grand Perce-oreille.

b) Petit Perce-oreille.
$L$.

aufralafiae.

erythroceph. C.B.S.

capenfis.

indica.

gallica.

nivea $L$. Am.

irrorata N. Holl.

viridis Amo.

brafilienfis.

Petiveriana Ind.Or.

Caffida $L$.

orientalis $L$.

cincla Am.

picta Braf.

variegata $N$. Holl.

longipalpa S. $N$.

lapponica $L$.

gertnanica.

reticulata S. $N$.

livida Gall.

ruficollis Ind.

diaphana Am. m.

maculata Germ.

marginata Ital.

oblongata L. Surin.

nitidula Strin.

32. hemiptera Germ. 
Mantis. (Fangheufchrek: MANTis. B) religiofa

ke. Das wandeinde Blatt. La Mante.)

filiformis $A n$. m.

ferula Guadel.

calamus Inf. St. Cruc.

Rolfia Ital.

angulata Guadel.

gigas L. Afiae.

necydaloides Afiae.

fpinocula S. $N$.

atrophica Iava.

f́inofa Ind.

2 Ppinula $A m$.

jamaicenfis.

lateralis Braf.

aurita Ind Or.

linearis Ind.

rofea Ind. Or.

flabellicornis Trangl.

gongylodes L. Afr.

Af:

pauperata Corom.

mendica Alex.

truncata Cajerz.

ftrumaria $L . I n d$.

tricolor L. Ind.

cancellata Ind.

ficcifolia $L$. Ind.

pectinicornis L. Jamaic.

oculata L. Afr. aeq.

fuperftitiofa $A f r$ aeq.

undata Tranqb.

irrorata L. Amer.

Ariata Ital.

oratoria I. Or. Eur. anft.
L. ')

pracearia $L$. Am.

fancta Gall.

fimulacrum Amo. monacha $C . B . S$. obfcura Afr. aeq.

hyalina Amer.

feneftrata $A f r$ a aiq.

bidens Awer. grifea.

miniftralis $N$. Holl.

urbana Ind.

ruftica ad litt. Patag. '

nafuta $C . B . S$.

lobsta C. B. S.

pulcra Tranqb.

faufta C. B.S.

perfpicua Cajen.

pagana Gall.Germ.

Raphidia mantifpa $L$.

minuta $A$ m. m.

52. pufilla C. B. S.

ACRYDIVM.

morbillofum Sierra Leon.

2 punctatum Eur. ${ }^{\text {d) }}$ )

Gryllus $L$.

fubulatum Eur.

Gryllies $L$.

Trvxalis.

nafutus.

ß) Gryll. turritus L. conicus N. Holl.

\section{TRVXA.}

c) Pregadiou.

d) Criquet à Capuchon. 
TRvXALIs. vittatus

Chm.

hungaricus. brevicornis $A m$.

Grylines $L$. crenulatus Tranqb.

ACHAETA.

Gryllotalpa Eur. Am. ') Gryllus $L$. monitrofa Ird. domeltica Eur. ') Gryllus $L$. affunilis Iamaic. reticulata. brafilienfis. orientalis Tranqb. Havipes St. Thom. capenfis. Morio Afr. aeq. . campeftris Eur. ${ }^{\mathrm{g}}$ ) Gryllus $L$. umbraculata Barb. Grylhus $L$. hofpes Aner. maculata S. $N$. Crucis Ind. guadelupenf. italica. minuta Amer. . Gryllus $L$.

9. fylveftris Gall. lere. Ion.

c) Die Werle. Courtil-

f) Das Heimeben. Le Grilchaups.
L. OC V S T A. (Sclmeehew* fchrekke.) citrifolia Ind. Gryllus L. lauritulia Amer.m. Gryllus L. myrtifolia Amer. Gryllus $L$. feneftrata Ind. Or. camellifolia Am. oleifolia Tranqb. thymifolia $N$. Holl. graminifolia C. B. S. Jilifolia Ital. perfpicillata Amrr. ferripes $\operatorname{In} \int$. $A m f \hat{t}$. fpinipes Braf. maxillofa Amer. elongata Ind. Gryllus $L$. femorata Tranqb. Gryllus rugojus $L$. italica. Ipecularis An. perforata $C . B . S$. ocellata Ind.

Gryllus I. lanceolata Sierra Leon. pallens Cajen. acuminata Ind. Eur. Gryllus L. conocephala Afro Giyllus L. triops Ind. Gryllus $L$. coronata lad. 
Gryllus $L$.

Lo C V T A. melanoptera lind.

Gryllus L. coriacea lnd.

Gryilus L. reticulata Gurdel. allifrons Madera. . falx Madera. grifea Ital. viridiltima Eur. ")

Gryllius $L$. verrucivora Eur.

Gryllus $L$.

gladiator Tranqb.

varia Germ.

brachyptera Eur.

Gryllus $L$.

ferrata Hung.

fufca Gnll.

pupa Aethiop.

Gr. pupus apterus $L$.

fpiuulofa Ind.

Gryllas $L$.

Onos Sib.

ephippiger Etur. auft.

aptera Ital.

pedeftris Ital.

ferricauda.

46. Laxmanni Sibir. ${ }^{\text {i) }}$

GryLLVs. (Grasluipfer. Le

Grillon.Grashopper.)

h) Sauterelle à coutclas.

i) Auplius:

I) Locufta clypcara. $P_{\text {: }}$

2) - denticulata $P$.
GRYLLVs. Elephas $L$. Afr.

fuccinctus $L$. Ind. criftatus L. Arab. dux Am. $m$. carinatus Orient. Lupus $L$. Am. m. reticulatus Tranqh. gallinaceus Ind. Or. ferripes $I n d$. ferratus L. C. B.S. turcieus Ind. inanis $C$. B. $S$. papillofus C. B.S. variolofus $L . C . B . S$. miliaris L. Am. morbillofus L.C.B.S. cinctus Sengg. leprofus. punctatus Ind. Or. fcabiofus Tranqh. fquarrofus $L$. Afr . liaematopus L. Ind. flavicornis Chin. flavefcens Tranqu. pictus Cajen. tartaricus L. Afr. migratorius L. Tawt. ") ruficonis sievr. Lenn. lineola Ital. variegatus $L$. Am. vocans $N$. Hull. luridus ifr. aeq. dorfalis S. $N$.

GRYL-

kj Santerelle de pafiuge. F

Grit. 
GRY LL V s.' nervofưs

\section{Guin.}

Banian S. $N$.

muficus $N$. Holl.

tuberculatus Iutland.

Cilti.

Aridulus L. Eum.

Morio Afr.

ferrugineus $A f r$. aeq.

furinamus $L$.

jtalicus $L$.

germanicus.

thalafinus Ital.

virginianus.

coerulans L. Ital.

carolinus $L$.

obfcurus L. Afr.

falciatus Gall.

fibiricus $L$.

coerulcfeens $I_{\text {. }}$ Eur. cinerafceus Ital.

fulphureus $A m$.

flavis $L$. Ann.

cyanipes $A m$.
GRY L L vs, ruflicus Ani.

Jateralis $A$ m. velox Chin.

2 guttatus L. Enrr. viridulus L. Eur. fanguincus $S$. $N$. gronlus L. Eur. ') captivus $N$. Holl. Tamulus S. $N$. clavicornis Surin. ciliatus S. $N$. faber S. $N$. rufus L. Eur. pedeftris L. Eur. 21. perfpicillatus $L$ : Ind. ${ }^{\mathrm{m}}$ ) té.

1) Le Criquet enfanglanm) Porro:

I) Gryll, pellicens, P.

P.

3) - lineatus, $P$.

4) - pedeftris. P. 


\section{CL $\Lambda$ S S I III. \\ $\begin{array}{lllllllll}S & \mathrm{Y} & \mathrm{N} & \mathrm{I} & \mathrm{S} & \mathrm{T} & \mathrm{A} & \mathrm{T} & \mathrm{A} .\end{array}$}

Palpi quatuor, maxilla connata cum labio.

128. Ephemera. Mandibulae vullae.

130. Phryganea. Maxillae tome comnatae cum labio:

129. Sembl1s. Maxilla bifida. Labium annullatum, integrum.

126. Lerisma. Palpi inaequales. Labium membranaceum, rotundatum, emarginatum.

127. Podvra. Palpi fulclavati. Labium bifidum."

13x. Hemerobivs. Maxilla recta apice fifia. Labium conneum, fornicatum.

132. Termes. Labium corneum, quadrifidum: laciniis linearibus, acutis.

I35. Raphidia. Maxilla cornea, integra. Labium breve, corneum.

235. Panorpa. Os porrectum, corneum. Maxilla bifida, truncata.

133. Myrmeleon. Palpi fex inaequales: poftici multo longiores; articulis ultimis duobus extrorfum craflioribus. 


\section{S Y N I S T A T A.}

L E pol s ir A. (Schuppen. Erhemera. (Haft, Tag* thierchen. La Forbi- fligeg. L'ephemere.) cine.)

* cauda trifeta.

facharina $\left.L . A m . E u r{ }^{2}\right)$

polypoda L. Euir.

- lineata Helv. villofa Chin.

5. collaris Am. mer.

PoD v A. (Fusfchwanz. thicrgen. La Podure.)

fignata.

\&viridis L. Eur. polypoda L. Eur. atra L. Eur. plumbea $L$. Eur. minuta L. Eur. nivalis $L$. Eur. vaga $L$. Eur. arborea L. Eur. villofa $L$. Eur. cincta L. Eur. annulata Entr. lignorum Eur. pufilla $L$. Eur. aquatica $L . E u r .{ }^{b}$ ) fimetaria $L . E u r$.

17. ambulans $L$. Enus.

a) La F. platte. Das Zibckertbiergen. quie.

b) La P. noire aquati. vulgata $L$. Eur. ? lutea $T_{\text {.. Eur.. }}$ maroccana. marginata L. Susec: vefpertina $L$. Eur. haltersta $G$ irm. brevicauda Gall. * * cauda bifeta. venofa Dain. bioculata $L$. Eur. nigra L. Eur. fiffeata $L$. Eur. albipennis Gall. horaria $L$. Eur. culiciformis $L$. Eur. ftriata $L$. E $w$. 16. diptera $L$. Suec. SEMrLis.

pectinicornis Am.S. Hemerobius $L$.' grifea $A f r$. atrata $A f r$. aeq. punctata. fufcata Ind. Or. phalaenoides Eurr. Phryganea L. marginata Germu.

Sembis.

c) L'E. commune, 
SEM BI. IS. bicaudata

$$
\text { Eur. ') }
$$

Phryganze L. nebulofa Eur.

Phryganea L.

lutaria Eur.

Hemerobius $L$. viridis Hafno.

Phryganea. (Wajermot.

te. La Frigane.) reticulata $L$. Eurr.

ftriata $L$. Eur.

analis Suet.

fufca $L$. Eur.

'difcoidea $V_{p} p$.

pilofa Sutec.

pallipes 1tal.

fignata Ams..

grandis $L$. Eur.

varia Dan.

irrorata $A m$. mer.

flavicornis Germ.

rhombica L. Ear.

grifea L. Eur.

atomaria Germ.

fennica.

vittata S. $N$.

atrata Gall.

notata Am. bor.

tigurinenfis $\mathrm{S} . N$.

2 maculata L. Eur:e

nigra $L$. Eur.

azurea $L$ Entr.

marginata $L$. Suec.

variegata Germ. jaunes.

d) La perle brune à rajes
Phruganea, 2 lineata $L$ :

Eur.

interrupta Angl.

hirta Angl.

longicornis L. Euts.

4 fafciata Angl.

Waeneri.

punctata Gall.

filofa L. Angl.

3 punctata Germ. minuta Eur.

36. pufilla Ital.

Hemerobivs. (Florfiege

$\therefore \quad L$ Hemerobe.)

cornutus Am. b.

Raphidia $L$.

nebulofus S. $N$ :

perla L. Eur: $\left.{ }^{\circ}\right)$

flofus Otaheite.

albus $L$. Eur.

capitatus Germ:

candidus S. $N$.

chryfops $L$. Eus:

maculatus Gall.

phalaenoides L. Euwr.

nitidulus Gervn.

hirtus L. Eur.

fufcatus Dan.

lutefcens Germ.

Humuli L. Vp .

longicornis Germ.

ftriatulus Eur.

8 punctatus Coch. Che

fafciatus Germi.

F 3

HEME. ,

e) Die Perlfiege. Le Lion des pucerons. 
HEMEROBIv.s. variegatus Gall. nervolus Gall. 6 punctatus L. Eutr. picicornis Dan. flavicans L. Eur.

is $\quad 2$ punctatus L. Eur.

4 punctatus Germ.

- abdominalis Angl. pulfatorius Eur. Am.

\section{Tormes $I$.}

29. fatidicus Eur.

Tormes $L$.

TERMES. (Holzlaur." LE. Bou des bois.) fatale 1nd. Afr. Am. deftuctor Am. mer.

Hemerobinis marginalis. L.

Morio Cajen.

Hemerobius taftaceiss $L_{\text {to }}$ Arda Afr.

mordax Afr.

6. flavicolle Barb.

MÝ MEL E ON: (Afterjungfer. Lo Fourmil. lon.)

libelluloides L.C.B.S. Pardalis Corom. pantherinum Geirm. Jynceum Sierr. Lion.
MYRMELEON. formicarium L. Eur.

Catta Madera. ermineum S. $N$. punctatnm Tond. Or. mnftelinum S. $N$. urfinum S. $N$. Formicalynx $L$. Afr. tigrinum $N$. Holl.

13. caninum Guin. -

Ascalaphys.

barbarus Eur. Afr. italicus.

Myrmeleon longiconne L.

capenfis. appendiculat. aufralis Eur.

6. cajennenfis.

Panorpa. (Scorpionfliege.

La.mouche Scorpion.) communis L. Eur. germanica. Scorpio Am. bor. falciata Am. bor. byemalis L. Germ. tipularia Pedem. 7. Coa L. Archip. RapHIDIA. (Kameelfliege.) Ophiopfis L. Eur. ${ }^{\mathrm{f}}$ ) f) La Raphidie commune. 


\section{$\infty$}

\section{CLASS IS IV. \\ P I E Z A $T$ A.}

Palpi quatwor, maxilla cornea, compreffa, faepe elongata.

137. Cynirs. Palpi inaequales, fubcapitati.

138. Tenthredo. lalpi filiformes. Labium trifidom.

I39. Sirex. Palpi poftici longiores extrorfum craftiores. 140. ICHNEVMON. Labium cylindricum, apice menlbranaceum, emarginatum, medio palpigerum.

341. Evania. Palpi poftici incurvi: articulo tertio quadrato. Labium conicum, apice membranaceum, integruin.

142. Chalc1s. Palpi aequales. Antennac breves cylindricae, fuffiformes: articulo primo fubcrafiori.

144. LARKA. Maxilla brevis, cornea, rotundata, fornicata. Labium exfertum, emarginatum.

145. Tiphia. Maxilla membranacea, rotundata. Labium breve, corneum, tridentatum, medio palpigerum.

147. Chrysis. Labium porrectum maxilla lineari multo longius, apice membranaceum, emarginatum.

149. Levcospis. Palpi aequales, breves. Labium maxilla longius, emarginatum. Antennae clavatae.

15 I. VESPA. Labium porrectum maxilla longius, emarginatum, feta utrinque inftructum.

153. Merlinvs, Palpi elongati: articulis cylindricis. Maxilla brevis, rotundata. Labinm ovatum longitudine maxillae, fub apice palpigerum.

154. Phinanthvs. Palpi filiformes: articulis cylindricis. Labium compreflum, rotundatum, integtum maxil. lis longius. 
155. CRAвиo. Palpi articulis compreflis rhomboidalibus. Lahium breve, corneum, apice palpigerum.

161. Formica. Palpi inaequales: articulis cylindricis. Labium fubmembranaceum, cylindricum, apice palpigerum.

163. Mvtilla. Palpi articulis obconicis. Labium porrectum, corneum, obconicum, apice palpigerum.

162. Do'r yuvs. Palpi inaequales, brevilfimi. Maxilla brevifima, cylindrica. Labium rotundatum, integrum.

* * lingrza inflexa.

143. SpHEx. Lingua inflexa, trifida. Labium emargina: tum utrinque feta infructum.

146. Scotra. Lingua inflexa, trifida. Labium porrectum, apice membranaceum, integrum, medio palpigerum.

148. 'Trynnvs. Lingua hroviflima, involuta. Labium trifidum: lacinia intermedia emarginata.

152. Masaris. Labium compreflim, exfertum, defi. nens in linguam bifidan, retractilem. Antennae extrorfum crafliores.

156. Hycaevs. Lingua inflexa trifida. Maxilla brevis, cornea. Labium elongatum, trifidum: lacinia intermedia emarginata.

157. ANDRENA. Lingua trifida. Labium cylindricum, maxilla longius utrinque fetis duahus membranaceis.

5.o. NomanA. Lingua inflexa, trifida. Palpi poftici linguaeformes: articulo fecundo longiflino.

58. Aprs. Lingua inflexa, quinynefida. Palpi brevifimi.

150. BEмвеx. Lingua inflexa, quinquefida. Labium porrećtuin, os involvens.

159. Evcera. Lingua feptemfida. Labium quinquefidum laciniis intermediis maioribus, apice palpigeris. 


\section{IV. $P \perp \mathrm{E} Z \mathrm{Z} \quad \mathrm{T} A$.}

Cynips. (Gaherfpo. Le Tenthreno. (Blatiefpe.

Cinips.)

adfcendens Germ.

compreffa A. Ital.

Rofae L. Entr.

Glechomae L. Eur.

Quercus baccarum $L$,

Eur.

Quercus folii $L .{ }^{\text {a) }}$

Quercus inferus $L_{\text {o }}$

Eur.

Quercus petioli $L$. Eur:

Quercus pedunculi $L$. Eitr.

Quercus ramuli $\boldsymbol{L}$. Eur.

Quercus tojae Galt. Fagi L. Eur. 'viminalis L. Eur.

Capreae L. Eur. Salicis ftrobili L. Eur. . Amerianae L. Eur. ruficornis Gall. negacephala Dan. italica.

Plenes L. Or.

Sycomori L. Aegypt. longipennis Gall. 23. aptera Gall.

a) Die Eichenhlattgw. Le C. de la Galle liffe et rorde du chene.
Lo Firelon. La Mouche à fice.

* Antenuis clavatis. femorata $L$. Eur. ')

lucorum L. Eur.

lutea L. Euv.

fylvarum Germ:

Amerin.. L L: Eus:

marginata $L$. Eur.

triftis $\ln \int$. Sundnocr:

Vitellinae L. Eur.

fafciata L. Eur.

fericea $L$. Eur.

B) nigricornis.

obfcura Suec.

** Antennis inarticulatis extrorfum orafioribus.

uftulata L. Eavr.

enodis L. Eur.

clavicornis Am. $\int$.

coerulefcens Angl.

americana $L$.

coltalis Germ.

Rofae L.Eur. ${ }^{\text {) }}$ )

Eglanteriae Germ. fpinarum.Germ.

F 5

TEN-

b) Le Frelon noir à echancrure. fier. 
Tenthredo. ferva * Tenthredo. Capreae $L$.

Seland.

annulata Germ.

** Antonnis pectinatis.

cephalotes Germ.

nemorum Gerss.

frutetorum Germ.

dorfata Angl.

Inniperi L. Eur.

Pini L. Fur.

**** Antennis filifor. mis articulis 7. 9 .

Scrophulariae L. Eur.

Salicis L. Eur.

flaviconis Germ.

luteicornis Germ.

viridis L. Eur.

cingulata Germ,

Rappae L. Eur.

Cerafi L. Eur.

naflata $L$. Eur.

ovata L. Eur.

Alni L. Eur.

pavida Suec.

Abietis L. Eust.

analis Germ.

germanica.

maura Germ.

rufiventris Germ.

livida L. Eur.

albicornis Ital.

gonagra Ferm.

atra L. Eur.

marginella Germ.

eincta $L$. Eur.

ruftica Eur.

laterąlis Suec.,
Etr.

Morio Germ.

feptentrional. 'L. Eur.

I 2 punctata $L$. Etur.

punctum L. Germ.

blanda Angl.

4 maculata Germ.

vaga $A n g l$.

opaca Suec.

carbonaria L. Germ.

nigra $L$. Suec.

acthiops Angl.

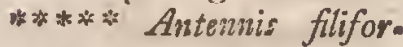
mibus, articulis plurimis.

erythroceph. L. Eur. d)

fylvatica $L$. Eur.

Cynosbati L. Eur.

figuata Germ.

Populi L. Eur.

vafra $L$. Eur.

Betulae L. Eur.

campentis L. Germ.

pratenfis Germ.

faltuum L. Eur.

haenorrhoid. Germ:

flava $L$. Eur.

78. arbuftorum Angl.

Sir Ex. (Schwanzwejpe.

L'V rocere.)

gigas La. Eur.

pfyllius Eur.

Columba L. Am.

SIREX.

d) La monche à f́cic à tete rouge. 
Sirex. magus Germ. nigricornis $A m$. $\int$. flavicornis Labrador: fufcicornis Germ. fpectrun $L$. Entr. juvencus L. Ettr.

a) nigricornis

B) flavicornis. fantoma Germ. cyaneus Am. $\%$ albicornis Am. $\mathrm{m}$. nigrita Germ. camelus .L. Eur. emarginatus Germ. dromedarius Germ. pacca.Germ. fimilis C. B. S. velpertilio Germ. marifcus L. Eztr. troglodyta Germ. noctilio Germ. compreffus Rarb. pygmaeus L. Eutr. macilentus $B a r b$. 26. tabidus Angl.

I С N E V MON. (Schhupfwespe. L' Ichnetmonz.)

* Scutello albido, antemis albo annulatis. fugillatorio, L. Eur. intratorius Rasb. molitorius $I$. Eur. pedatorius Germ. culpatorius L. Eur. raptorius L. Eur. farcitorius L. Eur. atratorius Germ.
ICHN E V MON. crifpatorius $L$

Eur.

extenforius $L$. Eur. ')

armatorius Germ.

motatorius Dan.

deAractorius Selmad.

fignatorius Germ. faturatorius L. Erur. glaucatorius Germ. grofforius Seland." quaefitorius $L:$ Eur. nutatorius $N$. Holl. olitorius Germ. albatorius Ind. porrectorius Gerzzs. irroratorius Am. oratorius Germ. infractorius L. Eur. ambulatorius $A n g l$. praecatorius Germ. feductorius Gall. obfolctorius $G$ crm. piforins L. Eur. bor. expectorius A. Ital. affertorius Germ. ferrugatorius Guin. *** Scitello albo antennis migris totis. luctatorius Eur. N. Zel. lotatorius $N$. Zel. negatorius Angl. laboratorins Germ. occiforius Germ.

ICHNEV-

e) L'Ichneumon panachée de noir et citron, à a pmeau btanc gux anternnes, 
ICHNE VM. of culatoriusGerm, IC H N V MON, nigrator nugatorius A. Ital. falciatorius Angl. volutatorius L. Eur: : mercatorius Ital. vaginatorius $L$. Eur. annulatorius Angl. maculatorius Germ. necatorius Germ. frontatorius sel. bidentorius Eur. marginatorius Germ. perfuaforius L. Eur. vagatorius Germ. defignatorius $L$. Eurr. edictorius $L$. Eurr. dubitorias $N$ : Foll. flavatorius A. Ital. laetatorius Germ. fuforius L. Eur. melioratorins Otaheit. lutoritus Paedem. deliratorius L. Eur. falcatorius Dano: fponforius Gernt. follicitorius $N$. Zet. nuptatorius Sal. erectorius S. $N$. fegmentorius Germ. forforius $L$. Eur. punctorius Germ. cinctorius Angl. decoratorius $N$. Zel. , * * Scutello thorace comscolore, antennis fajcia anmulatis.

Germ. reluctator $L$. Eur. objurgator $A f r$. aeq. comitator L. Eur. b. anuulator Gerns. zonator. Ital. clavator Gernn. vigilator Afr. aeq. reftaurator Dan. caudator N. Hollo debellator Suec. irrigator Germ. ". : , , migrtor Ear. Sputator Germ. dimidiator Gerim. abbreviator.Gern. fpoufor Gerizs. profligator Eur. incubitor L. Eur inftructor $B a r b$. exhortator Germ: ferrugator $A m$. abbreviatus A. Gern: *** Antennis nigris to tis, foutello. thorace? concolore. itinerator Guins. pennator $A m$. defertor L. Eur. 6 . nominator Gall. purgator Gall. faltidiator Afr. aeq. ariolator L. Am. wer. proficifcator $N$. Holl. hof pitator $N$. Holl. 
ICHNEVMON. - ormator ICENEVMON." atrator

Cajen.

pedator Ind.

denunciator $A f r$.

coftator (nec nominator)

Cajen.

antennator Cajen:

defenfor $N$. Holl.

aculeator Tranqueb.

femorator Tranqb.

arrogator Ital.

infidiator Afr. aeq.

capitator N. Holl.

mutator $N$. Holl.

lapidator Germ. denigrator L. Eur.

- flavator Barb.

initiator Eur.

corrufcator L. Eavr.

rutilator $L$. Eur.

manifeftator $L$. Eur. ${ }^{\text {f }}$ )

approximator Seland.

polycerator Ind.

Junator Am. bor.

compunitor $L$.

ovator Ital.

fufcator Inf. Sandw.

fegmentator Sulec.

enervator Germ.

infligator Germ.

irritator Am.

coerulator (nec lapidator)

Eur. anftr.

delufor $L$. Eur.

elongator Angl. quetre,

\section{Eur.}

mandator Germ.

reftaurator Germ.

molchator Dan.

caftigator Germt.

cuftodiator Germ:

nuntiator Germ.

fabricator Germ.

latrator Angl.

titillator L. Eur:

haflator Env.

cultellátor Grrms.

venator $L$. Eur.

fornicator Ital.

lineator Angl.

extenfor L. Eur.

maculator Germ.

turionellae $L$. -

anator Gernt.

Arobilellae $L$.

oculator Angl.

Cyusips inanita $L$ :

lucidator Seland.

roborator Bnrb.

fomentator L. Sulec.

menfurator Germ.

pennator Gernz.

refinellae $L$.

praerogator $L$.

barbator.

cylindrator Gerva:

accufator Germ.

ftercorator Germ.

incitator Germ.

variegator SueE. 
ICHNEVMON, acumiuator Germ. .

frigator Germ. fcabrator Germ. gravidator L. Emr. inculcator $L$. E Ew . falcator Suec. pugillator $L$. moderator $L$. faltator Angl. erigator Gorm. jocator Germ. compenfator Germ. regulator S. $N$. mercator Germ. flagellstor Germ. cunctator Germ. irrorator Eur. ausftr. rufpator L. Eur. curvator Dan. jaculator $L$. affectator L. Eur. necator.

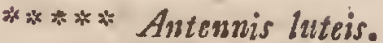
lateus L. Eur. ramidulus L. Germ. flavus Am. mer. ferrugin. A. Ital. tefaceus S. $N$. fulvus Amer. annulatus $17 n d .0 r$. atratus Am." bor. dentatus Norlv. varicornis Germ. Morio Am. $f$ : compreflus Suec.
ICH NEVMON!. varius

Germ. amictus Angl. glaucopterus L. Eur. relictus $\mathrm{S} . N$. circumflexus $L$ Eur. pundatus Corom. Hiftrio Selaidd. flavicans Germ. tricolor Ital. formicatus $L$. Suec. mutillarius Dan. affinis Gern. abdominalis Gall. cinctus L. Eur. $b$. fufcatus Gorm.

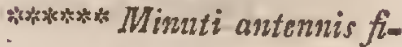
liformibus, abdomine ovato feflili.

femiauratus L. Eur. nitidulus Ital. fplendidus A. Ital. ignitus Barb. chryfis Barb. mufcarum $\boldsymbol{L}$. Bedeguaris $L$. nigricornis Dan. gallarum $L$. Iuniperi $L$. quadrum Germ. 2 maculatus Germ.

B) aeneus. puparum $L$. larvarum $L$. Cynipedis $L$. Spheguna Germ.

JCHNEV. 
Icheveron. Coccorum L. CH́AI Cis clavipes

\section{Eur.}

fecalis $L$.

B) agricolator $\mathbf{L}$.

fubcutaneus $L$.

figina.

conicus Dan.

globatas $L$.

glomeratus $L .{ }^{\text {b }}$ )

fericeus Guadel.

Aphidum L. Eur.

ovulorum ' $L$.

minutus Gall.

pedtinicornis $L$.

ramicornis.

hemipterus Gall.

agilis Suec.

curfitans Suec. pulicarius Germ.' falciatus Germ.

Acarorum Eur. 6. Mutilla L. pedicularius Germ. pedeftris Suec.

256. formicarius $L$. Suec.

Evania.

appendigafter C. B.S.

$$
\text { Sphex } L \text {. }
$$

maculata Angl.

fafciata Ital.

fefflis Gall.

minuta Gall.

6. punctum Ital.

Chatcrs. (Zahnuefpe.)

fifpes Eur.

Sphex $L$.

g) $T$. a coton jaune,
Germ.

apiformis.

minuta Dan. " )

$V$ espa $L$.

punctata Am. 2 .

podagrica Tranqb.

aenea Ital.

pufilla Germ.

annulata $A m$. mer.

flavipes Am. mer.

maculata Cajen.

12. nigricornis $\mathrm{S} . N$.

Sт нах. (Sandiespe Le

$$
\text { Sphex.) }
$$

* abdomine petiolato.

fabulofa L. Eur.

arenaria Germ.

lutaria Gerw.

Thomac Inf.

fervens $L$. Ind.

cyanipennis Cajers.

haemorrhoid. Afr. aeq.

argentata Corom.

rufipennis Tranqb.

flavipennis Ital.

penfylvanica.

violacea $C . B$. S.

cyanea $A m$. bor.

aurulenta Tranqb.

tomentofa Sies, Leon.

femorata Ital.

tibialis C. B.S.

atra A. Ital.

SPHEX.

h) L'a Guepe noire à cuif. fes pofterieures fort grofles. 
Sphex. Figulus $\boldsymbol{L}$. Ssuec. lowata $A m$. InS. affinis Am..Inf. jamaicenfis. erythroce ph. Malab. foirifex Lo Eur. anftr: B) aegyptia L. madirafpatana Malab. Clavus N. Holl. holofericea Barb. pubcicens Guin. cincla Guinz. lobata Afr. B) ininor. ciliata Ind. compreffa Malab. fibirica. ichneumonea L. Am: Apis $L$. $B)$ nigra.

hirtipes Guin. albifrons Guin. maxillofa Barb. unicolor Hifp. * * abdomine feffili. Morio N. Holl. collaris N. Holl. frontalis $N$. Holl. analis $1 n d$. $O r$. fimilis Afr. aeq. aflimilis Tranzb. dimidiata Barb. fufca $L$. Eur. viatica $L$. Eutr. Vrfus. amethyftina St. Crux.
S PHEX. "cingulata $N$. Holl. nigra Eur. labiata Am. mer. variegata Germ. fanguinolenta Germ mixta A. $A \dot{m}$. Inf. maura Tranqb. lyyalinata Germ: fufcata Germ. 6 punctara A. Ital. 2 fafciata Güll. gibha Eur. fafciata N. Holl. cinerea $N$. Holl. tropica $A m$. fugax $N$. Z $Z e l$. aurata Ind.'Or.' nitida $N$. Holl. rufipes $L$. Eur. 2 punctata Enr. nigricornis $N$. Holl. exaltata $E u r$. maculata Gerìs. tricolor Barb. guttata Ital. abdominalis BraJ. capenfis. nigrita Malab. turcica Braf. nigripes C.B.S. villofa Matab. hirta S. $N$. difcolor Barb. flava Ind. Or. bicolor N. Holl. 


\section{$P$ iezat}

SpHex. flavicornis Malab. fpeciofa.

fulvipennis Ind. nobilis Cajen. brevipennis Barb.

B) immaculata. ocellata $A f r$. aeq. ruficornis Awu. mer. 4 punctata $H i f p$. B) 6 punctata. grofla S. $N$. coerulea $A m$. mer. fellata Am. mer. 96. plumbea Hifp.

\section{LARRA.}

vefpiformis Ind. Or. erythroceph. C. B.S. cincta.

ichneumoniform. Hung. crafficornis $H i f$ p.

4 pufulata Tranqb. ungulata $\mathrm{S} . N$.

8. rufifrons $I n d$.

Tiphis.

fernorata Angl.

hiftrionica Chin.

flavipes. Bart.

maculata Geoing.

5 cincta Angl.

variegata Sibiv.

annulata Chin.

haemorrhoid. Am.

nigra.

ephippium Am. radula $N$. Holl. dorfata Corom.
TífHa. ruficornis Tranqb.

3 fafciara St. Crutx. 3 cincta $A m$. collaris Chin.

Morio Hifp. villora Hung. 19. pedeftris N. Holl. Scolia.

atrata Awr mer. nigrita $A f r$ aeq. 4 maculata Am. b.

B) fafciata. flavifrons Eur. auftr.

B) nigricornis. 6 maculata Ital. haemorrhoid. Gernn. rubiginofa Ind. Or. ruficornis Seneg. variegata Cajen. 2 maculata $A m$. $b$. ciliata Hifp. nurea Barb. ferruginea $N$. Holl. fcutellata Neap. radula Am. Sept. 4 fafciata S. $N$. tridens Ital. hortorum Hifp. unifafciata Barb. maura Barb. collaris Hifp. 5 fafciata Afr. aeq. 5 cincia Ital. 2 cincla $A m$. notata A. Ital. 
S c o L i A. verticalis $N$. Holl.

4 pufulata Malab.

5 penctata Germ.

I 2 guttata $H_{i} ; p$.

(nec 8 gntt.)

4 guttata Germ.

6 guttata Dan.

prisnı Grom.

4 pund:ata Ital.

6 cincta fin. mer. InS.

interrupta Ital.

fenilis Burb.

7 cincta $N$. Holl.

globularis C. B.S.

39. cylindrica Ital.

Chrysis. (Goldwefpe. La Guepe dorée.)

fplendida Trainqb.

fmaragdula sim. bor.

fefliva Guin.

calens Eur.

fcutellaris A. ltal.

oculata Tranqb.

lyncea Siesra Leon.

carnea Ital.

fulgida. Eur. $b$.

purpurata Germ.

ignita L. Eur.

bidentata L. Eurur. b.

integra Hisp.

fuccineta L.. Eur. .

gloriofa Barb.

lucidula Eur.

fervida Ital.

aenea Germ.

aurata L. Eur.
Chrysis. regia Germ.

cyanea L. Eur. . nitidula $A m$.

23. amethyftina $N$. Holl.

Thynnvs.

dentatus. N. Holl. emarginatus $N$. Fioll. abdominalis $A f r$ aeq.

4. integer N. Holl.

LEV CO I S Is. (Schenkelwerspe.)

gigas Gall.

dorfigera Ital.

atra S. $N$.

4. petiolata Tranqb.

BEMBEX.

fignata $A m$.

$V$ Vefpa $L$.

punclata Braf.

roftigta Eur.

Apis L.

olivacea Rarb.

glauca Tranqb.

lunata Tranqb.

carolina.

repanda Tranqb.

americann.

fpinofa A. Am. Inf.

lineata.

Ariata.

integra Ital.

6 fafciata Ind. Or.

3 dentata Eur auffr. interrupta Ind. Or.

17. ruficornis Hifp. 
Vespa. (W ejpe. La Guepe.) cincta Tranqb. affinis Chinz. ephippiun N. Holl. orientalis. 3 cincta $A m$. analis $C . B . S$. carolina. cornuta $L$. Afr. Crabro L. Eur. th ) vulgaris L. Eatr. germanica. faxonica. gallica. holfatica. rufa L. Eur. b. norvegica. bicolor Chin. maculata $L$. Am.b. arenaria $A m$. lineata $A m$. ftriata Cojen. macaenfis. carnatica S. $N$. carnifex Braf. Ianio Braf. $V$. canudenfis $L$. fchach $N$. Holl. anuularis Am. bor. fufcata Am. mier. humilis $N$. Holl. chinenfis. 5 cincta Chin. tepida $N$. Holl. aeftuans $A f r$. aeq. hh) Die Horniffe.
VESPA. calida $L$. Sierra Leon. marginella $A f r$. aeq. aurata Sirrr. Leon. haemorrhoid. C. B.S. albifrons $N$. Holl. ' concinna $N$. Holl. uncinata $A u$. B) 4 déns $L$. rufjpes $I n$. m. pacif. brunuea Tranqb. niarginalis $C . B . S$. Neftor S. $N$. oculata Ital. dorfalis $A m$. $b$. parietum $L$. ferripes $A m b$. 4 cincta Germ. 4 falciata $D a n$. 5 falciata Germ. muraria $I_{\text {. E Eur. }}$ emarginata Germ. fimplex Germ. parietina Germ. triloba Chin. fpinipes L. Eur: 6 fafciata Ital. bidens $L$. Eisr. $b$. radula Inf. Sandw. variegata $B a r b$. bifalciata L. Germ. 3 falciata Dan. finuata Germ. 3 punctata Barb. biglumis $L$. Eur. bicincta $C . B$. S . G 2
VEspa. 
Vesra, nidulans Cajen. tricolor Jamaic. biguttata Chin. aucta Girm. 2 punctata Germ. 4 punctata $7 x d$. flavipes $A m$. variabilis $N$. Goll. hebthes Ind. Or. cinera'cens $A m$. tecta Afr. arq. lateralis $C . B . S$. nigma Ind. Or. flavefcen: In 1. Or. Tamula S. $N$. gibbora Am. americana. coarctata L. Eur. arcuata $N$. Holl. cambaniformis $N$. Hoill.

guineenfis. cyanipenuis Cajer. petiulata Malab. conica Chrn. pyriformis Chin. pomiformis Ital. grifea Sierra Leon. cinerea Afr. apq. macilenta $A f r$ aeq. efuriens Ind. ferruginea Ind. Or. tabida Afr. aeq. phthifica Am. attenuata $A m$. cyanea Braf.
Vespa, varis Chiz. arietis $A m$. I05. pygmaea Cajen. Masaris. vefpiformis Barb. - apiformis Ital.

Mellinvs. myfaceus Eur. $b$. Sphex myjtacea $L$. fabulofus Dan. ruficornis Germ. 2 punctatus $D a n$. 3 cinctus ltal. campeftris Eur. Vespa'L. arvenfis Eatr. Vespa L.

8. 5 cirictus.

Philantus.

coronlatus Ital. auritus A. Ital. Triangulum $/ D a n$. Diadema C. B. S. rufipes $H i j p$. arenarius Eur.

Sphex $L$. ornatus Germ. flavipes Ital. 6 punctatus Ital. 5 cinctus Germ. lactus $H i f p$. trifrigatus $A m$. (Vefpa A.) interflinctus S. $N$. ruficornis Ital. diflectus. 
Philantvs. 5 maculatus Doiz.

7. ater Iral.

Crabro. (Le Frelon.) fpinofus Exur, ourfr. cornutus Ind. Or. folforius Eur. Sphex $L$. 4 maculatus Dan. repandus Ind. nitidulus Ind. Or. fibterraneus Dane. maculatus Ain. $b$. 6 cinctus Germ. interruptus Ind. Or. labiatus Germ. 4 cinchus Dan. pclatus Ewr. cribrarius Eur. Sphex $L$. clypeatus Germ. Sphex $L$. fcutatus Dan. vagus Fur. tridens Ital. dimidiatus Germi: pickus Germ. 2 cinctus Dan. 3 cinctus Am. uniglumis Eur. $V e \int p a L$. lineatus Germ. mucronatus Germ. 3 fpinofus Germ. leucoltoma Eur. $V$ efpa $L$. geuiculatus Gall.
4 punctatus Grill. lugubris $G$ rrnu. albilabris $G r m$. 32. minutus Dun is Hydaevs.

cylindricus Gersm. alternans Barb. 4 cinctus $\mathrm{Dan}$. maxillofus Eur. Apis L. florifomnis Eur. Apis 1. 6 cinctus $A$ in. thoracicus Ital. flavilabris Ital. truncorutn Eurr. Apis L. albilabris $B a w b$. flavipes $G_{s i}: m$. annulitus Eur. Apis $L$. albipes Ital. fimilis Dan. triangulum Braf. 16. Morio Gall. ANdRENa. (Ringbiene.) coerulefcens Eur. $b$. Apis $L$. rufipes $B a: b$. fpiralis Gall. cornuta Barb. labiata Germ. florea Germs. metallica Aur. G 3 ANDRE:

i) Yorro: Crabro V. flavum. P. 
ANdrena. aenea Eur. Apis $L$.

cyanea $B a r b$. marginata Germ. helvola Eur. Apis $L$.

bicolor Dan. tricolor Am. curvipes Tranqb. cincta Inu. Or. Atrigata $\mathrm{Ind}$. Or. nigrita $A m$. fafciata $A m$. zonata Ind. Apis $L$. circulata Guin. pilipes Paedeqn. Apis $L$.

carbonaria Germ. Apis $L$.

hirfura Hifp.

hirtipes Germ.

hasmorthoid. Surc. gulola C. B.S. bidentata Am. nigricornis Gieorg. virefcens Ain. cingulata $N$. Holl.

3r. fuccincta Eur. k) Apis L.

ApIs. (Biene. L'Abeille. Ber.)

latipes Chin.

Morio Braf. violacea Eur. aufir.

k) L'Abeille mineufe à sorcelet roux et velu.
Aris. feneftrata S. $N$.

Fplendida Cajen. nigrita Sierra Loon. dimidiata Sustin. carolina $L$. terreltris L. Eur. B) nitior. cryptatum $D$ an. ruderata Dan. nemorum Dan. foroenfis. lapponica. furinamenfis. virginica. antiguenfis. hifpanica. americanorum. caffra. africana. olivacea Sierra Leon. hortorum L. Eur. equeftris Eur. $b$. arbultorum Dan. Apis pratorum L. lapidaria $L$.

B) misor: rupentis Gevm. fylvarum $L$. Eur. Pryorum $N$. Holl. agrorusn Eur. aprica (nec rupeftis $\AA_{\text {. }}$ ) Lapp. italica. mnfcorun Eut. Hypnorum L. Eur.' ') , APIS.

1) Die Aftmoosbunumel. 
ApIs, fibirica.

nidularia S. $N$. pratorum Dan. lucorum $L$ Eur. acervorum $L$. Exur. fubterranea $L$. Eur. parietina Germ. Mniorun Gern. brafilianorum $A n$. aeftuans. L. N. Holl. tropica $L$.

autumnalis Germ. fervida S. $N$. elata S. $N$. fenilis Don. veterana Germ. pyginaea Gtrm. feminuda Germi. lagopoda $L$. Eur. hirtipes Surin. purpurafcens. amethyftina Ind. Or. amalthea Cajen. mufitans Cajpn. pilipes $A n g l_{0}{ }^{2 m}$ ) plumipes Ind. armata Suwin. canicularia $L$. Eurr. mellifica L. Eur. ${ }^{\mathrm{n}}$ ) cerana Chirz. indica S. $N$. guineenfis. disjundta $A m$. Inf. rufipes $A f r$ aeq.

m) Der Fedcrfu/s.

n) L'Abeille domeftique. A. des roches.
ApIs. thoracica Dan. dorfata Ind. Or. veffita Gall. flavifrons Braf. cineraria $L$. Eur. b. acraenfis Guin. analis Am. haemorrhoa Germ. barbara Bavb. nidulans Gall. manicata L. Eur. florentina.

Ireos Roff. ftictica Barb. maculata Ital. ${ }^{\circ}$ ): interrupta Ital. variegata $I t a l$. rotundata. Dan. fulvipes $H l \int_{p}$. globofus $A m$. mn. Inf. ferruginea $H i / p$. tuberculara Germ. fafciata $A f r$. bicornis L. Eur. tunenfis. rufä $L$ : Eur. rufipennis St. Crucis. lunata ' $A m$. $m$. bicolor In'd. Or. hirta Germ. holofericea Anu, Inf. villofa Ind. Or. pubefcens Ital. argentata Rarb. myllacea $N$. Holl.

G 4 ApIs.

o) Die gefleckte Biene. 
A Prs. centuncularis. $L$. Evr.

B) major. punctata Angl. luctuofa Ind. Or. 2 cincta Ind. Or. grifea A. Iral. mufciformis Guin. bombylans $N$. Holl. mulcaria $N$. Holl. haemorrhoid. Am. Inf. bucephala Guin. dentata $L$. cordata L. Surin. craflipes Am. mer. verficolor Ann. $\ln$ f. 4 dentata $L$. Eur. b. lanipes $A m$. Inf. coecutiens Germ. 3 dentata $A n$. ner. conica L. Eur. $N$. Holl.

fernicincta $N$. Cambr.

fpinipes $N$. Cambr. 4 cincta S. $N$. , labiata.

feladonia A. Ital. viridula.

aenea $\mathrm{S} . N$.

fmaragdula Tranqb. callola A. Ital. minuta Germ.

I 35. parvula (nec pygumaea A.) Ital. cincta Afr. aeq. Horea Ind. Or.

EVCEKA. lougicornis Eur. P) Apis $L_{\text {s. }}$ atricotuis $B a r b$. tumulorum Eur. Apis $L$. glauca $O r$. linguaria $G$ erm. grifea Barb.

7. antenuata Eur.

Nomada.

Hifrio Ind. Or:

fcutellaris Sibir.

punctata Canad.

tibialis.

variegata Eurs. Apis $L$.

agreftis Hifp.

ruficornis Eur. Apis $L$. rufipes Gern. ftriatz Eur.

Fabriciana Suec. Apis $L$.

fulvicornis Ital. gibba Angl. albilabris Germ. Haitorfiana Germ. I 5 . cingulata Suec. Form I A. (Anteife. La Fonrmi.)

herculeana L. Eur. Am. comprefla Tranqb.

ForMI-

Evcera.

p) A, à longues anten- 
Formic A. fmaragdina Ind. FOR M I A." caefpitum $L$. Ant.

rufipes Braf. bicolor Barb.

erythroceph. $N$. Hollo . didyma Ital. rufa $L$ Eur. Ams. pubefcens Hung. nigra $L$. Eitur. fufca $L$. Eur. ferruginea S. $N$. flavelcens Cajen. melanoceph. Cajerr. rubra $L$. Eur. cinerafcens Trangb. elongata Trangb. 6 guttata St. Crucis. foetens Guino. albipennis St. Crucis. rufibarbis Gall. obfoleta L. Eur. 4 punctata Gall. virefcens $N$. Holl. faccharivora $L$. Am. mer.

maculata Afr. aeq. barbara $L$. viatica Hifp. conica S. $N$. pallipes Cajen. aegyptiaca. antiguenfis. guinenfis. binodis Aegypt. omnivora L. Surin. flava Eur.

Evir.b.

tuberum Suec. vagans Germ. acervorum Dan. unifpinofa Guadel. grofla Cajan. auftralis N. Holl. bideus $L$. Stwrin. bifcutata Cajen. clavata $I n d$. attelaboides Braf. arenaria Barb. megacephala Isle de Franie.

Ammon N. Holl. bihamata St. Iohans. militaris Afr. aeq. 4 dens Cajen. cephalotes $A m$. mer. 6 dens L. Am. mer. atrata $L$. Am. mer. gulora $N$. Holl. forficata Terra Diem. roltrata Cajen. hainata $C a j e n$. haematoda. L. Am. mer. 62. maxillofa Ind. Doryevs. helvolus $C . R . S$. Mutilla $\boldsymbol{L}$.

Mvtrlea. (Afterbience. La Mitilla.) coccinea L. Ams. S. antiguenfis. guinenfis. 
Mv'trcla. Diudema Súrin. Mvticla. barbara. americana $L$. formicaria $N$. Holl. exulans Am. aurata $N$. Holl. europaea $L$. ruficornis $N$. Holl. atrata L. Afr.

B) alata. maura L. Eur. hungarica. coronata Germ. halenfis. arenaria $\mathrm{Hi} p$. ephrppium Germ. italica. pasdemnntana $A$. verficolot $A$ thn. regalis Hung. ruficollis Ital. ciliata G.run. erythrocephal. glabrata $O r$. rufipes Germ. melanoceph. Gall. vagans S. $N$. 30. nigripes $O r$. 


\section{$\longrightarrow+0+\infty=00+0$ \\ C L A S I S V.

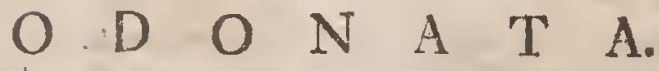

Palpi duo breves. Maxilla comea, dentata.

64. Libellvea. Labium trifidum: lacinia dorfali minutiflina.

165. AESHNA. Labium trifidum: laciniis aequalibus; lateralibus truncatis, dentatis, intermedia emarginata. I66. AGRION. Labium trifidum, laciniis' exterioribus bifidis.

\section{O D O N A T A.}

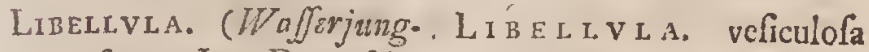
fer. La Deinoifelle. Am.

Dragon Fly.) 4 maculata L. Eur. confpurcata S. $N$. deprefla L. Eur. 3 maculata Carol. 2 fafciata $A m$. 4 punctata $A m$. flaveola L. Eutr.

B) innmaculata. lineata Ind.

Atgmatizans $N$. Holl. oculata $N$. Holl. indica.

L. Variegata L. Murcia Ind. Or. rubicunda $L$. Eur. .

afratica S. $N$. gibba S. $N$. vulgatillima $L$. Eur. obfcura Anu. vulgata $L$. Eur. finuata. cancellata $L$. Eur. carnatica S. $N$. braminea S. $N$. flavefcens S. $N$. pedemuntana. fafciata $L . I n d$. umbrata Anz. ns. fervata Chint. 
Libellyla. notata: Sierr. Aesha.

\section{Lions.}

dimidiata $A m$.

equeftris $A f r$. neq. Tillurga $S . N$. fludtuans $1 n d$. Or. nebulofa Ind. Or. chinenfis $r$. verficolor $A m$. vibrans. americana $L$. marginata Afr. ferruginea Chim. tomentola Am. acnea L. Eur. cyanea $A m$. 6 maculata Chin. contaminata lnd. Or. Eponina Carol. variegata $A f r$. aeq. carolina.

49. capenfis. forcipata Eur. ")

Libillula $L$. heros S. $N$. grandis Eur. Libellula $I_{-1}$ variegata Terra del Fuego. clavata Chin. annulata S. $N$. 7. minuta Chin. AGRION.

Virgo 'Etur. b) Sibellula $L$. puella Eur. Libellula $L$. coromaudelina S. N. ciliata Corom. nobilitata $A m$. mer. 6. linearis Ind.

a) La Caroline. b) La Louife. 


\section{CLASS IS VI.

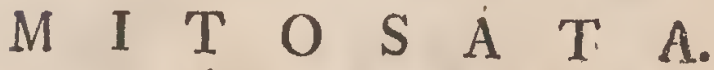

Palpi duo. Mraxilla filiformis, membranacea. 167. SCOL OPENDRA. Palpi, filiformes inter maxillas connati. Labium dentatum, fifum.

168. IVt.vs. Labiun crenatum, emarginatum. 169. ONiscus. Maxilla truncata, denticulata. Labium bifidum.

\section{M I T O S A T A.}

Scozopentra. (Sholopem. dBr. La Scolopendre.) lagura $L$. Eur. colcoptrata Eur. longicornis Tranqb. forficata $L$. Eur. Am. gigantea $L$.. Aws. morfitans $L$. Ind. Or. ferruginea $A f$ r . dorfalis Tranqb. clypeata Tranqb. electrica $L$. Eur. phofphorea $L$. Afiae. occidentalis $A m$. Gabrielis L. Ital. 14. marina $L$.

IvLvs. (Vielfußs. Le Gulle.) ovatus L. Oc. Eur. complanatus $L$. Eur. depreflus Ind. Or. ftigma Tranqb.
3 dentatus $A m$. varius ltal. craflus L. Afiae. terreftris L. Eur. carnifex Tranqb. indus $L$. fabulofus L. Eur. fufcus L. Ind. 13. maximus L. Am. Oniscus. (KellerafJel. Le Cloporte.) maculatus Ital. puftulatus Eur. Afellus $L .{ }^{2}$ ) fylveftris Giall. Armadillo Ent. 6. annulatus. ${ }^{b}$ )

a) Le Cloporte ordinaire. Die gemeine Ajfel.

b) Porro: Onifeus zonatus. P. et $O$. agilis $P$. 


\section{L A S S I S. VII. \\ $V=N \quad O \quad G \quad A \quad T \quad A$.}

Palpi duo porrecti., Maxilla cornea, unguiculata.

I7o. Trombidiva. Palpi incurvi, apice acutiffimi. Labium concavum maxillas recipieus.

I 7 I. Aranea. l'alpi incurvi maris clavati. Labium breve, apice rotundatum.

I72. Phalangivir. Palpi incurvi. Mandibula chelifera.

173. Tarantura. l'alpi porrecti, fpinofi, apice unguicnlati. Mandibula nulla.

174. Sconpto. Palpi cheliferi. Labium bifidum. 


\section{VII. $V \quad N \quad O \quad G \cdot A$ T A.}

TROMBIDIVM. tincłorium Guin. Acarnzs L. .

holofericeum Eur. Acarus L.

aquaticum Eur. Acariss $L$. caudatum Eivr. craflipes. unidulatum. maculatum. complanatum. verficolor. liliaceum. Torris. nodatum. ovale. truncatulum. frigatum. mufculus. integrator. finuator. tricufpidator. albator. pultulator Dan. variator. globator Eur. papillator Eur. fpinipes. vernale.
TR OM B I I V M. trifurcale Eur. ftcllare. ellipticuni Eur. orbiculatum. lugubre. geograplicum. abftergens. extendens. impreffum. 36. calcareum. A R A N A. (Spinne. L'A. raignée. Spider.)

* oculis :::: extenfa $L$. pallens A. Lim. Inf. lobata C. B.S. longimana lam. regia Tranqb. nobilis. virefiens Seland. cancriformis l. Am. venatoria $L$. Am. $m$. 13 guttata Ital. latens Angl. bicolor. fignata Germ. mactaus Am. cicurca Gern. bicornis Trangh. 
** oculis .:::

Aranea. uigrita Gern. glob: : Germ. *** oculis.." $\therefore$

horrida Germ. nuaxillofa St. Crucis. firaragdula Gall. latro Ans. viatica $L$. Eur. domeftica L. Eur. dorfata Germ. teftacea Am. mer. imprella Norte. laevipes L. Eur. tricufpidata Germ. ***** oculis: : : argentata ind. *** oculis $\therefore .:$. fafciata Madera. angulata $L$. diadema $L . E u r$. marmorea Eur. quadrata Eur. 6. liturata Gall. rapax S. $N$.

4 punctata L. Eur. militaris An. fpicsola $L$. $A m$. aculeata $A n$.

tetracautha $L$. $A m$. In $\int$. geminata S. $N$. hexacantha Ianaic. fornicata $N$. Holl. labyrinthica $R$. palunris L. Dan. aquatica L. Eur.
ARANEA. Obfcura Gall.

fcalaris Gall. 3 guttata Gall. fcorpiformis Gemm. 6. punctata L.' Eur. carnifex Angl. * * oculis clavipes $L$. Am." ". faccata L. Evir. fumigata $L$. $* * * * * *$ oculis $\vdots \vdots$ finbriata L. Eur. dorfalis Angl. fanguinolenta L. Eur. pupillata Chin. fcenica L. Eurr. ") fulvata Cajen. pubelcens Germ. cinerea ad litt. m. Balt. 3 lincata Nenp. Tarantula L. Ear.

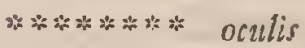
avicularia $L, A m$. mer.

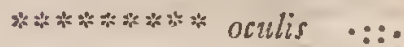
Taurus St. Dom. arcuata Ind. Or. maculata Chin. pilipes $I n \alpha$. $O r$. myopa Grim. longipes Aufrala. rufipes Márocco. fenoculata $L$. Germ. ARANEA.

a) I.'A. fauteufe, 
ARANEA. fcopulorum Norie.

lufca Tranqb.

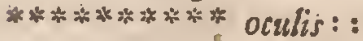

parallela A. Am. Iñ.

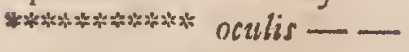

calceata Guin.

cruentata Braf.

fexcufpidata C. B.S.

hirtipes Cajen. tribulus $C . B . S$.

85. elegans Am.

Phalangivm. (Afterfpint ne. Le Faucheur.)

bicolor Helv.

Morio Norw.

Opilio L. Eur.

cornutum L. Eur.

bilineatum Norw.

Diadema Norw. carinatum Germ.

2 maculatum Angl. aranoides $C . B . S$. ')

b) Porro: Phalangium horvidum et Hellwigii. P.
Tarantula. reniformis $A m$. mer. Phalanginu $L$. caudata Ind. Or. Phalangium $L$.

3. lunata $A n$.

Scorpio. ( Scorpion, Lo

Scorpion.)

auftralafiae. maurus $L$. afer $L . I n d$. americanus $L$. europaeus. mucronatus S. $N$ : Tamulus S. $N$. hotrentottus. grifeus $A m$. $I n$ J. aufralis Afr. cimicoides Germ. cancroides Eur. ') Phalangium $L$.

13. acaroides $A m$. mer: Phalangium L.

c) Le Scorpion araignés. 


\section{L A S S I S VIII.}

\section{$\begin{array}{lllllllllllllllll}A & G & O & N & A & T & A\end{array}$}

Palpi faepius fex. Maxilla onunino anilla.

18r. Lrirvevs. Palpi poftici chelati. Antennae nullae.

183. Сумотнол. P'alpi et mandibulac mullae. Antennae quatuor, fefliles.

75. CanCer. Antennae quatuor filiformes: articulo ultimo bifido.

76. Pagvevs. Antennae quatuor pedunculatae: aniticae fetaceae, pofticae filiformes: articulo ultimo bifido.

179. Scrlzarvs. Antennae duae filiformes: articulo ultimo bifido. Squamae duae aculeatae loco pofticarum.

173. Hrypa. Antennae duae pedunculatae, fetaceae, pilis denfis ciliatae.

177. Galathea. Antenaae quatuor inaequales: anticae pedunculatae, fetaceae, pofticae feffiles filiformes: articulo ultimo fetaceo.

130. Astacvs. Antennae quatuor pedunculatae: anticae elongatae, fetaceae, pofticae fiflae.

184. Sevilia. Antennae quatuor fubaequales: anteriores pedunculatae, fetaceae, pofticae pedunculatae: pedunculo quadriarticulato, fetaceac, fifiae.

185. Gammarys. Aatennae quatuor pedunculatae, fimpliciflunac: anticae breves, fubulatae, pofticae fetaceae.

182. Monocvivs. Palpi quatuor: poftici auriformes. Antennae filiformes, ramofie, 


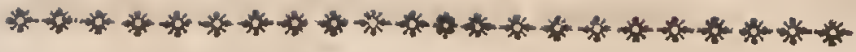

\section{A G O N $\dot{A} \mathrm{~T}$.}

Cancer. (Krebs. L' Ecreo Cancer. vocrars. L. Ia. viffe. (rab.)

* Thrvace latvi lateribus integerrinis.

raninus L. Mar. Ind. grapfus L. Am. mer. Inf.

mutus L. Mer. medit. cordatus L. Surin. quadratus Iamaic. ceratophthalmus m. Ind. ruricola $L$. Am. pifum I. m. Brit. $^{2}$. globofus litt. Malab. craniolaris L.litt. Malab. porcellanus Tranqueb. excifus $O_{c \ell a}$ anft. fagittarius Guadel. granarius Eur. minutus L. Pelag. pufillus Oc. bor. Pinuotheres $m$. Afrat. Pinnophylax L. Am。 orbiculus $N$. Zel. chelatus $N$. $Z$ el. longicornis $L_{\text {. }} O_{\varepsilon}$. Eutr. hexapus L. Oc. Eur.

* Thorace laevi lateribus incifiss.

coralliuns Inds

tioridus L. Lind.

maic. ")

planatus Tesr. d. Fuego. 2 fpinofus Ind. Or. rhomboides L. mar. mid, maculatus Lo mar. Afiat. fanguineus Pelagi. pelagicus. defenfor $O c$. Auffr. haftatus Am. Inz: armiger $O \varepsilon$, auftr. gladiator N. Holl. forceps $O t$.

victor litt. Malab. angulatus $O c$. Angl. marmoratus. varicgatus Am. mer. Int. maenas L. Oc. Eur. Af. pygmaeus $O c$. Angl. parvulus Am. m. $\ln$. depurator L. Oc. Eur. dromia $L$. Ind. Or. fexdentatus Int. Or.

- feriatus L. Mar. Ind. lancifer $O c$. pacif. pagurus L. Oc. Entr. th Ind. b)

$\mathrm{H} 2$

CAN:

a) The land crab.

b) Der Tugttenkerebs. The punger, 
Cancer. fornicatus Ind. Cancer. longiroftris Mar.

Or.

I I dentatus Am. bor. granulatus Mar. med. tuberculatus $O c$. pacif. Calappa L. Oc. anuer. fpinifrons.

\section{aeneus L. Ind .}

depreffus Mar. medit.

am.

ochiodes Tud. or.

* * Thorace Jupra hirto et Spinsojo.

aegagropila $O c$. auftr. cylindricus Trangb. chabrus $L . O c$. lnd. lanatus L. Mar. med. mafcaronius $M a r$. med. nafutus $O c$. Norweg. urfus $O c$. auftr. hircus Iamaic. Araneus L. Oc. Eur. opilio Mar. medit. condyliatus Mar. ned. ovis Ind. Or. muricatus $1 n d . O r$. roftratus L. Oc. Eur. tribulus L. Mar. med. criftatus $L: O c$.

Eriuaceus $O c$. Ind. barbatus $O_{e}$. Neap. fuperciliofus $L$. Ind. $O r$ r punctatus L. $A f$. $A m$. nucleus $L$. Mar. qued. cornutus L. Mar. need. 7 fpinofus Oc. Ind. med.

fcorpio Oc. Norze. phalangium $O c$. vefpertilio Ind. puber Mar. medit. holofericeus $N$. Holl. quadridens $1 n d$. Or. aculeatus $O_{c}$. Norw. maja L. Oc. Norw. mammillaris Chiš. horridus L. Oc. Afiat. chiragra Mar. msed. gonagra Ianlaica. nodulofus. longimanus L. Oc. Afiat. longipes L. Oc. Ind. hyftrix $O c$. Ind. fpinifer $L . A$. Lar Ind. Or. germanus $L$. 102. auritus Island. PagvRvs.

(Cancer L.)

Latro L. Ind. Or. aniculus $O c$. areftr. Bernhardus L. Oc. Ent. hungarus Ind. Or. Diogenes L. Oc. Amer. miles Ind. Or. clypeatus $I n d$. Or: eremita Mar. Ital. tubularis L. Mar, med. oculatus $O c$. alatus Island. I 2.aranciformis Mar. Edimb. 
Galathea.

(Cancer. L.)

Arigola L. Oc. Eur. rugofa Mar. med. gregaria Oc. Amer. amplectens $O c$. atlant.

Hipsi.

- (Caneer L.)

adactyla $O c$. auftr.

fymnifte L. Ind.

fcutellata.

dentata Or. Eur. Irod.

dorfipes $L$. Oc. Ind.

fcabra $O c$. auftr.

7. variolola Oc. Ind.

SCYLLARYS.

(Cancer L.)

arctus. $L$. Oc. Eur. $A$ f.

Afr. Am.

auftralis. .

Astäcvs:

(Cancer L.)

marinus Oc. Norw. ')

C. Gammarus $L$.

fluviatilis Eur. ${ }^{\text {) }}$ )

Cane. Aftacus $L$. homarus L. Mar. $A$ S. Amer.

élephas Am. m. Inf. narval Mar. med. carcinus L. Amer. fulvus $O c$. coerulefcens $P$ elag.

e) Der Hummer. L'homard. 'The lobiter.

d) Dor Fujsktritss. L'ecreviffe, the craw - fifh.
Astacvs. fulgens $O$ c.

homari Oc. Norw.

Harengum $O \varepsilon$.

crafficornis Oc. Am.

hiftrio Groenl.

planatus.

cylindrus $O$ c: Ind.

malabaricus.

carinatus.

boreas $O c$. bor.

groenlandicus.

varius $O c$. Noriw.

emcritus $L . m$. Ind.

norwegicus $L$.

fquilla $L . E u r .{ }^{\circ}$ )

locufta $L$. Oc.

ferratus $O c$. Norre.

Crangon L. mar. Balt. ${ }^{f}$ )

27. tettigonus Island.

Limvivs.

polyphemus Ind.

Monoculus L. ${ }^{\text {g) }}$

cyclops Ind.

Monocvuvs. (Kiefenfu/s.

Le Minonocle.)

* tefta univalvi, oculis duobus.

Apus L. Eur. ")

H 3

Mono-

e) Die See-Garnecle. La Cherrette, Crevette, le barbot.

f) Caramote.

g) Der moluckifcbe Krelos The horfe floce, helmed Fifh.

h) Lc Binocle à queue en filets. 
Moxoculvs. pifcinus L. $O$ c. Argulus.

falmoneus $O c$.

* Tefta univalvi, oculo unico.

Satyrus Aq. pur.

Silenus $F o l$ :

Faunus Palud.

Pacchus Rip.

bracteatus Aq. pur.

faltatorius.

* Tefta bivalui, oculo anico antenzis ramo. fir.

Pulex L.

longifpiuus Aq. pur. quadrangulus $A q$. Angr. laevis Palud. rectiroftris Palud. curviroftris $A q$. tamf. 2 fpinofus Paiud. cryftallinus $A q$. pur. fetiferus $A q$. Jtagn. * Tefta bivalvi, oculo unica antenmis fmplicibus.

viridis Fuscor. Conferv. luteus Fucor. flavidus Finflr. gibbus Vlvar. coarctatus Fucor.

** Tefa bivalvi, octha lo unico, antennis api. ce floccofis. detectus Conferu. ormatus Aq. fongn.
Monocvuvs. laevigatus Palud. falciatus. nrigatus Aq. lim. viduatus Aq. lim. conchaceus $L .{ }^{i}$ ) pilofus. monachus Palud. craffus.

candidus Palud:

******* Tefta bivalui, oculis disobus, antersgis capillaceis. brachyurus Pahed. fpluaericus Lemnarum. quadrangularis Aq./lagm. lamellatus Aq. Jagn. trigonellus Palud. truncatus.

longiroftris.

macrourus in Lacubus.' is focors.

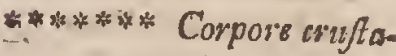
ceo, oculo zunico, ans tenнis fimplicibus. minutus Aq. Atagn. coeruleus Aq. ftagn. rubens $p$ alud. claviger. quadricornis $L .{ }^{k}$ ) crafficornis Palud. : chelifer Maris.

Mana:

i) Le Monocle à coquille longue.

k) Le Monocle à queus fourchue. 


$$
A g \circ n \text { a } t \text { a. }
$$

Monocvivs. longicornis Cymothoa. ceti Oc. bor.

Mar. Norte.

captivus Aq. Mar.

************ Tefta rullla,

$$
\text { oculo unico. }
$$

54. Pediculus L. Palud.

Crmothon.

paradoxa Terra d.Fuego. imbricata $N$. Zel.

falcata Oc. chinenf.

$\Lambda$ filus Oc. Eur.

Onifcus $L$.

Guadeloupenfis.

Oeftrum Oc. Ezzro

Onifcus $L$.

Entomon Oc Eur.

Onifcus $L$.

aquatica Eur.

Onifcils $L$.

njarinus Oe. Norw.

Onifcus $L$.

linearis Oc. Ind.

Onifcus $L$.

chelipes Mar. Atlant.

bicaudata Oc. Norw.

Onifcus $L$.

fcopulorum Oc. Norte.

Onifcus $L$.

americana.

piora Oc. Norte.

Onifcus $L$.

phyfodes Oc. Eur.

Onifcus L.

fpinofa Oc. Atlant.

acuminata $O c$.

cmarginata $O c$.

albicornis Mar. Hifp.
Onifcus $L$.

occanica.

Onifcus $\boldsymbol{L}$.

ferrata O $i$. Hijp.

24. affimilis $O c$. Equ

Onifcus $L$.

Sevilta.

(Cancer L.)

maculata Ind. Or.

mantis E. Mar. Af. Ind.

med.

fcyllarus $L$.

ciliata Oc. Ind.

chiragra Oc. auftr.

vitrea Oc. Atlant.

Gammarvs.

(Cancer L.)

ampulla Oc. Bor.

nugax Oc. Bor.

carinatus.

cancellus Sibir.

longicornis Oc. Eur.

C. grofipes $L$.

loculta $L$. Eur.

pulex L. Mar. ${ }^{1}$ )

corniger Oc. Norw. linearis L. Oc. Isl.

falinus $L$. Angl.

ftagnalis $L$. Eurr.

gibhofus Oc. Liv.

efca Oc. Norw.

I.4. Medufarum Oc. Norwe. $\mathrm{H} 4$

AGO.

1) Der Flobkrebs. La Crevette des ruiffeaux. 


\section{AGONATA SEC. DALDORFIVM.}

AlpheVs.

(Cancer $F$.)

* corpore gibbo lateribus inaequalibus.

Maenas.

Pagulus.

tinctor S.N.

pictor S.N.

Rumphii S.N.

feaber S. N.

parvulus.

Gonagra.

ochtodes.

bifpinofus.

coralliuus,

Vefpertilio.

fetolirs S.N.

* obcordati corpore gib. bo lateribus laevibus antice tantun carina. tis.

ruricola.

fenex S.N.

B) vitulus,

maculatus.

3 dens S.N.

vocans.

augulatus.

*** quadrati corpore gibbo

Jupra plano marg. un. dique carinato.

tetragonus S. N. quadratus S.N. rhomboides.
* *** deprefi corpore de. preflo plano.

Alphevs. "grapfus. variegatus. litteratus S. N. depreflus. pifum.

minutus. pufillus.

* $x$ obverficthor. inte. gro poftice latiore. femicylindrus S. N. fexpes S.N. auritus.

OCYPODE.

(Cancer F.)

ceratophthalma. quadrata. rhombea S. N.

Calappa.

(Cancer F.)

fornicata. tuberculata. granulata. fpinifrons. marmorata. Parthenope.

(Cancer $F_{i}$ )

fornicata.

Giraffa S.N.

longimana. regina S. N, lar S.N. dubia S.N. 
Leveosia.

(Cancer F.)

- chelis ovutis. fcabriufcula S. N. pila S. N. globofa. graniolaris. porcellana. planata.

* manibus filiformibus. punctata. fugax S.N. Nucleus. 7 fpinofa.

B) C. Hyftrix F.

Erinaceus. sylindrus.

Matvia.

(Cancer F.)

victor.

planipes S.N.

SyMETHis.

variolufa.

(Hippa F.).

Ligia.

(Cancer $F$.)

inflexa $\mathrm{S}$.

3 cufpitata.

granaria.

DROMIA.

(Cancer F.)

Rumphii (C. Dromia

F.)

artificiofa S. N. auftralafiae.

(C. aegagropila F.)
B) Caput mortuum L.? Portvnvs.

\section{(Cancer F.)}

* thor. utr. bid. th. utr. 5 dent. vigil S.N. puber. depurator. feriatus.

\section{Lancifer.}

* thor. utr. 5 desut.

Crucifer. S.N. Lucifer S. N. annulatus $S, N$. variegatus $S . N$. B) affinis. hololericeus. truncatus S.N. . ** * th. utr. 9 dent. aequal. tranquebaricus S.N. **** thor. utr. 9 dent. - dente poftico majore. pelagicus. fanguinolentus S. N. defenfor. haftatus. gladiator S. N. haltatoides S. N. forceps. ponticus S.N.

INACH v S.
(Cancer F.)

- pedibus cylindricis mw dioribus. muricatus. hybridus S.N. 
INACHVS.

Ovis.

- Hircus.

cornutus.

Araneus.

aculeatus.

Chiragra.

nafutus.

anguftatus S.N.

* pedibus elongatis fili. formibus.

Scorpio.

Phalangium.

longitoris.

Lar.

B) longipes $F$.

fagittarius.

Doripre.

(Cancer F.)

quadridens.

B) C. lanatus F.? aftuta (C. Pinnophylax F.?)

callida(C.Pinnotheres F.?)

Orithvia.

mammillaris.

(Cancer $\mathrm{F}$.)

Pagvevi:

Latro.

aniculus.

Bernhardus.

hungarus.

Diogenes.

miles.

ophtalmic. S. N.

cultos S.N.
PAgvrvs. clypeatus. diaphanus S. N. eremita. tubularis. oculatus. alatus. araneiform.

Galathea.

Arigofa. rugola. gregaria. amplectens.

IDoteA.

adactila (Hippa adact.F.) armigera (Áltac. emeri. tus F.)

ALBVNEA.

(Hippa $F_{0}$ )

fymnilta. dorfipes. fcabra.

Evryala. dentata (Hippa F.)

SCYLlarVS.

Aretus. aequinoctialis $\mathrm{S} . \mathrm{N}$. antarcticus S.jN. orientalis S. N. anftralis.

Pallinvrva.

\section{(Aftacus $F$. )}

Homarus S.N. ornatus S.N. falciatus S. N. quadricoruis (A. elephas F.) 
Palaemon.

(Aftacus E.)

carcinus.

Lar S. N.

longinan. S. N.

brevimanus S. N.

(Aftacus F.)

coromandelianus S. N. tranquebaricus S.N.

Squilla.

ferratus.

Fucorum S. N.

Penevs.

(Aftacus $F$ )

Monodon S. N.

monocerus S. N.

planicornis S. N.

Homarvs.

(Aftacus $F$.)

* chelis 6.

marinus.

fluviatilis.

Bartonii S.N.

* chelis 4.

norvagicus.

B) Ininor.

faber S.N.

fulvus.

Crangon.

(Aftacus $F$.)

avarus $S . N$.

Tamulus S. N.

rapax S. N.

nualabaricus.

PUSYDON.

(Ajlacus F.)
Posydon. depreffus S. N. cylindrus.

Limvers.

Polyphemus.:

Cyclops.

Monocvevs.

* teffa univalui ocul. 2. apus.

pifcinus.

argulus.

falmoneus.

* * tefta univalvi ocul. x.

Satyrus.

Silenus.

Faunus.

Bachus.

bracteatus.'

'faltatorius.

* * * tefta bivalvi oculo I! anten. ramojis.,

Pulex.

longifpinus:

quadrangulus.

laevis.

5. rectiroftris.

curviroftris.'

2 fpinofus.

cryftallinus.

fetivorus.

*** tefta 2. valvi aculo

1. ant. fimpl.

viridis.

luteus.

flavidus.

gibbus.

coarctatus. 
**** tefta 2 valvi oculo

I. ant. ap. floctofis.

Monocvivs. detectus. ornatus. .

laevigatus.

fafciatus.

frigatus.

viduatus.

congaceus.

pilofus.

monachus.

craffus.

candidus.

****** tefta 2 valui oc. 2. ant. copillaceis.

brachyurus.

fphaericus.

quadrangular.

lamellatus.

trigonellus.:

truncatus.

longirofris:

macrourus.

focors.

******* Corp. cruftaceo

$$
\text { oc. I. asut. fimpl. }
$$

minutus.

coeruleus.

rubens.

claviger.:

4 cornis.

crafticornis.

chelifer.

longicorn is.

* * * * * * * * * teft, null, oc, I. captivus.
Monocvlvs. pediculus.

Cymothos.

paradoxa.

imbricata.

falcata.

Afilus.

guadeloupenf.

Oeftrum.

Entomon.

aquatica.

marina.

linearis.

clielipes.

B) deprefia.

bicaudata.

fcopulorum.

americana.

Pfora.

phylodes.

ipinola.

acuminata.

emarginata.

albicornis.

Cethi.

oceanica.

ferrata.

affimilis.

Sevizla.

maculata.

Mantis.

Scyllarus.'

Raphidia S.N.

Phalangium S.N.

Ichneumon S. N.

ciliata.

chiragra. 
Sevilla. vitrea.

Gammarvs.

anpulla.

nugax.

carinatus.

cancellus.

longicornis:

Locufta.

Pulex.
Gammarvs. corniger. linearis.

B) Cancer atomos $E$.

falinus.

nagnalis.

gibbofus.

Efca.

Medufarum.

Homari (Af. F.)

\section{CANCROIDEA DVBIA.}

Cancer.

raninus.

moutus.

cordatus.

excifus.

orbiculus.

chelatus.

longicornis.

floridus.

fanguineus:

pygmaeus.

II dentatus.

aeneus.

chabrus.

mafcaronius:

urfus.

opilio.

condyliatus.

roftratus.

tribulus.

criftatus.

barbatus.

fuperciliofus.
Cancer. Maja.

horridus. nodulofus.

longipes.

fpinifer.

germanus:

Hippa.

fcutellata. Astacys.

Narval. coerulefcens.

fulgens.

Harengum.

crafficornis.

Hiftrio.

planatus.

carinatus.

Boreas.

groenlandic.

varius.

Locufta.

Crangon.

tettigonus. 


\section{L A S S IS. IX. \\ G $\quad L \quad O \quad S \quad S \quad A \quad T A$.}

Lingua Spiralis inter palpos reflexos, fuppofos. 186. Papilto. Palpi duo reflexi. Lingua fpiralis exferta. Antenaae extrorfum craffiores.

187. HESPE:i IA. Palpi duo bafi compreffi, hirti, apice cylindrici, nudi. Antennae clava oblonga, faepius uncinata.

188. Sphinx. Palpi duo reflexi, pilofi. Lingua fpira. lis exferta. Antennae fquamatae.

189. Sesra. Palpi duo reflexi. Lingua exferta, truncata. Antennae cylindricae.

190. ZYGAENA. l'alpi duo reflexi. Lingua exferta, fetacea. Antennae medio crafliores.

I91. Bombyx. Palpi duo compreffi, reflexi. Lingua brevis, membranacea. Antenuze filiformes.

192. Cossvs. Palpi duo brevifimi, cylindrici. Lingua nulla. Antennac breves, filiformes.

193. Heprarvs. Palpi duo pilofi. Rudimentum lin. guae bifidae inter palpos. Antenuae moniliformes.

194. Noctva, l'alpi compreffi, pilofi, apice cylindrici, nudi. Lingua porrecta, cornea. Antennac fetaceae.

195. Hyblacai. Palpi porrecti, compreffi, medio dilatati. Labium porrectum. Antennae letaceae:

196 Phalaena. Palpi cylindrici. Lingua porrecta, membranaces, Auteninac filiformes.

197. Pyralis. Palpi nudiusculi, bafi cylindrici, medio dilatato ovati, apice fubulati.

198. Tinea. Palpi quatuor inaequales. Antennae fetaceae.

199. Alvcita. Palpi duo ad medium bifidi: lacinia interiore acutiffima. Antennae fetaceas.

200. Pterophorvs. Palpi lineaires. Lingua exferta, membranacea. Autennae fetacese.

IX. GLOS. 


\section{G L O S S A T A.}

PA PI I I O. (Taguogel. Papilio. $\beta$ ) Alcenor C. Schmetterling. Papil. Lyfander Ind. lon. Butterfly.)

- Equites Trojani. Paris L. Ajiae. Bianor Chin. Helenus $L$. $A$ S. B) Severus ,C. Thefeus Sumatra: Polytes L.Af. B) Alphenor $C$. Mutius Trangb. Heckor L. Af. Afcanius Braf: Antenor Ind. Troilus L. Anz. b: Glaucus L. Anr. Palinurus Tranqb. Crino Afr. Deiphobus L. Af. Pelaus Ind. Afterias Amer. Phorbanta Cajen. Philenor Amer. Cyrus. Pammon L. Af: Afus Am. mer. Acamas Iamaic. . Laodocus Braf. Achates Chin.

Polydorus I. Ind. or.

Coon Chin.

Antiphus Ind.

Dardanus Braf. Tros Braf.

Antimachus $A f r$.

Priamus L. Amboin. Amphrifus Ind. Remus Amboin. Laomedon Chirt. Memnon L. Chiv: B) Laomedon $\mathrm{C}$. y) Androgeus C. Aftyanax Ind. Protenor Surin. Agenor L. Chin. Anchifes L. Am. Sarpedon L. ASS. Polydanas $L$. Androgeus Susvin. Peranthus Coih. Chirr. Amphimedon Anuboin. Zacynthus Braf. Dimas Braf.

B) Hyppafon C. Idaeus Madras. Vertumnus Sturin. Aeneas Lo lnd. 
Parilio. Ilus $A m$. Iphidamas.

Belus Strin.

B) Lycidas C.

Pompejus Surin.

Polyinneftor $A$ f.

Panthous L. Ind.

Pandarus $L$. Ind.

Aftenous C.B.S.

B) Minos C.

Helena L. Am.

Heliacon Ind. Or.

Euryphylius $I$. Ind.

Creffida $N$. Holl.

Harmonia N. Holl.

* Equites Achivi.

Ripheus Corom.

Leilus $L$. Am.

B) Sloaneus C.

Lavinia Am.

Brutus Afr. aeq.

B) ecaudatus.

Dolicaon Am. mer.

Vlyfies $L . A$.

Diomedes L. Ind.

Pròtefilaus L. Am. S.

Antilochus L. Am.f.

Podalirivis L. Eur. ")

Antiphates Am.

Alcibiades Tranqb.

Pompilius Ind.

Sinon Ind.

Agapenor $A f r$.

Thyattes Braf.

Chiron Ind.

a) Der Secgelfoliter, Dis Regirza, Le Flambé.
Papilio. Crethon Surin.

Orfilochus Surin.

Curius Sicm.

Odtavius Suris.

Licarfis Surin:

Meliboeus Surrin.

Homerus Am.

'Turnus $A$ in.

Machaon L. Eur. ')

Terfices Am.

Codrus Amboin.

Chalcas Amer.

Meneftheus Ind.

Xuthus Ind. Or.

Terfander Sierra Leon.

Thoas L. Am. m.

Crefphontes Ind. Or.

Polycaon Surin.

Ajax L. Am. $b$.

Agamemnon L. Af.

Oreftes Afs.

Pylades $A f r$.

Demoleus L. Ind. Or.

Epius Chinz.

Leonidas Afr.

Tynderaeus.

Antheus Amboin.

Nireus L. Ind.

Lacedemon Malab.

Zenobia Sirrr. Leon.

Cynorta.

Papto

b) Der Scbroalbenfelbwanz. Der Königinnenpage. Le Grand Portequeue. Papillon page de la reine. Le Grand Pap. du. fenouil. 
PAPILIOC. Amphinachus Ind.

Amphitrion Am.

B) Canbrifius $C$.

Hippocoon Sierr. Leon.

diffimulis $L$. $A$ f.

affrmilis $L$. Chin.

** * Feftivi.

Cyparifias Am.m.

Midamus L. Ind. Or.

Dryafis.

Diocletianus Ind. Or.

Claudius Tranqb.

Niavius L. Sierra Leon.

Damocles Af. Afr.

Corus Ind. Or.

Tulliolus Ind.

Sylvefter.

Cochrus.

Liberius $A m b$.

Radamanthus $A$.

Zoilus $N$. Holl.

Cyntheus Afr.

Arethufus Surin.

Clymenus Braf.

Sifamnus.

Laflus Cajen.

Antiochus L. Chin.

Syphax Ind.

Evalthe Ind.

Licas Surin.

Phalaris.

Pelasgus Strin.

Phacton Am.

Sophus.

Galanthis Surin.
PAPIIO. Cenaeus $L$.

lnd.

Chermes $A f r$.

Hefperus.

Mirus.

Tytius Surin.

Eupalus Afr. aeq.

Pinthacus L. Ind.

Archippus Am. m.

Plexippus L. Aw.

Erippus Chin.

Mifippus L. Am.m.

Chryfippus L. Ind. Or.

Alcippus Am.

Eleus Am.

Erefimus Guia.

Diocippus Ind.

Gilippus Am. mer.

Hegefippus Sumatra.

Darius Braf.

Daedalus Guin.

Nicaeus Ind.

Numilius Braf.

Codomannus Braf.

Polymenus Suritu.

Hydafpes Braf.

Iairus $I n d .0$.

Haquinus Tranqb.

Orion Surin.

Cocytes Siam.

Drufius Inf. Rotterd.

Thymetus.

Abfolon Guin.

Odin Chin.

Delila Am.

Dido L. Am. 
Papilio. Eurinome $I n d$. Or.

Philomela Ind. fimilis Ind.

B) Aventina C. affinis $N$. Holl. Lais Ind. Or. Enothrea Sierra Leon. Dirtea Beng. Hippia Poulicand. Panope L. Ind. Iacintha Ind. Or. Micalia Ass. $b$. Palliflome Am. P. Vanillae L. Iuno Surivz. ***** Nymphales. Iafius L. Afr. Barb. Pyrrhus L. Ind.

Camillus Afr. aeq.

Sempronius.

Tiridates Amb.

Caftor Guin. Pollux Guino. Aeclus Amb. Paullus lan. Etheocles Sierr.'Leon. Fabius Ind.

Horatius.

Eudoxus $A f r$.

Cocles Sian.

Miltiades.

Varanes Int.

Themiftocles.

Periander Ind. Or. Lycurgus $A f r$.
Papilio. Decius Guirn.

Sabinus Anb.

Doreus Sicrra Leon.

Palamedes Am.

Orontes 1. Ind.

Aegifus Ind.

Phalaena Lanus $L$.

Solon.

Empedocles Ind. Or.

Euryalus Ax:boin.

Nifus Amboin.

Phidippus L. Ind.

Xiphares Afr.

Aurelins Ind.

Bernardus Chin.

Gerdrudtus.

Chorinaeus Surin.

Laertes Simin.

Morvus Ind.

Octavius Ind.

Eribotes Ind.

Itis Surin.

Cymodoce Surin.

Bifaltide Surin.

Arfinoe Amb.

Panthera Tranqb.

Rohria Ind.

Rebecca Guia.

Erota Afr.

Europa $A m$.

Acheronta Braf.

Troglodyta Ams.

Tetys Amu. m.

Portia Am. Inn. interrogationis $\mathrm{S} . N$.

C. aureum L. Af. 
Papilio. Ifidore Ind.

Jella $I n d$.

Furcula Ind.

Eurocilia Ind.

Phorcyps Ind.

Chelys Ind.

Lethe Ind.

Aftipa St. Thom.

Fatima lnd.

Achilles L. Am.

Medon L. Ind.

Erithonius Afr.

Ceres Sierr. Leon.

Vrfula $A m$.

Cato Sierr. Leon.

Philoctetes L. $1 n d$.

Menetho Ind.

Lucretius Guin.

Epiftrophus (nec Laertes)

An. m.

Stelenes L. Am. m.

Gambrifius Ind. Or.

Demophon L. Cajenz.

Neflor L. Am.

Perfeus Surin.

Ariflides Ind.

Telemachus Am. m.

Menelaus $I$. Am. $m$.

B) Rhetenor C.?

y) Adonis C.?

Teucer $L$. Am.

Automedon Srrin.

Hecuba L. Cajen.

Metellus Surin.

Idomeneus Is. Am.

m.

B) Illioneus $C$.
Papilio." Io L. Eur. ')

Polynice Sumatr.

Alumana L. Af.

Alerie $L$. Ind.

Oenone L. $A$ f.

B) minor.

Hierta S. $N$.

Flirtea Ind.

Lemonias Ind. Or.

B) Ononis I.

Vellida $\operatorname{In} \int . \operatorname{Am} /$.

Orythia L. Iamaic.

B) Lavinia C.

Clelia Guin.

Penelea Surin.

Zelima N. Holl.

Terea Sirrr. Leon.

Laodice Guin.

Lampetia L. Ind. Or.

Glyzerie Ind. Or.

Megaera L. Eur.

Aegeria L. Eur. ')

Xiphia Madera.

Minerva $A m$.

B) Arcadia C.

Ocypete Surin.

Ocirrhoe Sawin.

Penelope Am.

Aranea Surin.

Atlita Ind. Or.

I 2

PAP1-

c) Der Pfauentagfalter. Le Paon da Jour. The Pca. kocks Eye.

d) Der Queckenfalter. Der Waldargus. Le'Tircis. The Enfield-Eye. 
P A I I I . Iatrophae Am. mer.

Laomedia Ind. Or..

Libye Ind.

Hędonia L. Af.

Tullia Chin.

Merope $N$. Holl.

Jole Guin.

Hefione Surin.

Egaea Am.

Serina Ianaic.

Poltuerta Am.

Mylitta Ind.

Artemifia Ans.

Dorimene (nec Doris)

Surin.

Ianthe Cajen.

Laothoe Surin.

Gonerilla $N$. Zel.

Irea $N . Z$ Zl.

Portlandia Am. m.

Cardui L. Eur. Afr. ' )

Huntera $A m$.

Allionia Lufit.

Tulbaghia L. C. B. S.

Pipleis L. Ind.

Claudia Ind.

Blomfildia Braf.

Bankia N. Holl.

Solandra Otaheit.

Tolydecta Tranqb.

Tolumnia $l n d . O r$.

Morna Tnd.

Iuliana $A w b$.

Leda L. Sierr. Leon.

e) Der Difelfoirer. La bel.

le Donne, Painted Lady.
Papilio. Lena $L$.

Cajen.

Dydimene Surin.

Luna Surin.

Iphita Chin.

Callifo $A f r$.

Iris L. Eur. ' )

Jlia Germ.

Beroc Germ.

Avia Afr. agq.

Populi L. Eur. s)

Chryfippe N. Holl.

Cydippe $L$. Ind.

Marica Afr.

Althea Guin.

Amphiceda Guin.

Penthefilea Ind.

B) Biblis C.

Berenice Sierr. Leon.

Semire Sierr. Leon.

Cyane Ind.

Vitellia $A m b$.

Zenobia Sierr. Leon.

Antiopa L. Eur. Am. ${ }^{\mathrm{h}}$ )

Cacta Ind.

Pythia Guin.

Zingha Sierr. Leon.

Protogenia Iava.

Ameltris Ind.

Lamina Ind.

\section{PAPI.}

f) Der Scbillerfniter. Le Changeant,

g) Der Eisvogel. Der Afpenfalter. Le Silvain.

h) Der Tratersnantel. Le Mainteau bigarré. 


\section{$G l 0 \iint a t a$.}

PAPIL10. Atalanta L.

$$
\text { Eur. Af. Am. }{ }^{\mathrm{i}} \text { ) }
$$

Archefia lava.

Charonia Ind.

Biblis Am.

Hyperia Ind.

Prolope N. Holl.

Echo Surin.

OCtavia Sierra Leon.

Lubentina Chin.

Janaflia Afr.

polychloros L Eur. ")

V. album Germ.

Vrticae L. Eur. ${ }^{\text {') }}$ )

Clytemneftra Surin.

Dirce Ind.

Ifis Ind.

Oifis Ind.

Progne Am. m.

C. album $L$. Eur. ${ }^{m}$ )

triangulum Ital.

Merione Surin.

Ariadue $L$ Iava.

Bolina L. Ind. Or.

Liria Ind. Or.

Lafinafla $A$ f.

Clytia L. Ind.

Cocyta Ind.Or.

i) Der Admiralfaleer. Der Heiterneffelfalter. Der Admiral. Der Vulkan. Der Scbeckflitgel. Le Voulcain.

k) Dor grofse Fucbs.

1) Der kleine Fuchs.

m) Le Gamina. Robert Ic Diable. The Silyer Camma.
PAPILIO. undularis : ais

Corom.

Monina Ind. Or.

Hiarba Ind.

Amathea Cajen. Surin.

Meleagris Afr. aeq.

Melantha Guin.

Leucothoe L. Af.

Medea Surin.

Blandina Ind.

Amalia Sierra Leon.

Hoiftilia Sierra Leon.

Rhea M.

Nauplia L. Am. m.

Heliodore Siam.

Ophione Grinz.

Ilithuia Ind. Or.

B) Polenice C.

Amphinome L. Am.m.

Opis Sierra Leon.

Crithea Sierra Leori.

Phegea Ind.

Salmatis Afr.

Pelea Ind. Or.

Nerina N. Holl.

Venilia $1 n d$.

Alimena L. Ind.

Phaerufa L. Ind.

Igatha Ind.

Laura Iamaic.

Lydia Surinz.

Jphicla $L$. Am. $m$.

Liberia Ind.

Amafia Surin.

Aethiopia Afr.

Veronica Guin.

I 3

PAPI- 
Papilio. Gnidia Ind.

Perfea Ind.

Neretea $L$. Ind.

(3) Tipha L.

Procris Chin.

Maja Braf.

Lirymanthis Chin.

Carinenta Surin.

Martha Siam.

F. album $R u$ f.

Harpyja Afr. aeq.

Celtis E Eur.

Melifla $A m$.

Elea L. Iud.

B) Cocila C.

Mefenteria Surin.

Niphe L. Chin.

Thyelia Ind.

Paphia L. Exur. ")

Cynara Or.

Cethofra Eur.

Cytherea L. Ind.

Aglaja L. Est. ')

Aphrodite Am, $m$.

Myrina Ams.S.

Cybele Ant.

Idalia Anz. 6 .

Diana Virg.

Adippe L. Eis.

Lathonia L. Eur.

n) Der Silverftricb. Le Ta. bac d'Efragne. Great Silver. Streaked Fritillary.

o) Der grojse Perlmutzerfalter. Le grand Nucré. The greater Silver fpotted Fritillary.
PAPILIo. Euphrofine $L$.

Eivr.

Selene Germ.

Niobe L. Eur.

Columbina Am.

Bellona $A n$. $b$.

Phalantha Ind. Or.

Thais An. m.

Gripus Ind.

Pofthumus.

Sophorae L. Am.

Xanthus $L$. Af.

Cafliae L. Am.

Berecynthus Surin.

Arcadius Afr.

Honorius.

Oethon Surin.

Polixenes'Am. $b$.

Cupavins Guin.

Conllantius.

Marthefius Surin.

Arcefilaus Siam.

Nero Af.

Polycarmes Suriti.

Acropus L. Ind.

Aucaeus I. Ind.

Obrinus L. Ind.

Lucindus Surin.

Liriope Guia.

Arnuinius.

Morpheus Ant. $b$.

Lyfidice Surin.

Eumenus Cajen.

Dardus Surin.

Arcas Surin.

Canthus Am. $b$. 
Papilio. Eurythris la. Papilio. Sara Guia.

maic.

Hermes Braf.

Irius $N$. Holl.

Mineus L. Chin.

Crantor Ind.

Mergus Afr.

****** Heliconii.

Horta L. Afr.

Quirina Madras.

Calliope L. Ind.

Melite L. Ind\%.

Mopfa L. Ind.

Mneme L. Ind.

Lycalte.

Lyfimnia.

Clara Surin.

Egene An.

Eva Suriu.

Libia $\operatorname{lnd}$.

Vefla Chin.

Hypatia Afr.

Violae Ind.

Terpfichore L. Af.

Serena Ind.

Polymnia L. Am. $m$.

B) Ifabella C.

Hippodamia.

Irene Am. Vera Crux.

Sappho Tamuic.

Vrania $L$. Ind.

Doris L. Surin.

Olympia $A m$.

Crifia Anu. nt.

Hecale Surin.

Ricini L. Am.
Mufca.

Braffolis Surriz.

Thales Sitrin.

Myrti Surin.

Nafica.

Cepha Cajerr. Pfidii $L$. Tnd. Afpafia Tranqb. Aegle Irtd.

Charitonia L. Am. m. Melpomene L. Am.

Phyllis Braf.

Clio L. Ann.

Thalia L. Tud.

Rolalia Surin.

Euryta L. Ind.

Vmbra.

Aedca L. Find.

Thallo L. Chin:

Antiocha L. Ind.

Camoena Afr.

Macaria.

Zetes I. Guin.

Perfiphozie.

Gea Afr. aeq.

Iodutta.

Parrhafia Ind.

Lycia Sierra Leont.

Pyrrha Braf.

Pafiphae Guin.

Marcia Aftr aeq.

Caecilia Afr. a $a q$.

Bonafia Siemra Leors.

Euterpe L. Am.

Sufanna $A f r$.

I 4

Papi- 
PAPIlio. Hyparete $L$ Ind.

Patithoe L. Ind.

B) Egialeus $M$.

Erythrea Surin.

Erato L. Ind.

Helladona.

Iulia $A m$.

Hippona Ind.

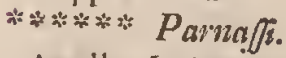

Apollo L. Eur. ${ }^{\text {p) }}$ )

Phoebus Sibir.

Mnemofyne L. Eur. bor.

Crataegi L. Eur. q)

Andromacha $N$. Holl.

Mandane Afr.

Piera L. Ind.

Anacardii L. Am.

Nercis Am. m.

Andromeda Ind.

diaphanus Tamaic.

B) Aflea C.

hyalinus.

obfcuratus.

Idea L. Ind.

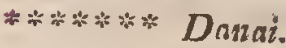

Braflica L. Eur. Af. r)

p) Dor rothe sugenfpiegel. Der Hausmmirzfalter. 'L'Apol. lon. Papillon des Álpes. Alpi. né Butterfly. Gazé.

q) Der Banumbeifsling. Le

r) Der grofse Koblineifsling:

Le Grand P. blane du chorr. Great white Cabbage ButterAly.
PAPILIo. Rapae $L$. Eur. ${ }^{\text {s) }}$

Napi L. Eur. Af. ')

Sinapis L. Eur.

Narica Guin.

Raphani Eus.

Caftalia Ind.

Poppea Sierr. Leon.

Sylvia Sierr. Leon.

Paullina Ind. Or.

Argante Braf.

Monufte $L$. $A m$.

B) Alcyone C.

Amaryllis Ind.

Argia Sierr. Leon.

Fabia S. $N$.

Nephte Chin.

Amathonte Am. $m$.

Phileta Ann.

Libythea Ind.

Calypfo Sierr. Leon.

Daplidice L. Eur. Af.

Cneora Ind. Or.

Neriffa Chin.

Demopbile L. Ind.

Vaneffa Am.

Hecabe L. Ind. Or.

Lybithea S. $N$.

Agave Cajen.

Cardamines L. Eur. ")

\section{PAPI.}

-) Der kleine Koblupeifsling. Le petit P. blane du chou. LefCer white Cabbage $B$.

t) Der Rinhlenumeifsling. Le P. blane veiné du vert. White veined $B$.

u) Der Awroravogel. 
PA P I L I O. Genutia Ind.

Elorea.

Nina Ind. Or.

Eucharis Ind. Or.

Nyfa N. Holl.

Mula Ind. Or.

Chloris Sierr. Lron.

Rhodope Sierr. Leon.

Elathea Am.

Alcmeone Tranqb.

Phryne An.

Licinia Ind.

Aurota Corom.

Zelmire (Libythea et

Cneora non differunt) Tranqb.

Pyranthe I. Ind. Or.

Collirrhoe S. $N$.

Leucippe Ind. Or.

Glaucippe L. Af:

B) Callirrhoe $\mathbf{M}$.

Coronuis Chin.

Eudoxia Sierr. Leon.

Iphigenia Suriss.

Teutonia $N$. Holl.

Myfis $N$. Holl.

Argenthona.

Nigrina N. Holl.

Cneora Ind.

Saba Siery. Leon.

Coronea Surin.

Melania $N$. Holl.

Scylla L. Ind.

Flippantha.

luditi Poulicandor.

Anata Ind. Or.
Papizio. Cypraea Ind.

Danae Ind. Or.

Sefia Ain.

B) Mariannae C.

Rahel Ind.

Meffalina Ind.

Aenippe Chin.

Evippe L. Af.

Charmione Inf. Ioh.

Trite L. Afr. Eur.

Edufa $H i \int p$.

Eupheno Eur.

Belia L. Barb.

Phronima Ann.

Pfamathe Ant.

Palaeno L. Eur.

ß) albida.

Hyale L. Eur. Afr. Ain.

Aurora Sirir.

Nicippe Virg.

Lesbia Patag. litt.

Sennae L. Am.

Larra S. $N$.

Marcellina Surin.

Titonia S: $N$.

Eubule $L$. Carol.

Catilla Tranqb.

Proterpia Iamaic.

Gnoma Ind.

Drya Am.

Philippa.

Rhanni L. Eur. Afr. ")

I 5

Papl.

v) Der Citronenfcbmezterling. Le Citron. Papillon de la Canicule. The Brimftone Buttertly. 
P A P I I O. Philca. L. Ind. Cipris

Maerula Ind

Pomona N. Holl.

Florella Sierr. Leon.

Cleopatra L. Eur. Afr.

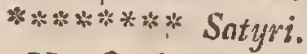

Hypfipyle Eurr.

Tarpejus Ro/f. anft. :

Fortunatus Lapp.

Clytus L. Afr.

Hyperbius $A f r$.

Florimel.

Philocles Ind.

Renatus Surin.

Croefus Survin.

Hyperanthus L. Eur.

Clerimon.

Zachreus.

Ethus Lapp.

Oedippus Ro:j: auftr:

Zangis. Carol.

Phocion.

Solybius.

Perfeus N. Holl.

Martius.

Terminus N. Holl.

Sirius N. Holl.

Cornelius.

Davus Germ.

Pamphilus L. Ear.

Arcanius L. Eur.

Leander Eur. auffr.

Sabaeus Etur.

Hero L. Germ.

Arctons N. Holl:
Papilio. Phryneus Roff: ouftr.

Hypheus Ind. Occ.

Baldus Ind.

Magus.

Dromus Ital.

Eumenus Ind. Occ.

Ephynes Surin.

Tullius Cajern.--

Actaea Rolf.anftr.

Fidia L.' Afr.

Ferula Ital.

Cordula Ital.

Fauna Germ.

Feronia L. Ind. Or.

Maera L. Eur.

C) minor.

Roxelana Af.

Proferpina.

Lycaon.

Alope Ind.

Clueria.

Laches Guia.

Herfe.

Dejanira Germ.

P. la Am.

Bri.sis L. Germ.

Manto Germ.

Autonoc Roffonftr.

Alcyone Rofj. auftr.

Semele L. Eur.

Arethula Germ.

Hermione L. Germ.

Gall.

Ciroe Eur.

Phaedra L. Eur. 
PA P I Y o. Periboea

Surin.

Delila Guirs.

Ligea L. Eur.

Bathfeba Barb.

Medufa Germ.

Epiphron Germ.

Blandina Germ.

Griela Lopp.

Afra Rofla auftr.

Erina Germ.

Arachne Germ.

Pyrrha Germ.

Cafliope Grm.

Arete Grm.

Salome Gibralt.

Galathea L. Eur. ")

Arge Roff: auftr.

Thecla (nec Liria)

Ind.

Philofellae Germ.

Ida Germ.

Melufina Sierr. Leon.

Cadma Anr. $n$.

Ianira L. Eur.

B) Iurtina L. Eir.

Clymene Rol.

Miriam Ind.

Eudora Germ.

Tabitla Ind. Or.

Tifiphone Germ.

Halyma Ind.

Rumina L. Lujat. Eur.

w) Der Marmorargus. Das Bretfpicl. Le Demi - deuil. Common Half-Mourner.
Papiluo. Flegia Cajen.

Allica Siam.

Melicerta Sierr. Leon.

B) Blandina C.

y) Leucothoe C.

Aceris Eur.

Acente L. Ind.

Sulpitia Ind. :

Sibilla L. Eur.

Camilla L. Gernt.

Lucilla Germ.

Coenobita Ind.

Herfilia Am.

Sophia Ind.

Doriclea Ind.

Auge Ind.

Ethelia $A f r$,

Narva Afr.

Mardania Ind.

Cocalia Ind.

Lucina L. Eur.

Cinxia Eus.

ß) Delia M.

Phoebe Eur.

didyma Roff. auftr.

Fafcelis Rolf. auftr.

Athulia Rolf.bor.

Hylla $O r$.

Dikfyuna Ro/f.

B) Athalia uninor $E \int p$.

Cynthia Gernz.

Naturna L. Eur.

Arduinna Ro/f. auff $r$.

Hecate Germ.

Artem is Gersn.

Amathufia Ro/f. 
Papilio, Dia L, Eur.

Dryope Sierr. Leon.

Levana L. Eur.

Prorfa L. Eur.

Pandora.

Pales Germ.

810. Daphue Gorm.

Hesperia.

* Rurales.

Papilio L.

Cupido I. Am.

Guidus Strin.

Agrippa Au.

Helius Afr. aeq.

Ianias Sturin.

Pythagoras Ind.

Amor $7 n d$. Or.

Hefiodus Ind.

Timon Aik, nuer.

Moncus Afr. aeq.

Faunus Sierr. Leon.

Lifias Poulicand.

Hymen Sierr. Leon.

Gabrielis $A m$. $m$.

Pindarus Ind.

Chiton Ind.

Pelion Am. m.

Aunus Am.

Freja Tranqb.

Etolus Ind.

Petus Surins.

Vulcanus Ind. Or.

Ganymedes Ind.

Mars Ani. w.

Celerio Susin.

Ematheon Surin.
Hesperia. Didymaon

Szuriz.

Erofiue C. B. S.

Ifocrates Ind.

Atys Ind.

Sophocles Ind.

Euripedes Ind.

Pholeus Surin.

Anacreon Ind.

Thales Ind.

Endymion Surin.

Venus Surin.

B) Actaeon $\mathrm{M}$.

Echion: Am.

Teleunus Am. m.

Dindus Ind.

Cecrops Ind.

Hemon Surin.

Sphinx Ind. Or.

Cippus S. $N$.

Moliboeus Ind.

Mrecenas Chin.

Tyrteus Ind.

Xenophon Ind.

i.: "rfyas L. Am.

Bathis Surin. .

Cyanus Surin.

Hyacinthus Ind. Oce.

Halefus Am. b.

Achaeus Ind.

Pericles Ind.

Formolus Surin.

Meton Surir.

Thero L. Afr.

Liger Surin.

Harpax Am. 
HESPER 1 A. Valens

Surin.

Perion Surin.

Auguftus $A m$.

Centaurus N. Holl.

Gerontes $A f i$. asq.

Iarbas Siant.

Ceranus Ind.

Pann Ind.

Phorbas Ind.

Betulae L. Eur. ${ }^{\mathrm{x}}$ )

Pruni L. Ear.

Spini Gorm.

Quercus L. Eur. ${ }^{y}$ )

Linceus $R o f . b$.

Cerafi Germi.

Acaciae Roflo auftr.

Symethus $A m$.

Longinus $5, N$.

Boetica L. Eur.

Apidanus Surin.

Aclianus Ind. Occ.

Strephon Iud. Or.

Ericus Ind.

Theophraltus Marocco.

Columella Am. m.

Cleon Braf.

Phocides Afr.

Alcides Sierra Leon.

Philippus Ind.

Atymnus Sinm.

x) Der Nirrenfleck. Le Porte quene à bandes fauver. The brown Hair . Streack.

y) Der kleine Blaufcbiller. Le Porte queue bleu aे une bande blanche, Blew hair.ftreack.
Hesper1a. Eryx Chin.

Aeolus Ind.

Coriolanus Ind.

Plinius Ind.

Acmon Surin.

Democritus Ind. Or.

Amyntas Germ.

Timaeus Surin.

Simathis Surin.

Sedi Grvin.

Phidias Frd.

Herodotus Ind.

Cnegus S. $N$.

Strabo Ind. Or.

Rubi Eur.

Papilio $L$.

Plato Ind.

Rolisinon Ind.

Hippocrates 7nd.

Teocritus Ind.

Lincus Surin.

Parrhafus Ind.

Amyntor Ind.

Narciflus N. Holl.

Apelles N. Holl.

Heraclitus Am.

Plautus Fiad.

Thyfpe L. Afr.

Nais C. B.S.

Meleager Germ. ${ }^{2}$ )

Epulus Surin.

Arion L. Eur.

Erebus 'Germ.

Alcon Germ.

Hespe.

2) Zackichzer blankblauer Faleror. L'Argus blen decoupé. 
Hesper I A. Damon Germ.

Cyllarus Germ. Argiolus L. Eur. Acis Germ.

Alfus Germ.

Cajus Ind. Or. Otis Chin.

Argus L. Eur. ") Artaxerxes Angl. 'Titus Angl. Optilete Ro/f: $b$. Triton Rof.m. Corydon Germ.

B) Tiphys ESp. Adonis Germ.

Dorylas Gern.

Hylus Germ.

Battus Germ. Archiades Saxon. Amphion Germ. Acreon Roff.m. Hebrus Survin. Avius Ind. Acanthus Surin.

Ceneus Survin. Erinus N. Holl.

Icarus Stwin.

Parfimon Sierr. Leon.

Damoetes $N$. Holl.

Ceraunus Am. m.

Catilina Am. m.

Minereus Am. m.

Hylax Ind. Or.

Telephus Am.

a) L'Argus bleu, Blue Ar-
HESPER IA. Enylius

Surin.

Caricae L. Am. Pelops Cajen.

Lamis Cajen.

Cachrys Cajen.

Lagus Surin.

Dimas (nec Maecenas)

Guadel.

Mantus Afr.

Midas Ind.

Bibulus Ind.

Aefopus Ind. Or.

Phaedrus Ind. Or.

Bias Cajens.

Phareus Suriza.

Tacitus Surin.

Coenus Ind.

Procas Surin.

Alphonfus Surin.

Hippothoe L. Eur.

Virgaureae L. Eur. ${ }^{b}$ )

Chryfeis Germ.

Saluftius Ind.

Florus Ind.

Hiere Gern.

Phlacas L. Eur. ')

Arcas C. B.S.

Ballus Hifp.

Eleus S. $N$.

Helle Germ.

Xanthe Germ.

Hespe-

b) Der Feurppapilion. Le Bronzé. L'Argus fariné.

e) Der kienne Fenerfalter. Le Bronzé. Sual tortoife fhell. 
Hesperial Garbas

\section{Germ.}

Therfamon Rofl. auftr.

Lucianus Guadel.

Pentheus Surim.

Vlricus Surin.

Thyra L. Guin.

Zeuxo L. Afr.

Iuba Sierra Leon.

Lara L. Afr.

Cephus Ind.

Domitianus Guadel.

Livius Ind.

Romulus Ind.

Actoris Surim.

Callius Surin.

Ifarchus Am.

Laches $A m$.

Ouranus Savin.

Horaldus Ind. Or.

Talus Surin.

Neleus L. Ind.

Athemon L. $_{\text {. } A m \text {. }}$

Regulus Ind.

Hil bon Ind. Or.

Tarquinius Ind.

Gemellus Cajon.

Ptolomaeus Ind.

Archimedes Ind.

Lucius Ind.

Ovidius Ind.

Suetonius Ind.

Election Cajen.

Sagaris Surin.

Euriteus Surinz.

Phereclus $L . A m$.
Hesperta. Lyfippus $L$. Am.

Amulius Ind.

Geminus Am.

Lucanus Ind.

Perditus Cajeno.

Pyramis Cajerb.

Priaflus L. Ind.

Thucydides Ind.

Virgilius Ind.

Petronius Ind.

Numitor Ind.

Philiafus L. Afr.

Gyas Swin.

Aegon Iamaic.

** Vrbicolue.

Exclamationis Ind.

Phocion S. $N$.

Comma L. Euv.

Propertius Ind.

Tibullus Ind.

Linea Gerur.

Sylvanus Ro/f. $b$.

Thaumas Ams. $b$.

Augias L. Ind.

Bion S. $N$.

Vitellius Am. m.

Colon Ind.

Maro S. $N$.

Panifcus Germ.

B) Silvius E.

Helirius Surin.

Saturnus Cajen.

Origenes Ind.

Noftrodamus Barb.

Phineus Surin.

Helius Surin. 
HeSPERIA. Metis L. C. HesperIA. Thrax $L$.

$$
\text { B. S. }
$$

Diofcorides Tranqb.

Plutarchus lnd.

Epictetus Ind.

Silenus Sierra Leon.

Orion Surin.

Eudoxus Strvin.

Proteus L. Am.

Chemnis Ind.

Tityrus Ams.

Muretus lizd.

Mercatus Ind.

-Epitus Surin.

B) Evadius $C$.

Nicias Cajen.

Mercurius Cajen.

Thyrfis Am.

Alphaeus C. B.S.

Pretus C. B.S.

l'hocas ( $Y$. Amyntas

M.).

Ericus.

Momus Cajen.

Vefpafius ind.

Rhetus Surin.

Clonias Surin.

Acaftus Surin.

Palemon Surin.

Phocion Lannaica.

Brontes Am.

Arinus Surin.

Alexis Inu. Or.

Mithridates Ind.

lupiter Sierr. Leon.

Cafiander.

Polybius Ind.
Ind. Or.

B) Sebaldus C.

y) Ramafis $C$.

Cremius S. $N$.

Ennius Ind.

Scipio Ind.

Marcus Cajen.

Flefus Afr. aeq.

Cicero Inid. Or.

Atticus Ind.

Clericus Am, mer.

Nero S. $N$.

Mathias S. $N$.

Lucas Ans. $m$.

Iuvenalis $A m$.

Nepos Ind.

Nerva Ind.

Cacfar Ind.

Tertullianus Ind.

Orcus Ind.

Dan Tranqb.

Nothus Am.

Silvius C. B. $S$.

Salus Surin.

lacchus $N$. Holl.

Gnetus Ind.

Alfarius lnd.

Anaphus Surin.

Sebaldus Am.

Evadrus C. B. S.

Bixae L. Am.

Polycletus L. Ind.

Aracinthus Germ. Ro:f.')

HESPE. roir. 
HESPERIA. Bufiris Ind. Pififtratus $A m$. Henes Surin.

B) Phoreus C.? Eumelus Surin. Philemon Ain. Thrafibulus Ind. Celfus Ird. Zeleucus Ind. Maenas $A m$. Phidias L. Af:

B). Acaftus $C$. Amiatus $A$ m. Aefculapius Am. $b$. Lycagus Surin.

Catullus Ind.

Iovianus Ind. Salvianus Ind. Syrichtus Am. m: P. Oileus $L$. Maimon Cajen. Talaus $L$. Ind. Mimas Surin. Gentius Suriz. Pelopidas Ind. Galenus Ind. Malvae I. Eur. ${ }^{\text {C) }}$ Alceac Ro/f. m. Sidae Roff. auftr. Fritillum Germ.

ß) P. Malvae, major ESp.

Galba Tranqb. Maevius Ind. Or.

e) Le Plcin chant. Brown March Fritillary.
HES P ER I A. Lavaterae

Germ.

Menalcas Ind.

Menippus Surin:

Clito Cajen.

Virbius Surin.

Curtius Surin.

Phocion Afr. aeq.

Tages L. Eur.

Nifo L. Cajen.

Spio L. Ind. 36r. pygmaea Ind. SpHivx. (Dämmerungsfalter. Le Splinzx.) ocellata L. Eur. Amo ${ }^{5}$ )

Neffus $A m$. $b$.

Quercus Germ. modefta Trantqb. lugubris $L$. Ann. $m$. lufca Am. m. Inf. afilifornis Ind. feneftrina Germ. Populi L. Eur. Tiliae L. Eur. ${ }^{g}$ ) Rhadamiftus Sierr. Leon. Oenotherae Eur. ${ }^{\text {h) }}$ ) Nerii. L. Eur. Am. ${ }^{\text {) }}$ ) Apulus Surin. Penzeus $A m . m o$ Pylas Survin. SpHiNx.

f) Le Demipaoti.

g) Le Sph. du Tilleul.

h) Der Nachtkerzenfchäär. mer. k

i) Der Olcnnderychivarowses 
SPHINx." obfcura Am. mer.

Cacus Surith dentata Ind. Alope Am. Ello L. Surin. Iatrophae Ann. B). Modor C.? Medea Afro aeq. Pholus Ind. Occ. carolina $L$. Am. Atrigilis St. Crucis. Atropos L. Eur. Or. Ain. ")

Lachefis S. $N$.

Erotus Ind. equeftris $I n d$. Or: Phorbas Ind.

Ficus L. Ain. Inf.

Tetrio Am. m. ruftica $A m$.

pagana $I n d$. $O r$.

Pinaltri L. Eur. plebeja $A m$.

Euphorbiae L. Eur. ')

Gallii Germ. ${ }^{\mathrm{m}}$ )

lineata Ewr. Am.

Vefpertilio Eur.auftr.

ß) Minus $M$.

y) Vampyrus $M$.

k) DerTodzenkopf. Steshap. felfchuärmer. Le Sphiinx à tête de mort.

i) Der Wolfsmilchychwärmer. Le Sphinx de Tithymale.

ii) Der Waldftrobjchioür. mer. Le Sphinx du caille-lait.
Sphinx, Vitis L.Am. fatellitia L. Iamaic. Celerio L. Eur. auftr. Oldenlandiae. Lycetus Ind. Or. Boerhaviae Ind. funebris Guin. didyma $A m$. Hefpera Ind. Or. Parce Braf. Elpenor L. Eur. Porcellus L. Eur. Hylaeus $A m . b$. Convolvuli L. Eur." ) Liguftri $L$. Eur. ${ }^{\circ}$ ) Abadonna S. $N$. cingulata Ann. m. Caicus Survin. Crantor Ind. Or. Alecto L. Ind. Clotho C. B.S. Gnoma Ind. Anubus Surin. Neclus Amer. Batus Ind. Clio Guinto Labrufcae $L$. Am. Caricae L. An. velox Ind. Or. terfa Am. m. 72. Thyelia L. Ind. SESIA.

Sphinux

n) Le Sphinx à cornes dı bocuf.

o) Der Rainweidenfcbuärpmer. Le Sphinx du Troënne. 
Sphinx $L$.

Sesin. Tantalus $L$, Surin.

Etrr.

B) Ixion $M$.

Melas Survin.

Hylas L. Chimz.

Fadus Surin.

flellatarum $L$ Eur. ${ }^{p}$ )

Pandora Inci. Or.

brunnes Survin.

8 maculata $1 n d$.

Marica Surin.

Thysbe $A m$.

fuciformis $L$. Eus?.

bombyliformis Eur.

apiform is $L$. Eur.

chaleiformis Trnqqb.

fphegiformis Germ.

afliliformis Eur.

crabroniformis Ital.

haemorrloidalis Ind.

culiciformis L. Eur.

tenthrediniformis Eur.

tipuliformis $I_{\text {. . Eur. }}$

ichneumoniformis Germ.

23. vefpiformis L. Eur.

ZYGAENA.

\section{Sphinx L.}

Filipendulae Eur. 9)

B) Peucedani Efp.

Scabiofae Germ.

B) Pilofellae E.

Loti Eur.

Lavandulae Gall.

p) Le Moro Sphinx.

q) Der Erdeichelfchwärmer. Le Sphinx Belier.
ZYG AENA. Sedi RolJ. mo

Quercus Germ. ')

Sph. Phegra L.

collaris Ind. Or.

Coronillae Evr. s')

Sph. Ephialtes L.

B) Falcat W.

Aeacus Germ.

Agrius Stwin.

ainulats $N$. Holl.

Onbrychis Germ.

B) cingulata.

caffra.

guineesfis.

Palfalis Ina. Or.

Cerhera $L$ ititiop:

Thetia L. inaliaic.

Phlegunon Stwin.

Thelebus Chin.

Sperbius Chin.

feneftrata Chin.

Caffaudra L. Am.

Hyparchus Sierr. Leon.

Argymis Rraf.

Myrtea Brof.

Andromacha Am.

B) Caunus C.?

flavicornis Cajen.

Bromus Surin.

Eryx Am. $m$.

Egeon Chin.

$\Lambda$ chemon Iamaic.

$\mathrm{K} 2$

ZYGAE.

r) Der Kabneichenfibwärmer.

Le Sphinx de Piffenlit.

s) Der Sichelklecjcismärmer.

Le Sphiax de la Luzerne. 
ZYG A ENA, nigricornis Cajen.

Melifia $A m$.

Polymena L.Chin.

Lethe Afr.

Lichas Arab.

faufa L. Eur. anftr.

infauta L. Eur. auftr.

Eriphia Surin.

flabellicornis Am. m.

Butus $A m, m$.

Pugione L. Am.

Eurncilia Am.

pectinicurnis L. Am.

Gyon Cajen.

Ergotis Mader.

Glaucopis Carol.

Eryinis Surin.

Pylotis Surin.

Sypilus $A m$.

Megaera Guian.

Scyton Amboin.

Auge L. Am.

8 maculata $A m$.

capiftrata $A m$.

Parthenii Am. $m$.

Colombina Am. $m$.

caudata $A m$. $m$.

diptera $1 n d . O r$.

halterata Braf:

Maja Anz.

tibialis Afr.

Jadis Surin.

Eunolphus An. $n$.

Panthalon Am. m.

Capys Suriz.

Pholus Afr.
Z Y G A E N A. Statices $L_{\text {. }}$ $\left.E u{ }^{t}{ }^{t}\right)$

Pruni Germ.

Acharon N. Holl.

Styx Afr.

72. Thoas Ind.

BомBYX.

Phalaena L.

* alis patulis.

Atlas L. Am.

Hefperus L. An.

Aurotus Ant.

Cecropia L. Am. b.

Irius Ind.

Saturnus Ind.

Paphia L. Afiae.

Polyphemus $A m . b$.

Dione Guin.

Cytherea C. B.S.

Mylitta Ind.Or.

Promethea Ann.

Erythrinae $A m$. $m$.

B) Caffandra $\mathrm{C}$.

Confpicillator Afia.

Janus Surin.

Certhia

Megaera Am. $b$.

Hippodamia Surin.

nictitans $A f r$.

Semiramis $A m$. $m$.

Boreas Am.

Luna L. Am. $b$.

Epinethea Guin.

ВомвуX.

t) Der Seenelkenfiniminer. Der Taubenbals. Der Thykis, Le Turqquoife. 
Bомвух. Argus Sierra Bомвух. Auftralafiae.

\section{Lean.}

Feneftra L. Ind.

Penelope Ind.

Tyrrhea C. B.S. perfpicua $A$. lnd. Armida Cajen. militaris L. Afrat. Caftalia N. Holl. Pavonia L. Eur.

a) minor.

B) media.

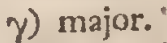
Achelous $A m$. argulata Surin. Liberia Sarin. Tau L. Eur." ) Io Am. $\Lambda$ bas Surin.

3) Abafia C. Salmonea Surin. Proferpina $A m$. * alis reverfis. populifulia Germs. quercifolia L. Eur. v) ilicifolia $L$. Eur. Promula Iavae. brunnea S. $N$. Caffandra. capenfis. orientalis. Aluco $C . B S$.

i1) Dor Nagelfleck. Der TVogel. Der Rotbbuchenfpinner. La Hachette du Soiffonnois. feches.
4 cincta Ind. Or.

Fagi L. Eur.

Trifolii Germ. ")

Quercus L. Eur.

Stigma Am. $n$.

lufca Coron.

Pruni L. Eur.

Amphimone, Terra del Furgo.

potatoria L. Eur.

ocularia Am.

Hibifci Ind. Or.

Cynera Surin.

Pini L. Errr.

Dumeti L. Eur. ${ }^{\mathrm{x}}$ )

verficolora L. Eur. ${ }^{y}$ )

Rubi L. Eur.

lunigera Eur. vinula $L$. Eur. ${ }^{\text {z) }}$ )

B) Erminea E. lanefris L. Eur. rubicunda Virg. Populi L. Eur. Catax L. Eur. Everia Germ. proceflionea L. Errr. pithyocampa Germ. K 3

BOMBYX.

w) Der kleine Qnittenungel.

x) Der Habicbeskrautpinner. Dir Lömenzalonjpinner. Der Gelbpunkt.

y) Der Buntfizgel. Der Hagenbubenfpinner. Le Verfi. colore.

z) La queuc fourchue. 
Bomiry. varia (rurea M.) Bombyx. Helops An. Germ.

Mori L. Chin. ") tricolora Cajen. ferruginea Ital. neuftria Germ. ") caftrenfis L. Eurr. americana. franconica. Taraxaci Germ. ") lobolina S. $N$. Ilicis Gall. Mali Eur.

ß) Avellanae M. Hieracii L. Eur. apifurmis Ital. ****alis deffexis. Lagopus Chin. Impcrialis It d. craflicornis Ind. Hyphino Asub. Cyane Anboin. regalis Ant. b. difpar L. Eur, d) Lantanae $A n$. m. Amafis Surin. I pudibunda L. Eurr. fafcelina L. Eur. bucephala L. Eur.

a) Ver à Snje. Silkworm. b) Der Weisbuchenfinturer. Die Bisurvingelnotte. Dir Str:inwwingervogel. La livrée des arbres

c. Der Butzerblismenfizinner.

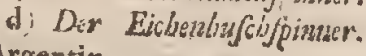
L'Argentin. oleagina Germ. e) coeruleoceph. $L$. Eur. argentina Germ. ${ }^{\mathrm{f}}$ ) decora $L$. C. B.S. Celfia $L$. Suec. Dione Ant. b. Ziczac $L$. Eur. tritophus Germ. elegans $C$. B.S. Camelus $1 n d$. Dichaea Eur. Gnoma Germ. Dromedarius L. Eur. Coryli L. Eur. nuda $I n d$.

Morio Eur. Rubea Germ. Alphaea $N$. Holl. monacha L. Eusr. flava Ind. Intea $N$. Holl. ephippiger $A m$. Inf. curtula $L$. Eurr. reclufa Germ. anachoreta Gernn. anaftomofis $L$. Eur. Cippus Surrin. reflexa $A m$. m. Inrf. palpina L. Eur. trepida Germ.

BoMryx.

e) Der Sebleenfpirner. Le Pácifique.

f) Der Rosenfoinner. Die Stammmootte. Der Grofskopf. Le Zigzag. 
Вомвух. querna Germ. capuzina L. Eur. carmelina L. Eur. aulica $L$. Eur. hepatica S. $N$. helvola Germ. undata Germs. Acria Carol. lubricipeda $L$. Eus. mendica L. Germ. Menthafri Germ. virginica $\mathrm{S} . N$ : advena $\mathrm{Hi} / \mathrm{S}$. transverfa S. $N$. rutila Sians. luctifera Germ. ') leporina $L$. Eur?. lota $L$. Eur. ftrigofa $L a p p$. laeta Suec. communimacula Eur. auftr. compreffa Germ. Milliauferi Germo fpreta Germ. Lincus Surin. Vlmi Germs.

Begga Surin. obfoleta $A m$. wr. lepida Tranqueb. equeltris $I n d$. grata Georg. Amer. V. nigrum Gernn. chryforthoea $L$. Eur.

c) Dir Trauerfalter. Das Kaifervögclein. Dor Spitzopege. richsspiziner.
Bombyx. auriflua Germ:

bicolora Germ.

Salicis L. Eur.

Caffinia Germ. centrolinea Germ.

Crataegi L. Eur.

Eridanus Surin. tibialis N. Holl. nitidula Corom. innocua $A m . m$. illibata $A m$. repanda Am. m. exigua Am. m. plumigera Germ. obfoleta N. Holl. Corones N. Holl. feltiva Anu. Dryas Surin. ramola Ital. ruffula $L$. Eur.

B) Sannio L. Iacobaeae $L$. Eur. grammica $L$. Eur. penuatula Ind. Or. lineola $\mathrm{Ind}$. Or. purpurea $L$. Eur. Plantagingis L. Eur. ") vitcata Am. lugubris En!r. ${ }^{\text {) }}$ ) $\mathrm{K}^{\prime} 4$ Bombyx.

h) Der Wegerichsfpinner. Die Jpanifcbe Falmes: Die befcbleierte Bävenpbalene. Le ecaille brune.

i) Die Windcnmotte. Der Scbsefflgelle Naclutfalter. L'Ar. lequiuette jaune. 
Ph. Sulphuralis $L$.

B м в х . heliophila

$$
\text { Lapp. }
$$

vidua Eur.

$P h$. parthenias $L$.

matronula L. Eur.

villica $L$. Eur. " )

Hebe L. Eur. ')

Tarquinius Suritu.

Tarquinia Surin.

Caja L. Eur. ' ${ }^{\text {) }}$

Flavia S. $N$.

pudica Gall. auftr.

cafta Germ.

maculofa Gall.

Virgo L. Am .

Menete Am.

deflorata $A$ in.

hyalina Ital.

**** alis incumbenti-

\section{bus.}

Crotalariae $I n d$.

Ricini Ind.

fanguinolenta Ind. Or.

puella Guin.

ferva Ind. Or.

Hera L. Eur. ")

Dominula L. Eur.

k) DieMayerin. Der folbrow. ze Bär. L'Ecaille marbrée.

1) Die Wittue. Der Garbenfpnner. L'Ecaille couleur dẹ Rofe. marte.

m) Der Bär. L'Ecaille

nj Der Beinwelifpinner. Die Hausfrau. Die Spanifche Fubne. Le thalenc chinée.
Bombyx. credula An. lectrix L. Chin. furcula L. Ear. ?) colon Germ. teftacea S. $N$. Populeti Eur. antiqua $L$. Eur. gonoltigma $L$. Eur. paradoxa Roff: Zona Germ.

2 frigata S. $N$. Vmber Surin. Hiftrio Am. Inf. Pylotis N. Holl. bella L. Am. $b$. pulchella L. Or. ornatrix $L$. Am. grifea Cajen. priverna Survin. Francilca Coroms. Iefuita Ind. angulata $\mathrm{Ind}$. Or. viciella Germ. veflita Germ. mufcella Germ. bombella Germ. pectinella Germ. munda L. Eur. derala Ind. Or. annulata Germ. Graminis L. Eur. popularis Rofl. Germ. fulminea Germ.

POMBYX.

o) Der Pappeluneidenfpinner. Phalene petite queuo double. 
Bonвyx. Gloriofae Ind. Hepialve. Afellus Germ.

\section{Or.}

Crini Ind. Or. rofea Germ. P) rubricollis L. Germ. ${ }^{q}$ ) fuliginnfa L.Germ. ")

2 notata Suec.

Cribrum L. Eur. b. obfcura Germ.

Ph. ancilla L.

258. punctata Ital.

Cossus.

Phalaena L.

Ligniperda L. Eur. ") unguiculatus Ital. Terebra Germ. Aefculi L. Eur. fcalaris Chin.

6. pyrinus Am. b.

Hepialys.

Phalaena $L$.

Humuli L: Eur. Iodutta Germ. lupulinus $L$. Eur. b. hectus $L$. Eur. obliquus Gall. carnus Germ.

Crux Hafin. Teftudo Germ.

p) Der rofenrozbe Spinzer. La Rofette.

q) Der Purpurhals. Der Rotbibals. La Veuve. pferfpinner.

r) Dir Rojsfiugel. Der Am-

s) Le Coffits.
I 0 . Bufo Germ.

Noctva.

Phalaena $L$.

Phalenes à tête do hi-

bou.

* alis patulis.

Zenobia' Stwint.

Patroclus L. Ind.

Strix L. Am. m.

Bubo Chin.

$P h$. neerops $L$.

Iphianafle Surin.

Aluco Chin.

Caprimulgus Chin. odora L. Surin.

B) Agarifta C. Mycerina Surin. hieroglyphica, Ind. Or.

Vlula Ind. mutabilis Ind. Or.

Itynx Corom.

Noctilio Ind. Or.

Chalcis Am. m.

Spiralis Chin.

Nyctea S. $N$.

crepufcularis L. Am.

troglodyta Guin.

Vampyrus Ind. Or.

fqualida Coroms.

pandrofa.

umminea Iava. Vefpertilio Tranqb. achatina Ind.Or. illibata Ind.

K 5

Noctva. 
** thorace laevi, alis pla- Noctra. cribraria Ind. nis incumbentibus.

Or.

Noctva. Diofcoreae Ind. Or.

\section{Ph. fullonica $L$.}

B) Pomona $C$.

materna $L$. Ind.

Salaminia Ind. Or.

ancilla Ind. Or.

microrhoea $N$. Holl.

ferva $N$, Holl.

Tirrhaea C. B.S.

Paphos Sianz.

Chione Ind. Or.

Aftrea $N$. Holl.

Eugenia Ind.

Narciffus Chin:

Bajularia Amboin:

lacta Gall.

polita Germ.

tetra Germ.

livida Gernn.

irrorata 1 lud. Or:

Quercus Eur.

notacula Germ.

4 punchata Germ:

pallens L. Eur.

domeftica Germs.

nervola Germ.

Sidae Guin.

Vaccinii $L$. Suec:

depceffa Am. $b$.

complana $L$. Eur:

quadra L. Eur.

B) deplana L.

bicoloca Sierr. Leon.
Delia Inf. Amft.

Capreae Hafir. convoluta Malab. litt. narbonea $L$. Ezrr, auftr. Boleti Germ.

*** thorace laevi, alis de: fiexis.

Ficus Ind.

Caricae Ind. Or. coronata Chin.

Manlia Afiat.

Hircus Guin.

Geryon Afr. aeq.

leonina Ind.

Dama N. Holl.

marginella Am.

Arundinis Germ.

virefcens.

Batis L. Eur.

Silene Germ.

nitida Germ.

rubiginea Germ:

rufina $L$. Eur.

martia Germ.

Atrigofa Germ.

Fulvia L. Ind.

fcutola Germ.

glyphica $L$. Eurr:

dipfacea L. Eur.

Ononis Germ.

Mi L. Ezwr.

triquetra Germ.

Punctum Tnd. Or:

pulcra Ind. Or.

Noctva. 
Noc т v A. avidá Ind. Or. Nóctva. electrica Germ..

Roboris Germ.

mixta Germ.

Crucis $A m m$. inquinata Ind. Or. albicollis Eur. italica.

barbata Am. m: palliata Gornn.

Hypatia Ind.Or. Triangulum Tranqb. palmata $A m$. $n$. arcuata Tranqb. vulpina $I n d$. Or. notata Ind. Or. deftituta Chin. tigrina Ind. Or. B) Melicerta C. lyncea $1 n d$. Or. geometrica Ind. Or. Mezenteria Ind. Or. ftolida Ind.Or. niver $L$. Norte. Ioviana Ind. Or. fupola Ind. Or. inclyta Braf. miniofa Germ. urfina Am. mer. canina Ann. Ins. trapezina L. Ezur. Cerafi Angl. Germ: inftabilis Germ. humilis Germ. centralis Germ. Lychnidis Germ. fixa Gibralt. monilis Angt. undata Ind. Or.

vittata Ind. Or.

B) fcrobiculata M. annulata Tranqq. munda Germ. blanda Germ. anbigua $G$ erm. gracilis Germ. gemina S. $N$. repanda Am. m. verficolor Am. Inis. Corchori Am. m. difcolor Trangb. fordida Tranqb. fafciata S. $N$. frugalis $I n d$. flava $I n d . O r$. ftigmatizans Ind. dorfalis Tranqb. cinerea Suec. rejecta Ind. Or. fortificata Germ: elata Ind. Or. lanceolata Germ. ***** thorace criftato, alis incumbentibus. fponfa L. Eur. ') nupta $L$. Eur. pacta L. Eur. promiffa Germ. Spectruin Eur. auftr: Fraxini L. Eur. dotata Ind. Or. formofa Sierr.Leon. Noctva:

t) Die Rotbeicheneule. 
Noetva, pronuba L. Eur. Noctva. rectangula dimidiata Guin. cytherea Suec. orbona Gerrn. Solani Eur. lisogrifea Germ: hymenaea Germ. rapta Tranqb. Epione Am. b. paranympha L.Germ. finbria $L$. Germ." ) Ianthina Gorm. meretrix Germ. alienata Sierr. Leon. Paphia An. m. Augur Germ. fegetis Eur. auftr. ambufta Germ. fubeufa Germ. retufa $L$ Germ. mercatoria Ind. Or. partita Tizd. maura L. Germ. lunaris Germo. luioria $L$.

Craccae Germ. libatrix L. Eur. ") plecta $L$. Eur.

C. nigrum L. Germ. Signum Germ. Nun atrum Germ. cincta Germs.

Die Saumpbaläne.

i) Die Scbliffelblumeneule.

v) Der Dotzerzaeiden fpinner. La Decoupure.

Germ. polymita $L$. Eur. Braflicae $L$. Eur. flammatra Germ. Cefpitis Germ. Chenopodii Germ. grifea Germ. contigua Germ. pupilla Germ. dentina Germ. '2 maculofa Germ. exclamationis L. Eur. diftincta $D a n$. fubter runea $A m$. fuffufa Germ. figuifora Germ. valligera Germ. caecimacula Germ. I. albun L. Eur. pagana Ind. viminalis Germ.

* * *** thor. crift. alis deflexis. fulvago L. Eur. croceago Germ. albago Tranqb. aurago Germ. fulphurago Germ. cerago Germ. rutilago Eur. citrago L. Eur. luteago Roff. mer. ferrago Germ. flavago Germ. gilvago Germ.

Noctva. 
NocT Y A. chryfitis $L$. Eur. ")

concha Germi. orichalcea Ind. lamina Germ. bractea Eur. anslr. Feftucae L. Eur. ${ }^{x}$ ) circumllexa L. Germ. Artemifize Germ. Gamina L. Eur. mouta Germ. aemula Germ. divergens Germ. argentina $R o / f$. interrogation. L. Eutr. fignata lnd. quaeflionis $I n d$. Peponis $7 n d$. Or. Iota L. Euw. verruca $A m, m$. lunata C.B.S. emarginata Tranqb. pauperata Chins. meticulofa L. Eur. ciliata Cajen. confona Germ. illuftris Germ. lacera Chin. oo L. Eur. fainmea Germ. gothica L. Eur. b. derafa Eur. doré.

w) Die Goldeule. Le vert

x) Der Sumpffcbroingel. Der Goldglanz. Phaléne dorée a taches argentées.
Noctva. renata Dan.

Serici Iap.

or Germ.

diluta Germ.

ruficollis Germ.

fatellitia $L$. Eur.

diffinis $L$. Eur.

affinis $L$. Eur.

triptera L. Eur.

marginata Eur.

Abfinthii L. Eur.

Abrotani Germ.

occulta L. Suec.

Patibulum Ind. Or.

albipes $I n d$. Or.

Alni L. Eur.

Delphinii L. Eur. ${ }^{7}$ )

purputina Germ.

Pteridis Germ.

picta Germ.

geographica Germ.

Hyperici Germ.

perfpicillaris L. Eur.

Linariae Germ.

PiG L. Eur.

mendica Germs.

Oxyacanthae L. Eur.

brunnea Germ.

culta Germ.

compta Germ.

prafina Gernu.

Atriplicis L. Eur.

Dolichos An. m.

finuata Ind.

Noctva.

y) Die Ritter/porncule. 'L' incarnat. 
Noc t v A. hiftrionica

$$
\text { Ind. }
$$

Goflypii $A m . m$.

Brafficaria Am. m.

fetofa $A m$. m.

praecox L. Germ. ${ }^{2}$ )

pyranidea L. Eur.

lucipeda Germ.

pyrophila Germ.

typica L. Germ.

lucipara $L$. Eur.

oleracea $L$. Eur.

Xanthographa L. Eur.

leucomelas Germ.

convergens Germ.

rivularis Eur.

ß) capfinicola W.

fercna Germ.

Pinaltri L. Eur.

Liguftri Germ.

ftrigilis $L$. Suec:

runica Eur.

aprilina L. Eur.

virens $L$. Germ.

feladonia Suec.

ludifica $L$. Eur.

Panthea S. $N$.

perla Germ.

fulminans Germ.

Lichenis Germ.

Algae Germ.

tridens Germ.

Pfi L. Eur.

auricoma Germ:

Lamda Germ.
Noctva: Comma $L$. Eur.

Linea $A m$. $m$.

luf:a Germ.

Chi L. Eur.

Aceris L. Eur.

Arnicae Sule.

Euphorbiae Germ. megacephala Germ.

litura L. Eur. depuncta Surc. piftacina Germ.

Baja.

rubricofa Gern. erythroceph. Gern. grifefcens $G$ erm. polygona Germ. barbara. Perficariae L. Eur:

Tragopogonis L. Germ. liepatica L. Suec. Acetofellae Germ.

turca L. Suec. Gerem: imbecilla Gern. conigera Germ. ${ }^{\text {a) }}$ albipuncta Germ. polyodon L. Eur. flavocindla Germ. eremita $G_{c r m \text {. }}$ nigricans $L$, Germs. Suec. perflua Germ. fumofa Gesm. aurita Hifp. oculea Suec. myopa Dan.

2) L'Etoilóe.

Noctva, 


\section{G $\operatorname{lo} \rho \int a t a$.}

Noctra. flavicornis $L$. Hybla ea. Saga Coroms:

Enr.

calvaria Germ.

triplacia L. Eur.

Afclepiades Gexm.

mixta Ital.

trifiis Suec.

Rumicis L. Eur.

hecta Germ.

ridens Germ.

exoleta L. Eur.

deperdita Germu.

Verbafci L. Eur.

Afteris Germ.

Chamomillae Germ:

Tanaceti Germ.

Lactucae Germ.

umbratica L. Germ.

Suec.

lactea $R o f$. auftr.

putris L. Eur.

lithoxylea Germ.

petrificata Germ.

rhizolita Germ.

conformis Germ.

advena Germ.

bafilinea Germ.

radicea Germ.

rurea Angl.

Myrtilli L. Germ. Suec.

Vitis idaeae $S N$.

383. Arbuti Angl.

HyBLAEA.

Lagopus $7 n d$. Or.

roltrata $1 n d$. Or.

deflorata $I n d$. Or.

feabra S. $N$,
Sagitta Ind.

7. gemina Ind. Or.

PHALAENA.

* antennis pectinatis.

lactearia L. Eur.

nivearia Angl.

veruaria $L$. Eurr.

bupleuraria Germ.

thymiaria $L$. Germ.

triftiaria Ital.

inmacularia Tranqb.

putatoria $I$. Eur.

ftriataria L. Eur.

margaritaria L. Eur.

margaritata $L$.

punctaria L. Eur.

Dioraria Surin.

amataria $I$. Eur.

pennaria L. Germ.

bidentaria Eur.

bidentata $L$.

falcacaria L. Eur:

cultraria Germ.

harparia S. $N$.

frigaria Ind. Or.

fambucaria L. Etur. ')

lacertinaria $L$. Eur.

erofaria (nec craffaria)

Germ.

enuarginaria Germ.

equeftraria Germ.

alniaria Lu, Germ. Satec.

Phalae-

b) Der Holunderfpanner. La Soufrée à queute. 
Phalaena: fyringaria $L$. Phal aena. angularia

Eur. ")

lunaria Germ.

dentaria Angl.

roboraria Germ.

coníortaria Germ.

undaria Ind.

hortaria Ital.

dolabraria $L$. Germ. Angl.

illyraria Surin.

moniaria Ind.

fervidaria $A f r$. aeq. rondelaria Ind. Or. vibicaria L. Eur. flavaria $1 n d$. Or. papilionaria L. Eur. macularia Ind.

Papilio Helcita $L$. cateuaria Ind. flabellaria Afr. aeq. prunaria L. Eur. furcaria Germ. piniaria L. Eur. limbaria Angl. fulphuraria Barb. albidaria Afr. aeq. confperfaria Germ. roraria Germ. melanaria L. Eur. lutcaria Ital. teflaria Barb. 2dfperfaria Germ.

c) Das Goldkliimgen. La
Germ.

atomaria L. Eur.

B) glarearia W.? profapiaria L. Eur. bipunctaria Germ. Lichenaria Germ. cineraria Girm. pufaria L. Eur. ocularia Ind. lufcaria Ind. Or. annularia Germ. capreolaria Germ. . proximaria Germ. murinaria Gersu. defoliaria L. Eur. hirfutaria Eur. pedaria Germ. hifpidaria Germ. hirtaria L. Eus. horridaria Germ. vefpertaria $L$. Germ. Angl.

Wawaria L. Eur. pictaria Germ. nivearia Germ. cretaria Ital. pluviaria Germ. prafinaria Germ. fmaragdaria Germ. ditaria Germ. viridaria Angl. feftivaria. pagaria Chin. 3 punctaria L. Afat. corvaria $A m$. $m$.

Phalae. 
PHA I. AENA. carbonaria PHALAENA. aureolaria

- Germ.

Iatropharia $L$. $A m$. Juctinaria $/ n d . O r$. catilinaria Suriu.

$$
\text { B) Tirefias C.? }
$$

cenaria Chin. coftaria.

Jateraria Ind. feneftraria Chinz. venaria $l i t d$. moeniaria Germ. obliquaria Siam. pulveraria L. Eur. fafciaria L. Eur。

B) prafin. W. diverlaria Augl. marginaria Germ. betularia I. Eur. d) nitidaria Euv. auft. prodromaria Germ. facraria $L$. Afr. elinguaria L. Eur. grifaria Eur. crenaria Germ. plumbaria $A n g l$. lignaria Germ. fulvaria Ital. rotaria S. $N$. grataria S. $N$. purpuraria L. Eur. arteforia Germ.

d) D:r Birkentpanuter. Phalene blanche tachetée de noir.

\section{Germ.}

prararia Barb. gilvaria Germ. herbacia $A m$. $m$. brumnearia Ital. fuliginaria L. Suec. minutaria Ital. pygmaria Germ. * antenuis fetaceis. politata Surin. caudata lava. lretata lnd. Or. falciata Ind. angulata $A f r$. aeq. cynata Surin. arenata Tranqb. regulata $B_{r} a j$. falcata Angl. flexula Germi. mantata Surin. liturata Ind. glaucata Barb. netrata Am. m. phylirata Surin. demandata Germ. explanata Chin. Rhamnata Germ. dubitata Grmm. biftrigata (nec dimidiata) Angl. cinerata Germ. irrorata $I n d$. Or. flavata Ind. Or. togata S. $N$. cariuata $I n d$. Or. 1. Phatar. 
Phalana. viridata Lo. Pháana. equeftrata

Etsr. ")

furvata Germ.

lividata Germ.

obfcurata Suec.

Arigata Angl.

notata $L$. Eur.

pulchellata Ind. Or.

nitata Germ.

areata Surin.

fponfata Sierra Leon.

lufcata Cajen.

decorata lamaic.

porata $L$. Eur.

emarginata L. Eur.

bicolorata Surin.

groffulariata L. Eur. f)

gravidata Tranqb.

rofata $A m b o i n$.

undulata $L$. Eur.

hefperata Ind.

flaveolata L. Iird.

populata L. Eur.

pyraliata Germ.

comitata L. Eur.

ulmata $L$. Eur.

colorata Seland.

dealbata L. Germ.

qurata Surin.

prunata L. Eur.

crataegata L. Eur.

aurantiata Germ.

e) Der Brombcerfponner. Arpenteufe de la ronce.

f) Der Stachelbeerfpinner. Der Tiegcr. La mouchetée.
Germ.

dimidiata Cajen.

lucidata Surin.

pudorata Barb.

marginata L. Eur.

miata L. Eur.

rubidata Grrm.

coerulata Germ.

rufata Eur.

hirtata Ital.

fpartiata Gersu.

berberata Germ.

albicillata $L$. Eur.

illibata Gerns.

haीata L. Eur.

triftata L. Eur.

clathrata L. Eur.

diverfata Germ.

averfata L. Eur.

clavellata Ital.

fyerata Surin.

afiuata Gern.

chaerophyllata $L$.

Eivr.

procellata Germ.

flucluata L. Eur.

obliquata Gall.

reticulata Germ.

fordidata Germ.

bilineata L. Eur.

bimaculata Germ.

rubiginata Geriis.

centaureata Germ.

aduftata Germ.

volutata Gerir. 
PHALAENA. fimbriata

Germ.

4 fafciata L. Germ. propugnata Germ.

fulvata Stuec. ocellata L. Eur. Jynceata Angl. rufticata Germ. rivulata Germ. mellinata Germ. atomata Germ. janata $L$. Ind. brumata L. Eur. linariata Germ. exfpectata Afine. ventitata Germ. chenopodiata L. Eur. centuminotala Germ. marmorata Itnl. Atrigulata Seland. lineolata ltal. myopata Ital. hexapterata Germ. dnplicata Eur. Ph. plagiata $L$. lunulata Germ. alchemillata $L_{\text {. }}$ Eur. flammata Ital. pfittacata Germ. aerata S. $N$. lućtuata Germ. rectangulata L. Eur. fuccenturiata $I$. Eur. ferrugata $L$. Eur. pofticata Seland. maculata L. Eur.
PH A I EN A. moniliata Germ. enphorbiata Germ. laevigata Germ. punctata Angl. venufata Germ. murinata $A n g l$. inuata Gall. mediata Germ. fociata Eur. fagittata Germ. omicata Ital. luteata Girm. oblipata Barb. centrata $G$ irmb. cinerata Germ. fulcuta Seland. coarctata Germ. ornata Germ. purpurata L. Eur. rubricata Germ. ochreata Germ. brunneata Germ. teftata Georg. corrigata Ital. inmorata L. Germ. extremiata Gerist. fcutata Germ. umbilicata Am. m. immutata L. Eur. opicata.S. $N$. remutata Dan. regenerata $A m$. $m$. ofleata Germ. albulata Germ. naffata Germ.

I. 2

Phalti. 
PHALAENA. pufillata Germ.

minutata Germ. nitata Cajen. variegata Germ. diaphanata Sierr. Leon. Lacerata Afr. cingulata L. Eur. niveata Amer. coltata Amer. Inf. ftictiata $A f r$. capitata Ind. aefluata $L$. Ind. argentata Chin. melata $A m$. $b$. fulphurata Cajen. cerata $A m$. m. finuata Afr. aeq. gilvata Ind. Or. violata Tranqb. marinata Ind. Or. urticata $L_{\text {. }}$ Eur. balteata S. $N$. perfpicata Cajem: hifpidata Germ. funerata Suvin. B.) Vfius $C$. fecurata Corom. corneata Anv. Inf. limbata L. Eur. . metallata Afr. aeq. annulata Ind. Or. repandata Germ. verbafcata Germ. geminata Am. $m$. hyalinata $L$. Am. Af. paludata Angl.
PH A L A EN A. potamogata L. Eur.

Aratiolata L. Eur. paluftrata Gall. nympheata $L$. Eur. uligiriata Dan. inundata $A m$. lemnata $L$. Eur. fambucata Germ. candidata Germ. cribrata Ind. $O r$. perfpectata Ind. Or. vitrata Tranqb. exultatis S. $N$. elevata $A m$. m. velotata In]. Rotterd. . 3 punctata Ain. n. vittata $A m$. $m$. revidata Tranqb. indicata $A m$. $m$. derogata Ind. Or. ejectata Ind. Or. feneflata $A m$. Inf. difcata Am. Inf. *** alis forficatis. marginalis Eur. farinalis L. Eqr. B) glaucinalis L. Eur. barbalis $L$. Eur. antennalis Germ. ventilabris Germ. bombycalis Gevm. tentacularis L. Eur.

Phalae.

g) Der Polivfiligel. Der Mobljpanner. La Phalene à ven. tre relevé. 
Phalaena. probolcidalis Phataena, cornicalis

L. Eur.

enfalis Germ.

elongalis Tranqb. lancealis $A m$. In $\int$. roftralis $L$. Eurr. ')

nemoralis Angl.

craffalis Germ.

alpeftralis Germ.

palpalis Angl.

Arigalis $S$. $N$.

poirectalis Am. Inf.

forficalis $L$. Eur.

fophialis Germ.

triftalis Ital.

variegalis Gerns.

dimidialis Ind. Or.

obliqualis Am. m. Inf.

diagonalis Am. m. Inf.

motitalis $\operatorname{Inf}$. Rott.

medialis Am. m.

duplicalis Am. Inz.

undal is Ital.

margaritalis Germ.

fericealis $I n d$.

trinalis Germ.

verticalis $L$. Eurr.

glabralis Germ.

teftalis ' Ind. Or.

falicalis Eur.

alpinalis Gerno.

punctalis Germ.

nitidalis Germ.

obfoletalis Ital.

h) Der Hopfenzünfler. Le Toupet aे pointes.
Ind. Or.

capitalis Tranqb.

dorfalis Braf.

pinguinalis L. Enes.

ramalis Germ. cineralis $l t a l$. aenealis Germ. grifeal is Ital. palealis Gerin. ochrealis Germ. ferrugalis $A f r$. aeq. fecalis $L$.

2 punctalis $A m$. Inf.

bifidalis Am. Inf.

nivealis Germ.

elatalis Germ.

centralis Am. m.

pictoralis Germ.

pudoralis Germ.

flivalis Germ.'

marmoralis Ind. Or.

orientalis.

meritalis Gall. polygonalis Germ.

Janguinalis Ezr.

fticticalis L: Eurr.

poralis Ital.

fafcialis $N$. Holl.

fimbrialis Inf. Amft.

politalis Germ.

repandalis Ind. Or. erofalis Gall.

Atrigalis Germ. recurvalis Am. Inf.

B) angultalis $M$.

L 3

Phalae. 
PHALAENA. erigalis Suec. curtalis Germ. cefpitalis Gorm. facharalis $A m, m$. fluctualis Inf. Rotterd. purpuralis L. Eur. porphyralis Germ. punicalis Germ. minialis. apicalis Strin. . 4 punctalis Germ. argentalis Paedew. collalis Angl. nigralis Ifal. atral is L. Eur. pollinalis Germ. dentalis Ind. Or. rofalis Barb.

minutais Ant Tinf. 437. velutalis Am. Jnf.

Pýalis. (Zïnfles. Pin. cenvs. Tortrices L.)

Bankiana Angl. Lecaina Angl. lumana Ind. $\mathrm{Or}$. prafinaria L. Eur. ') fagana Eur. ${ }^{\text {) }}$ fylvana Erur. virictana L. Eur. ') clorana $L$. Eur. de.

i) La Chappe vert à ban.

k) Der Bucbenwickler. La Phalene verd ondice.

I) La Chappe vere.
Pyralis, vernana Germ. Vahliana Germ. candidana Gall. 6 punctata lnd. Or. uncana L. Eur. plumbana Germ. caprana S. $N$. fponfana Germ. flavana S. $N$. Hattorfiang Germ. orbana S. $N$. Hybnerana Germ. Hermanniana Germ. inundana Germ. roluna L. Eur. aurana Ital. triflana Ital. Burmanniana Surin. Pasquayana Germ. 3 falciana Ital. vitana Gall. pallidana Germ. Sineathmanniana Angl. cretana Grrm. lactana Georg. decuffalsa Germ. 2 punctuna Ital. ameriana L. Eur. maculana Suec. fufcana $L$. Eur.

B) Branderiana L.

Hartmanniana L. Eur'. Forfterana Angl. Pillerana Germ. Saldonana Gall. coftana Dan. 
P×RALIs. Xylofteana L. Eur. miniftrana L. Eur. Snidriana Germ. fervana Germ. lacvigana Germ. Pflugiana' $D$ an. Vdimanniana L. Eur. Solandrana L. Eur. rufticana Gall. variegana Germ. trapezana $D a n$. lufcana Germo. pupillana $L$. Angl. Stroemiana Germ. Zoegana L. Eur. cruciana $L$. Eur. Kaekritziana L. Eutr. Viburnana Germ. cinerana Germb. triangulana Amb. [ns . margana $A m$. In $\int$. obliquana Angl. laetana Susc. profundana Germ. Brynnichatua L. Eur. Iaquiniana Germs. populana Suec. Rolliana Germ. communana Ital. Trauniana Germ. Woelerana Germ. arcuana $L$. Eur. Lecleana L. Eur. Chriftiernapa $L$. Eur. Rohrana S, $N$.
Px ALIs. Buoliana

Germ.

Corylana Germ. Avellana L. Eur. falciana Gall. Haftiana L. Eur. decimana Germs. Baumanniana Germ. Heifeana Germ. Allionana Ital. variana Germ. Lediana L. Eur. Schalleriana L. Eur. rufana Germ. Badicrana Am. Inf. Roborana Dan. laterana Seland. Modecriana L. Eur. bov. Schreberiana L. Eur. Holmiana L. Eur. Francillana Angl. Rolandriana L. Eur. grileana An. m. Inf. gnomana L. Eur. operana L. Eur. Gerningana Germ. ilicana Angl. rivagana Germ. confperfana Germ. Arigulana Germ. Steinkcl nerana Germ. pofticana Gall. Logiana L. Eur. Govana L. Eirr. niveana Suec. criftana Germ.

L. 4 
PYRALIS. Desfontainanía PYRALIS. Alfrömiana

Angl.

profanana Angl.

Thunbergana Germ, punçulana Gervu. afperana Germ, elevana Germ. Bofcand Gall. duplana Germ. fquamana Germ. Romanana Germ. borana Suec. ficticana Angl. litterana $L$. Germ. Junana $S . N$. epipluana S. $N$. liberana S. $N$. emargana Angl. caudana Angl. fcabrana Angl. quercana Germ. Grotiana Germ. Paykulliaua Suec. $\mu h$. Tinz ramella $L_{\text {* }}$ vcellana Angl. Forlkahliana L. Eur. Loeflingiana L. Eur. fulphurana Amb. centraua Gall.

Bẹgutuanmiana L. Eut. Daldorfanna Gcrm. Yeatiana Angl. Schnnuacheran: Dann. Abildgaardan: Dan, mixtana Dan. Penkleriana Germ.
L. Ealr.b. pavoutana Tranqb. Fabriciana L. Eur. nitidana Angl. nigricaula Angl. Hel wigiana Germ. Myllerana Suec. pariana L. Eur. Conwayana Angl. Bierkandraua Sube. abbreviana Dan. Selieftediana Grum. Koenigana Izd. Or. aurana Angl. pomana L. Eur. Schulziana Germ. Lipfiana. rivellana Suec. abietina Sirec. undana Germ. unguicana Ear.

Ph. unguicella L. Scopoliaur Germ. Ifertana Dan, dorfana Germ. Arigaus.

B) Hohenwartiana W. Lundana Germ. Cynosbana Eur. Ph. Cynosbatella $L$. Mygindana Germ. 3 punctana Germ. Mitterbachiana Germ. refinana. Eur. $P h$, refinella $L$. 


$$
G l \circ \iint a t a .
$$

Prralis. mediana Germ. Trnea. perlella Germ.

achatana Gerib.

applana Germ.

Sparmanniana Angl.

Boeberana Dnu.

$P h$. nisella $L$.

umbellana.

Heracleana L. Eurr.

Rutana Gail.

I87. depreflana Succ.

Tinea. (Motteit. Les

Teignes.

boletella Germ.

cerella Eutr.

Ph. cereana $L$.

gigantella Germ.

magnella Ital.

colonella $L$. Eur.

faciella L. Erur.

grandella Ind. Or.

centuriella Germ.

fpificella Germ.

grifella Gall.

gelatella L. Eur.

evonymella $L$. Eur. ${ }^{m}$ )

B) major padella $L$.

\section{Eur.}

plumbella Germs.

fequella $L$. Eur.

nitidella Germ.

cornella Germ.

irrorella $L_{\text {: }}$ Enur.

rofcidella Gernz.

puftulella.

m) Die Spindelbaummotte. Le petit dcuil. linetella Paedem.

$P h$. Tin. rorella $L$.

lutarella L. Eur.

B) unita $W$.

arbuftella Germo

meíomella $L$. Eur.

guttella $E_{n i r}$.

vittella lnd Or.

oxyacantheila $L$. Eur.

vinetella Girm.

argyrella Germ.

Pinetella L. Eur.

pratella L. Eurr.

pafcuella L. Eur.

culmella L. Eur.

margaritella Germ.

conchella Germs.

falfella Germ.

argentella L. Eur.

nivella lnd. Or.

triftella Germ.

carnella L. Germ.

Daphnella Germ.

hortella Lapp.

frigella Germ.

barbella Ital.

marginella Germ.

mucronella Germ.

tefferella Germ.

nigrella Dans.

maurella Gorms.

q̧uadrella ltal.

atrella Itnl.

viduella Lapp.

lugahrella Lapp.

L 5 .

Tinea 
Tinea, variella ltal. anella Germ.

Fagella Germ. cryptella Ind. Or. gramelia Angl. auricella Gall. circulella Germ. Calicella L. Eur. dorfella Cajen. pufiella $L$. Eur. foenella $L$. Eviur. Crataegella L. Eur. palumbella Germ. abietella Germ. tetricella Germb. tapezel!a L. Eur.

B) fufcocephala. pulcliella Dan. infectella.

pellionella $L$. ") veftianella $L$. farcitella $L .0^{\circ}$ ) figmatella Angl. limbella Germ. flavifrontclla Germ. Gall. mellonella L. Eur. cucuilatella L. Eur. arcella Germ. maculella Gall. arhutella $L$. Eur. tefTella L. Eur. Clematella Angl. bicoftella L. Eur. parenthefella J. Eur.

i1) Die Pelzmotze. o) Die Kleidermotre.
Tinea. unialella Ital. elongella $L$. Eur.

- hemedactytell. Germ. olcella.

cylindrella Germ. monachella Ital. (nec frigella). finuella Ital. punctella $L$. undella Germ. cinctella L. Eur. funerella Germ. leucatella $L$. Eur. bifafciella Dan. Arobilella L. Eur. friatella $G$ irm. pectinella Surc. 2 punctella Germ. 4 punctella Germ. 10 guttella Gall. 8 punctella Germs. dodecella L. Eur. viridella Angl. antenella ltal. combrella L. Eur. obfcurelia Sutec. fufcella $L$. Eur. 3 punctelia Germ. 6 punctella Ital. lacteella $A m$. $n$. cinerella L. Etur. bracteella L. Eur. cufpidel la Gerno. dimidiella Germ. trigonella L. Eur. mufcalella Germ. 
Tinea. rhomboidella $L$.

Eur.

gemmella $L$. Eur.

capitella L. Angl. rupella Germ. praelatella Germ. fulphurella Germ. compofitella Angl. miuutella L. Eur. fepulchrclla $A m$. Oliviella Gall. Petiverella L. Eur. fulvella Gism. Lifterella L. Eur. aleella Angl. Knochella Germ. Fyeslella Germ. Geoffroyella L. Eur. Fabriciella Ind.Or. Denifella Germ. Mouffetella L. Eur. Albinella L. Eur.

Seppella Angl. Efperella $A f r$. a $e q$. Goedartella L. Eur. Merianella L. Eur. Allionella Ital. Wilkella L. Eur. SchieffermyllerellaGerm. Bergftraetfella Germ. Schulzella Am. m. Lyonetella $L$. Eur. Pounetclla L. Eur. Schaefferella $L$. Eurr. Scopolella L. Eur. Stroemella Norro. Gleichella Angl.
Tinea. cydoniella Germ. Sparmanella Gall. Rhediella L. Eur. Hermannella Geriw. Roefella L. Eurr.

Leuwenliockella L. Eur.

Myllcrella Eur. Linneella $L$. Eutr. Rajella $L$. Evw. Schreberella Angl. Klemannella Germ. Thunbergella Gall. Harrifella L. Eur. Drurella Danz.

Cramerella Angl. hortella Gall.

Blancardella Angl. Forferella Germ. Syringella Germs. fibulella Germ. exiguella Germ. aurella Germ. Clerkella L. Eur. 182. Lichenella $L$. Eur. ${ }^{\text {p) }}$ ) Alvcita.

Xyloftella L. Esur. B) fifimbrella $W$. capitella Am. Inf. ephippella Eur. dentella Eur. harnella S. $N$. fylvella L. Suec. nemorella Gall. Jucella Suec.

ALv.

p) Porro: T. paralldla et $T$. triangulella. 
Acvcrt $A$. alpella Germ. flavella Germ. vittella L. Ger'm。 nyctemerella Gern. maculella Germ. marginella Angl. 2 punctella Germ. granella L. Eur. q)

Betulinella Suec. nivella Angl. lappella L.'Eur. Perficella. variella Germ. afperella L. Eur. dorfella Germ. coftella Angl. Colutella Gall. lacteella Harb. mediella Germ. fcabrella L. Eur. ariftella L. Eur. caudella L. Eur. Clairvinella S. $N$. Schellenbergella S. $N$. - ' Helwigella S. $N$. Enzenbergella Germ. Swammerdanmella $\boldsymbol{L}$. Eur.

Panzerella Ital. pilella Germ. Robertella L. Eur. Frifcliella L. Eur. Erxlebella Crern. Calthella $L$. Eur. l'ayculella Paedrm.

q) Der waifse Kormzburm;
A L v C I т A. Hybnerella S. $N$. promulella Germ. Degeerclla L. Evr. ${ }^{3}$ )

B) ftriatella $M$. Sulzella L. Eur. Podella L. Etrr. vindella Germ. Reaumurella L. Eur. cuprella Germ. fafciella Angl.

B) Longicornis. fulphurella Angl. formofella Germ. destella $A m$. $\ln$. parvella $A m$. Ins. minutella Ant. Inf. feflicella Germ. 58. oppofitella Angl. Pterophorvs. (Federmot.

ters. Pterophores.) monodactylus L. Eur. ochrodactylus Germ. didactylus L. Eur. calodactylus Germ. Jeucodactylus $A m$. m. 3 dactylus L. Eur. rhododadylus Germ. tefleradadylus L. Eur. pterodactylus L. Eur. albodactylus Gall. migadactylus Germ. pentadactylus L. Eur. ') I3. hexadactylus L. Eur.

r) La coquille d'or. s) Le Pterophore blanc. 


\section{LASSIS $\mathrm{X}$. \\ $\begin{array}{lllllll}R & Y & N & G & O & T & A\end{array}$}

Os roftro: vagina articulata.

* Roftro inflexo.

20I. Fvlgora. Os roftro elongato: vagina quinque articulata. Antennac breves, capitatae.

202. MEMBRACIs. Labium coneum, elongatum, apice membranaceum, fubulatum. Antennae fubulatae, fronti infertae.

203. Tettigonia. Roftrum vagina biarticulata, gulae inferta. Autennae fetaceae.

204. CicADA. Labium rotundatum, apice fetaceum. Antentae fub oculis infertae.

205. CECROPIS. Labium abbreviatum, truncatum, emarginatum. Antennae fub oculis infertac.

2 I . ACANThia. Roftum fub capitis apice infertum. Labium nullum. Antennae anie oculos infertae.

213. LyGaevs. Roftrum fub capitis apice infertum. Labinm elongatum, fubulatum. Anteunae ante oculos infertae.

213. Crmex. Labium elongatum, fubulatuni, annulatum. Antennie ante oculos infertae.

214. Corfass. Labium elongatmm, fubulatum. Antennae capitis apice infertae.

215. Mrkis. Rofti vagina primo articulo comprefto membranaceo. 'Antennae fetaceac.

216. Gerris. Roftrum fubulatum. Iabium brevilfi. wum, rotundatum. Antennac filiformes, ante actulos infertae. 
209. Ranatra. Rofirum breve, rectum: vagina triarticulata. Labium brevifimium, emarginatum. An1tennae palmato bifidae, fub oculis infertae.

218. Pvlex." Roftrun vagina quinquearticulata. Seta uniza. Laminac duae ovatae roftri bafin tegentes.

2 I 9. ApHrs. Rofrum vagina quinquearticulata. Seta unica. Labium fornicatum. Antemuae fetaceae.

210. Navcoris. Roftrum fubinflexim. Setae tres inaequales. Labium porrectum, rotundatum. $\Lambda \mathrm{n}$ tenuae breviflimae, fub oculis infertae.

207. Sigara. Roftrum brevifimum. Vagina conica, transverfin Atriata, lateribus fpinofa. Antennae brevifimae.

222. THRips. Rofrum obfoletum, intra os abfconditum. Antennae filiformes.

\section{* * Roftro arcuato.}

206. Notonecta. Labium fuperius conicum, elongatum, fubulatum. Antennae fub oculis infertae. 208. Nepa. Labium unllum. Antennae palmato divifae, fub oculis infertae.

217. Redvivis. Antennae fupra oculos infertac.

** Vagina pettorali.

220. Cheкmes. Vagina pectoralis. Setae tres inflexae. Antennae cylindricas.

22x. Coccvs. Seta vaginaque pectoralibus. Antennae filiformes. 


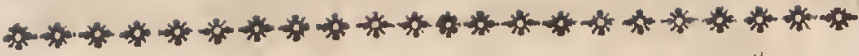

\section{X. $R Y N \in O$ $\mathrm{Y}$ A.}

FVLGORA. (Laternträger. MEMBRACIS. rhombea

La Moucbe Cigale, Alm.m.

Porte-lanterne. The Cicada $L$.

lanthorn - fly.)

laternaria $L . A m . m$.

ferrata Surin.

diadema $L$. Ind.

candelaria $L$. Chin.

phofphorea L. Surin.

noctivida $L$. Surin.

lucernea $L$. Surin.

flammea $L$. Am. ms.

tenebrofa Guin.

obfcurata N. Hol.

adfcendens Cajen.

falciata Cajen.

truncata $L$. Ind.

plana Cajent.

pallipes Cajen.

hyalinata Beng.

feftiva Corom.

feneftrata $A f r$ oeq.

europaca $L$.

minuta Germ.

limbata Germ.

pellucida Germ.

flavefcens Germ.

fronditia $A m$. m.

Cicadia $L$.

foliata Am. m. ${ }^{a}$ )

Cicada $L$.

lunata Cajen.

fafciata Cajen.

bracteata Cajens.

fquamigera Am. m.

Cicada $L$.

haflata $A m$. $m$.

lanceolata Cajerr.

2 maculata Ams. Sept.

fpinofia Inut.

acuminara Penfylv.

rupicapra S. $N$.

planata Ind. Or.

aurita Eur. b)

Cicada $L$.

flexuo!a Ind. Or.

marginata Chins.

horrida Cajens.

trifida Cajen.

clavata Cajen.

MEM.

ftriata Germ.

25. marginata Germ.

Membracis.

inflata Cajer.

a) Cigale feuilletée à ban. de et eire.

b) Le grand Diable. 
ME M B R A C I\$. punctata Braf.

cornuta Eur.

Cicada $L$.

Bubalus .- 2.6 .

Tarandus S. $N$ :

Capra S. $N$.

Taurus Ind. Or.

Vitulus $A m$.

2 punctata $N$. Holl.

Bonafia Am. convoluta Braf. mutica Am. B. inermis $A m$. $B$.

34. Geniltac Angl.

Tetrigunia. grolia Braj: fafciata lav. grilea Amer. limbata Ans. dilatsta Iamaic. fpinofa Sumatra. vaginata Sumatra. 'Tibicen Am. w. Cicada $L_{\text {. }}$

feptemdecin Am* Cicada $L$. variegata Carol. catena C. B. S. maculata Cinin. cantans Barb. aefuans Barb. puftulata $A m . m$. firidula C.B. S.

Cicada $L$.

B) capcifis $L$.
Tettigonia. cingulata.

$$
\text { N. Zel. }
$$

villofa $C . B . S$.

- cruentata $N . Z e l$. confpurcata Ind. heematodes Eur. auffr. Cicada L. plebcja Afr. Ital. Cicada L. Orni Eur. Cicada L. repanda lnid. Cicadia L. -

Kaempferi lap. reticulata $A m$. m. Cicada $L$. picta Galt. Prov. atrata Chin. teftacea Tranqb. flavefcens Guin. olivacea Guin. fanguinolenta Chirs. fplendidula Chin. Ephemera Surin. muta $N . Z e l$. violace:.

'Cicada La

\section{7. minuta.}

CiCAdA. (Singheuf chrecke. La Cigale) perfpicillata Ind. ocellata Tranqb. limbata Afr. aeq. fufcata Tranq b. phalaenoides L. Ans. a punctata L. Am.

Cicada. 
Cicana. retufa Cajert. grifea Am.

3 punctata Afr. aeq. vivida Ann. Inn. minuta N. Holl. marginata L. Am. Inf. pygunaea $A m . n$. Inf. 4 punctata Am. m. InS. cunicularia L. Ind. lanata t. Ind. tomentofa Ind. Or. harbata $N$. Holl. atrata Cajen.

friatula Cajen. nigripennis.

ferruginea C. B.S. undata Carol. irrorata Carol. obtufa Cajen. aurulenta Cajerr. myopa Cajen. villofa Am. m. elongata $N$. Holl. Hiftrio.

futuralis. nigripes $\mathrm{Braf}$. vittata L. Eur. lateralis Eur. ${ }^{c}$ ) variegata $B r a f$. fenefirata $m$. pat. $i$. flavipes InS. Rotterd. interrupta L. Eur. ') lineata Germ.

c) La Cigale à bordure.

d) La Cigale jaune à raies noires obliques.
CicanA. acuminata Gerns. abbreviata Dair. flavicollis L. Eur. viridis L. Eur. ") laeta Cajent. atomaria Ital. prafina ltal. argentata Gall. 4 guttata Cajen. marginella Cajens. lanio L. Earr. Ariata Gall. ignita Cajen. felliva Germ. mixta Gall. bicolor Dan. turca Braf. maura N. Holl. nebulofa Afr. arq. hyalina Ind. Or. pellucida N. Holl. Cynosbatis Dane. clavicornis Gallo. Serratulae Angl. nervufa L. Ear. varia Germ. Jycucea Ind. Or. picta Germ. brunuea Germ. grifea Ital. 2 gutrata Germ. 4 notata Gall. 4 verrucatà Ital.

Crcada.

e) La Cigale verte à tête ponachéc. 
Cicada. fulgida Aighl. diadema Germ. reticulata $A m$. $m$. Inf. punctata Eur. Rolae L. Ewr. maculata Evv". 2 pufulata Girm. aurata $L$. Eur. VImi L. Eur. sriangularis Dan. fplendida (nec nitidula) Germ. virefcens Germ: flavefcens Gerri?. culpidata Ansfl. nitidula Gall.

88. Quercus Germ.

Cercopis.

groffa Afi: aeq. matginata $A m$. w: maculata Afr: aeq. coccinea Am. str. Iirs. rubra Senegal. fanguinolenta L. Eur. ' analis $C . B: S$. obfcura Guiso. Schach Am. Sept. cruentata Suris.

Cicada rusuta $L$. verficolor Iranqb. atra Eur. au/tr. viridis $A m, I n f$. nebulofa' $I n d$. Or. carnifex $N$. Holl.

f) Dic blutrotbgefleckte C: cade. La Cigale à taches rouges.
Cercopis, vatia Cajert. 4 falciata Surin.

Cicada $L$.

fpumaria Eir. ${ }^{8}$ )

Ciiada L. aurata Cajeiz. marginella $D a m$. leucophthahn. Eur.

\section{Cicada $L$.}

Jeucocophala Eur.

Cicada $L$.

firiata Germ.

lineata Germ.

vittata Gall.

coleoptrata Germ. Gall. angulata Suec.

piaculla Suec.

immaculata ltal.

aptera Barb.

grylloides Ital.

pedeftris Paedem.

ruffica Eur.

gibba Dan.

2 guttata Germ.

rulicollis Ital.

variegata.

fafciata Germ.

nuifafciata Ital.

2 fafciata Stzec. ${ }^{\text {h }}$ )

Cicada $L$.

capitata Grll.

3 falciatz Gall.

Cerco-

g) Die Weidenfclarumcicade. Le Cigale bedeaude.

h) Das Dopreliond. Cigale à trois bandes blanches. 


$$
R y \boldsymbol{g} g \text { ot } a \text {. }
$$

Cercopis. friatella Ital. hiftrionica Ital. Populi L. Eur.

Cicada $L$. 46. reticulata Eur.

Notónecta. (Waferwamze. La Punaife d'eau.)

glanca L. Eur. ${ }^{\text {') }}$

furcata Gell. maculata Gall. americana. nivea $I n d$.

6. minutifima L. Eur. ${ }^{k}$ )

Sigara.

lineata Cajen. ftriata Eur.

Notonecia $L$.

B) minor.

coleoptrata Sulec. 4. minuta Gall.

NEPA. (Wafferforpion. Le Scorpion aquatique, la Naucore.)

grandis $L$. Am. annulata Coromo ruflica Corom. fufca $L$. Ind, Or. groffa Chin. rubra $L$. Tranqb.

i) La grande Funaife à avirons.

k) La petite Punaife à avi. tons.
Nepa. cinerea L. Eutr. ${ }^{1}$ )

8. maculata Ind. Or.

RANATRA.

filiformis Tranqb.

linearis Eur. Nepa $L$. elongata Tranqb. cinnicoides Eur. Nepa L. aeftivalis Gall. nepreformis $A m$. mo. 7. curfitans Germ. ACANTHIA.

lectularia Germ. ${ }^{\mathrm{m}}$ )

Cimex $L$. Zofterae Germ. flavipes Gevon. atra Germ. pallicornis Gerns. grylloides Girm. Cinex $L$. coriacea Girm. clavipes Tranqub. nigricornis Germ. clavicornis Eur. ${ }^{\text {") }}$

Cimex $L$.

crafficornis Gern. . virefcens $A m$. m. Inf. Lavaterae Barb. Serratulae Angl. fafciata Gerni. M 2 Acan.

1) Le Se, à corps ovale. m) Bettumanze. Punaife de lit, the Wall loufe-

n) Das Kezllhorn. P. La Punaife tigre. 
A C A N THIA, maculata Trangh. pallipes Date. littoralis Eur.

Cimex $L$. rugofa Am. $b$. Cimex L. lunata Ind, corticalis Eur.

Cinuex $\boldsymbol{L}$. deprefla Gerw. plana Germ. paradoxa $C . B . S$. laevis Angl. Betulae Eur.

Ciniex $t$. grifea Barb. craflipes Germ. ${ }^{\circ}$ ) crola Surin. Cimex $L$. monfrofa $B a r b$. umbraculata Dant. $^{\text {P) }}$ ) ferrata. gibbe Ind. Or. campeftris Seland. nemoralis Seland. partenfis Germ. fylvenris $E_{u r}$. Cinuex $L_{\text {. }}$ alata Sttec. coftata Eur. bur, Sacchari Am. m. Inf.

o) Die dickbeinicbte, Wan2e. $P$. P.

p) Die fchattirte Wanze.
Acanthia. Sidae Am. $m$. Inf.

Cardui.

Cinex L.

Humuli Germs.

Pyri Germ.

45. Gollypii Am. m. Inf.

Crimex. (W anze. La Pu. naife. Bug.)

- Scutellati fcutello longiiudine abdominis.

Stockerus L. Chun.

Eques Tranqb. nobilis $L$. $A$ f. figuatus $S e n e g$. regalis $N$. Holl. imperialis $N$. Holl. difpar Chin. Nigellae Barb. Cerinthe $A f r$. annulus Seneg. 6 punclatus $A m$. $m$. atcuatus $A m .4 m .1 n j$. Drurasi Am. Fabricii L. Cajen. Argus Surin. Scliulzii Cajen. paganus $N$. Holl. illuftris $C . B . S$. furcifer $C . B . S$. lineola $C . B . S$. 3 lineatus $A m$. $m$. nigrolineatus $L$. Eur. auf(r. q)

Crmex.

q) Die Bänderwanze. La Punaife Siamoife. 
Cimex. fernipunctatus An.

fylphoides Ind. Or. anchorago $A m$. grammicus $L$. Afr. paedemontanus. collatus Inful. Rot'terd.

Jynceus Barb. manrus $L$. Or. hottentottus Orient. albolineatus Ital. rufticus Tranqb. irroratus Am. lanatus Sibir. globus Eur, aufir. fcarabaeoides L. Eur. pallipes Afr. aeq. Havipes $N$. Holl. Desfontainii Barb. Vahlii $O r$. fuliginofus $L$. Eur. litura Arab. tuberculatus Ital. inunctus Angl. Arabs L. Ani. Ins. folidus L. Ind. * Spinofi thorace utrin. que Jpina armata.

Taurus Corom.

Cervus Cajer.

Dama Ind. Or.

Vacca Guadel.

Gazella Martiniq.

Tarandus. bidens L. Eur. fanguinipes Ital.
C. 1 MEx. rufipes $L$.

$$
\text { Eur. }{ }^{\mathrm{T}} \text { ) }
$$

luridus Angl. cuftos Germ. nigricornis Germ. nigrifpinus Chins. ictericus $L$. $A m$. punctatus L. Eur. varius $H i f p$. lunula Barb. albipes Ital. dentatus Ind. Or. floridanus $L$. armatus $N$. Holl. haemorrhous L. Am: 2 puftulatus $L$. Surin. punicus $L$. Afr. Ypfilon L. Surin. clypeatus $L$. Chin. clector. albicullis Iamaic. haemorrhoid. L. Esur. fpinidens Tranqueb. Sagitta Am. m. InS. oculatus Chin. annulatus Virg. 4. puftulatus Ams: maculatus $A m$. pugnax $A m$. emeritus N. Holl. : gladiator Am. ferrugator Suec. crenator Ams. Inj. M 3 Crmex:

r) Punaife i pattes rouges: Der Rotufiufs. 
Crmex. furcatus Patag. pugillator $A f r$. perditor Am. Inf. victor $A m$. $\ln \int$. delirator $A m . J n f$. ciliatus $A m$. melacanthus $A f r$ a aq: aggreffor $N$. Holl. vittatus $C$. $B . S$. hamatus Ind. Or. velox Ann.

** Ovati thorace muíico.

aurantius Iava. punctum Iava. nigripes $I n d$. Or. Iferti Guin. papillofus Sierr. Leon. rubens Iitd. Or. Ianus (nee danus) Am. Inf. obfcurus Ind. Or. torquatus Ind. rutilans Afr. aeq. guttatus Siam. viridulus ' $L$. Ind. prafinus L. Eur. ') diffimilis Germ: juniperinus $L$. Eur. fmaragdulus Mader: fpirans S. $N$. azureus Guin. beryllus Ind. Or: calidus Sierr. Leono. lynx Hung.

s) La Punaife verte. Die Grasgrime Bauswanze.
Crmex. coelebs $N$. Holl. iratus Cajen. dumolus L. Eur. 3 punctatus Am. atomarius Ain. nubilus $C . B . S$. triftriatus Pardems. 6 punctatus L.' $7 n d$. fignatus Sierr. Leon. gravis $N$. Zel. meditabundus $A m . m$. cinctus Orient. rubrofafciatus Trangb: feladonius Ind. Or. agathiuus Germ. lituratus Ital. - cruentus Surin. geniculatus Cojen; mixtus Cajent. Picus Ind. Or. tripterus Cajen. grifeus $L$. Esur. interftinctus $L, E u r$. funebris Sierr. Leon. ruficornis $I n d$. baccarum L. Eur. '? viduatus Guin. cribrum Surin: mucoreus Chins. brevicornis Chin. glaucus $N$. Holl. . ornatus' L. Eur." )

Cimex.

t) La Punaife brune aे antennes et bords panachés. choux.

ii) La Punaife rouge du 
CrMex: feflivus L., Cimex. I80; acuminatus

Enir.

rutilus N. Holl.

cruciatus $\operatorname{lnd}$. Or.

difcoideus Cajen.

verficolor Giin.

fpliacelatus Germ:

bioculatus $A m$ :

gramineus Tranqb.

fimbriatus Chin.

2 punctatus Ital. bicolor L. Eur. ") olerąceus L. Ear. "') pictus Ind. Or. 2 guttatus L. Ear. Hillrio Tranqb. coeruleus L. Eutr. ${ }^{\mathrm{x}}$ ) albomarginellus, G $76 \mathrm{rm}$. albomargiratus Germ. -nigrita Gern. flavicornis Germ....? - Mório L. Eura - - i triflis $O r .{ }^{y}$ )

Cpinipes Afr, aeq. aethiops Cajens. lugens $A m$. melanoceph. Angl. perlatus Germ. $\therefore$ (i) decrepitus Dama ?ins

v) .La Punaife noire \& quatres taches blanches.

.w) La Punaifé, verteà raies et taches rouges on blanches.

x) La Punaife verte bleustre.

y) La Punaife noir.
L. Eur. ${ }^{z}$ )

Couevs.

narginatus Eur.

Cinex I.

Scapha Gertm.

fpininger Ital.

venator Ital.

bellatol Cajer.

armiger Afrora.

lanciger Guin.

hallator Guin.

fcorhuticus Am. $\ln \int$.

2 guttatus $I m d$. Or.

delirator Cajerz.

defenfor N. Holl.

puguator Tranqb. fafciculatus C. B. S.

infidiator Barb.

caluminiator Ind. Or:

hirticoruis Barb. ?

fulcicornis Barb: rhombeus $A f r$.

Cimex $L$.

quadratus Germ:

laftatus Tranqb.

22. gravidator Ame mi

$$
\text { Inf. }
$$

LYGAEVS.

* Thor. Spinofo.

valgus. C. B. S.

M 4

Cimex

2) La Punaíc à tête allongée. Porro:

1) Cinex chlorizans.

2) - - bifpinus."

3) - melanosephalus. 
Cinzex $L$ :

LYGAEv s. ferratus $A m$.

Merianae Surin.

groflipes Tranqb.

tragus Ch́in.

tenebrofus Ind. Or.

fulvicornis Ind. Or.

incubitor Carol.

heros Ind. Or.

femoratus Ind."

curvipes Afr. aeq.

clavipes Chin.

dentator Ital.

pictor Ind. Or.

compreficorí. Cajen.

menbranaceus Prom. $v$. phýllopus Amo auftr.

Cimex $L$.

gonagra Am. Ins:-

kermefinus Surin.

Cimex $L$.

1 auctus Am.

auftralis.

cruciger Braf.

fanctus $B r a f$.

laetus Cajen.

Juhatus Cajent. elatus Cajen.

balteatus $A m{ }^{2} m$.

Ciniex $L$.

venofus Cajon:

finuatus Cajen.

ferripes $N$. Holl.

dentipes Sierr. Leoon.

linearis Chin.

crenulatus Am. Inz.
LYGAEVS. 4 fpinofus Ann.

Cimex $L$.

* Thor. mutico.

phafianus $A f r$, aeq.

beilicofus Afr. aeq.

meleagris Chin.

gallus Surin.

faber Poulicand.

harpagon Am. indus L. Cajen.

Cimex $L$.

militaris Orjent.

equentris $L$. Eur.

Cimex $L$.

civilis Trangh.

crudelis C. B. is.

"Faxatilis Eur.

- Cinex $L$.

Hyofcyami L. Eur. familiaris Eur.

varicolor $\operatorname{Inf}$. Triwit.

hofpes Tranqb.

abbreviatus Ind. Or.

roftratus Afr. aeq.

malabaricus.

aulicus Ams.

varicornis Ind. Or.

rufo inarginat. Ind. Or.

Boerhaviae Guin.

pallens Afr. aeq.

errans Barb.

cingulatus $N$. Holl.

fuperftitiofus $A m$.

albofafciatus Sarrin.

ruficollis Braf:

LYGAE. 
Ly GaEv S. fabrofus Ans.

leprofus $A m$.

leucurus $\ln \int$. . Amft. Koenigii Tranqb. Slanbufchii Chin. aegyptius.

Cimex $L$.

fanguineus Ind. mendicus Ind. fervus Chin. fanelicus $C$. B. S. trilineatus Ind. punctum Gall. 4 guttatus N. Holl. punct. guttat. Ital. 2 guttatus $N$. Holl. fanguiuolent. $A m$. 6 punctatus Hifp. cruentus Jind. Or. futuralis $A m$. m. pulchellus Inf. St. Cr. rubiginolus C. B.S. excavatus $A f r$. aeq. furcatus Guins. carnifex C. B. S. augur Ind. Or. maclans Int. Or. apterus $L$. Eur. Cinsex $L$. ftriatulus Braf. guttula Am. Inj. nugax Ital. calcaratus L. Eur. lineola An. Inj: .
LyGAEvs. maroceanus.

Pini Eur.

Cinex $L$.

Sylvaticus Suec.

Rolandri L. Eur.

Cinex $L$.

Forlteri C. B. S. fordidus Tranqb. armipes. lufcus Germ. clavimanus Afr. aeq. 6 puftulatus Iamaic. Vrticae Angl. lynceus Angl. fylveftris Dan. erraticus Germ. lunatus Cajen. podagricus Angl. magnicornis Barb: Chiragra Dans. crafficornis Eut. Cimex $L$. hyalinus Ant. 1nf. Sidae $A m$. $m$. clavicornis Dan. capitatus Germ. arenarius Eur.

Cimex $L$. fuccinctus $P_{e n z}$ ylu.

Cimex $L$. tunicatus Germ. pubicornis Am. Coryli Eur. Cimex $L$. nemoralis Barb. M 5 
LYGAEvS. lineatus S. $N$.

campettis Eur.

Cinex $L$.

pratenfis Eur.

Cimex $L$.

floralis Dans.

4 punctatus Germ.

2 punctatus Norw.

2 notatus Sule.

Froxini Germ.

ferrugatus Germ.

ftriatellus Germ.

inquinatus Germ.

bis 3 guttatus Germ.

naffates $D a n$.

Tiliae Gurm.

Populi Eur.

Cimex L.

6 guttatus Eur.

leucocephalus Easr.

Cimex $L$.

arbuftorum Seland.

transverfalis Germo.

Alux Dan. rofeus $\mathrm{M}$.' fanguinens Germo :

3 puftulatus Dan.

3 guttatus Germ.

Cimex $L$.

faltatorius Eur.

Cimex $L$.

elatus Germs.

ater Eur.

Cinzex L.
LYGAEV́s. tyranuus

Ital.

fchach Ital:

2 fafciatus Germ.

C. clavatus $L$.

unifafciatus Danz.

Havovarius Dan. umbratilis Suec.

Cinsex $L$.

flavicullis Angl.

ruficollis Barb.

3 fafciatus Eur.

Cinex $L$.

olivaceus Germ.

feticornis Germ.

capillaris Germo.

gothicus Eur.

Cimex $L$.

fcutellaris Germ.

albomarginatus Gall.

danicus.

palliatus'Barb.

3 color Dan.

tipificornis Germ:

flavounaculat. Gernz.

agilis Germ.

fcriptus Gall.

hyalinatus Ital.

I 75. varius Germ:

Mikis.

dolabratus Eur.

Cimex L.

laevigatus Eur.

Cinex $L$.

lateralis Germo. 
Miris. holfatus. pabulinus Eus.

Cinuex $L$. virens Eur.

Cimex $L$. pallens Suec. calens Ind. Cimex $L$.

Gronovii Ind. Cimex L. ferus Eur.

Cimax L. vagans Susc. cingulatus Ind. Or. marginellus ltal. ftriatus. Eur.

Cimex $L$.

Abictis Eur.

Cimex $L$.

16. Vlmi Eur.

Cinsex $L$.

GERRIS.

lacuftis Eur. ")

Cimex $L$.

paludun Gall.

foflarum Ind. Or:

ftagnorum Angl.

Cimex $L$.

rivulorum Alfat.

pallipes Ital.

calciformis Ams.

Mantis.

tipuliformis Guin.

a) Die Teichmanze. La Ptse naife nayade.
GERRIS. praecatorius

Gritin.

pedeftris $1 n d$. Or. filiformis $A m$. oratorius Ind. Or: anguftatus. Chine. Filum Ind. Or. longipes Am. curfitans $N$. Holl, tipularius Eur. Cinnex $L$. vagabundus Eur:

Cimex $L$. clavipes Suec: apterus $1 t a l$. 22. currens Ital.

REDVVIVS.

gigas Ind. Or. perfonatus Eur. ${ }^{b}$ )

Cimex $L$.

villofus Barb.

barbicornis Sierr. Leon;

maculatus Afr. aeq.

ftridulus Gall.

nitidulus $A f r$ a aq. pilipes Cajen. Jongipes Am. InS.

Cimex $L$.

Phalangiun Am. Inf. nigripennis Ind. Or. marginatus $I n d . O r$. rufipes Cajen.

REDV:

b) Die . Flingenvanzze. La Pแnaife nuouche. 
RE D v I vs. 2 puftulatus Surin.

fanctus Ind. annulatus Etur. Cinex $L$. fanguineu's Barb. cruentus Gall. moeftus Guadel. cinctus $A m . b$. bicolor "Afr. aeq. litura Cajeu. atteläboides $N$. Holl. pilicornis Tranqb. quadridens $A f r$. ueq. nigripes $A m$. $m$. Cimex $L$.

hirtipes Cajen. haemorthoid. Barb. lineola Cajen. Aria Cajen. collaris Iud. Or. varius $N$. Holl. 6 guttatus Ind. 4 guttatus Ind. Or. punctum Ind. Or. 2 punctatus $I n d$. Or. hąmatus Cajen. iracundus Idriac. fulcipes $I_{n d}$. Or. bifidus Chin.

2 guttatus Guin. Cimex $L$. ferratus $A n$. Cimex $L$. albipes Eur.
REDvvivs. aegyptius. leucocephalus Guin. diadema Am bor. 5 fpinofus $1 n d$. Or. 4 fpinofus Cajen. fcutellatis Cojen. fafciatus Cajelz. maurus Maurit. Acantháris Iamraic. Cimex $L$. fornicatus Cajen. guttula Germ. elougatus Afr. aeq. 56. minutus Gall. Pvlex. (Floh, Puee, Flea.)

irritans Eur. Anu. penetrans L. Am. m. Arnis. (balttlaus. La Pu. ceron. Plantlonse.) longiroftris Germ. Lyclnidis $L$.

Capreae.

Sambuci $L$.

Solidaginis.

Cerafi.

Ribis $L$.

Arundinis. burfaria $L$. Aparines. $\Lambda$ ceris $L$. Rumicis $L$. Paftinacae $L$. Pruni. Achilleae. 
Apris. Cardui $L$.

Millefolii.

Nymplieae L.

Corni.

Abriuthii $L$.

Evonyui.

Avenae.

Fraxini.

Fagi $L$.

Betulae L.

Alni.

Populi $L$.

Viburni.

Mali.

Rofae $L$.

Atriplicis.

Picridis.

Aegopodii.

Dauci.

Vrticae.

Tanaceti $L$.

Vimi $L$.

Papaveris.

Tiliae $L$.

Iuniperi.

Brallicac $L$.

Roboris $L$.

Quercus L. Gail.

Pini $L$.

Pineti.

Liguftici.

Salicis $L$.

Piftaciae $L$.

Acetolae $L$.

Padi $L$.
$\Lambda$ PHIs. Viciae $L$.

Lactucae $L$.

Souchi $L$.

54. Vitis.

Chermes. (Blattfloh. Blattfauger. Le Kermes.)

Graminis $L$.

VIni $L$.

Pyri $L$.

Ceraftii $L$.

Buxi $L$.

Fagi $L$.

Sorbi tho

Perficae.

Calthae $L$.

Vrticae $L$.

Betulae $L$.

Alni $L$.

Abietis $L$.

Salicis $L$.

Fraxini $L$.

Aceris $L$.

17. Ficus $L$.

Coccvs. (Schildalaus. La

Cochenille.)

Hefperidum $L .^{\circ}$ )

Aonidum L. Afiae.

capenfis $L$.

Adonidum $L$. Eur.

Quercus $L$.

Ilicis L. Gall. ')

Coc*

c) Le Kermes des Orangers.

d) Lon verneon efpelis. 
Coccvs.- Ficus Ind. Or.

Caricae Gall. Aceris Gall. VImi $L$.

Coryli $L$. Betulae $L$. Carpini $L$. Tiliae $L$. Rufci $L$. Myricae $L$. Capreae $L$. Pilofellae $L$. Arbuti $L$. Phalaridis $L$. Crategi $L$. Serratulac.
Coccvs. Vitis $L$.

Zofterae L. Mav. Balt. polonicus $L .{ }^{\circ}$ ) Cacti L. Am. ${ }^{\mathfrak{f}}$ )

27. dubius ltal.

Thrips (Blafenfufß. L L Thrips.)

phylapus $L$.

Iuniperina $L$.

Vlini.

Vrticae.

falciata $L$.

6. minutifima L. Eur.

e) Le Kermes dez racines.

f) Cochenille mefteque. The cochineal Fly. 


\section{L A S S IS [XI.}

\section{A. $N$ T L I A T.A.}

Os probofidte atque hniftello.

225. MYDAs. Haunellum feta unica absque vagina et palpis. Antemac approximatae: clava ovata, comprefta.

224. Tipvas. Hauftellum absque vagina. Palpi duo porrecii, filiformes ad bafin proboscidis.

23r. Ceria. Hauftellum univalve feta unica. Palpi duo capitati, niedio proboscidis inferti. Antennae ciavato acuminatae, petiolo communi infidentes.

129. Nemoteld, Hs. Hauflellum feta unica recurva absque vagina et palpis. Antennae articulo ultimo longiori, fetacco bafiu proboscidis infertae.

226. Bis:o. Haufellum fetis tribus vaginaque univalvi. Paipi brevillimi.

228. Stratiomys. Hanfellun feta unica absque vagina. Palpi breves, fubcapitati.

230. Rhagro. Haufellum fetis rribus absque vagina. Palpi duo pilufi, porrecti ad bafu hanfelli.

238. SYRruvs. Hauftellum fètis quatuor absque vagina. Palpi dno bafi fetarum exteriorum inferti.

233. Mvsca. Haufellum feta unica absque vagina. Palpi dno porrecti, extrorfum crafliores.

234. Tabanvs. Hauftellum vagina univalvi fetisque quinque. Palpi duo clavato acuminati.

235. Rhingia. Hauftellum vagina univalvi, os obtegente fetisque quatuor. Palpi filiformes.

237. Conops. Hauftellum vagina univalvi, abbreviata fetaque unica. Proboscis porrecta ultra bafin geniculata. 
24I. Empis. Haufellum et probofeis inflexa. Hau. ftellum vagina univalvi fetisque tribus. Palpi bre. ves, filiformes.

*:* Os hanftello absque probofcide.

223. Oestrvs. Hauftellum retractum intra labia con. nata, poro pertufa.

236. Asinvs. Haufellum rectum, bivalve, hafi gibbum.

238. Șтомохxs. Haunellum vagina univalvi, convoluta, bafi geniculaca Cetisque duabus vaginantibus.

227. ANTHRax. Haufellum bivalue: valuula fuperiori breviori. Palpi duo elongati, filiformes, haufelli bafi inferti. Antenuae diftantes.

239. Mrop A. Hauftellum bafi medioque geniculatum. Vagina bivalvis; valvulis inaequalibus.

240. Cvrex. Vagina exferta, univalvis, flexilis: fetis quinque. Palpi duo triarticulati.

242. Bombysivs. Hauftellum longiflimum, rectum, fetaceum, hivalve. Antennae bafi connatae.

243. Vouvccella. Haufellum elongatum, rectum, fetaceum, bivalve absque palpis. Setae tres incum. bentes, aequales. Sntennae approximatae: articulo ultino cylindrico, obtufo.

244. Cytherea. Hauflilum rectum, porrectum, fetaceum bivalve. Antennae difintes: articulo ultimo fubulato.

245. Ниртоволса. Haufellum breve, cylindricum, rectum, bivalve: valvulis aequalibus.

246. Pycnogonvm. Hauftellum tubulofum, conicum absque fetis. Palpi duo ad bafin hauftelli.

248. PEDicvivs. Hauftellum retractile, recurvum.

249. Acarvs. Hauffellum vagina bivalvi, cylindrica. Palpi duo longitudine liauftelli.

247. Nympнon. Hauftellum tubulofum, cylindricum. obtufun. Palpi quatuor ad bafin hauftelli: fuperioribus chelatis. 


\section{A N T L I A T A.}

Oestrvs. (Bremele. L'oe- Tipvla, pratenfis L. Eur.

ftre. Gad. Fly.)

buccatus Carol. a.

pecorum.

Bovis $I$.

vituli $L$.

Tarandi $L$.

Trompe Lapp.

Equi.

B) haemorrh. L.

8. Ovis $L$.

Tipvla. (Schnacke. La

Tipule. Crane - Flty.)

* alis patentibus. pectinicornis $L$. Eur. rivofa $L$. Eur.

finuata Dan.

4 maculata L. Eutr.

crocata L. Eur.

flaveolata Germ.

hortorum $L_{0}$ Eur.

oleracea $L$. ")

tricolor Am. b.

triangularis Scotias.

variegata $L$. Eutr.

contaminara L. Eutr.

lunata $L$. Evtr.

turcica Gern. brutis. dorfalis Germ. Hiftro Dan.

verticillata $D a n$.

plumbea Ital.

Ariata Ital.

cornicina L. Eur.

atrata $L$. Eur, $b$.

nigra $L$, Eatr.

albimana Germ.

clavipes $A m$. $b$. paludola Ital.

lacta Ind. Or.

2 maculata $L$. Eurr.

annulata L. Fur.

ocellaris L. Eur.

cinerea Nomo.

fafciata $L$. Succ.

melanoceph. Cajen.

6 punctata Ital.

thavipes Germ.

3 punctata Ital.

flavefcens Esur.

regelationis L. Eur.

pilipes Germ.

Morio Germ.

replicata $L$. Eur. 6. monoptera $L$. Eur. ** alis incumbentibus. plumofa L. Eurr. 
TIPVL A. motitatix $L$. TIPveA. Chryfanthemi

Eur.

pilicornis Germ.

fafciculata Germ.

tendens Diar.

tremula $L$. Suec.

flexilis L. Eur.

vibratoria L. Eur.

Hintrio Gernn.

varia Germ.

rufipes L. Dan.

gibba Germ.

fricta Germ.

pallida Germ.

barbicornis L. Eur.

plumicornis Dan.

Habellicornis Germ. cincta $L$. Suec.

Arundineti $L$.

littoralis L. Eur.

monilis L. Eur.

zonata Angl.

macroceph. $L$.

viriclula L. Eur.

cantaus Germ.

geniculata Girm.

pallipes Germ.

pufilla $L$. Eur.

brevicornis L. Eur.

hortulaua $L$, b)

B) Marci L.

Iohannis L. Eur.

Poinonae Angl.

Pyri Germ.

ruficollis C. B.S.
Hifp.

Thomae L. VPS.

febrilis L. Eur.

bruanipes Terr. $\boldsymbol{n}$.

forcipata Angl.

albipenn. Dan.

juniperina $L$.

hirta $L$.

phalienoides L. Eur.

Perficariae $L$.

culiciformis $V p$ s.

paluftris Eur.

lylvellis Germ.

longicornis L. Eur.

fericea $L$. succ.

pulicaris L. Eur.

93. pennicornis $L$.

Mydas.

filatus Am. auftr.

illucens $A m$. m. Iss. $M u u f c a$ L.

2 lineatus $N . Z \% l$.

Bibio.

aenea Germ.

pleiseja Eur.

$M u f c a L$.

flavipes Germ.

marginata Ital.

nobilitata Dam.

lugubris Germ.

ftrigata. Germ.

florea Germ.

9. anilis L. Eur.

Mufea $L$.

b) Le Bibion de St, Marc. ANThrax. (Motrenfliege.) 
Anthrax. Cerberus Am.m. 'Stкatiomss. fplendens

nigrita Am. Ital.

hetrufica 1 tal.

Lar Bpngal.

Morio Eur. ')

Muifá Lo.

nuaura Eur.

$M u \int c a L$.

ofra Germ.

algira.

holofericea Barb.

varia Gall.

aethiops Ital.

capucina $A m$. $b$.

Satyrus $N$. Holl.

Belzebul Hung.

Tantalus Tranqb.

lottentotta Eur.

$M u \int c a L$.

Sylvanus $N$. Holl.

Ixion Ital.

Sabaeus Ital.

Sphinx Ind. Or.

Lucifer Am. Inf.

Troglodita Ind. Or.

23. Abbadon Ital.

SтR А т I M Y S. (W W affen.

firge.)

teftacea $A$ m. m.

errans.

bicolor S. $N$.

chanueleon Eur. d)

Minfa L.

e) La Mouche à ailes noires bordées de blane ondé.

d) La Mouche armée à ven. tre plat, chargée de fix lunules.
Hijp.

furcara Germ.

Ephippium Eur. ')

crucis $A m$. m. Inf.

flaviffima 1tal.

Mictoleon Eur,

$M u f c a L$.

Arigata Ital.

.cyanea Cajen.

pallipes $A m . b$.

mutabilis Cujen.

fafciata Cajen.

argentáta Germ.

tigrina Dan.

Hydroleou Eur. ')

$M u f c a L$.

viridula Danz.

trilineata Eutr. ${ }^{\text {B) }}$

$M u$ fa $L$.

Hypoleon Eur. ')

Muesa $L$.

mufcaria Ital.

minuta Tranqb.

4 lineata Sirrr. Leon. . clavipes Suec. ')

Mufca L. .

$N_{2}$

STRA-

c) La Mouche armée à corcelet rouge fatiné.

f) La Mouche armée à ven. tre verte.

g) La Mouche armée jaune à bandes noires.

h) La Mouche armée noire. \& taches jaunes.

i) La Mouche àrınée noire خे ventre et cuiffer jaunes. 
Stratiomys. 26. 6 denta. ta Angl. ")

Nemotelvs.

uliginofus Germ. Musca $L$.

villofus Hung.

marginatus Angl.

punclatus Barb.

5. pufillus Tranqb.

RHA GIO. (Schnepfenflio

$$
\text { ge.) }
$$

fcolopaceus Eur.

$$
M u f c a L \text {. }
$$

tringarius Eur.

$M u f c a L$.

vanellus Dan.

ardea Ital.

Vermileo Gall. Musca $L$.

lineatus Angl.

fufcatus Suec.

falciatus Norw.

tipuliformis Germ.

flavipes Germ.

bicolor Ital.

maculatus Gern.

punctatus Germ.

Iongicornis Germ.

cinctus, Germ.

tomentofus $D$ an.

lineola Dan.

k) Porro:

I) Stratiourys macro. leon. $\mathrm{P}$.

3) - conica. $P$.

2) $\bar{P}$. unguiculatn.
Rhagio. Morio Dan. nigrita Ital. Diadema Germ. $M u r$ ca L. atratus ltal.

22. colombafchenfis Serv.

Ceria.

clavicornis Germ. Barb.

SY R P Y S. (Einfchuitt-

fiege.)

* Antenmis plumatis. inanis Ear. Mufca L. micans Ital. pellucens Eur. ') $M u f c a L$. bombylans Eur. . $M u \int c a L$. myltaceus Eur. Musca L. muflitans Gomm. Lapponum. $M u f c a L$. inflatus Ital. apiarius Ital. efuriens $A m$. $I n j$. intricarius Eur. Mufca L. crallus Tranqb. bombyliformis Germ. vacuus $A m$. Inf. obefus Amr.

* Antennis Seta muda. pinguis $A m$.

SYRRHVS.

1) La Volucelle à ventre blanc en devanc. 
S X R P K V s. pendulus

$$
\text { Eur. }{ }^{\mathrm{m}} \text { ) }
$$

Musca L.

natans Ital.

verficolor Germ.

floreus Eur.

$$
\text { Mujea } L \text {. }
$$

fubcoleoptratus Stuec.

$$
\text { Conops } L \text {. }
$$

hemipterus Angl. craflipennis Gall. affinis Gall. nemorum Eur.

$$
\text { Mufra } L \text {. }
$$

cryptarum Germ. zgrorum $\Lambda \mathrm{m} . \operatorname{In}$. vinetorum $\mathrm{S} . N$. arvorum Chin. hortorum $A n$. $\operatorname{In} \int$. arbuftorum Eur.

$$
\text { Mivfa L. }
$$

pratorum $A m . I n S$. faltuum Ital. frutetorum Angl. ericetorum $A f r$. aeq. murorum Barb. tenax ${ }^{\mathrm{n}}$ )

Majca $L$.

fetofus Barb. fegetum Bnrb.

3 lineatus $N$. Zel. 4 lineatus Tranqb. 5 Atriatus Ind. Or.

m) La Mouche à corcelet frie et bandes jaunes interrompues fur le ventre.

n) La Mouche apiforme,
Sx R PHVs. 5 lineatus $A f r$ aeq. fuciformis Gall. oeftraceus Eur. Mufca $L$. afiliformis Germ. lucorum Eur. $M_{i i f c a} L$. fallax Eur. $M u s c a L$. fylvarum Eur. $M a$ fca $L$. nigitita lamaic. clavipes Ital. equeftris Ital. flavicans Ital. cinereus Ital. 2 cinctus Eur. Mufca $L$. arcuatus Eur. ${ }^{\circ}$ ) $M n \int c a L$. cingulatus $N$. Zel. zonalis Chin. errans Chin. mutabilis $E u r$. $M u$ s $a$ L. devius Evur. $M u \int c a L$. (egnis Eur. ${ }^{\text {D }}$ ) Majca $L$. volvulus Gall. piger Germ. N 3

SrR-

o) La Mouche initante la guêpe, à longues antennes.

p) La Mouche dorće à ventre brun et noir. Munche \&े fegment. ; 
SYRPHVs. lunatus Barb. ifemoratus Eur. Mufica L. fpinipes Goll. anumlatu: Gall. dentipes Cajen. conopleus Eur. $M u s \in a L$.

ichneumoneus Enur. $M u f c a L$ bicolor Barb. fphegeus Angl. clavatus Am. m. cylindricus Amu. Inf. veficulofus $1 \mathrm{tal}$. elongatus Dan. podagricus Dan. crabroniform. Maden. diophrbalmus Eur. $M r u f c a L$. apiformis Germ. vefpiformis Eur. $M u s c a ' L$. feftivus Eur. Mu/ca L. laetus Gerns. ruficoruis Dans. tricolor S. $N$. femirufus Gerns. calcaratus $A f r$. flavicornis Gerus. aeneus Germ. funefus Ital. melanch olicus Ital. fepulchralis Suec. $M u f c a L$.
Syrphys. triftis Germi, lugubris Dan. coemiteriorum Eur. $M u s c a L$. metallinus Germ. fubfultans Dan. glaucius Eur. Muf ca $L$. noctilucus Eur. $M u f c a L$. globofus Angl. Ribefii.

$$
\text { Mulea } L \text {. }
$$

2 fafciatus Eur.

Pyrafti Eur. $M u f c a$ L. Thymaftri Germ. transfuga Eur. $M u l c a L$. Salviae Sierr. Leon. Corollae Germ. umbellataruin $D a n$. abdominalis Gall. Rofarum Dan. mellinus Eur. $M u s c a$ L. Menthafri Eur. $M u f c a L$. fcalaris Germ. feriptus. IMusca $L$. Ocymi Gernn. difpar $A m$. m: nectareus Dan. albimanus Angl. dimidiatus Am. n.

SYRPHVs. 
Syrphvs. pipiens Eur. ") Mvsca. vomitoria $L$.

$M u f c a L$.

gibbus Barb.

gibbofus Ezr. Mufca L.

124. orbiculus Germ.

Mvsca. (Fliege. La Mou. che. $F(y$.)

* Antennis Seta plamata. jejuna Trancib. meridiana L. Eur. carnaria L. Eur. Am. ") carnivora Germ. lardaria Germ. ruficornis In:. Or. rutilans $A m$. $m$. maculata L. Eutr. rudis Germ. obfcura Germ. domeftica $L$. Eur. occidua Am. m. In . ftriata Dan. americana. retula $N$. Holl. putrida $A m$. $\ln$. Caefar L. Eur. megacephala Guim. felina Caft. $N$. cornicena Ital. cadaverina L. Eur. fygia $[\mathrm{H} f . N$. Terr. mortuorum L. Eur. leonina N. Hall. Ces.

q) La Mouche à grofles cuiuifSclmeisfiege.
Eur. ${ }^{\text {s) }}$ vefpillo Germ. cyanea $C . B . S$. macellaria Am. Inf. vulpina Eur. nigripes Gerin. corvina $G$ erm. teftacea Germ. pallida Dan. difcolor Ind. Or. nebulo Ind. Or. mactans Cajen. augur $N$. Holl. canina Angl. Gerss. Arigofa Germ. plebeja Grm. ruftica Dan. tigrina Angl. meditabunda Ital. elata Eur. lupuliua Seland. ludifica Germus. longipennis Germ. funcbris Dan. * * Antenuis feta muda. fera Eur. ') 4 punulata Germ. teflellata Germ. lurida Germ. 2 fafciata $A m$. Inf. groffa $L$. Etr. $N_{4}$ Musch.

8) La Mouche blevi de la viande.

t) La Mouche noir, à veri. tre jaune, noir dans le inilisu. 
Mvsca. hiftrix Ams. trenula L. Eavr. rotundata L. Enr. ") puparum Germ. larvatum $L$. radicum $L$. pagana $D a n$. varia Ind. Or: braficaria. ") coinurella Hifp. crafticornis Anv. Inf. Erinaceus $D_{n n \text {. }}$ vol vulus Itál. lateralis Germ. albifrons L. Dan. canicularis L. Eur. pluvialis L. Eur. fubfultans $L$. Eur. chorea Germ. labiata Dain. verna Gall. roralis L. Eur. feneftralis $L$. Eur. rugofa Germ. fenilis Germ. ferrata $L$. Eurr. nigricornis $\mathbf{l} n d$. $O r$. cinerea Ind. Or. fealaris Dan. 4 maculata Germ. cellaris $L$. cylindricornis Gall. ciliata Germin.

u) La Mòıche noir à ven. tre hemifpherique roux ta. sheté de noir.

v) La Mouche cylindriqus,
Mvsca. meteorica $L$. Eur. atrata Germu, Frit $L$.

Leprae L. Aiv. putris Eur. aterrima Gall. manicata Gall. Arigula Gall. cupraria $L$. Eur. w) surata Germ. aenea $I n d$. Or. polita $L$. Eur. viduata $L$. Eur. pubera L. Eur. fafciata $A m$. Inf. petronella L. Eur. cylindrica Germ. filiformis Germ. tipularia Barb. comprefta Cajen. ephippium Gall. elongata Sierr. Leor. longipes $C a j m$. annulata Cajert. criftata Barb. corrigiolata L. Dan. curfitans Angl. cimicoides Norw. pallipes Gcrm. ungulata L. Eur. ${ }^{x}$ ) nobilitata L. Eutur.

Mveca.

w) La Mouche doréc.

x) La Mouche dorce à trois nervures fur les ailes. Nemotele bronzée. 
Mvsca. equeftris Braf: notata Angl. glabrata Gall. nigripes Gall. arrogans L. Eur. longicornis Alm. InI. roftrata Dan. 4 fafciata Germ. diaphana $\mathrm{M}$. ferruginea Ind. Or. hiftrionica Ind. Or. fuilla Germ. flaveola Dom. furcata Gall. aratoria Barb. merdaria Germ. lutaria Germs. livens Dan. cucularia L. Eur. feybalaria $L$. Eur. Percoraria L. Eur. marginata Germ. fimetaria $\dot{L}$. Eur. nigripenuis Gall. . rufifrons Ital. graminum Gall. cincta Dane. reticulata Ital. contigua Braf. junctata Germ. umbraculata Gall. pennines $A m$ bor. lugens Sierr. Leon. moerens Gsin. Oleae Ital. clavata Germ.
Mvsc A. parietina $L$. Eur.

difcoidea Dass. connexa Gall. umbrarum $L$. Ezer. vibrans L. Eur. punctatum Gall. cynipfea $\boldsymbol{L}$. Eur. groffificationis L. Eur. Artemifiae Dan. combinata $L$. Esur. florum Ital. erythroceph. Suce. Arnicae L. Eur. pulchella Ital. Atylata Angl. ruficauda Gall. arcuata Germu. unbellatarum Angl. $M$. Hyofcyani $L$. Heraclei Germ. dorfalis Bohem. feminationis Germ. picta $A m$. b. vittata Guin. flava L. Eur. ') 4 punctata L. Eur. aeftuans $L$. Eur. lineata. Rofae Germ. Sertatulae L. Estr. germinationis $L$. Vrticae $L$. craflipenuis Germ. N5 Mvsca.

y) La mouche jaune aux yeux noirs. 
Mrsca. cornuta Germ.

Dauci Suec.

Cerafi $L$.

Ânnonae $A m$. $I n$. crux Ind. Or.

Cardui $L$.

Syngenefiae Germ.

Tutilaginis Dan.

fucata $A m s . m$. In $\int$.

folnitialis $L .{ }^{z}$ )

coltalis Am. m.! Inf.

Lychnidis Germ.

Onopordinis Dan.

Centaureac Germ.

Scabiofae Gall.

Hieracii Angl.

203. minuta Germ. ")

TABANvs. (Vibhbrime.

Le Taon.)

roftratus L. C. B. S.

haullellatus $H i f p$.

$T$. wauritanus $L$. probolcideus Barb. bovinus L. Etrr. b)

lineatus $A m$.

Morio Rarb.

z) Die Klitunfiege. Monche de la Bardane. La Mouche des têtes des Chardons,

a) Porro:

I) M. crythrophthalua. P.

2) - cynophila, P.

3) -. femorata. P.

4) - Rellata P. La c) Die Dorfbreme. LeTa. Mouche à etoile: "ougris à jambes fauves.

b) Le Tann à ventre jauna. tre et taches triainguaires blanshes.
T A B A N 8 . autumualis L. Eur.

fafciatus Sierr. Leon. calens L. Am. M. tarandinus L. Eur. albipes Gall. fervens $L$. $A \mathrm{~m}$. w. exaefluans L.. Am. wh. ruficornis $A m$. pellucens Germ. atratus $A m$. $I n j$. bromius L. Eur. cinctus $V$ irg. cajennenfis. nigrita Goll. italicus. nexicanus L. Surin. rufticus L. Eur. ') paganus Angl. punctatus Cajen. inanis Cajen. occideutalis L. Am. m. tropicus $L$. Eur. graecus 1tal. borealis Norw. antarclicus L. Am. m. pluvialis L. Eur. ") lineola Am. $b$. lumatus $B a r b$. equorum Germ. macularis Marocio.

TABA-

d) Die Regenbremse. Le'Ta. on à ailes bruiles piquées de blane. 
TABAN Y $S$ vittatus $M a$ -

\section{rocto.}

longicornis Braf.

Ariatus Chin.

2 dentatus Germ.

2 fpinofus Germ. caecutiens L. Eur. '

moerens Cajen.

feneftratus Gall.

coftatus.

lugubris L. Eur.

viduatus Germ.

48. fepulcralis Germ.

Rhingia.

roftrata Eur.

Conops $L$.

lineata Dan.

mufcaria Germ.

Asıuvs. (Raubfliege.

$$
\text { L'Afile.) }
$$

groflus $A m$. maurus $L$. Afr.

algirus $L$.

analis Ital.

barbarus L. Afr.

crabroniform. L. Eur. ephippium L. Germ. gibbofus Eusr. paedeinontan.

maroccanus. sureus Gall. barbatus Cajen. fafciatus Siery. Leon.

e) Le Taon brun, à cotes lu ventre jaune et ailes tachstées de noir.
Asicvs. aefluans $L$. An. $b$. ater L. Eur. Diadema ltal. calidus Cajen. flavus $L$. Etrs. atratus Barb. ruficornis Ital. violaceus Germ. gilvus L. Eur. punctatus Ital. cylindricus Germ. marginatus L. Eur. limbatus Barb. plumbeus $N$. Holl. brunneus Cajen. forcipatus L. Eur. $N$. Holl.

tibialis Germ. germanicus $L$. rufipes Ais. . maculatus Ind. Or. chinenfis. bruunipes Barb. narginellus $A m$. Inf. sunulatus Ind. Or. fylatus Am. Inf. nigripes $\mathrm{Cajen.}$ fabaudus Ital. cingulatus Ital. tjpuloides $L$. Eur. teutonus $L$. nitidulus 1 tal. cajennenfis. glaucius Ital. lineatus $A m$. 1n:. 
A SIL v s, auratus Ind. Or.

Ariatus Barb. arcuatus Ital. cinctus Germ. cyaneus $C . B . S$. oelandicus. L. Eur. hyalipennis Dan. frontalis Giemm. Morio Truis. hottentotta Germ. haemorrhoid. Barb. conopfoides N. Holl. linearis Dant. Inf. culiciformis Angl. mufcarius Gall.

63. minutus Ital.

Conops. (Stechfliege. Le

Stomexe.)

veficularis $L$. Estr.

aculeata $L$. Esur.

picla $A m$. $I n f$.

vittata Germ.

rufipes Germ.

erythroceph. Ind. Or. ')

macrocephala L. Eur.

8. flavipes L. Eur.

STOMOXYs.

Morio Braf. grifea Germ.

parafita $A m$.

fiberita Dan.

calcitrans.

Conops $L$.

f) L'Afile panaché de fau. re et de noir à ailes noires.
S т м M x s. teffellata

Germ. irritans L. Eur.

Conops $L$.

mufcaria Dan.

pungens Ezr.

afiliformis Ital.

Aylata Barb.

12. dorfalis Gail.

Myо га. (Blajenflie.

$$
\text { ge.) }
$$

dorfalis Germ. ferruginea $E u r$.

Conops $L$.

atra $D_{a n k .}$ teftacea Germ.

Conops $L$.

buccata Eur.

Conops $L$.

punctatil Germ.

cincta Ind. Or.

cingulata.

cinerea Ital.

I0. annulata Ital.

Cvlex. (Miikke. Le Cou-

(in. Tise Gnat.)

pipiens L. Eur. ansulatus $D$ an.

trifurcatus $L$. Esur.

lutefcens $D_{a n t}$

haemorrhoid. Cajen.

ciliatus Carol.

pulicaris L. Eur.

Am.

reptans $L$. Eur. b.

Morio Angl. 
Crlex. ro. equiaus $L$. B о м́хуIVs. minor $L$.

Eur.

EmpIs: (Schnepfenfliege.La

Mouche becafe.)

borealis L. Eur.

cylindrica Barb.

marginata Germ. clavipes Seland. penuipes L. Eur. forcipata L. Eusr. inaura Germ. livida L. Eur. ciliata Germ. teffellata Barb. Morio. cinerea Eur. nigripes Danz. fulcipes Dan. maculata Ital. ferciorea L. Euss. plumbea Ital. minuta Dar. flavipes Darr. nigripennis Dan.

I. melanoceph. Dar.

Bo mbYtrvs, (Schuebfiege. L'Afile velu. BนสวFly.)

major L. Eur. aequalis $A m$. $b$. medius L. Eur. planicornis $\mathrm{S}$. $N$. punctatus Barb. analis C. $B . S$. difcoideus $B a r b$. capenfis $\boldsymbol{L}$. nitidulus Germ.
Eur.

mininus Germ. cupreus Cajeq. ater $L$. Germ. maculatus Tranqb. melanoceph. Barb. fulcus Ital. grifeus Hifp. fenilis Am. m. virefcens $H i j p$. 20. pygmaeus $\{A m .6$. VOL VCCELLA. florea Barb. aurata $B a r b$. verficolor Barb.

Cytherea. obfcura Barb. aurea Barb.

HIP Pово SCA. (Lausfiege."

L' Hippobosque.) equina L. Eisr. Am. ") avicularia $L$.

Hirundinis L. Eur. $^{\mathrm{h}}$ ) ovina $L$.

Pycnogonvm.

Balaenarum Oc. Eur: Phalangiumn $L$.

NYMPHON. groflipes Mar. Norw. Phalangiuń L. hirtum $O_{c}$. Norwo.

\section{Pedie}

g) La Mouche à chien. Th horfe leech.

h) La Mouche araignée. 
P.edicylus. (Lials. Le Pedicylys. Recurvirontrae

\section{Pous. L.ouff.)}

humanus $L$.

Pubis $L$.

ricinoides $L$.

Cameli $L$.

Cervi $L$.

Ovis $L$.

Bufali C. B.S.

Bovis $L$.

Vituli $L$.

Equi.L.

Afini $L$.

Vulturis Ind. Or.

Tinnunculi $L$.

Buteonis.

Strigis.

Corvi $L_{\text {. }}$

Cornicis.

infaufti $L$.

Picae $L$.

Orioli.

Cuculi.

Cỹgni $L$. Anferis $L$.

Mergi

Procellariae Braf.

Vagelli.

Diomedeae Braf:

Sternae $L$.

Plataleae $\dot{L}$.

Ardeac $L$.

Gruis $L$.

Ciconiae $L$.

Charadrii $L$.

Fulicae L.
L.

Haematopi $L_{\text {: }}$.

Pavonis $\boldsymbol{L}$.

Meleagtidis L.

Gallinae $L$.

Phaliani.

Caponis $L$.

Tetraonis $L$.

Lagnpi $L$.

Columbae $L$.

Emberizac.

Pari L.

Motacillae.

Hirundinis $L$.

Gryllotalpae.

52. Apis $L$.

Acarves. (Mille. La Ti-

que. Tick.)

Elephantinus L. Ind:

Ricinus $L$.

Reduvius $\boldsymbol{L}$.

pallipes Germ.

hifpanus.

fanguifugus.

reflexus Ital. aegyptius $L$. undatus $N$. Holl.

lipfienfis.

marginatus Gall.

Iguanae.

cajennenfis.

lineatus $A m$.

aureolatus $A$ in.

holfatus.

reticulatus Gall. 
Acarvs, indus $L$. americanus $L$. vibrans Cajent. craffipes $L$. Eur. Vefpertilionis $L$. pafferinus $L$. coleoptratus $L$. motatorius $L$. aphidioides $I$.. telarius $L$. Eur. Siro $L$. feabiei. lactis $L$. dyfenteriae $L$. geniculatus $L$. baccarum $L$. Zofterae Oc. Norw.
ACARVS. Mufcarum

$$
L \text {. }
$$

Batatas $L$.

Gymnopterorum $L$. coleoptratorum $L$.

Caraborum Germo.

Phalangii.

Phaetontis $O i$. auftr. rupefris L. Eur. longicornis L. Eus. littoralis L. Eur. Fucorum Oc. Norw. Fungorum $L$. Tremellac $L$. falicinus $L$. croceus $L$. 50. Seminulum. P. Eur. 
208

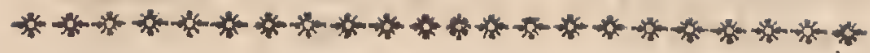

\section{A D.D E N D A.}

pag. 4r. Alburnus I. Alurnus.

pag. 78. Forficula Angl. Ear $\cdot$ wig.

Blatta Angl. Black-beetle, Cockroach.

pag. 80. Acheta domeftica Angl. the Cricket.

pag, 84. Podura Angl, Spring-tail.

pag. 85. Phryanea AngI. Caddice. Water-moth.

pag. 86. Termes, weifse Ameife, fourmi blanche, white ant, wood-ant, wood-loufe.

pag. 98. Chryfis Angl, Golden Fly.

pag. 99. Vespa. Angl. the Wafp.

- crabro Angl. the Hornet.

pag. 102. Apis terreftris. Die Hummel. the hwnubie bee. pag. 104. Formica Angl. Ant.

pag. I13. Phalangium Opilio Angl, the Shevherd. pag. 179, 1. adde Naucoris. 


\section{INDEX GENER VM.}

E. Eleuteratum. VI. Vlonatum. S. Synifatum.

P. Piezatum, O. Odonatum, M. Mitofatum.

Vn. Vuogatum Ag. Agonatum. G. Glofatuin.

R. Ryngotum. An. Antliatum.

\begin{tabular}{|c|c|c|c|}
\hline$A$ & & & \\
\hline Acanthia. R. & & $\begin{array}{l}\text { Bombylius. An. } \\
\text { Bombyx. G. }\end{array}$ & 205 \\
\hline Acarus, An. & 206 & Boftrichus. E. & \\
\hline Acheta. VI. & 80 & Brachycerus. E, & \\
\hline Acrydiuu, vl. & 79 & Brentus. E. & 72 \\
\hline Aeshna. $O$. & 108 & Bruchus E. & 4 \\
\hline Agrion, 0 . & 108 & Bupreftis. E. & $\begin{array}{l}54 \\
51\end{array}$ \\
\hline Alucita, $G$. & $17 \mathrm{I}$ & Byrrhuo. E. & 15 \\
\hline Alurıus, E. & $4 \mathrm{r}$ & C. & \\
\hline Andrena. P. & Ior & Callidium. E. & 0 \\
\hline Anobium. E. & 28 & Calopus. E. & 8 \\
\hline Authrax. An, & 194 & Cancer. Ag. & \\
\hline Anthrenus. E. & 30 & Cantharis. E. & 6 \\
\hline Anthribus. E. & 64 & Carabus. E. & \\
\hline Apalus. E. & $4 \pi$ & Caffida. E. & \\
\hline Apate. E. & 63 & Cebrio. E. & \\
\hline Aphis, K, & 188 & Cerambyx. E. & \\
\hline Apis. P. & 102 & Cercopis. R, & $\times 78$ \\
\hline Aranea. $V \mathrm{H}_{\text {. }}$ & $11 I$ & Ceria. An. & $x$ \\
\hline Afcalaphus. S. & 86 & Cerocouna. E. & 14 \\
\hline Afilus. An. & 203 & Cetonia, E. & \\
\hline Afacus. Ag. & 117 & Chalcis, $\mathbf{P}$. & \\
\hline Attelabus. E. & 60 & Chermes, R. & \\
\hline & & $\begin{array}{l}\text { Chryfis. P. } \\
\text { Chrofome F }\end{array}$ & \\
\hline $\begin{array}{l}\text { Bembex. } \\
\text { Bibio. An. }\end{array}$ & $\begin{array}{r}90 \\
194\end{array}$ & Cicada, R. & 34 \\
\hline Blaps. E.. & 17 & Cicindela, E. & \\
\hline Blatta. V], & 78 & Cimex. $\mathrm{R}$. & 180 \\
\hline
\end{tabular}


Ciftela. E. Cierus, E. Coccinella. E.

Coccts. R.

Colydium. E.

Conops. An.

Coretus- R.

Coffus. G.

Coffyphus. E.

Crabro. P.

Crioceris. E.

Cryptocephalus. E,

Cucujus. E.

Culcr. An.

Curculio. F.

Cymothoa. Ag.

Cynips. F.

Cytherea. An.

Cychrus. E.

Dermeftes E.

Diaperis. E.

Donacia. E.

Dorylus. P.

Dryops, E.

Dytícus, E,

Elater. E.

Elaphrus. E.

Elophorus. E.

Empis. An.

Ephemera, S.

Erodius. E.

Erotylus. E.

Evania. P.

Eucera. P.

Forficula. Vl.

Forinica. P.

Fulgora, R,

Galathea. Ag.

\section{$D$.}

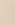

40 Gerris. R.

25 , Gryllus. VI.

31 Gyrinus. E. 25

189 H.

73 Helops. F.

204 Hemerobius. S.

183 Hepialıs. G.

153. Hefperia, G.

45 Heterocems. E. 30

101 Hexodon, E. 14

36 Hippa $\Lambda \mathrm{g}$. , 117

41 Hippobofca. An. 205

44 Hispa. E. 43

204 Hifter. E. * 14

66 Horia. E. 44

I19 Hyblaea. G. 159

89 Hydrophilus. E. 23

205 Hylacus. P. 10 I

22 Hypophlaetss. E. $\quad 73$

27 Ichnetunon. $\mathbf{P}$. 9 i

74 Ips. E. 74

46 Iulus, M. $\quad 109$

105 L.

24 Lamis. E. $\quad 56$

E. 24 Lampyris. E. 45

53 Larra. P. 97

23 Lepisma. S. 84

25 Leptura. E. 62

205 Lethrus. E. 10

84 Leucopfis. 13.99

16 Libellula. $0 . \quad 107$

39 Limmius. $\mathrm{Ag}$. $\quad 117$

95 Locufta. VI. $80^{\circ}$

104 Lucanus, E. 54

Lyctus. E. $\quad 73$

78 Lycus. E. 45

104 Lygaeus. $R$. . 183

I75 Lymexylon, E.

Lytta. E.

II $M$.

37 Manticora. E.

119 Mantis. Vl.
Galleruca, E.

Gaumarus. Ag.

\section{G.}


Malachius, E. Mafaris. P. Melafis. E. Mellinus P. Meloe E. Melolontha. E. Melyris, E。 Membracis, R. Miris. R.

Molorchus. E. Monoculus. Ag. Mordella. E. Murca. An, Mutilla l. Mydas. An. Mylabris. E. Myopa. An. Myrnieleon. S. Mycerophagus. E.

Naucoris, R. Necydalis. E. Nemotelus, Ar.

Nepa. R.

Nicrophorus, E.

Nitidula. E.

Noctua, G.

Nonrada. P.

Notonecta. R.

Notoxus, E.

Nymphon. An.

Oeftrus. An, Omalyfus. E. Onifeus. M.

Opatrum. E.

Oxyporus. $\mathrm{E}$.

Paederus. E.

Pagurus. Ag.

Panorpa. S.

Papilio. G.

Parnus. E.

Paft,lis. E.

N.

\section{0.}

$P$.

$P$.
27 Pediculus. An,

206

100 Phalaena. G.

29 Phalangium. Vn.

159

$100^{\prime}$ Philanthus. $R$.

74 Phryganea. S.

48 Pinelia. E.

27 Podura, $S$.

175 Prionus. E.

186 Pterophorus. G.

63 Ptilinus. E.

117 Ptinus. E.

46 Pulex. R.

199 Pycnogontm. An.

105 Pyralis. G.

194 Pyrochroa. E. 44

204 Ranatra. R.

86 Raphidia S.

73

179

62

196

I79

29

30

153

104

179

26

205

$$
193
$$

193 Scolopendra. M.

45 Scolytus. E.

109 Scorpio. Vn.

16 Scyllarus. Ag.

76 Semblis. $S$

Sepidium. E.

76 Sefia. G.

115 Sigara. R.

86 Silpha E.

127 Sirex. P.

29 Sphaeridiun,E.

55 Sphex. $P$.
113

100

85

16

84

55

$17^{2}$

29

28

187

205

166

45

179

86

187

196

59

203

66

46

41

59

74

10

16

I6

97

109

23

113

II 7

84

147

179

29

90

15

.95 
Index Genersm.

Sphinx, G.

Spondylis. E.

Squilla. Ag.

Staphylinus. E.

Stenocorus, E.

Stomioxis. An.

Stratiomys. An.

Synodendron. E.

Syrphus, An.

$T$.

Tabianus. An.

Tarantula Vn.

Tenebrio. E.

Tenthredo. P.

Termes. S.

Tettigonia. $R_{\text {, }}$

Thrips, R.

Tetratoma. E.

Thynnus, P.

145 Tillus. E.

63 Tinea. G.

119 Tiphia. P.

74 Tipula. An.

58 Trichius. E.

204 Tritoma. E.

195 Trox. E.

63 'Tragofita, E.

Ig6 Trombidium. Vn. Truxalio. VI.

202

II 3 Vespa. P.

17 Voluccella, An.

$89 \quad U$.

86 Upis. E.

$176 \quad Z$.

190 Zonitis. E.

74 Zуваела. G.

43

169

97

193

II I

79

98 Zygia. E. 


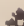

\section{INDEX NOMINVM GERMANICORVM.}

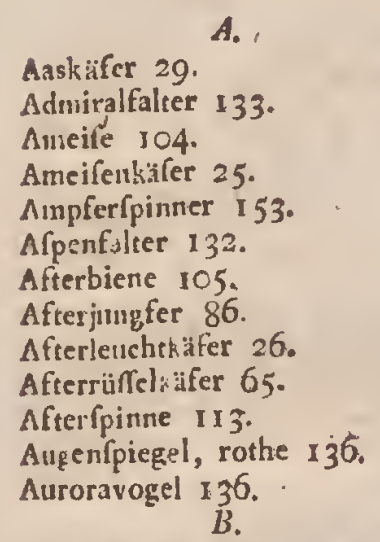

Bär, fchwarzer 152 .

Bärphalene I5t.

Baumuager 63.

Baunringelmotte 150 .

Baumweifsling 136 .

Beinwellfpinner 152.

Bettwanze 179.

Biene 102.

Bienenwolf 26 .

Birkenfpanmer 161 .

Blanfehiller I 4 I.

Blafentliege 204.

Blafenfuifs I 90.

Blattion 189 .

Blatılans 188.

Bhatifauger 189.

Blatt, das waindelnde 79 .

Blat wefpe 89.

Bockkifer 55 .

Bohrkäfer 28.

Bonbardierkäfer 20.

Borkenkäfer 63 .

Brandljäfer 45 .
Bremfe 193.

Bretfpiel 139.

Bronibeerfpanner 162 .

Buchenwickler 166.

Buntflüget 149.

Bürftenbock 56 .

Bürftenkäfer 64 .

Butterblumenfpinner 150.

c.

Cicade, blutrothgefleckte 178 . Weidenfchaum:78.

Citronenfchunetterling 136 . D.

Decklkäfer 22.

Dickkäfer 66.

Diftelfalter 132 .

Doppelband 178 .

Durfbreme 202.

Dotterweidenfpinuer 156 .

Drahtkäfer 73.

Dungkäfer 10.

\section{E.}

Eichenbufchfpinner 150 .

Einfchnittfiege 596.

Eisvogel 132.

Erdeichelfchwärmer $147^{\circ}$

Erdfloh 38.

Erdftaubkäfer 16.

Erdwühler II. F.

Fadenkäfer 40.

Fahne, fpanifche 151.152.

Fallkäfer 4I.

Falter, zackichter blankblaner

\section{1.}

Fanghenfelureclie 79 .

Federkanmmkäfer 29.

Federimotten 172 .

O 3

Feder- 
Federträger 29.

Fenterfalter 142 .

Feuerkäfer 45 .

Fichenzerführer 64.

Fliege 199.

Fliegenkäfer $44,62$.

Floh 188.

Flahkrehs 119.

Florfitege 85.

Flurskrebs 117.

Flıfsp:lanzenkäfer 46 .

Forchekäfer 37.

Forftiäfer 55 .

Fuchs; der grofse I 33.

- derkleine 133.

Fugenkäfer 15 .

Fusfeliwanzthiergen 84.

Galuelpe 89.

G.

Garbenfpinuer 152.

Gerber 55 .

Glanzkäfer 30. 45.

Goldeute 157 .

Goldglanz 157.

Goldhähnchen 34 .

Goldl äfer 47.

Goldkliimpgen 160.

Goldwefpe 98 .

Grabkäler I1.

Grashüpfer 81.

Grofskopf 150.

Gürtelkäfer 4 t. $H$.

Habichtskrantfpinner 149 .

Hackenkäfer 74 .

Haft 84.

Hage nbuchenfpinner I 49.

Hausfran 152 .

Hansk âfer 28.

Hauswurzfalier 136 .

Heiterneffelfalter 133.

Herzkäfer 74

Hetsfichreckenkufer 29 .

Hö:kerkäfer 16 .
Holunderfpanner 158 .

Holzbock 58.

Holzbohrer 44.

Holzlaus 56 .

Holzwurn 63.

Hopfenzïnpfler 165 .

Hornife 99.

Hummer 117 .

1.

Iuno 75 .

$k$.

Kaifervägtlein $15 \mathrm{I}$.

Kalmeichenfchwärmer 147.

Kancelfiege 86.

Kammkäfer 46 .

Kelleraftel 109.

Keulhorn 179.

Kiefenfurs 117 .

Kielkäfer 73.

Kleidermotte 170.

Klcttenfliege 202.

Kneipläfer 18.

Knollkäter 30.

Küniginnenpage 128 .

Kahlweifsling, der grofse 136.

Kolbenkäfer 10.

Kornwurm, der weifse r 72.

Krelss 115 . der fchwarze 67.

- moluckifche 117.

Kresiz, das grofse 22.

- das kleine 22.

Kronenkafer 44.

Kugelkäfer 15.

$$
4 .
$$

Laternträger 175 .

Lanfı̈̈fer 19.

Laus 206.

Eausfliege 205.

Lenchrkäfer 45.

Löucnzahnfpinner 149.

$M$.

Marienkafer $3 \mathrm{I}$. 
Marmorargus 139.

Manlwurfskiufer 30.

Mayerin I 52.

Maykiufer 48.

Maywurmkäfer 74.

Mehlkäfer 17 .

Mehlipanner 164 .

Milbe 206.

Molirenfliege 193.

Moosdifteleule 158 .

Morphens 144:

Motien 169 .

Miikke 204.

- Murfeikäfer 64.

$$
\text { N. }
$$

Nachifalter, fchwefelgelb. I 51.

Nachtkerzen fehwärmer I45.

Nagelfeck 149.

Nashornkäfer II.

Nierenfleck 141 .

0.

Oleanderfchwärmer 145 .

$$
P \text {. }
$$

Pappelweidenfpinner I 52.

Pelzmotte 170.

Perlnutterfalter 134.

Pfouentagfalter $\mathbf{I} \mathbf{3} \mathbf{I}$.

Pfiffèrkäfer 73 .

Pilzkäfer 74.

Pochkäfer 73 .

Polirfliigel 164 .

Prachekafer 51 .

Purpurhals 153.

\section{2.}

Queckenfalier 131 .

Quittenvogel i 49.<smiles>[R1]</smiles>

Rainweidenfchwärmer 146 .

Ranbfliege 203.

Raubkäfer 74 .

Rauchkäfer 43.

Regenbreme 202.

Reizkäfer 44.

Rindenküfer 44.
Rindennager 74 .

Ringbiene IOI.

Ritterlauf $n$ äfer 22.

Ritterfporucule 157 .

Rofenfpinner 150.

Rofsfliigel 153.

Rorhafter 11 .

Rothbuchenfpinner 149.

Rotheichenenle 155 .

Rothfufs 181 .

liothinals 153 .

Rübenwweif́ling 136 .

Ruffel säfer 66.

Sanderäber $x 6$.

Sandküfer 22.

Sandwefpe 95 .

Saumphaläne 156.

Schalie 78 .

Schatten':zfer 18.

Scheckthigel 133.

Schenkelwefpe 98.

Schiffwerftküfor 44.

Schild.äfer 33 .

Schildkrötenkăfer 26.

Schildlans 189 .

Sehillerfalter 132.

Schirmblumenkïfer 46.

Schleenfpinuer 150 .

Schlüffelblumeneule $\mathbf{5 6}$.

Schlupfküfer $I 6$.

Schlupfwefpe 91.

Schnislbock 62.

Schmalkïfer 43 .

Schineisfliege 199.

Schmetrerling 127 .

Selinacke 193.

Schneckenkiifer 80.

Silincelieufchrecke 80

Schnepfentlirge 196. 205.

Sehnurrkifer 35.

Schröter 54 .

Schuppenthierchen 84.

Schivalbenfchwanz 128 .

04
Schwanz- 
Schwanzwefpe go.

Schwebfliege 205.

Schwimnk fer 23.

Scolopender rog.

Scorpion 113.

Scorpiontiege 86.

See. Garncele II7.

Seegelfalter 128 .

Seenelken ́clıwrämer 148 .

Seidenküfer 40.

Sichelkleefehwïrmer 147.

Silbetifrieh 134 .

Eingheufehrecke 176 .

Speekiaifer 27.

Spindelbamnuotte $16 g$.

Spinne. II I.

Spinner, rolenrothe 153.

Spitzwegerichsfpinner $15 \mathrm{I}$.

Splintkäfer 63.

Springküfer 53 .

Stachelbeefpinner 162 .

Stachelkäfer. 43.46.

Stacheluacken 13.

Stammmotte 150.

Stamnringelvogel I 50 .

Stanbkäfer 74

Stechapfelfchwärner I 46 .

Stechfliege 204.

Steinkäfer 43.

Strandkafer 23.

Stumpihüfer 76 .

Stutzkäfer 14 .

Sumplkäfer 23.

Sumpffchwingel $157^{\circ}$

Tageflicge 84.

Tagvogel 127.

Tannenbock 56 .

Tannenueurm 64 .

Tafchenkrebs II 5 .

Tauhenhals I 48 .

'Tauchkürer 25.

Tieger 162.

Todtengräbeŕ 29 .
Todtenkäfer 17.

Todtenkopf 146.

Todtenprophet 1.T.

Traubenkifer 76 .

Trainerfalter 151 .

Trauernantel 132.

Trogel 149.

V.

Viehbrertie 202.

Vielfuls 109.

Violetfpitze 22.

Vulkan 133.

W.

Waffenfliege 195.

Walditrohf chwirmer 146.

Wanze, dickbeinichte 180 .

- Ichattirte 180.

- Bander 180.

- grasgrüne Baum - 182.

- Teich - 187.

- Fliegen-187.

Warzenkäfer 27.

Wafteringfer 107.

Wafterkäfer 24.

Waffermotte 85 .

Wafferrunzelkífer 25.

Wafferf́corpion 179.

Wafferwanze 179 .

Weisbuchenfpinner 150.

Wegerichsfpinner $15 \mathrm{I}$.

Wefpe 99.

Widderkäfer 60.

Windenmotte I $5 \mathrm{r}$.

Wittwe 152.

Wolfsmilch fchwärmer 146 .

Wurm, fchwarze 63 .

- Aiegende 63. $Z$.

Zalınbock 58.

Zahnwefpe 95.

Zangenboek 59.

Zangenkäfer 78 .

Zauberkäfer 56 .

Zünflèr $\mathbf{x} 66$. 


\section{NOMINA FRANCOGALLICA.}

A.

Abeille 102.

- des roches 103.

- à longues antennes 104. Altife bleu 38 .

- du Choux 38.

- noir nvale 38 .

- de la Mauve 39.

- fauve fans Aries 39 .

- - à ftries 39.

- janne 39 .

- à bandes janines 39.

Anthréne à broderie 30 .

Antribe noir ttrie 64 .

- uarbré 65.

Apollon 136.

Araignee 111 .

- fauteure II2.

Argentin 150.

Argus blen decoupé 141 .

- Catiné 142.

Arlequin doré 35 .

Arleguinć $6 \mathbf{r}$.

Arlequinette jutne I5I.

Arpentenfe de la ronce 162 .

Afile 203.

- panaché de funwe et de noir à ailes noires 204 .

- velu 205.

$B$.

Barlont II

Lecurare doré à êtuis rniges

65.

- vert 65 .

- doré 65.

- noir à pattes jaunes 66 . Bête de la Vierge 31.
Bibinn de St, Mare 194.

Biche petite 54 .

Binocle à queue en filets II7.

Blatte 78 .

Bowbardier 20.

Bout des bois 86 .

Bouclier grand noir 29.

- rabotellx 29.

- noir chifonne 29.

-..- folfoyeur 29.

Boufier à cornes retrouffées 13.

- à deux cornes 13.

- noir cormu 13.

- à pointes rouges 14.

Bruche fans ailes 29 .

Bronzé 142.

Buprefte tout noir 19.

- moir velouté rg.

- à cruis cuivereux 21.

- vert à bordure 21 .

- noir à corcelet rou. 'ge 21.

- vert à fix points blanches 23 .

- à tete canclée 23.

- à pointe $5 \mathrm{I}$.

C.

Cacrelat 78.

Calondre 67.

Cantharide 44.

- ardoife 26.

Capricorne Prione 55.

- odoriferant 55.

- Cavetier 56.

- noir unarbré de gris 56 .

à ctuis dentelées 56.

05

Capri. 
Capricorne noir chagrine 56 .

- charpentier 57.

- noir à aromes blan. cheatres $57^{\circ}$.

- à antenncs dentelées 59 .

- ponctire 59.

Carabé chagriné 19.

- petard 20.

- tête noir 21.

- Sycophante 21.

- à fix points $2 x$.

Caramote 117.

Cardinale 45 .

Caroline 108.

Caflide verte 33 .

- panachée 33.

Cerf volant le grand 54 .

Cetoine velis 48 . parallelipipede 54 .

Changeant 132 .

Chappe vert à bande 166 .

Charanfon cramoifi 65.

- _ du bonlean 65.

- du Trumble 65.

- de la velfe 66.

- noir violet 66 .

- tacheté des têtes de chordon 66.

- brnn du bled 67.

- de la Phellandrie 68.

- fauve 69.

- fatuear dir Saule 7o.

- gris ftric et fans ailes 70 .

- aे ctuis fauver 72.

Chaffeur des chenilles 19.

Chataigue noir 43.

Chevreste 117 .

- brune 18 .

Chevalier noir 22.

- rouge 22.
Chryfomele briquetée 35 .

- à rayes courbes 35 .

- bleue à bordure rouge 35 .

- noir à bordure ron. ge 35.

- à etuis bordécs 35 .

- fauteufe potagere 38.

- rée $39 . \quad$ vert do.

noir quarrée 42.

Cicindele tachciée 23.

- le rivage 23.

- i pattes jaunes 23.

- noir à deux points jaunes et corcelet noir 2 ?

- pelean 27.

- vert à points ronges 27

a bandes rouges 27 .

Cigale feuilletée à bande et ci. re 175 .

-176 .

- à bordure 177 .

- verte à tête panachée

177.

- jaune à raies nuires obli. ques 177 .

- à taches rouges 178 .

- bedcaude 178.

- à trois bandes blanches 178.

Cinips 89.

Ciftele pilule 15.

- ibande 15 .

- noir liffe 15.

- fatinée 15.

Citron 137.

Clairon fourni 25 .

- apivore 26.

- aे bandes rouges 26 .

- porte croix 26.

- blan 28. 
Cloporte Icg.

Coccinclle rouge aे 15 points noirs 32 .

- velue à taches rouges au corcelet 32 .

- rouge à 7 points noirs 31 .

Cochenille 189. mefteque 190.

Coquille d' or 172 .

Conlus 153.

Courtillere 80.

Coufin 204.

Crevette 117 .

- - des ruiffeaux IIg.

Criquet à Capuchon 79.

- enfanglante 82.

Criocere rouge du Lys 36 .

Cuculle 26 .

\section{D.}

Decoupure 156.

Demi paon 145 .

Denoifelle $10 \%$.

Dermefte bronfé 25.

- dulard 27.

- à deux points blancs

$$
27 .
$$

- à oreilles 29.

- à point d Hongrie 29.

- à deux points rouges 30.

Deuil demi 139 .

- Yetit 169 .

Diable le grand 175 .

Ditique large 24.

- à corcelet a baindes 24 . - filonnée 24.

Dos velu 48

Donue la belle 132.

Drap mortuaire 48 .

$$
E \text {. }
$$

Ecaille brune $15 \mathrm{l}$.

- marbrée 152.

- coulcar de rofe 152.
Ecaille marte 152.

Ecailleux violet 50 .

Ecreviffe 115.117.

Emeraudine 47 .

Ephemere 84.

Elcarbot noir 14.

- à taches rouges 15.

- d'ecaille 15.

Etoilée 158.

F.

Fancheur 113.

Flambé 128.

Forbicine 84.

Folloyeur 11 .

Foulon 29.

Fourmi 104.

Fourmillon 86.

Frelon 89. 101.

Frigane 85.

G.

Gamnua 133.

Gaze 136.

Gribouri bleu de l"Aune 37.

Grillon 80.

- des chanips 80.

Guepe 99.

- noir à cuiffes pofterieur fort grofles 95 .

- doréc 98.

$$
H \text {. }
$$

Hachette du Soiffunnois 149.

Hanneton 49.

- petit du Juin 49.

- à corcelet vert 50 .

- terrefte 10.

Heinerobe 85.

Hercule 10.

Hippobosque 205.

Homard 117.

Hydrophille le grand 23.

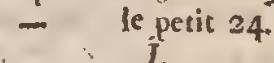

Jaspée 160. 
Ichneumon $9 r$.

Incarnat $15 \%$.

Ips typographe 63 .

Jule rog.

K.

Kermer 189.

- des Urangers 189.

- des racines Igo.

$L$.

Lepture aux croiflans dorés 61 . - à trois bandes dorés $6 \mathrm{r}$.

Licorne 67 .

Lion des Pucerons 85.

Livrée des arbres 150 .

I.on vermeon efpclis 189 .

Louife 108.

Lipere noir à corcelet et pat-

tes rnuges 37 .

- noir à pattes rouges 37.

\section{$M$.}

Mante 79.

Manteat bigarré 132.

Miroir I 44 .

Moine II.

Monocle II 7 .

- à coquille longue II

- à queue fourchue 117

Moro Sphinx 147.

Mordelle à tarriere 46.

Moucho Scorpion 86.

- ì frie 89.

- _- à tête rouge 90.

- Cigale r75.

- becalfe 205 .

- a chien 285 .

- araignce 205 .

- à ailes noires hordées de blanc onde 195 .

- arinée i ventre plat, chargée de fix lunules 195.
Mouche armée à corectet ronge fatiné 195 .

- 195. a ventre vert noires 195.

- _ noire à taches jaunes 195.

- - - à ventre et cuiffes jaunes 195 .

- à corcelet ftrie et ban. des jaunes interrompues fur le ventre 197.

- apiforme 197.

- imitante la guêpe d̀ longues antennes 197.

- dorée à ventre bruil et noir 197 .

- à fegment 197.

- à groftes cuifter 199 .

- grande 199.

- bleu de la viande 199.

- noir à ventre jaune, noir dans le miliei 199.

- - - - hemi. fphérique roux tacheté de noir 200.

- cylindrique 200.

- dorée 200.

- - d trnis nervires fir les ailes 200 .

- jaune anx jeux noirs 201.

- de la Bardane 202.

Mouchetée 162.

Mutilla 105.

N.

Naficorne Ir.

Naucnre 179.

Nenotele bronzéc 200.

Nueré grand 134.

0 .

Oeftre 193. 
P. Puceron 188 .

Pacifique 150.

Pailletre 39 .

Panache brune 29.

- janne 29 .

Paon du Jour 131 .

Papillon 127.

- page de la reine 128.

- du fenoiil 128.

- des Alpes 136.

- blanc du chou 136.

- blane veiné du vert 136.

- de la Canicule 137.

Paquet des feuilles feches 149.

Perce oreille 78 .

Perle brune à rajes jaunes 85 .

Phalangitte 10.

Phalene chinéc 152 .

- perite queue donhle 152.

- dorécàt taches argentées 157.

- blanche tachetée de noir $16 x$.

- à ventre relevé 164 .

- verd ondée 166.

Pillulaire grand 12 .

- petit 12.

Pinceurs 166.

Plein chant 145.

Plutus 39.

Podure 84.

Porte- lanterne 175.

Porte - quene 128.

- - à bandes fauves 141.

- bleu à une ban.

Pour 206.

Precoce 158.

Pregadion 79.

Prione 55:

Profcarabé 74.

P'terophores 172 ,

Puce 188.

Scolyte 64 .

Scorpion 113.
Punaife nayade $187^{\circ}$

- mouche 187.

- à avirons la grande 179.

- - la petite 179 .

- de lit 179.

- tigre 179.

- Siamoife 180.

- i pattes rouges $18 x$.

- verte 182.

- brune à antennes ct bords panachés 182 .

- rouge du choux 182 . :

- noire à quatre taches blanches 183 .

- verte à raies et taeher ronges on blanches 183 .

- verte blenatre 183.

- noir 183.

- - à tête allongée 183.

- d'eau 179.<smiles>[C+]1=CC1</smiles>

Queue fourchue 149. R.

Raphidie 86:

Richard 5I.

Robert le Diable 133.

Rofalie 56.

Rofette 153.

$S$.

Sablier 16.

Santerclle it contelas $8 \mathrm{r}$.

Scarabé 10.

- bedean x r.

- à nuque epineule I3.

- de cuir noir 46.

- onctueux 74.

Scarable à larges cuiffes $\mathbf{I} 6$.

Scolopendre 109.

- araignće 113.

- À corps uvale 179.

Scerpion 
Scorpion aquatique 179.

Silvain 132.

Sphex 95.

Sphinx du Tilleul 145.

- à tête de mort 146 .

- de Tithymale 146.

- du caille-lait 146.

- à cornes du boeuf 146 .

- du Troënne 146.

- Belier 147.

- de Piffenlit 547.

- de la Luzerne 147.

Staphylin bourdon 74 .

- grand woir liffe 75.

- petit noir 75 .

- bleu 75.

- à étuis conleur de rouille 75 .

- Junon 75 .

- jaune à tête étuis et ants noir 76 .

- couleur de paille 76 .

- de rivages 76 .

- noir à corcelet rouge 76.

Stenocore bedeau 62 .

Stomoxe 204.

Soufrée à queue I 59.

Souterrain 11.

\section{$T$.}

Tabac d'Efpagne I 34 .

Taon à ventre jatmatre et ta. ches triangulaires blanches 202.

- gris a jambes fauves 202

- à ailes brunes piqquées de blanc 202.

- brun à cotes du ventre jaune et ailes tachetées de noir 203.
Taupin ronge 53 .

- noir à corcelet rouge 54.

Teignes 160

Telephore à deux points jau. nes 27.

- verd bronze 27 .

rouges 27 .

- à bandes rouges 27.

Tenebrion d ftries dentelées 16.

\section{ment 17 .}

life a prolonge.

Tête armée II

Thrips 190.

Tique 206.

Tipule 193.

Tircis 131.

Toupet a pointes 165 .

Tourquet nageur 25.

Turquoife 148.

V.

Vache à Dieu $3 \mathrm{r}$,

Ver à Soye 150.

- luilant 45.

Verficolore 149.

Vert doré 157.

Vertu bleu petit 36 .

Vetive 153.

Volucelle à ventre blanc en de. vant 196.

Vonlcain 133 .

Vrilette carnaciere 29.

Vrucere 90.

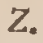

Zigzag. 150. 


\section{NOMINA ANGLICA.}

A.

Ant 208.

Argus blew 142 .

Bee IC2.

Beetle Io.

- Black 208.

- Hercules ra

- Musk 55.

- Oyl 74.

- Gogle 51.

Bug 180.

Bugg 10.

Butterfiy $\Sigma 27$.

- alpine 136 .

- Cabbage 136.

- white veined 136 .

- Brimftone 137.

Buzz-Fly 205.

c.

Caddice 208.

Cockroach 208.

Crab 115.

Crane - Fly 193.

Cravififh 117 .

Cricket $2 \mathrm{c} 8$.

D.

Iragun - Fly IOZ.

E.

Enfield Eye 13 J.

Ear - wig 208.
Flea 188 .

Fly 199.

- Buzz 2C5.

- Crane I93.

- Dragon 10\%.

- Gad 193.

- Golden 208.

- Lanthorn 175 .

- Stag - Flying 54.

- Todili io.

Fritillary 134.135 .

G.

Gad. Fly 193.

Glowworm 45.

Guat 204.

Golden. Fly 208.

Grashopper 81.

Greene Chafer 47.

H.

Hair ftreack brown 14 r.

- blew I4I.

Half Mourner 139.

Helined Firh 117 .

Hornet 208.

Horfe leech 205.

- Thoe 117.

Humble Bee 208.

L.

Lady - bird $3 \mathrm{I}$.

Lady - cow 3 I.

Lady painted 132. 
Lanthorn - Fly 175.

Lobfter II?

Loffan 67.

- Chatepeleafe 67.

Loufe 206.

$$
P \text {. }
$$

Peakocks Eye 13x.

Plautloule 188.

Punger 115.

s.

Sand - Crab II 5.

Shepherd 208.

Silkworm 150.

Silverconma 133 .
Spider III.

Spring-tail 208.

Stag Flying Fly 54.

$T$.

Tick 206.

Toddy-Fly 10.

Tortoifefhell 142.

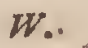

Wall loufe 179.

Walp 208.

Water inoth 208.

White ant 208.

Wood ant 208.

Wood loule 208. 


